\title{
Magnetic Priming of a Relativistic Magnetron
}

\author{
by
}

\section{Brad Winston Hoff}

A dissertation submitted in partial fulfillment of the requirements for the degree of Doctor of Philosophy (Nuclear Engineering and Radiological Sciences) in the University of Michigan

2009

Doctoral Committee:

Professor Ronald M. Gilgenbach, Chair

Professor Yue Y. Lau

Professor Eric Michielssen

Adjunct Associate Professor John W. Luginsland, NumerEx Senior Research Physicist Michael D. Haworth,

Air Force Research Laboratory 


\section{Brad Winston Hoff \\ (C) 2009 \\ All Rights Reserved}




\section{Acknowledgements}

This research was supported by the Air Force Office of Scientific Research, the Air Force Research Laboratory, L-3 Communications, Northrop Grumman Corporation, and the AFOSR MURI on Cathodes and Windows for HPM. I would also like to acknowledge the Directed Energy Professional Society and their support through the Directed Energy Professional Society Graduate Scholarship.

I would like to thank my research advisor, Professor Ronald Gilgenbach, as well as Professor Y. Y. Lau, Professor Eric Michielssen, Adjunct Associate Professor John Luginsland, and Dr. Michael Haworth for serving on my dissertation committee. Additionally, I would like to acknowledge the contributions made by Dr. Michael Haworth and Dr. Peter Mardahl of the Air Force Research Laboratory in collaborative research on microwave window breakdown phenomena presented in this dissertation. Finally, I would like to extend my sincerest gratitude to my family, friends, colleagues, and mentors who have supported me. 


\section{Table of Contents}

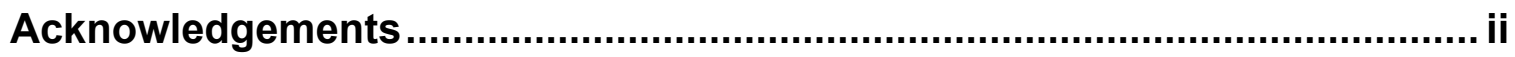

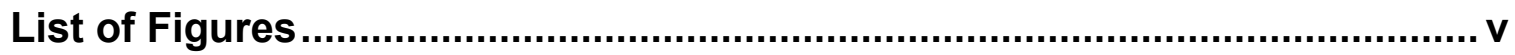

List of Appendices .....................................................................................

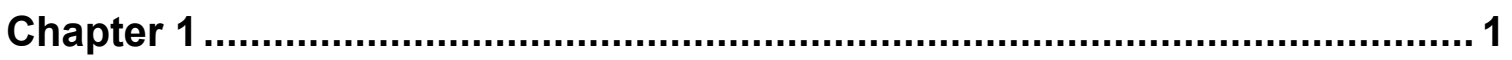

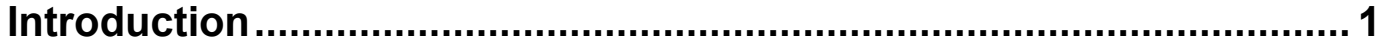

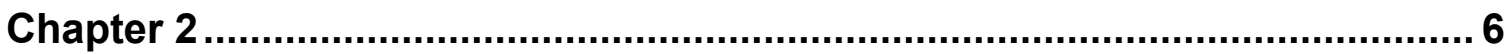

Basic Magnetron Theory and Background ......................................... 6

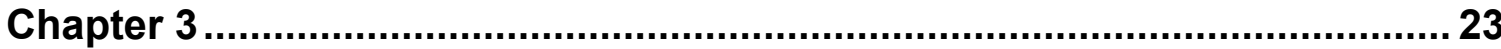

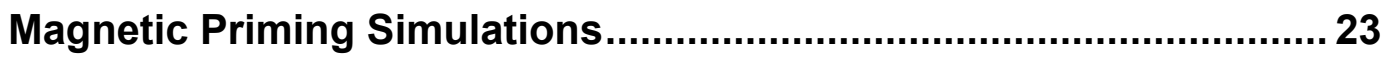

3.1 Magnetostatic Simulation Setup and Results................... 24

3.2 Electromagnetic Particle-in-Cell Simulations .................... 41

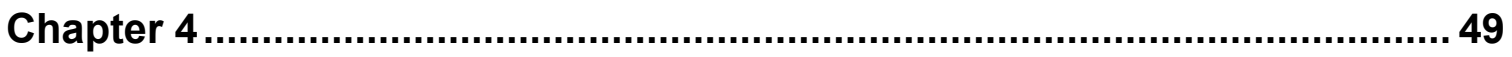

Magnetic Priming Experimental Research ....................................... 49

4.1 Experimental Configuration .............................................5 50

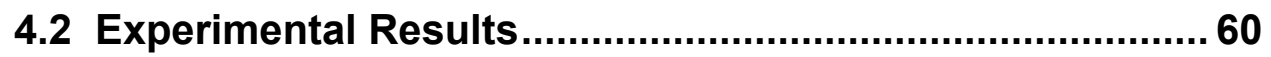

4.2.1 Magnetic Priming at the Cathode (Unbalanced

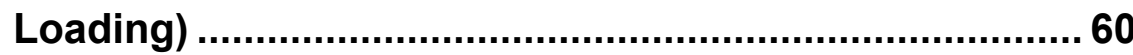

4.2.2 Magnetic Priming at the Cathode (Balanced

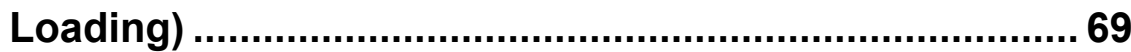

4.2.3 Magnetic Priming at the Anode ............................. 80

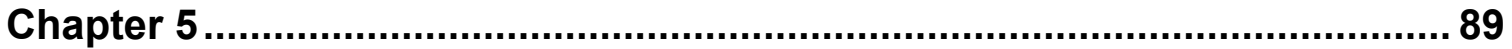

Magnetic Priming: Analysis, and Discussion.................................. 89

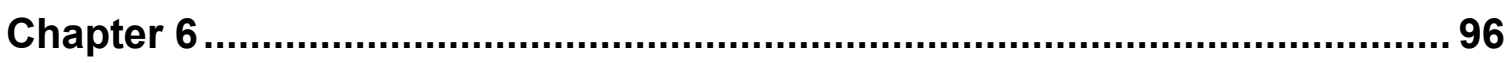

Dielectric Microwave Window Breakdown ......................................... 96

6.1 Experimental Configuration .................................................... 97

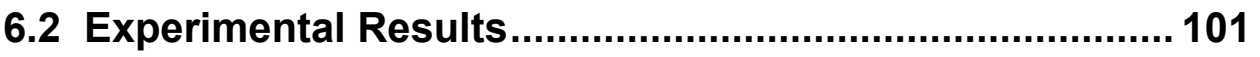


6.3 Simulation Parameters and Results .............................. 109

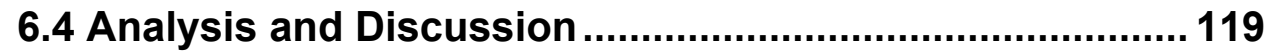

6.5 Implications on Magnetic Priming Results ..................... 123

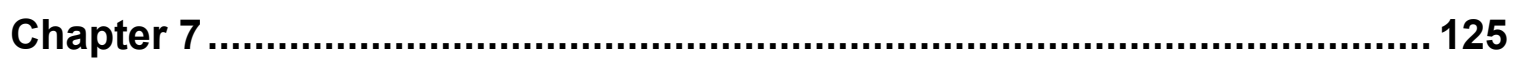

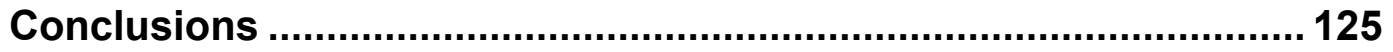

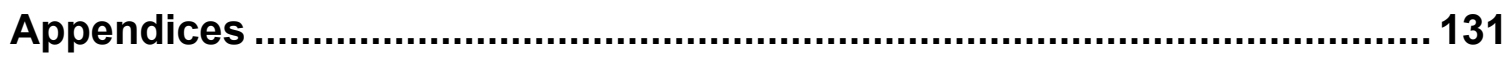

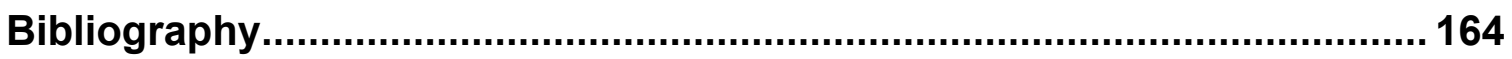




\section{List of Figures}

Figure 1. (a) Magnetically primed oven magnetron (after Neculaes [11]). (b) Computer model of a magnetically primed oven magnetron.

Figure 2. Computer model showing a portion of the UM/L-3 relativistic magnetron cathode with attached electrostatic end balls. (b) Close-up cutaway view of the cathode emission region showing wire locations.

Figure 3. Uncorrugated planar magnetron, showing an electron trajectory when the Hull condition is barely satisfied.

Figure 4. Uncorrugated planar magnetron with an applied magnetic field greater than required for Hull cutoff, showing multiple electron trajectories. 10

Figure 5. Uncorrugated planar magnetron with laminar (Brillouin) electron flow.

Figure 6. Corrugated planar magnetron showing RF wave and electron Brillouin flow.

Figure 7. Generic diagram of magnetron operating curves, showing the Hull cutoff and Buneman-Hartree resonance condition. Reproduced from Ref. [2]. . 14

Figure 8. Corrugated planar magnetron operating in the m-mode. Two electrons are shown, each in a different phase of the RF wave.

Figure 9. Corrugated planar magnetron. An electron is shown in phase with the wave. Positions $A$ and $B$ are shown as the reference positions.

Figure 10. Corrugated planar magnetron simulation. (a) Laminar flow prior to start of oscillation. (b) Spoke formation after the start of oscillation. Here, the external magnetic field points out of the plane of the paper.

Figure 11. A 6-cavity cylindrical magnetron representation, showing applied electric and magnetic field orientations as well as electron drift direction.

Figure 12. A 6-cavity cylindrical magnetron simulation. (a) Laminar Brillouin flow prior to start of oscillation. (b) Spoke formation after the start of oscillation....... 20 
Figure 13. Cut-away view of an oven magnetron, showing shorting rings (straps) and other internal structures.

Figure 14. (a) Magnetic field coils on the UM/L-3 relativistic magnetron experiment. (b) Magnetic field coil representation in the Field Precision Magnum magnetostatics software.

Figure 15. Contour plot of the axial magnetic field for the baseline case (no magnetic priming) A: Anode backwall (radius $=8.3 \mathrm{~cm}$ ), B: Anode block inner boundary (radius $=3.2 \mathrm{~cm}$ ), C: Cathode boundary (radius $=1.1 \mathrm{~cm}) \ldots \ldots \ldots \ldots . .27$

Figure 16. Axial magnetic field as a function of angle at radii $1.1 \mathrm{~cm}-3.1 \mathrm{~cm}$ (cathode radius $=1.1 \mathrm{~cm}$ ) for Baseline case (no magnetic priming).

Figure 17. Contour plot of the axial magnetic field for the case of magnetic priming at the cathode. Wire length $=4 \mathrm{~cm}$. Wire relative magnetic permeability $=1000$. A: Anode backwall (radius $=8.3 \mathrm{~cm}$ ), B: Anode block inner boundary $($ radius $=3.2 \mathrm{~cm}), \mathrm{C}:$ Cathode boundary $($ radius $=1.1 \mathrm{~cm}) \ldots \ldots \ldots \ldots \ldots \ldots \ldots \ldots \ldots \ldots \ldots \ldots \ldots \ldots \ldots . .28$

Figure 18. Axial magnetic field, $B_{z}$, as a function of angle at Radii $1.1 \mathrm{~cm}-3.1 \mathrm{~cm}$ (cathode radius $=1.1 \mathrm{~cm}$, wires $\mu_{\mathrm{r}}=1000$ ) for the case of magnetic priming at the cathode. Unprimed (baseline) case shown for comparison. 29

Figure 19. Contour plot of the axial magnetic field for the case of magnetic priming at the anode. Wire length $=4 \mathrm{~cm}$. Wire relative magnetic permeability $=$ $1000 \mathrm{~A}$ : Anode back wall (radius $=8.3 \mathrm{~cm}$ ), B: Anode block inner boundary (radius $=3.2 \mathrm{~cm})$, C: Cathode boundary (radius $=3.2 \mathrm{~cm}$ ), C: Cathode boundary $($ radius $=1.1 \mathrm{~cm})$.

Figure 20. Axial magnetic field, $B_{z}$, as a function of angle at radii $1.1 \mathrm{~cm}-3.1 \mathrm{~cm}$ (cathode radius $=1.1 \mathrm{~cm}$, wire $\mu_{\mathrm{r}}=1000$ ) for the case of magnetic priming at the anode. Unprimed (baseline) case shown for comparison.

Figure 21. Contour plot of the axial magnetic field for the case of combined magnetic priming at the cathode and the anode. Wire length $=4 \mathrm{~cm}$. Wire relative magnetic permeability $=1000$; Radial locations: $A$ : Anode backwall (radius $=8.3 \mathrm{~cm}$ ), B: Anode block inner boundary (radius $=3.2 \mathrm{~cm}), \mathrm{C}$ : Cathode boundary $($ radius $=1.1 \mathrm{~cm}$ ).

Figure 22. Axial Magnetic Field as a Function of Angle at Radii $1.1 \mathrm{~cm}-3.1 \mathrm{~cm}$ (Cathode Radius $=1.1 \mathrm{~cm}$, wire $\mu_{\mathrm{r}}=1000$ ) for the case of combined magnetic priming at the cathode and anode. Unprimed (baseline) case shown for comparison

Figure 23. Close-up cutaway view of the cathode emission region showing wire locations. 
Figure 24. (a) Contour map of $|\mathrm{B}|$, showing relation to the magnetic priming wires. (b) Contour map of $|\mathrm{B}|$ with $\mathrm{X}-\mathrm{Z}$ magnetic field vector overlay (priming wire shown in gray). The strong radial component of the $B$ field at the lower boundary of the cathode is due to the combined effects of the two neighboring wires outside the contour slice. 35

Figure 25. $|\mathrm{B}|$ contour plane references $(\mathrm{a}) \mathrm{Z}=0 \mathrm{~cm}(\mathrm{~b}) \mathrm{Z}=-2.6 \mathrm{~cm} \ldots \ldots \ldots \ldots . \ldots 36$

Figure 26. Contour map of $|B|$ with $X-Y$ magnetic field vector overlay (priming wire shown in gray), where (a) $Z=0 \mathrm{~cm}$ and (b) $Z=-2.6 \mathrm{~cm}$.

Figure 27. Magnetic field component plots for the $Z=0$ plane at various radii (a) $\mathrm{B}_{\mathrm{r}},(\mathrm{b}) \mathrm{B}_{\theta},(\mathrm{c}) \mathrm{B}_{\mathrm{Z}}$.

Figure 28. Magnetic field component plots for the $Z=-2.6 \mathrm{~cm}$ plane at various radii (a) $B_{r}$, (b) $B_{\theta}$, (c) $B_{z}$

Figure 29. MAGIC simulation geometry. (a) Radial cross-section of simulation magnetron. (b) Axial cross-section of simulation magnetron. (c) 3-D view of simulation magnetron (only cathode and anode vanes are visible, other components not shown).

Figure 30. Magnetron RF electric field amplitude as a function of time for the case of magnetic priming at the cathode. (Wire $\mu_{r}=1000$ ). The magnetic contour plot to the right of the graph is shown as a visual reference of imposed field perturbations.

Figure 31. Image from a MAGIC PIC simulation illustrating excessive electron endloss.

Figure 32. Magnetron RF electric field amplitude as a function of time for the case of magnetic priming at the cathode. (Wire $\mu_{\mathrm{r}}=500$ ). The magnetic contour plot to the right of the graph is shown as a visual reference of imposed field perturbations. A small degree of mode competition was observed in the $4 \mathrm{~cm}$ wire case, as evidenced by the beat pattern imposed on the power trace.

Figure 33. Magnetron RF electric field amplitude as a function of time for the case of magnetic priming at the anode. (Wire $m u=500$ ). The magnetic contour plot to the right of the graph is shown as a visual reference of imposed field perturbations.

Figure 34. Magnetron RF electric field amplitude as a function of time for the case of combined magnetic priming at the cathode and the anode. (Wire mu = 500 ). The magnetic contour plot to the right of the graph is shown as a visual reference of imposed field perturbations. 
Figure 35. Photograph of the UM/L-3 Titan relativistic magnetron, showing attached waveguides in the unbalanced loading configuration used in the first magnetic priming experiment.

Figure 36. Schematic diagram of the UM/L-3 Titan relativistic magnetron experiment used in the first magnetic priming experiment, illustrating approximate locations of the experiment diagnostics (reproduced from Ref. [31]). 52

Figure 37. Photograph of the UM/L-3 Titan relativistic magnetron, showing attached waveguides in the balanced loading configuration used in the second and third magnetic priming experiment.

Figure 38. Schematic diagram of the UM/L-3 Titan relativistic magnetron experiment used in the second and third magnetic priming experiment, illustrating approximate locations of the experiment diagnostics.

Figure 39. Illustration of the magnetic priming cathode, showing support rod geometry and magnetic wire locations.

Figure 40. Unmodified UM/L-3 relativistic magnetron anode block (a) photo and (b) dimensions.

Figure 41. Modified UM/L-3 relativistic magnetron anode block showing $1.27 \mathrm{~cm}$ diameter boreholes in each of the anode vanes. 58

Figure 42. $20.64 \mathrm{~cm}$ long, $1.27 \mathrm{~cm}$ diameter boron nitride rod (white) with 0.508 $\mathrm{cm}$ diameter magnetic priming wire mounted.

Figure 43. Experimental data points near the m-mode Buneman-Hartree resonance curve for the UM/L-3 Titan relativistic magnetron.

Figure 44. (a) Microwave power, incoming diode current, and diode voltage for a magnetically primed magnetron shot (shot 11065). The unlabled light blue trace in (a) is from a diagnostic not used in these experiments. (b) (upper) Magnetron heterodyne microwave signal (local oscillator set to $1.1 \mathrm{GHz}$ ) and (lower) corresponding TFA plot

Figure 45. (a) Microwave frequency at peak power as a function of applied magnetic field for the magnetically primed UM/L-3 Titan relativistic magnetron. (b) Microwave frequency at peak power as a function of applied magnetic field for the unprimed UM/L-3 Titan relativistic magnetron. All shots below the $\pi$-mode demarcation line are considered $2 \pi / 3$-mode shots 64

Figure 46. (a) Peak microwave power versus pulse width for all m-mode shots in the magnetically primed versus unprimed configurations. (b) Mean values of peak microwave power versus pulse width for the magnetically primed versus unprimed configurations. 
Figure 47. Mean values of magnetron m-mode oscillation starting time and time to peak power for the magnetically primed versus unprimed data sets.

Figure 48. Magnetron starting current for $\pi$-mode shots in the magnetically primed versus unprimed cases.

Figure 49. Experimental data points near the $2 \pi / 3$ and $\pi$-mode BunemanHartree resonance curves for the UM/L-3 Titan relativistic magnetron. (a) $4 \mathrm{~cm}$ cathode wires, (b) $8 \mathrm{~cm}$ cathode wires, (c) $12 \mathrm{~cm}$ cathode wires, (d) $16 \mathrm{~cm}$ cathode wires, (e) $20 \mathrm{~cm}$ cathode wires, (f) $27 \mathrm{~cm}$ cathode wires, (g) unprimed (no wires).

Figure 50. (a) Microwave power, incoming diode current, end loss current, and diode voltage for an unprimed bimodal magnetron shot (shot 12428). (b) (upper) Magnetron heterodyne microwave signal (local oscillator set to $1.1 \mathrm{GHz}$ ) and (lower) corresponding TFA plot ....

Figure 51. Shot percentages for the case of magnetic priming at the cathode with balanced loading.

Figure 52. Mean values of peak total microwave power versus pulse width for the baseline and cathode wire cases for (a) $\pi$-mode shots and (b) $2 \pi / 3$-mode shots.

Figure 53. Mean values of magnetron m-mode oscillation starting time (a) and time to peak power (b) for the magnetically primed versus unprimed data sets. 78

Figure 54. Magnetron starting current for $\pi$-mode shots in the magnetically primed versus unprimed cases

Figure 55. Magnetron energy efficiency for $\pi$-mode shots in the magnetically primed versus unprimed cases.

Figure 56. Experimental data points near the $2 \pi / 3$ and $\pi$-mode BunemanHartree resonance curves for the UM/L-3 Titan relativistic magnetron. (a) $10 \mathrm{~cm}$ anode wires, (b) $15 \mathrm{~cm}$ anode wires, (c) $20 \mathrm{~cm}$ anode wires, (d) unprimed (no wires)

Figure 57. Shot percentages for the case of magnetic priming at the anode with balanced loading.

Figure 58. Mean values of peak total microwave power versus pulse width for the baseline and anode wire cases for (a) $\pi$-mode shots and (b) $2 \pi / 3$-mode shots. 84

Figure 59. Mean values of magnetron $\pi$-mode oscillation starting time (a) and time to peak power (b) for the magnetically primed versus unprimed data sets. 86 
Figure 60. Magnetron starting current for $\pi$-mode shots in the magnetically primed versus unprimed cases.

Figure 61. Magnetron energy efficiency for $\pi$-mode shots in the magnetically primed versus unprimed cases.

Figure 62. Schematic diagram of the UM/L-3 Titan relativistic magnetron experiment in the original configuration. (a) Approximate locations of the experiment diagnostics (reproduced from Ref. [31]). (b) Dielectric window positioning.

Figure 63. Lucite window used in the original microwave window configuration. Window dimensions are $22 \mathrm{~cm} \times 14 \mathrm{~cm} \times 1.27 \mathrm{~cm}$. 99

Figure 64. Schematic diagram of the UM/L-3 Titan relativistic magnetron experiment in the new upgraded configuration. (a) Approximate locations of the experiment diagnostics. (b) Dielectric window positioning. 100

Figure 65. Uninstalled Upgraded Microwave Window 101

Figure 66. Relativistic magnetron performance in the original configuration (magnetron shot 12251). (a) Accelerator voltage, entrance current, and single waveguide microwave power. (b) Heterodyned signal and time frequency analysis of the microwave signal.

Figure 67. Single waveguide microwave power and arc detector output for magnetron in original window configuration (shot 12251).....

Figure 68. Photograph of the damaged microwave window from the original configuration, looking toward the coupling slot and the center of the magnetron.

Figure 69. Relativistic magnetron performance in the new, upgraded configuration (magnetron shot 12294). (a) Accelerator voltage, entrance current, end loss current, and single waveguide microwave power, (b) Heterodyned signal and time frequency analysis of the microwave signal for magnetron. ...105

Figure 70. Single waveguide microwave power and arc detector output for magnetron in the new configuration (shot 12294). 106

Figure 71. Microwave power and pulse length for original versus new upgraded window configurations. 107

Figure 72. Simulation model geometry. (a) XY Plane Cross Section (units in $\mathrm{cm}$ ). (b) YZ Plane Cross Section (units in $\mathrm{cm}$ ) [ICEPIC data courtesy of P. J. Mardahl]. 
Figure 73. Input voltage, anode current, and average single waveguide microwave power for the magnetron simulation (aperture emission enabled) [ICEPIC data courtesy of P. J. Mardahl]

Figure 74. Cross sections of the operating simulation magnetron at $t=199.343$ ns, showing the location of the electrons emitted from the microwave aperture. (a) X-Y plane cross section (cathode electrons are given a darker color for contrast). (b) Y-Z plane cross section [ICEPIC data courtesy of P. J. Mardahl].

Figure 75. XY plane cross section of the simulation, showing the cathode electrons (dark blue) and the aperture electrons (light green) over one RF cycle: (a) $198.999 \mathrm{~ns}$, (b) $199.293 \mathrm{~ns}$, (c) $199.539 \mathrm{~ns}$, and (d) $199.882 \mathrm{~ns}$ [ICEPIC data courtesy of P. J. Mardahl].

Figure 76. Accumulated charge integrated over the surface of the microwave window [ICEPIC data courtesy of P. J. Mardahl].

Figure 77. Particle impact locations on the front face of the microwave window [ICEPIC data courtesy of P. J. Mardahl].

Figure 78. Distribution functions for (a) impact energy and (b) impact angle for all particle impacts on the microwave window [ICEPIC data courtesy of P. J.

Mardahl].

Figure 79. Particle impact energy plotted as a function of impact angle for all particles striking the microwave window [ICEPIC data courtesy of P. J. Mardahl].

Figure 80. Distribution of predicted secondary emission yields for all simulation particle impacts [ICEPIC data courtesy of P. J. Mardahl].

Figure 81. Individual waveguide power traces for MELBA-C shot 12435 showing variations in measured power among the waveguides. 


\section{List of Appendices}

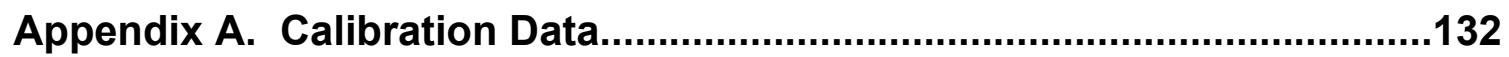

Appendix B. Equipment Technical Drawings and Pictures........................139

Appendix C. Additional Magnetic Priming Data..........................................152

Appendix D. Experimental Cold Test Data................................................157

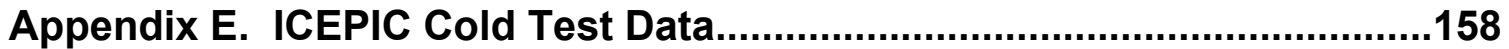

Appendix F. Simulated 6 Vane Magnetron Mode Spectrum......................159 


\section{Chapter 1}

\section{Introduction}

Magnetrons are utilized extensively for commercial and military applications requiring power levels from kW to MW [1-4]. Due to its compactness

and efficiency, the relativistic magnetron, which operates at power levels ranging from 100's of MW to GW, is one of the premier HPM sources [1]. Because high power microwaves (HPM from $100 \mathrm{MW}-1 \mathrm{GW})$ with long pulse lengths $(0.1-1 \mu \mathrm{s})$ are important to the Department of Defense and the plasma physics research community $[5,6]$, there is continued interest in improving the performance of relativistic magnetrons [7-9]. The aspects of magnetron performance in which improvement is sought include mode control, peak power, start-oscillation time, and pulse length. One promising technique that has emerged as a way to enhance magnetron performance is "magnetic priming" [10-19].

Magnetic priming is defined as N/2 azimuthal magnetic perturbations applied to an $\mathrm{N}$-cavity magnetron for rapid generation of the desired number of electron spokes, N/2, for the $\pi$-mode. In the $\pi$-mode, the RF electric field between two neighboring cavities is 180 degrees out-of-phase. This magnetic priming technique was originally demonstrated in $\mathrm{kW}$ oven magnetrons by Neculaes et al. $[10,11,14,15,19]$. Simulations using the MAGIC PIC (particle-in- 
cell) code by Jones et al. showed that the application of an idealized magnetic priming field in a relativistic magnetron yielded rapid growth of the m-mode and suppression of mode competition $[16,17,20]$. Realistic oven magnetron simulations, including straps, using ICEPIC [21] corroborate Neculaes experiments. Other magnetron priming techniques investigated in recent years include: cathode priming [20,22-25], "transparent" cathodes [26-28], electric priming at the anode [29,30], and RF priming [31-35].

In order to create the azimuthal magnetic field variations in the previous oven magnetron experiments, small rare-earth magnets were placed on the outside of the annular, permanent magnets that are standard on the devices [1013,19], as shown in Figure 1 (a) and (b). Relativistic magnetrons operate at much higer voltages and are substantially bulkier than oven magnetrons. They generally use large pulsed electromagnets to create the azimuthal magnetic field required for operation. Scaling up the original oven magnetron magnetic priming method to a relativistic magnetron would require very large external priming magnets which would exceed the physical size constraints of the magnetron experiment. Thus, in order to impose the magnetic field perturbations required for magnetic priming of relativistic magnetrons, a new technique was required. Presented in this dissertation are the results of efforts to implement new magnetic priming techniques in relativistic magnetrons. 

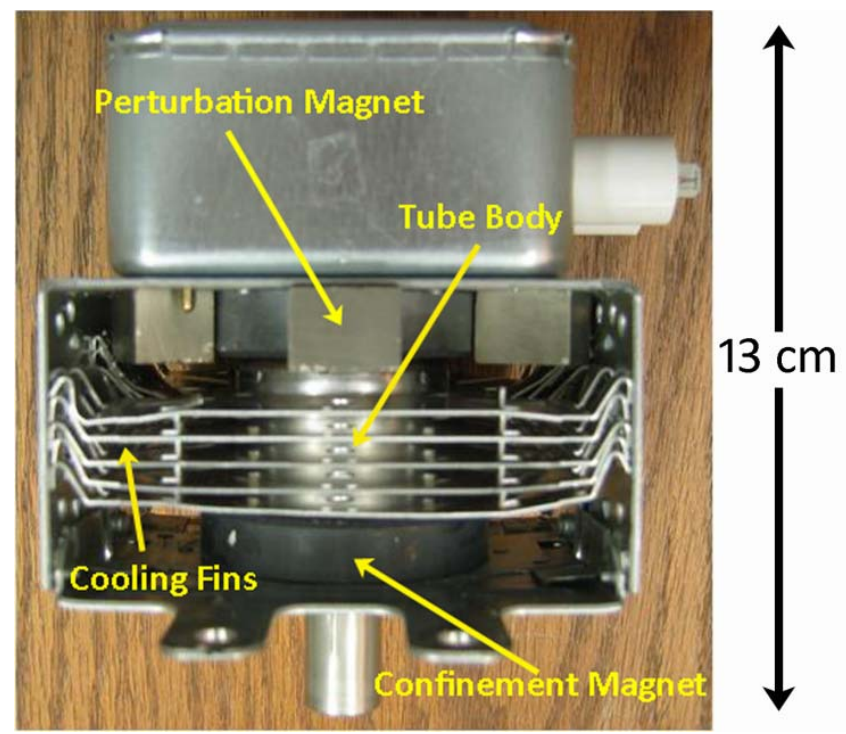

(a)

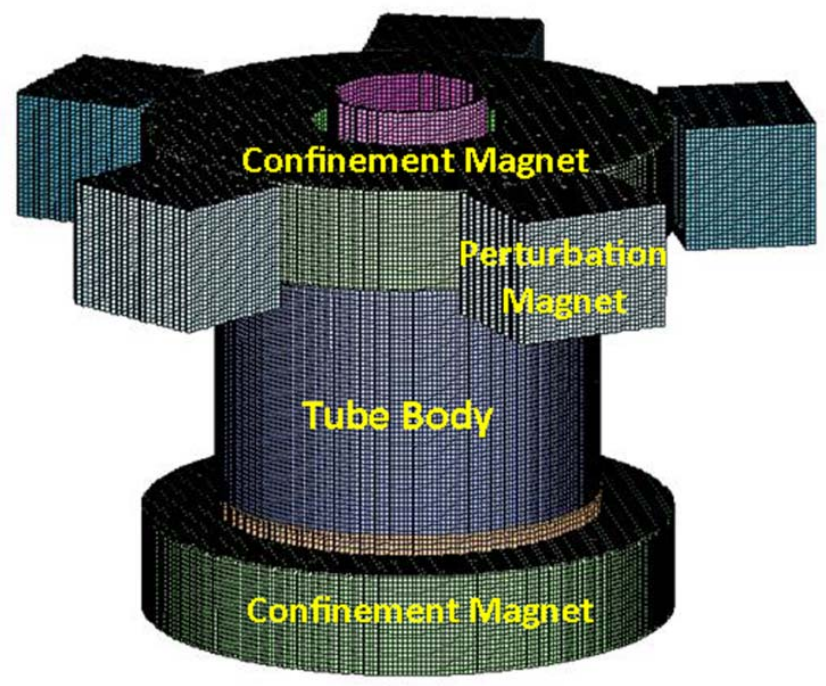

(b)

Figure 1. (a) Magnetically primed oven magnetron (after Neculaes [11]). (b) Computer model of a magnetically primed oven magnetron.

The magnetic priming concept implemented in the UM/L-3 Titan relativistic magnetron places magnetic structures within the magnetron itself. The initial embodiment of this concept involved placing magnetic structures within the cathode of the relativistic magnetron [36]. Figure 2 depicts a computer 
representation of the UM/L-3 relativistic magnetron cathode with three magnetic wires embedded within. Because of the close proximity of the priming structures to the cathode surface, the magnetic perturbations are strongest at the cathode and weakest at the anode. This configuration is the reverse of the original magnetic-priming experiments on oven magnetrons [10], where the magnetic perturbations are strongest in the anode region and decrease toward the cathode. Following the implementation of magnetic priming at the cathode of the relativistic magnetron, the feasibility of embedding magnetic structures in the anode block of the relativistic magnetron was also explored. In this configuration, the magnetic priming geometry more closely approximates the original oven magnetron magnetic-priming experiments [10].

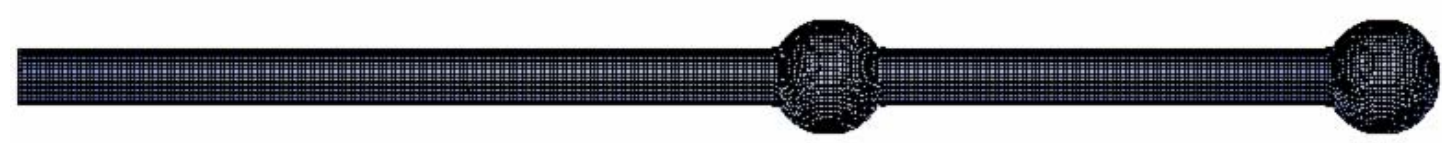

(a)

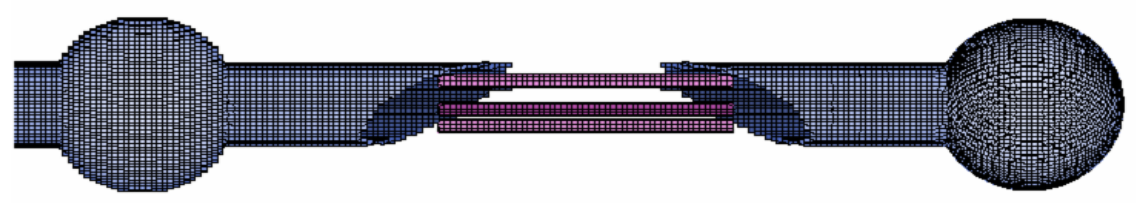

(b)

Figure 2. Computer model showing a portion of the UM/L-3 relativistic magnetron cathode with attached electrostatic end balls. (b) Close-up cutaway view of the cathode emission region showing wire locations.

During the course of the magnetic priming experiments on the UM/L-3 relativistic magnetron, it was discovered that window flashover was occurring on 
the vacuum side of the microwave windows [37]. This window flashover was limiting the microwave power and pulse width that could be extracted from the magnetron. These microwave window performance issues were studied as part of a collaboration with the Air Force Research Laboratory. The research performed in this collaboration resulted in the design and successful implementation of a solution which eliminated window breakdown in subsequent operation of the UM/L-3 magnetron. 


\section{Chapter 2}

\section{Basic Magnetron Theory and Background}

Although magnetrons have been studied for over 80 years, they have proven extremely difficult to work with analytically [2]. Prior to widespread use of PIC modeling codes, magnetron design was primarily reliant on empirical data and two equations, the Hull condition and the Buneman-Hartree condition[2$4,7,8,38,39]$.

When a an electron is immersed in a constant magnetic field it will oscillate around a guiding center with angular frequency $\omega_{c}$ at a radius $r_{L}$, (the Larmor radius). The equations for calculating $\omega_{c}$ and $r_{L}$ are given by (2.1) and (2.2), respectively,

$$
\begin{aligned}
& \Omega_{c}=\frac{|q| B}{m}, \\
& r_{L}=\frac{v_{\perp}}{\Omega_{c}},
\end{aligned}
$$

where $q$ is the particle charge, $B$ is the magnetic field strength and $v_{\perp}$ is the perpendicular component of the particle velocity.

If an electric field perpendicular to the direction of the magnetic field is also present, the particle motion will consist of both the Larmor gyration and a drift of the guiding center [40]. The $E \times B$ drift velocity of the particle] is given by equation (2.3),

$$
\boldsymbol{v}_{E \times B}=\frac{E \times B}{B^{2}} .
$$


In order to operate, magnetrons rely on the principle of synchronism. For magnetrons, synchronism refers to the condition in which the $E \times B$ drift velocity of the strongly interacting electrons within the device is equal to the phase velocity of the magnetron's operating mode. If this condition is satisfied, the electrons can transfer energy to the wave.

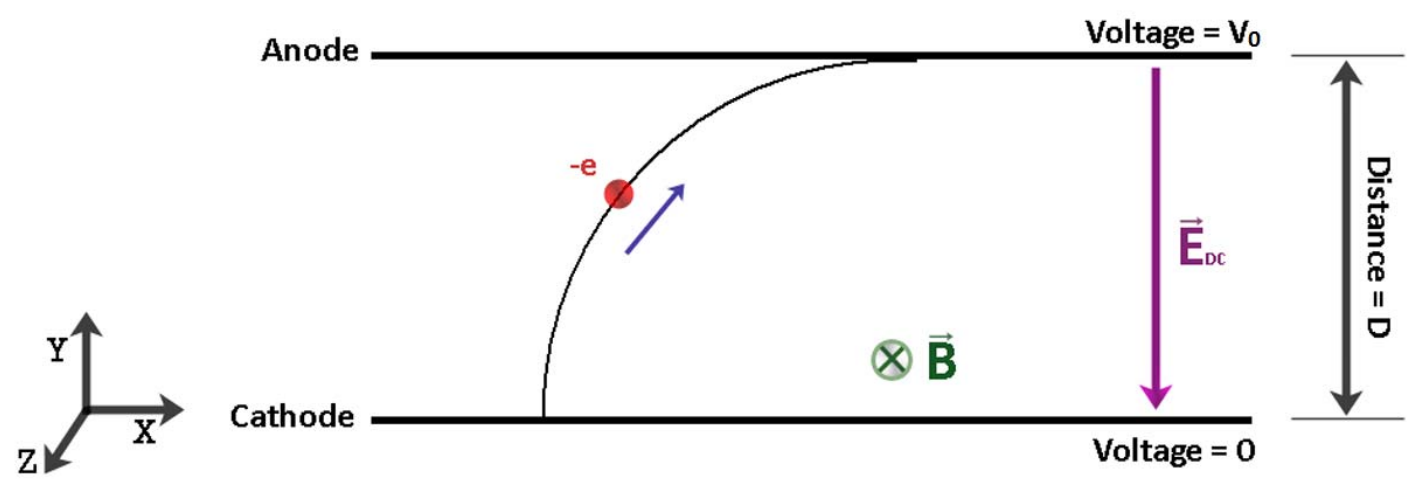

Figure 3. Uncorrugated planar magnetron, showing an electron trajectory when the Hull condition is barely satisfied.

Although most magnetrons are cylindrical devices, we will start with a simple, uncorrugated planar magnetron model, as shown in Figure 3, to demonstrate how synchronism is achieved. For the first part of this discussion, relativistic effects will be ignored. In this model, the cathode is held at ground potential (defined as 0 volts) and the anode is held at voltage $V_{0}$. The voltage differential between the anode and cathode results in a DC electric field, $E_{D C}$, in the direction indicated in Figure 3. In the case where there is no magnetic field, an electron released from rest would stream in a straight line along the $Y$ direction from its release point on the cathode to the anode. If a magnetic field is applied in the direction indicated (-Z), the centripetal acceleration caused by the 
magnetic field will result in cyclotron motion, deflecting the particle in the $X$ direction. For a given voltage, there is a specific magnetic field at which the particle's Y-directed motion will be equal to zero just as the particle touches the anode. This condition is defined as the Hull condition or Hull cutoff condition. In order to derive the Hull condition from single electron orbits, we start by invoking conservation of energy at the point at which the electron just grazes the surface of the anode (where $y=D$ ). At this point, the electron's velocity is entirely in the $x$ direction. Assuming that the electron was released from the surface of the cathode with zero initial velocity the energy conservation equation is

$$
e V=\frac{1}{2} m v_{x(y=D)^{2}} .
$$

Solving (2.4) for velocity yields

$$
v_{x(y=D)}=\sqrt{\frac{2 e V}{m_{e}}} .
$$

In order to solve for the velocity in the $\mathrm{X}$ direction at $\mathrm{y}=\mathrm{D}$, we turn to the Lorentz force equation. The Lorentz force equation, given as equation (2.6), relates the force experienced by a charged particle of a given mass to the electric and magnetic fields in which it is immersed.

$$
\boldsymbol{F}=m \dot{\boldsymbol{v}}=q(\boldsymbol{E}+\boldsymbol{v} \times \boldsymbol{B})
$$

As we are discussing force exerted on electrons and their resulting motion, $\mathrm{m}$ will be replaced with $m_{e}$, the electron mass. In the same manner, the charge, $q$, will be replaced with $-\mathrm{e}$, the elementary charge of an electron $(\mathrm{e}>0)$. The DC electric field, $\mathbf{E}_{\mathrm{DC}}$, can be exchanged with the quantity $-\mathrm{V} / \mathrm{D} \hat{\mathbf{x}}$. Equation (2.4) can then be separated into its constituent vector equations 


$$
\begin{gathered}
m_{e} \dot{v}_{x}=e v_{y} B \\
m_{e} \dot{v}_{y}=\frac{e V}{D}-e v_{x} B \\
m_{e} \dot{v}_{z}=0
\end{gathered}
$$

Integrating both sides of (2.7) with respect to time and solving for $v_{x}$ yields

$$
v_{x}=\frac{e B}{m_{e}} y+\text { constant }
$$

Because the particle is assumed to start at rest from the cathode, (located at $y=$ 0 ), it is apparent that the constant in (2.8) is equal to zero, leaving only

$$
v_{x}=\frac{e B}{m_{e}} y .
$$

Evaluating (2.11) at $y=D$, substituting the result into (2.5) and solving for $V$ results in (2.12), the equation defining the Hull condition for the magnetic field at a given voltage, $\mathrm{V}$,

$$
B_{H u l l}=\sqrt{\frac{2 m_{e} V}{e D^{2}}} .
$$

For a given magnetic field, $\mathrm{B}$, electrons will not reach the anode if $\mathrm{V}<\mathrm{V}_{\text {Hull, }}$ where

$$
V_{\text {Hull }}=\frac{1}{2} \frac{e}{m_{e}} B^{2} D^{2} .
$$

If the applied magnetic field is greater than that required by the Hull condition, the electron will be redirected back toward the cathode. If multiple electrons are emitted, multiple overlapping electron trajectories can be generated, as shown in Figure 4. 


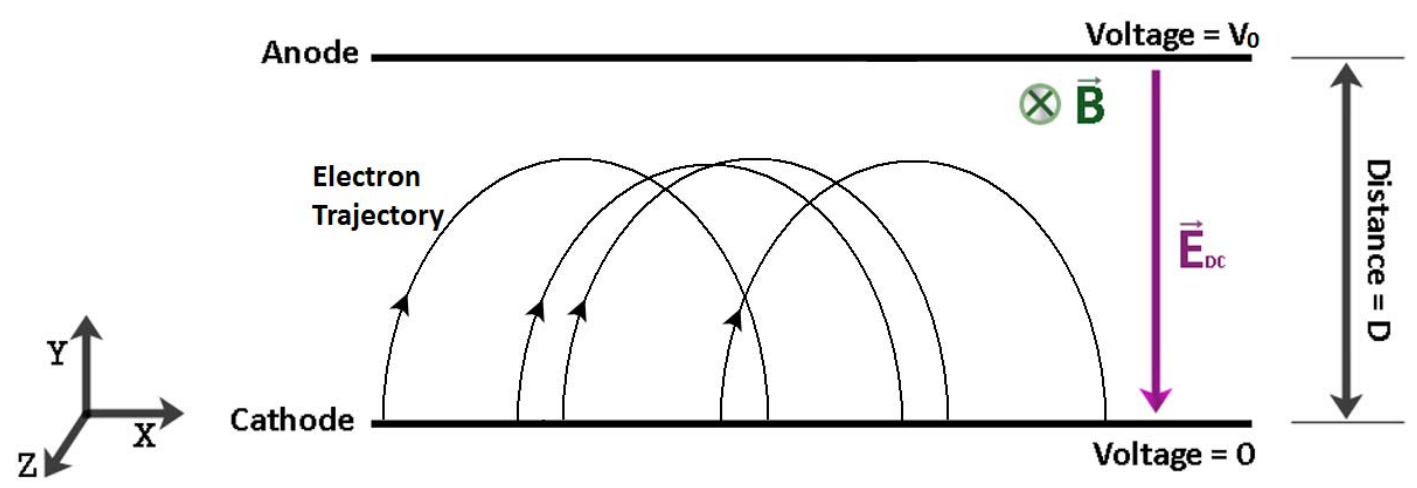

Figure 4. Uncorrugated planar magnetron with an applied magnetic field greater than required for Hull cutoff, showing multiple electron trajectories.

For a large number of electrons, the cycloidal orbits result in a net current flow in the $\mathrm{X}$ direction $[38,41,42]$. These cycloidal orbits are highly unstable, as argued by Slater [38], and demonstrated via particle-in-cell simulations in [43-46]. The likely final state of the collective electron motion is a laminar flow superimposed on a weak turbulent background [44]. This laminar electron flow, illustrated in Figure 5, is called the Brillouin flow [41]. In simulations, if there are even slight perturbations to the system, the transition from Slater orbits into a Brillouin flow occurs almost instantly [43-46]. It is from this Brillouin flow model that we will develop the Buneman-Hartree resonance condition for synchronism [2]. 


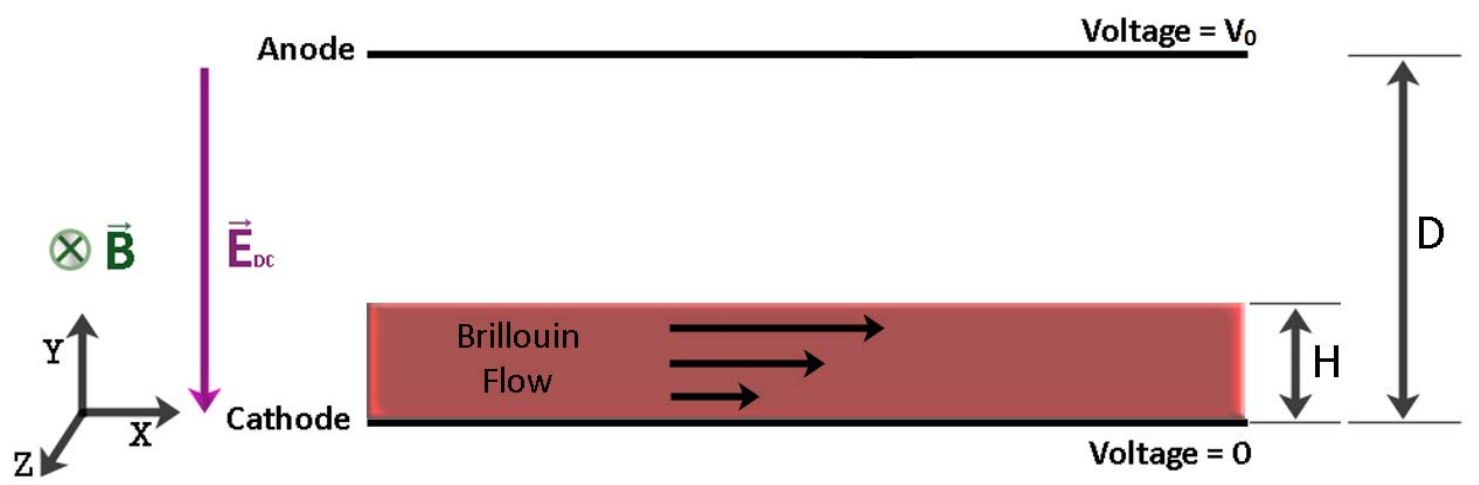

Figure 5. Uncorrugated planar magnetron with laminar (Brillouin) electron flow.

First, let $v=\hat{x} v(y)$ be the electron flow velocity. Equating it to the $E \times B$ drift, we get

$$
v=\frac{E(y)}{B_{0}}=\frac{\partial V}{\partial y} \frac{1}{B_{0}}
$$

Invoking conservation of energy for an electron yields

$$
e V=\frac{1}{2} m_{e} v^{2}
$$

Differentiating (2.14) gives:

$$
e \frac{\partial V}{\partial y}=m_{e} v \frac{\partial v}{\partial y}
$$

From equations (2.13) and (2.15), we obtain:

$$
\frac{\partial v}{\partial y}=\frac{e B_{0}}{m_{e}} \equiv \Omega
$$

and, therefore

$$
\mathrm{v}(\mathrm{y})=\Omega \mathrm{y} .
$$

where $\mathrm{eB} / \mathrm{m} \equiv \Omega$ is the electron cyclotron frequency. Note that the profile of the Brillouin flow described by (2.16) and illustrated in Figure 5 exhibits constant velocity sheer. 
Substituting (2.17) into (2.14) gives

$$
V(y)=\frac{1}{2} \frac{m_{e}}{e} \Omega^{2} \mathrm{y}^{2} .
$$

In this geometry, Poisson's equation can be written as

$$
-\frac{\partial^{2} V(y)}{\partial y^{2}}=\frac{\rho}{\epsilon_{0}}=-\frac{m_{e}}{e} \Omega^{2} .
$$

Equation (2.18) provides the characteristic scaling for the Brillouin flow:

$$
\omega_{p e}{ }^{2}=\Omega^{2},
$$

where $\omega_{p e}$ is the electron plasma frequency. Because of this, the external magnetic field fixes the density scale in crossed-field devices, including the magnetron.

Referring to Figure 5, for $\mathrm{y}$ between $\mathrm{H}$ and $\mathrm{D}$, the electric field is equal to $\mathrm{E}(\mathrm{H})$, which is obtained by differentiating (2.18) with respect to $\mathrm{y}$ and setting $\mathrm{y}=$ $\mathrm{H}$ :

$$
E(H)=\frac{m_{e}}{e} \Omega^{2} \mathrm{H},
$$

assuming space charge limited emission $(E(0)=0)$. The total voltage differential across the gap is the sum of the voltage increase from $y=0$ to $y=H$ and from $y$ $=\mathrm{H}$ to $\mathrm{y}=\mathrm{D}$ :

$$
V_{0}=\frac{1}{2} m_{e} \frac{(\Omega H)^{2}}{e}+(D-H) \frac{m_{e}}{e} \Omega^{2} \mathrm{H} .
$$

Equation (2.21) can be simplified into the form

$$
V_{0}=\frac{m_{e}}{e} \Omega^{2} \mathrm{HD}-\frac{1}{2} \frac{\mathrm{m}_{\mathrm{e}}}{\mathrm{e}} \Omega^{2} \mathrm{H}^{2} .
$$

In order to proceed further in this derivation, we must now include a slow wave structure (in this case, corrugations in the anode $[3,38]$ ) and assume that 
an electromagnetic wave is propagating in the $\hat{x}$ direction through the planar magnetron with phase velocity $\mathrm{v}_{\mathrm{ph}}$, as depicted in Figure 6 .

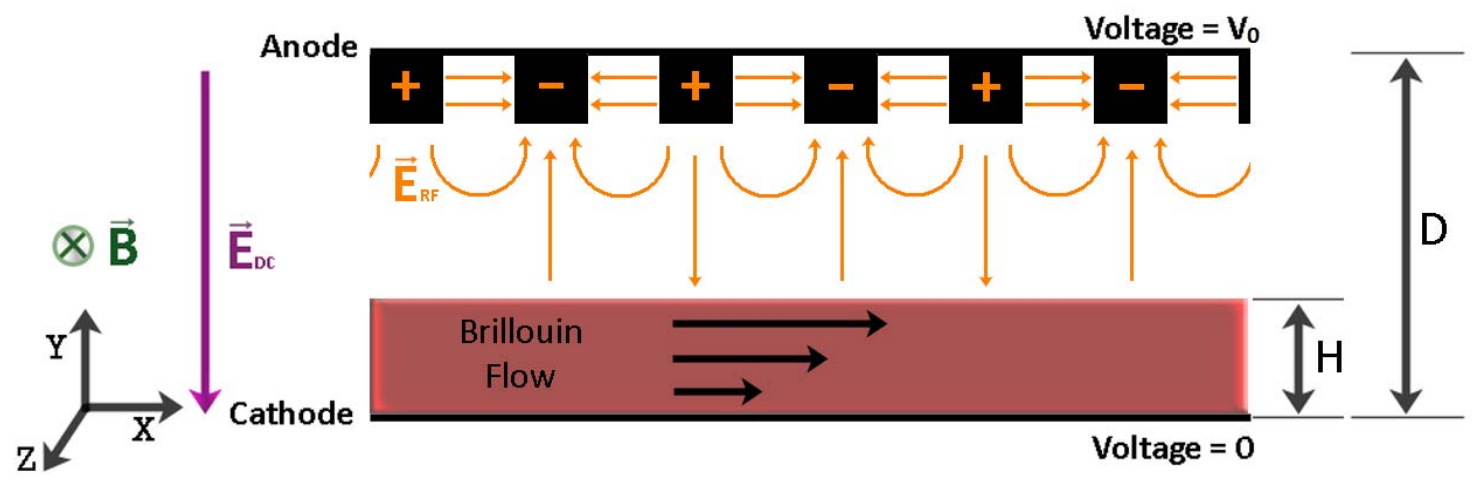

Figure 6. Corrugated planar magnetron showing RF wave and electron Brillouin flow.

Recalling (2.17) and requiring that, as a result of the synchronism condition, at the hub height $(y=H)$ the electron drift velocity must be equal to the phase velocity of the wave gives the relation:

$$
v_{p h}=\Omega H .
$$

Introducing (2.23) into (2.22), remembering that $\Omega=e B / m_{e}$, and renaming $V_{0}$ as $\mathrm{V}_{\mathrm{BH}}$ yields equation (2.24), the Buneman-Hartree resonance condition:

$$
V_{B H}=B D v_{p h}-\frac{1}{2} \frac{m}{e} v_{p h}^{2} .
$$

The Hull and Buneman-Hartree conditions can be plotted together to form a set of operating curves for a particular magnetron. Figure 7 depicts a generic set of magnetron curves. Note that although only one curve is shown, the Buneman-Hartree condition denotes a family of curves, one for each mode supported by the slow wave structure of the magnetron. 


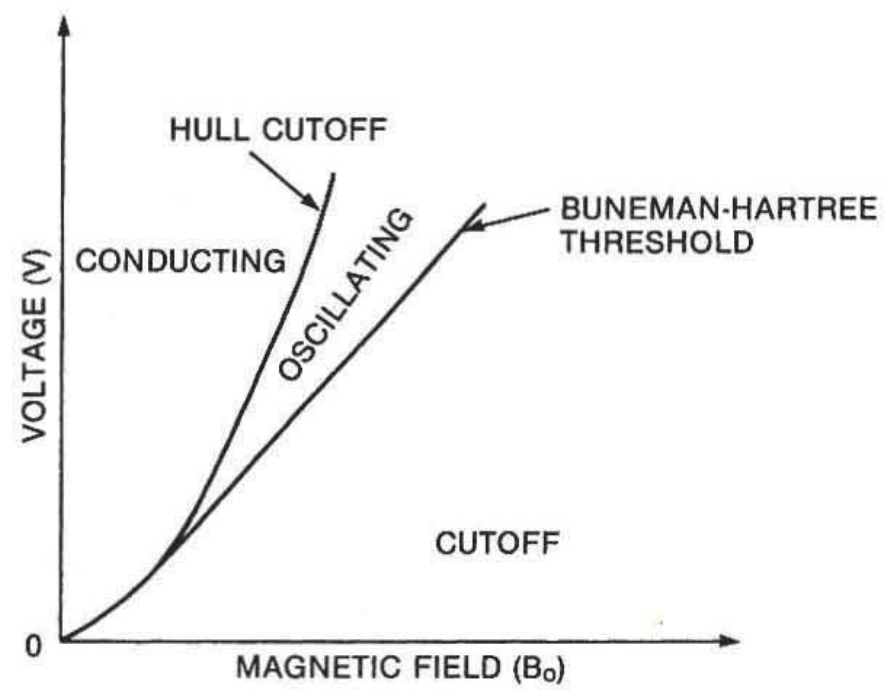

Figure 7. Generic diagram of magnetron operating curves, showing the Hull cutoff and Buneman-Hartree resonance condition. Reproduced from Ref. [2].

As shown in [4], and quoted in [2], [7], and [8], when relativistic and cylindrical effects are included the Hull condition becomes

$$
B_{H u l l}=\left(\frac{m_{0} c}{e D^{*}}\right)\left[\frac{2 e V}{m_{0} c^{2}}+\left(\frac{e V}{m_{0} c^{2}}\right)^{2}\right]^{\frac{1}{2}},
$$

and the Buneman-Hartree resonance condition becomes

$$
V_{B H}=B_{0} D^{*} v_{p h}-\frac{m_{0} c^{2}}{e}\left\{1-\left[1-\left(\frac{v_{p h}}{c}\right)^{2}\right]^{\frac{1}{2}}\right\},
$$

In (2.25) and (2.26), $m_{0}$ is the rest mass of the electrons. $D^{*}$ is the effective gap of the cylindrical magnetron and is calculated as $D^{*}=\left(r_{a}^{2}-r_{c}^{2}\right) /\left(2^{*} r_{c}\right)$ where $r_{a}$ is the anode radius and $r_{c}$ is the cathode radius. Equations (2.25) and (2.26) reduce to (2.12a) and (2.12b), respectively, in the planar, non-relativistic limit. It is important to note that the derivations for the Buneman-Hartree conditions are based on three important assumptions [2]: (a) the externally applied magnetic field is azimuthally symmetric; (b) the RF field amplitude is low enough such that 
the energetics of the orbital motion is not affected; (c) the fields are independent of time. In actual experiments, these assumptions are not necessarily valid $[2,8]$. In the case of magnetic priming, assumption (a) is, by definition, violated. While the Buneman-Hartree condition, as formulated in (2.25) and (2.26) cannot be expected to yield precise operating curves for a given magnetron, it does provide a guideline for what mode is likely to be dominant in the magnetron for given voltage and magnetic field values.

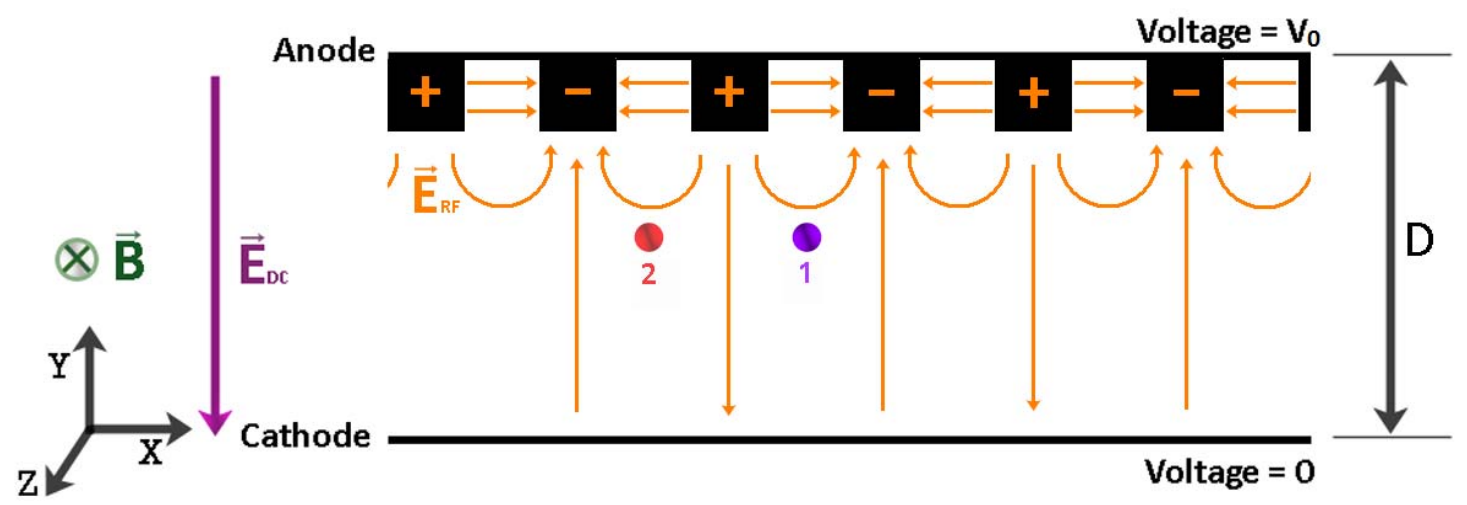

Figure 8. Corrugated planar magnetron operating in the m-mode. Two electrons are shown, each in a different phase of the RF wave.

In order to qualitatively describe how energy is transferred from the drifting electrons to the wave we will refer to Figure 8. The red and purple points represent two electrons, denoted 1 and 2, each in a different phase of the RF wave propagating through the magnetron [2]. Both particles are traveling to the right. It is assumed that the $\mathrm{E} \times \mathrm{B}$ drift of the electrons is in synchronism with the phase velocity of the wave, which travels rightward in Figure 8 . The RF wave has a phase shift of $\pi$ between each of the cavities for the m-mode. Electron 1 is decelerated by the RF electric field of the wave. The decelerating electron does 
work on the wave and the RF wave grows. Additionally, the $E_{R F} \times B$ drift is oriented in the $+Y$ direction and thus draws the electron closer to the anode, where the RF wave is strongest. This causes the electron to lose even more energy to the wave. Note that as electron 1 moves toward the anode, it loses potential energy, and this potential energy is converted to RF energy. Electron 2, on the other hand, is accelerated by the RF electric field. It is in a position to gain kinetic energy at the expense of the wave. Because the $E_{R F} \times B$ drift of electron 2 is in the $-Y$ direction, the electron is forced back toward the cathode, where the RF electric field is weakest. The overall amount of energy lost to the RF wave by electron 1 is greater in magnitude than the amount of energy electron 2 gains from the RF wave. The combination of these effects results in a net loss of kinetic and potential energy by the electrons and a net energy gain by the RF. This effect can be referred to as RF focusing.

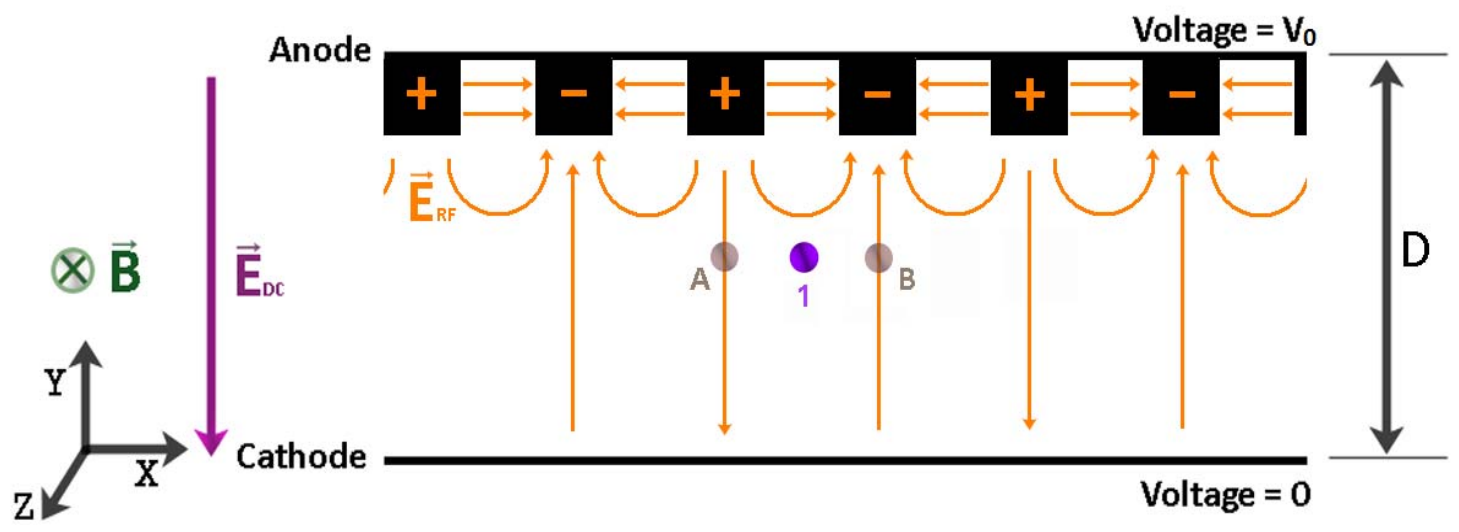

Figure 9. Corrugated planar magnetron. An electron is shown in phase with the wave. Positions $A$ and $B$ are shown as the reference positions.

Figure 9 will be used to demonstrate another phenomenon exhibited by magnetrons called phase focusing. Suppose the E x B drift velocity of electron 1 
is slightly slower than the phase velocity of the wave. In this case, at some future time, the position of the electron, relative to the wave, will slowly approach position A. At position A, the RF electric field is aligned in the same direction as the DC electric field, causing an increase in the overall electric field and, consequently, an increase in the electron's $E \times B$ drift. This effectively brings the relative position of the electron from position $A$ back toward the original position of electron 1. If, however, the electron $E \times B$ drift velocity is slightly faster than the phase velocity of the wave, the electron will gradually move toward position $\mathrm{B}$, with respect to the wave. At position $\mathrm{B}$, the RF electric field is aligned in the opposite direction of the DC Electric field add, resulting in partial cancellation of the DC electric field. This reduction in the total electric field acting on the electron results in a reduction of the $E x B$ drift velocity of the particle. This effect causes the relative position of the electron to drop back toward the original position of electron 1. Note that it is electron 1 that gives energy to the RF wave. A similar argument shows that electron 2 (in Figure 9) has a tendency to be swept to the phase of electron 1, if electron 2 has not already struck the anode.

The combination of the RF and phase focusing effects tend to ensure only electrons favorable (of the correct position and drift velocity, such as electron 1) to the growth of the RF wave remain in the interaction region. These focusing effects are the primary reason some magnetrons can operate at efficiencies in excess of $80 \%[2,47]$.

Due to the effects of RF focusing and phase focusing, the electron Brillouin hub is distorted into a series of spokes. Figure 10 (a) shows a simulated 
planar magnetron prior to the start of oscillation. The electrons, comprising the laminar Brillouin flow are shown in red. Figure 10 (b) shows the same magnetron later in time, after oscillation has started. The spokes, formed by "electron 1", are clearly visible in every other cavity (defined as $\pi$-mode). In both magnetrons depicted in Figure 10, the electron drift motion and the RF phase velocity are both toward the left.

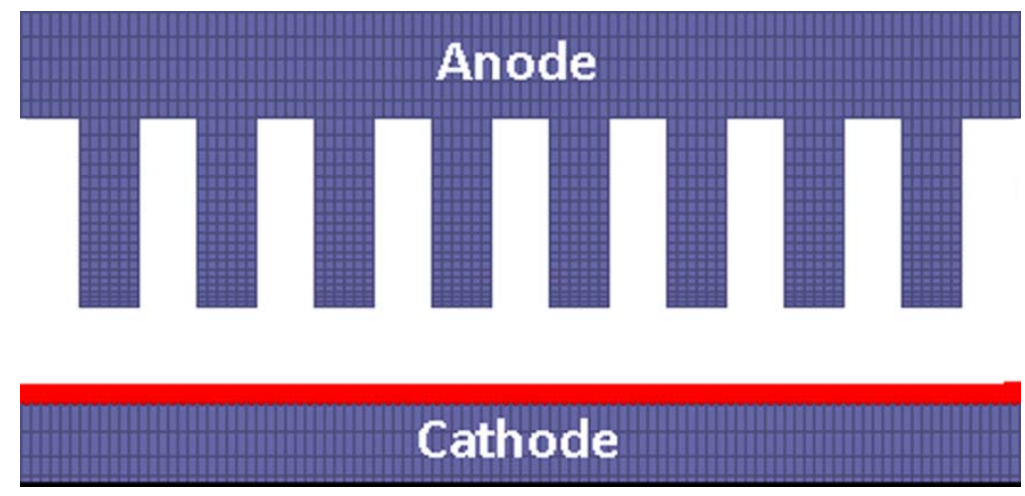

(a)

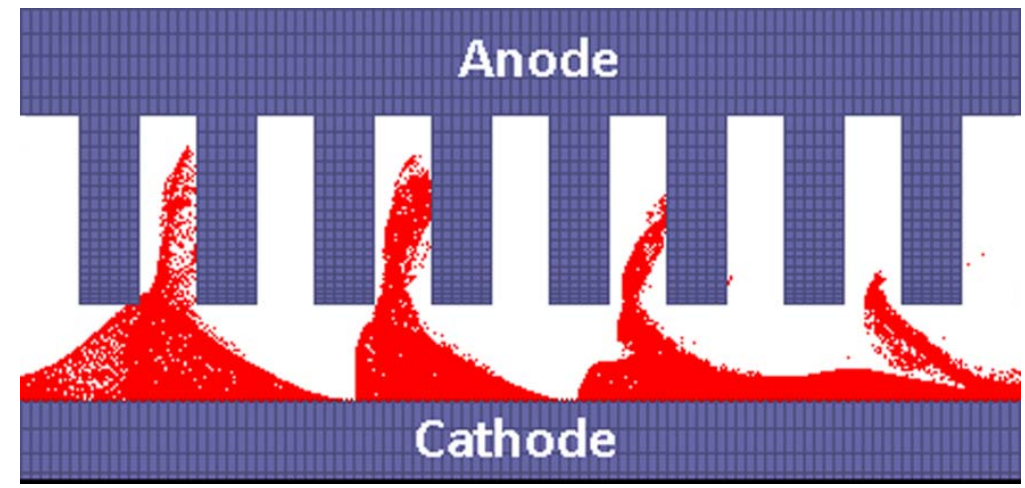

(b)

Figure 10. Corrugated planar magnetron simulation. (a) Laminar flow prior to start of oscillation. (b) Spoke formation after the start of oscillation. Here, the external magnetic field points out of the plane of the paper. 
Shifting to cylindrical geometry, the anode and cathode structures take on the form shown in Figure 11. In this figure, the anode block is denoted by "A" and the cathode is denoted by "K". For the given electric and magnetic field directionality, the electron $\mathrm{E} \times \mathrm{B}$ drift will be in the counter-clockwise direction.

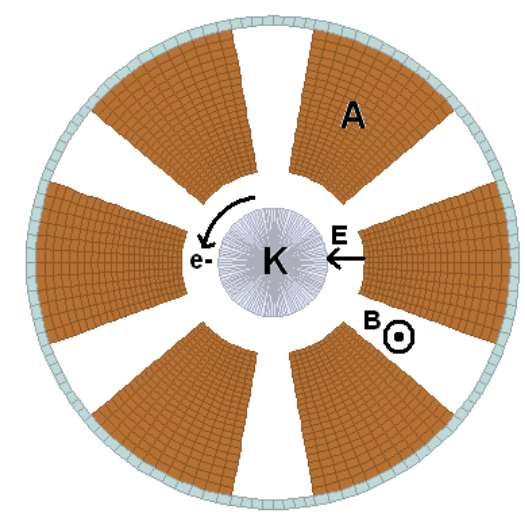

Figure 11. A 6-cavity cylindrical magnetron representation, showing applied electric and magnetic field orientations as well as electron drift direction.

Figure 12 (a) depicts a 6-cavity cylindrical magnetron in the pre-oscillation phase, showing the dominanance of the Brillouin hub (over the cycloidal orbits). As with the planar magnetron, once oscillation starts, the Brillouin hub is distorted into a series of spokes, as shown in Figure 12 (b). In this case, the magnetron is operating in the $\pi$-mode ( $\mathrm{N} / 2$ electron spokes, where $\mathrm{N}$ is the number of anode cavities). It is possible to achieve frequency "tunability" in a fixed geometry magnetron by altering the voltage and magnetic field settings to favor different oscillation modes; however, in general, magnetrons are designed to operate in one specific mode (typically the m-mode) for efficiency or power considerations. 


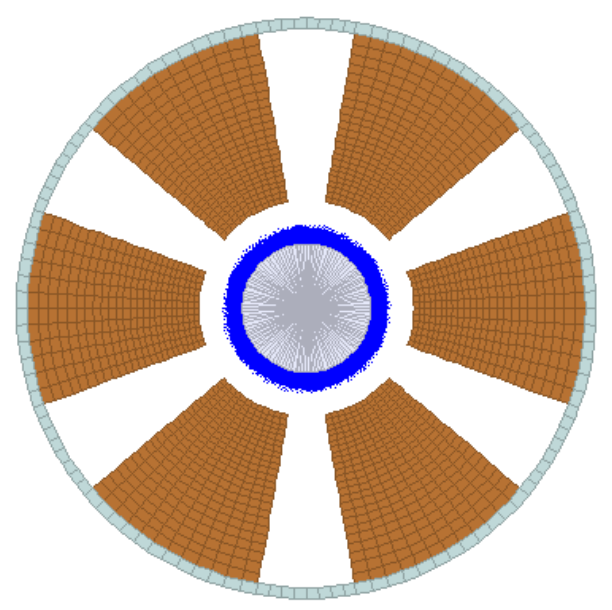

(a)

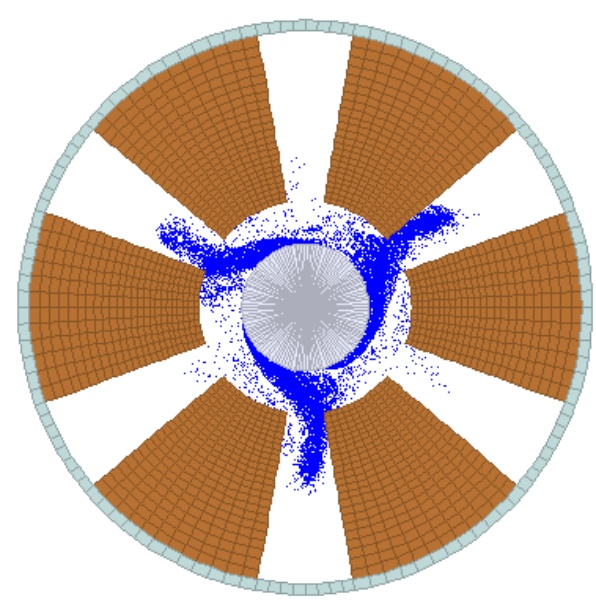

(b)

Figure 12. A 6-cavity cylindrical magnetron simulation. (a) Laminar Brillouin flow prior to start of oscillation. (b) Spoke formation after the start of oscillation.

When magnetron oscillation modes are close in frequency, the magnetron can exhibit a behavior called "mode competition" or "mode hopping", where the magnetron will shift between two different modes of oscillation. Generally, this behavior is unwanted as it adversely affects power output and frequency stability. Steps may be taken during the design phase to include structures that will favor desired modes and suppress the other unwanted modes. One such mode 
selection technique, used in microwave oven magnetrons, is called strapping $[4,38]$. Strapped magnetrons have conducting rings, or "straps" shorting every other anode vane together (i.e. odd vanes shorted together and even vanes shorted together). This technique strongly favors the magnetron to operate in the T-mode, where electric field of the RF wave alternates by 180 degrees from one anode cavity to the next. Figure 13 shows a cut-away view of a strapped oven magnetron. The RF fields supported by the straps must be a significant fraction of the RF field of the operating mode of the magnetron in order to affect the operating frequency $[4,38,48]$. Large RF electric fields in the strapping region are likely to cause arcing. Thus, traditional strapping methods cannot be used in relativistic magnetrons [48].

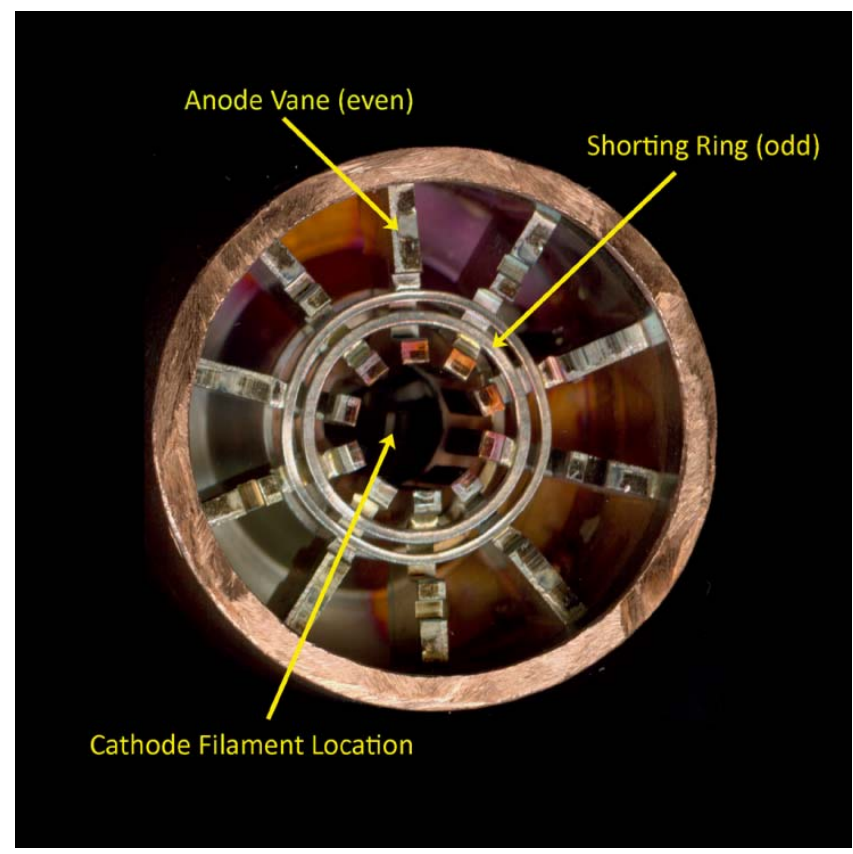

Figure 13. Cut-away view of an oven magnetron, showing shorting rings (straps) and other internal structures. 
Another technique used to increase mode control, as well as enhance other aspects of magnetron performance such as reducing the time to start oscillation, is magnetic priming [10-15]. This technique introduces systematic azimuthal variations in the axial magnetic field of the magnetron. Following the experimental discovery of magnetic priming, simulation and analytical work by Neculaes et al. [49] showed that cycloidal orbits of electrons in a gap with crossed electric and magnetic fields, such as a magnetron, lead to rapid spoke formation if the external magnetic field has periodic variations. This rapid spoke formation is due in part to kinematic bunching caused by a parametric instability in the electron orbits in the periodically perturbed magnetic field [50]. Further simulation and experimental work regarding magnetic priming, as applied to relativistic magnetrons, is presented in the following chapters. 


\section{Chapter 3}

\section{Magnetic Priming Simulations}

The initial simulation research involving magnetic priming of a relativistic magnetron performed by Jones et al. [16-18], utilized an idealized magnetic priming field in the simulations. The idealized field imposed was of the form,

$$
B_{Z}(\theta)=B_{0} *\left\{1+\frac{\alpha}{2} \sin [n(\theta+\phi)]\right\},
$$

where $B_{0}$ is the base magnetic field, $\alpha$ is the magnetic priming strength, $n$ is the number of azimuthal perturbations, and $\phi$ is the phase of the magnetic field with respect to the anode vanes. Values for $\alpha, \mathrm{n}$, and $\phi$ used in Jones' simulations were $0.3,3$, and 0 , respectively. It is evident from equation (3.1) that the idealized magnetic perturbations in $B_{Z}$ did not vary as a function of radius from the cathode or location along the z-axis. Because the magnetic perturbations were to be created experimentally by finite structures in the cathode and anode, a more physically realizable magnetic priming field was needed for use in the simulations in order to test the feasibility of the proposed magnetic priming scheme.

Additional magnetic priming simulation research was performed by Luginsland, et al. [21], emphasizing the authenticity of the strapped microwave oven magnetron data in Neculaes' experiments $[10,19]$. These simulations were 
fully three dimensional and utilized physically realistic magnetic priming geometries, but were limited to non-relativistic regimes due to the strapping schemes used in oven magnetrons.

\subsection{Magnetostatic Simulation Setup and Results}

In order to determine the effectiveness of the wire-based magnetic priming schemes for the relativistic magnetron that is studied in this dissertation, the proposed structures were modeled using finite difference based magnetostatics software. The magnetostatic simulations for this project were performed using the Magnum 1.0 software suite produced by Field Precision [51]. The ambient magnetic field was formed using two sets of current loops designed to simulate the actual electromagnets used in the experiment, as shown in Figure 14 (a) and (b). The ambient B field amplitude was set to be slightly higher than the experimental magnetic field due to the fact that the simulations would use a larger cathode than the experiment $(2.2 \mathrm{~cm}$ diameter in the simulations versus $1.27 \mathrm{~cm}$ diameter in the experiment) for reasons related to computational time that will be explained further in the next section. 


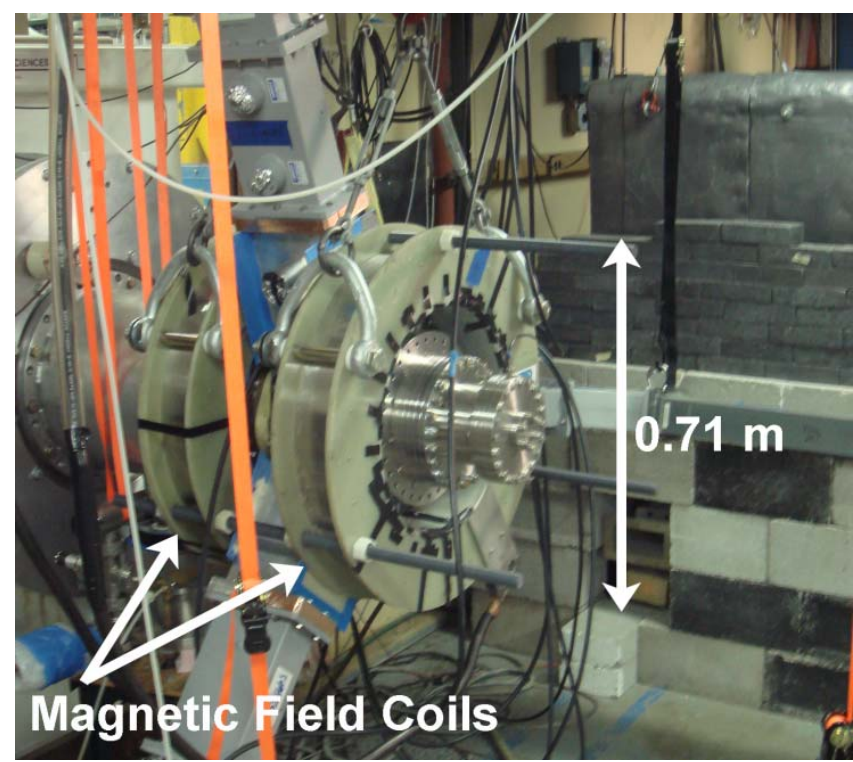

(a)

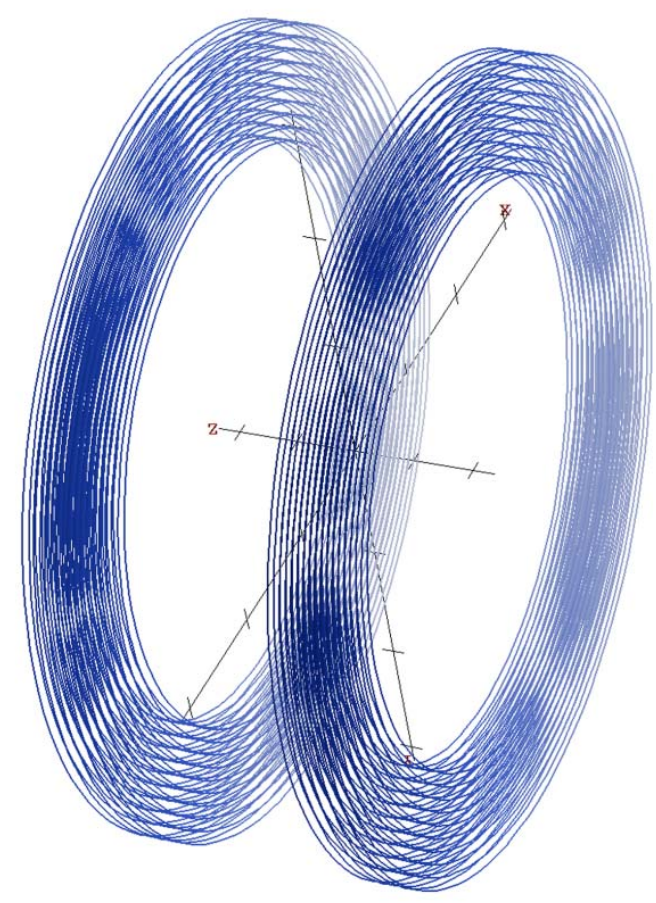

(b)

Figure 14. (a) Magnetic field coils on the UM/L-3 relativistic magnetron experiment. (b) Magnetic field coil representation in the Field Precision Magnum magnetostatics software. 
In addition to an unprimed baseline case (no magnetic perturbations), the cases of magnetic priming at the cathode ( 3 magnetic wires embedded in the cathode, spaced 120 degrees apart), magnetic priming at the anode ( 3 magnetic wires embedded in the anode, spaced 120 degrees apart), and combined magnetic priming at the cathode and anode ( 3 magnetic wires embedded in the cathode and 3 magnetic wires embedded in the anode) were simulated. Simulations were performed for various wire lengths. In the simulations, the cathode, anode, and combined magnetic priming cases utilized wires with magnetic permeability values of 500 and 1000 in order to achieve perturbation strengths on the order of those used by Jones [16-18] close to the wires. Nickeliron alloys such as Mu-metal and Permalloy can have relative permeabilities as high as 100,000; however, as these materials saturate, their relative permeability values drop rapidly [52]. Although current versions of the Magnum software can address hysteretic effects, the Magnum 1.0 software used for these simulations does not handle self-consistent magnetic saturation of materials. For these reasons, all magnetic wires are simulated as linear magnetic materials (no hysteretic effects).

The results from the baseline case, shown in Figure 15 and Figure 16, show the expected, nearly uniform field created by the magnetron's simulated electromagnets. Fields range from 3.45 kGauss at the cathode surface to 3.48 kGauss at the back wall of the magnetron. 


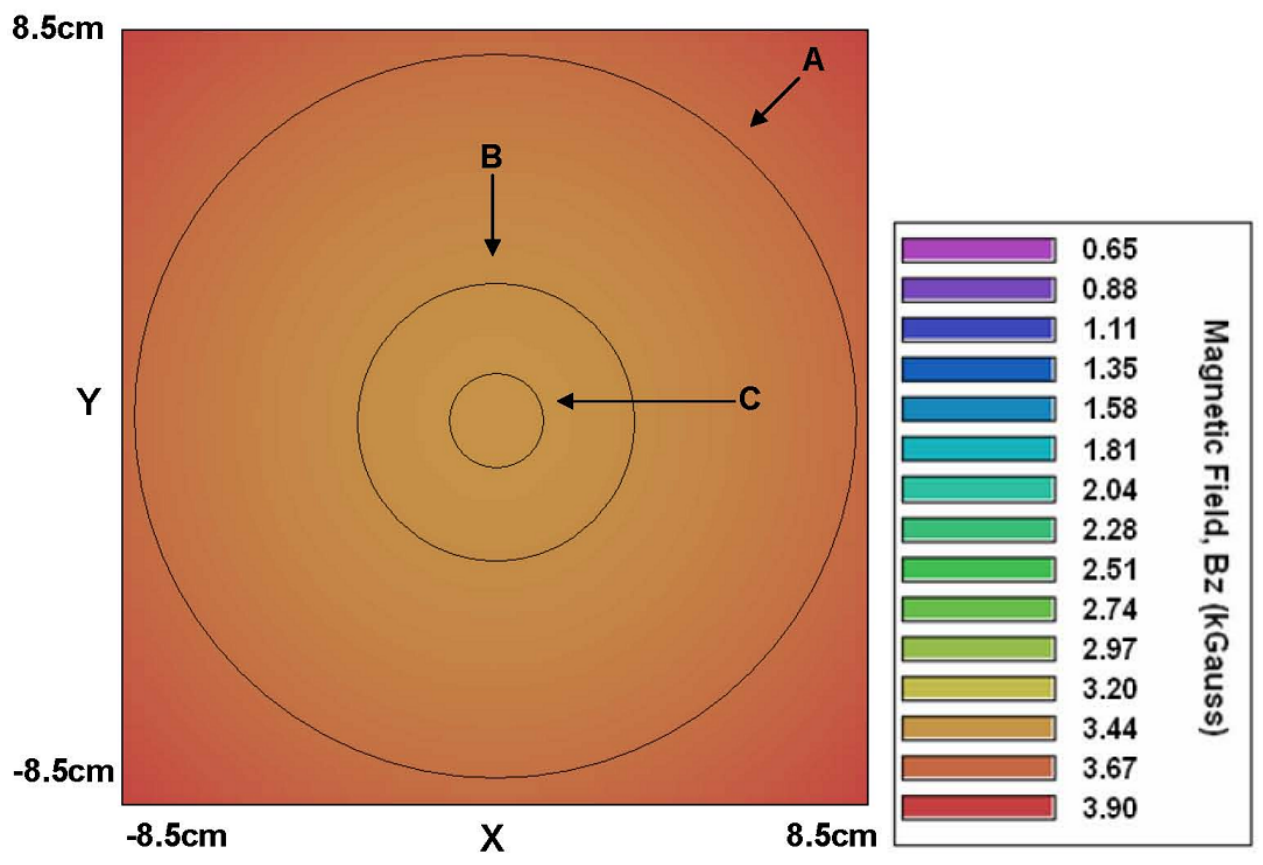

Figure 15. Contour plot of the axial magnetic field for the baseline case (no magnetic priming) A: Anode backwall (radius $=8.3 \mathrm{~cm}$ ), B: Anode block inner boundary (radius = $3.2 \mathrm{~cm}$ ), C: Cathode boundary (radius $=1.1 \mathrm{~cm}$ ).

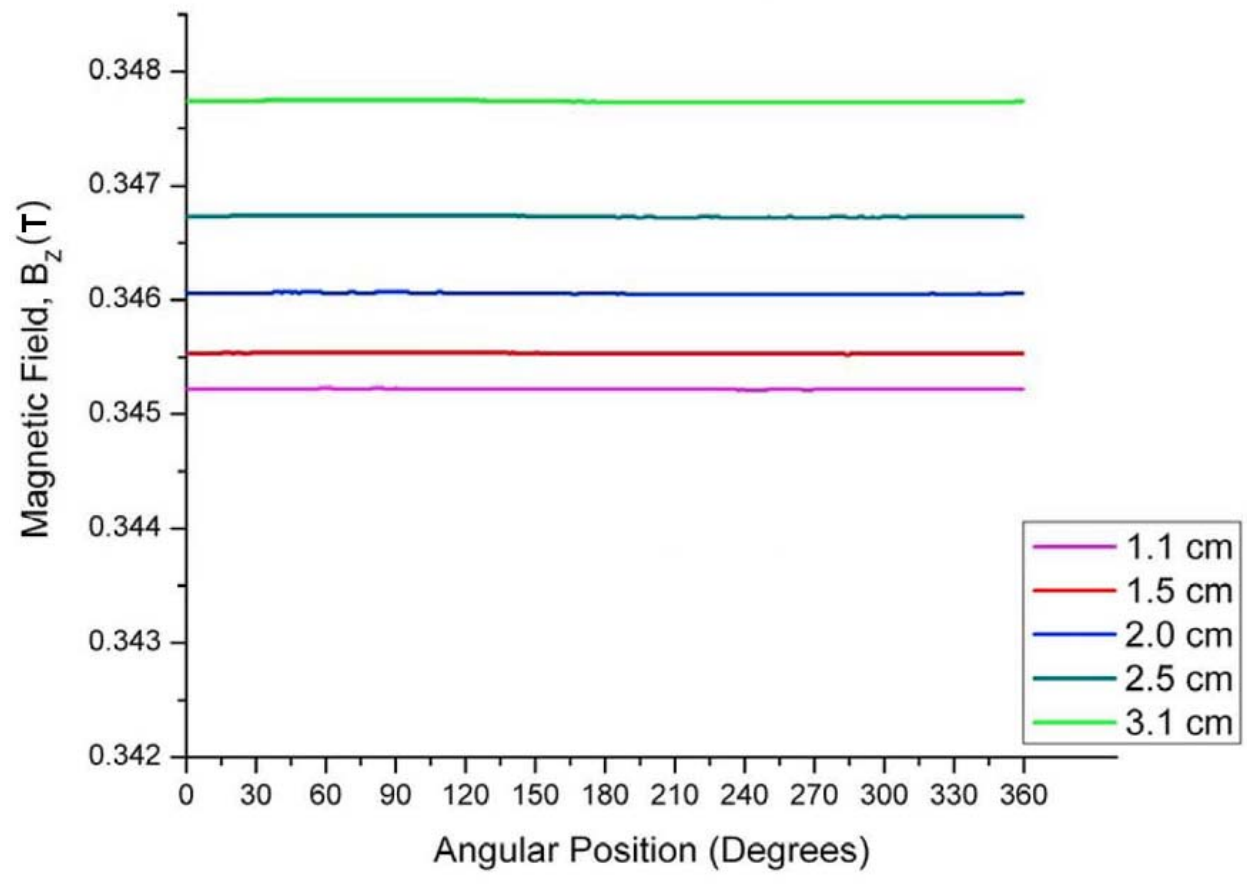

Figure 16. Axial magnetic field as a function of angle at radii $1.1 \mathrm{~cm}-3.1 \mathrm{~cm}$ (cathode radius $\mathbf{~} 1.1 \mathrm{~cm}$ ) for Baseline case (no magnetic priming). 
As would be expected, the case using wires embedded in the cathode exhibited very strong perturbations at the surface of the cathode which rapidly reduced in amplitude as radius increased. The data from the cathode wire case are displayed in Figure 17 and Figure 18. The location and cross-sectional area of the cathode wires are represented by the three white circular areas within the cathode boundary ring (ring C).
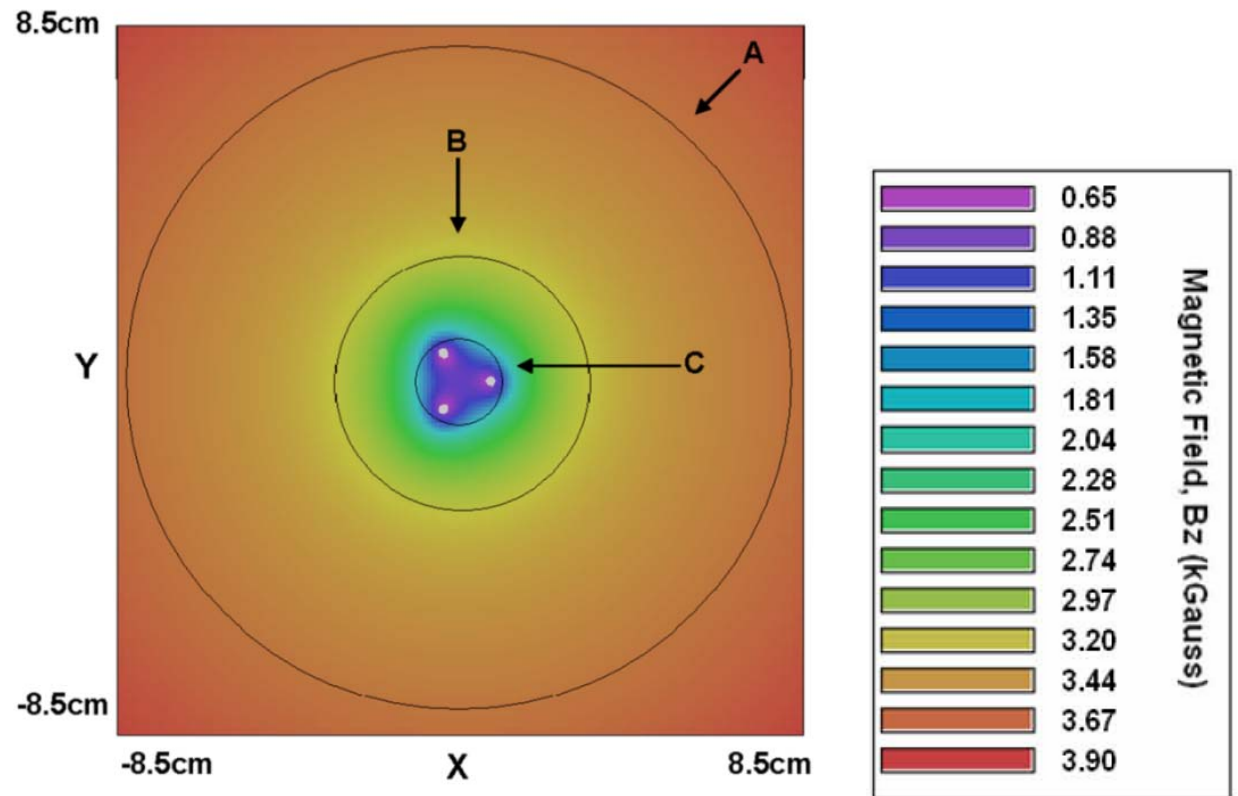

Figure 17. Contour plot of the axial magnetic field for the case of magnetic priming at the cathode. Wire length $=4 \mathrm{~cm}$. Wire relative magnetic permeability $=1000$. A: Anode backwall (radius $=8.3 \mathrm{~cm}$ ), B: Anode block inner boundary (radius $=3.2 \mathrm{~cm}), \mathrm{C}$ : Cathode boundary (radius $\mathbf{= 1 . 1} \mathrm{cm})$. 


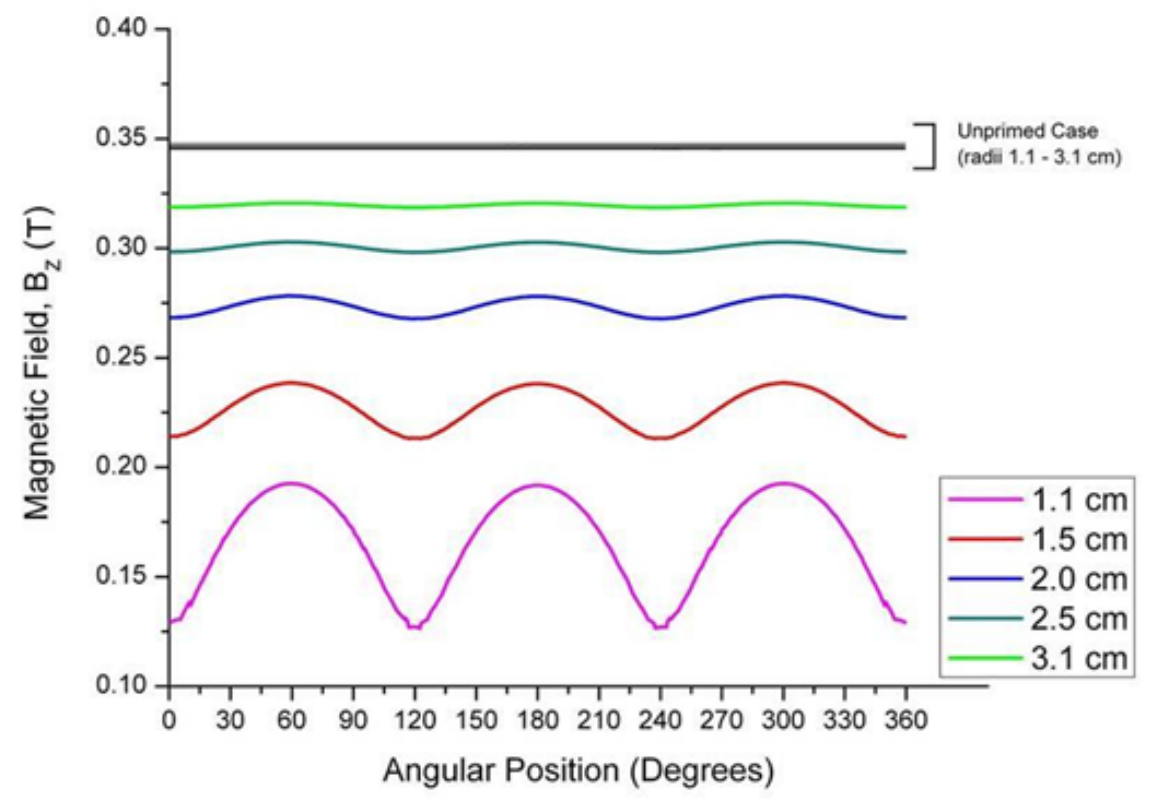

Figure 18. Axial magnetic field, $B_{Z}$, as a function of angle at Radii $1.1 \mathrm{~cm}-3.1 \mathrm{~cm}$ (cathode radius $=1.1 \mathrm{~cm}$, wires $\mu_{\mathrm{r}}=1000$ ) for the case of magnetic priming at the cathode. Unprimed (baseline) case shown for comparison.

In the case of magnetic priming at the anode, Figure 19 and Figure 20, a very weak perturbation is seen at the cathode surface. The magnitude of the perturbation grows rapidly as the anode block boundary is approached. When compared to the unprimed case (also plotted on Figure 20), the anode-magnetic priming field profile shows an overall suppression of the axial magnetic field due to the presence of the wires. 


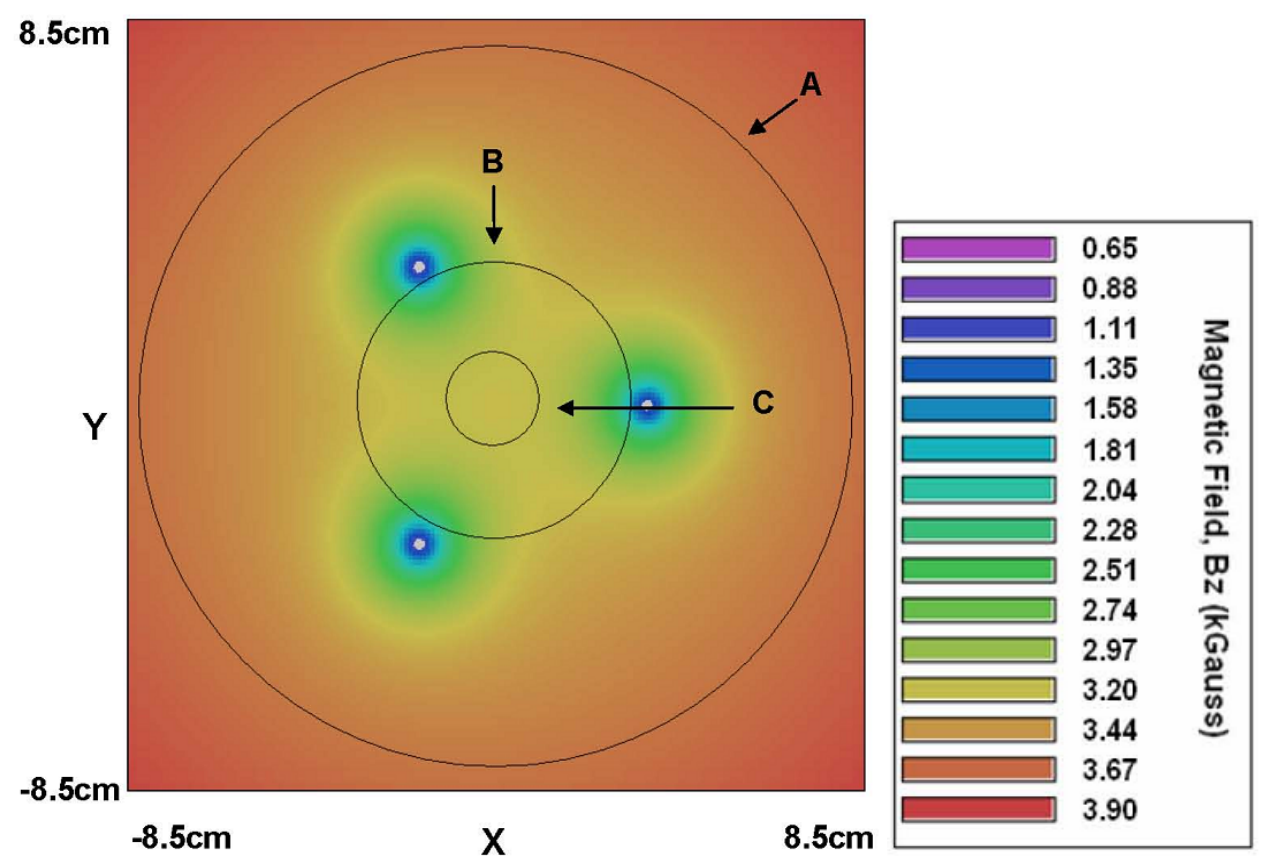

Figure 19. Contour plot of the axial magnetic field for the case of magnetic priming at the anode. Wire length $=4 \mathrm{~cm}$. Wire relative magnetic permeability $=1000 \mathrm{~A}$ : Anode back wall (radius $=8.3 \mathrm{~cm}$ ), B: Anode block inner boundary (radius $=3.2 \mathrm{~cm}$ ), C: Cathode boundary (radius $=3.2 \mathrm{~cm}$ ), C: Cathode boundary (radius $=1.1 \mathrm{~cm})$.

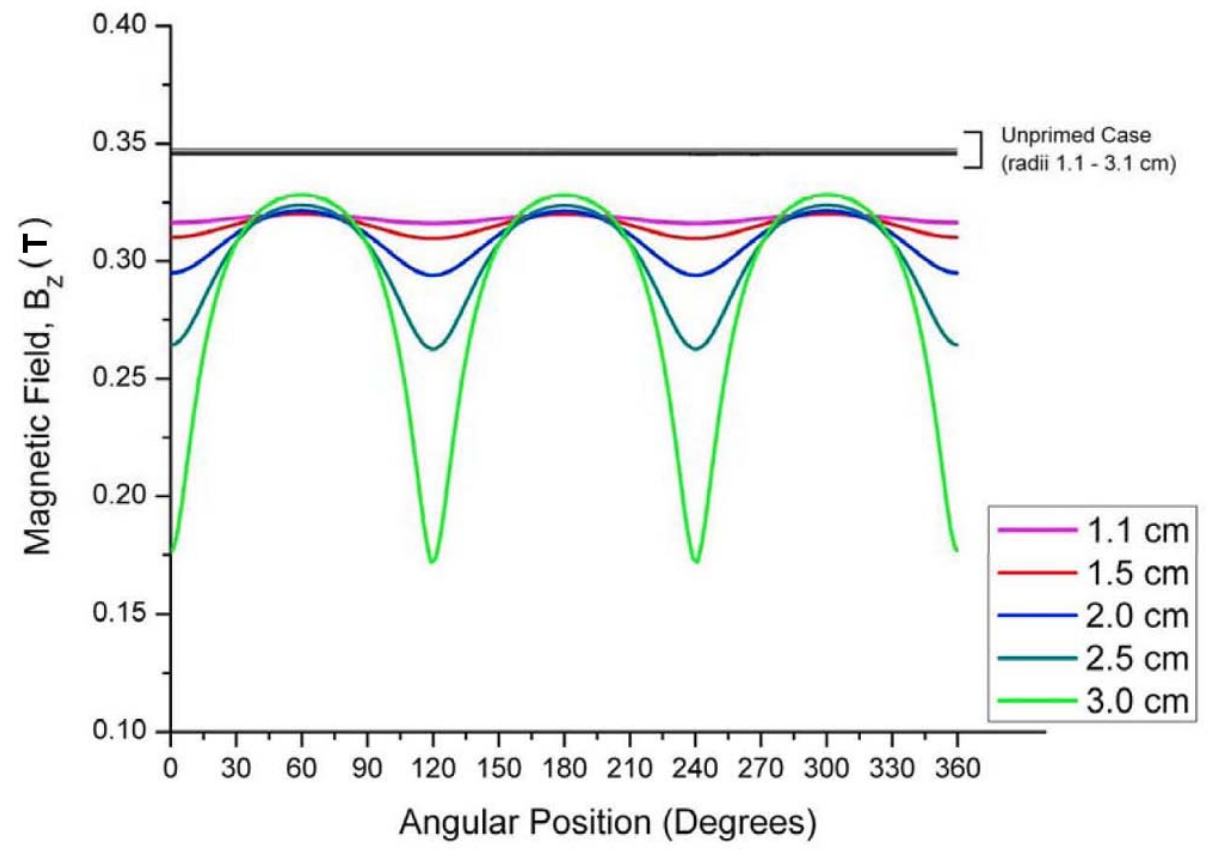

Figure 20. Axial magnetic field, $B_{z}$, as a function of angle at radii $1.1 \mathrm{~cm}-3.1 \mathrm{~cm}$ (cathode radius $=1.1 \mathrm{~cm}$, wire $\mu_{\mathrm{r}}=1000$ ) for the case of magnetic priming at the anode. Unprimed (baseline) case shown for comparison. 
The combined magnetic priming case utilized anode-embedded wires, which were aligned azimuthally with the cathode wires. The results for this case showed strong magnetic priming at the cathode surface, which decreased in amplitude toward the center of the interaction region then intensified in close proximity of the anode block. Figure 21 and Figure 22 detail the results from the case of combined magnetic priming of both the cathode and the anode. As with the magnetic priming of the anode case, the combined priming case showed an overall suppression of the axial magnetic field due to the presence of the wires.

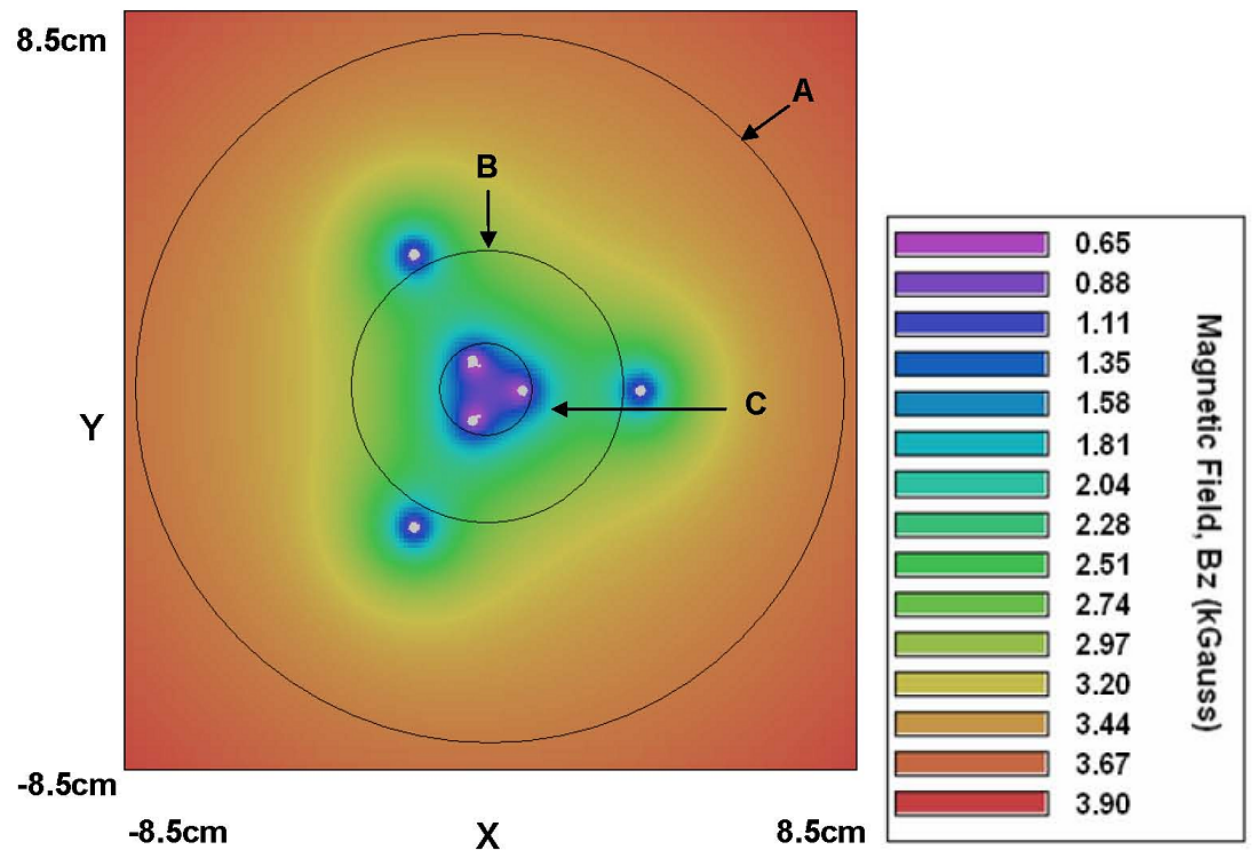

Figure 21. Contour plot of the axial magnetic field for the case of combined magnetic priming at the cathode and the anode. Wire length $=4 \mathrm{~cm}$. Wire relative magnetic permeability $=1000$; Radial locations: A: Anode backwall (radius $=8.3 \mathrm{~cm}$ ), B: Anode block inner boundary (radius $=3.2 \mathrm{~cm}$ ), C: Cathode boundary (radius $=1.1 \mathrm{~cm}$ ). 


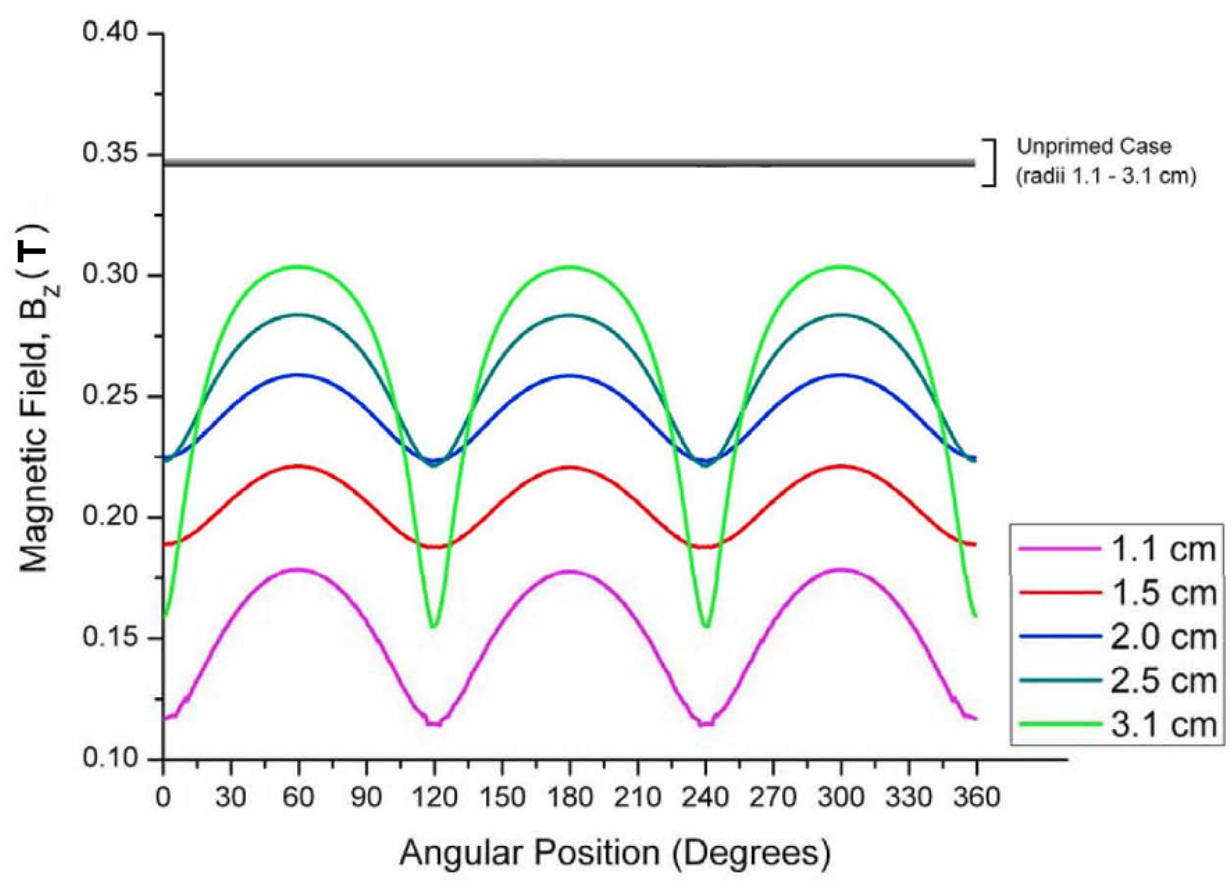

Figure 22. Axial Magnetic Field as a Function of Angle at Radii $1.1 \mathrm{~cm}-3.1 \mathrm{~cm}$ (Cathode Radius $=1.1 \mathrm{~cm}$, wire $\mu_{\mathrm{r}}=1000$ ) for the case of combined magnetic priming at the cathode and anode. Unprimed (baseline) case shown for comparison.

In addition to the perturbations imposed on the global Z-oriented magnetic field, it is expected that, due to edge effects of the finite wire lengths, radial and $\theta$-directed magnetic perturbations are introduced (per $\nabla \cdot B=0$ and $\nabla \times B=0)$. Enhancements to the radial and $\theta$ directed components of the magnetic field were not expected to be of great concern in cases where the magnetic priming structures are of approximately the same size as the magnetron interaction region, such as in the work done by Neculaes et al. [10-13,19] and Luginsland et al. [21], or in cases where they were ignored completely, as with the 2-D simulations performed by Jones et al. [16-18]. Because the magnetic priming wire lengths proposed for use in the relativistic magnetron can be as small as $15 \%$ of the anode length, these edge effects are more important. 
To illustrate the potential importance of the perturbation effects caused by the ends of the wires, the case of three, $6 \mathrm{~cm}$ long wires with a relative permeability of 500 is presented. Figure 23, previously shown as Figure 2 (b), depicts a representation of the UM/L-3 relativistic magnetron cathode, created with the Field Precision Magnum software. This image provides a reference as to the approximate location of the wires within the cathode. The $Z$ axis oriented line passing through $X=Y=0$ is the center axis of both the cathode (shown) and the magnetron (not shown). Each wire is centered lengthwise at $Z=0$. Additionally for this discussion, the radial direction is defined as perpendicular to the surface of the cathode and perpendicular to the $Z$ axis. The $\theta$ direction is defined as parallel to the circumference of the cathode at a given point and perpendicular to the $Z$ axis.

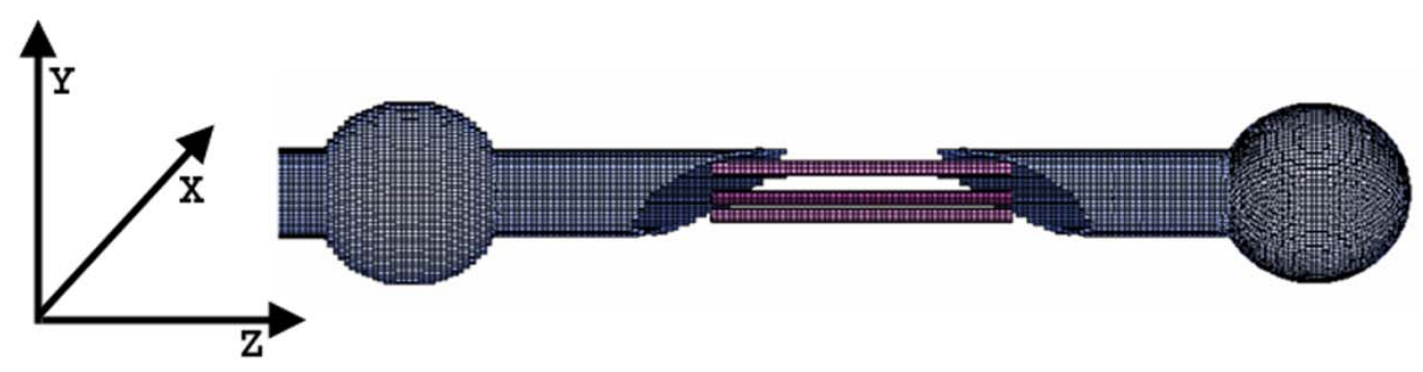

Figure 23. Close-up cutaway view of the cathode emission region showing wire locations.

Figure 24 (a) depicts the location of a contour map of the magnitude of the magnetic field, sampled across the $X-Z$ plane at which the $Y$ coordinate is equal to zero. This plane intersects the center axis of both the cathode and the simulation space and bisects one of the three priming wires. In Figure 24 (b) the contour map of the magnitude of B is overlaid with an $X-Z$ vector field plot, 
showing the orientation of the magnetic field at each vector location. At the center of the cathode, where $Z$ is equal to zero, the magnetic field is aligned almost completely with the $Z$ axis of the simulation; however, toward the ends of the wire, the magnetic field vector plot shows a very strong radial orientation close to the cathode surface.

In order to more effectively compare the relative magnitude of the magnetic field components, $X-Y$ plane contour slices of the $B$ field magnitude are taken at $Z=0 \mathrm{~cm}$ and $Z=2.6 \mathrm{~cm}$, as shown in Figure $25(\mathrm{a})$ and Figure $25(\mathrm{~b})$ respectively. X-Y oriented vector plots are then overlaid in a similar manner as was done with Figure 24 (b), yielding the plots displayed in Figure 26 (a) and (b). In these plots, the cathode boundary is represented by the black circle. Both the $Z=0$ plane (Figure $26(\mathrm{a})$ ) and the $Z=-2.6 \mathrm{~cm}$ plane (Figure $26(\mathrm{~b})$ ) show evidence of magnetic field components oriented in both the radial and $\theta$ directions. 


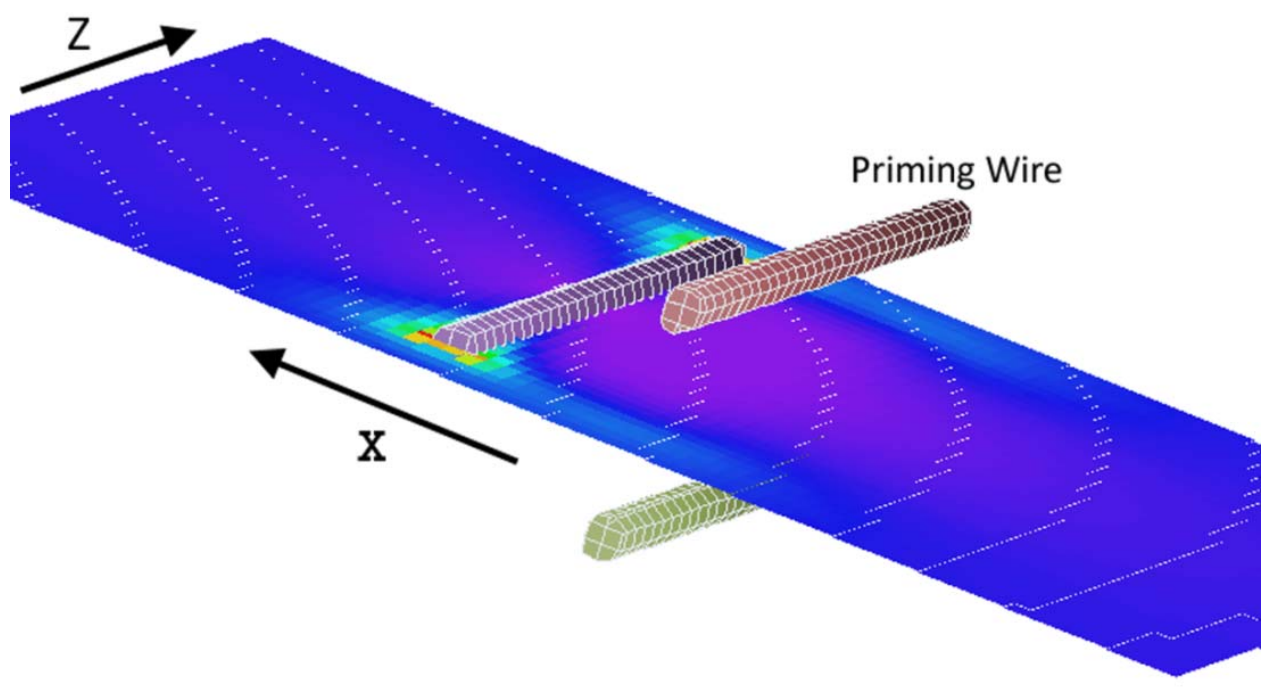

(a)

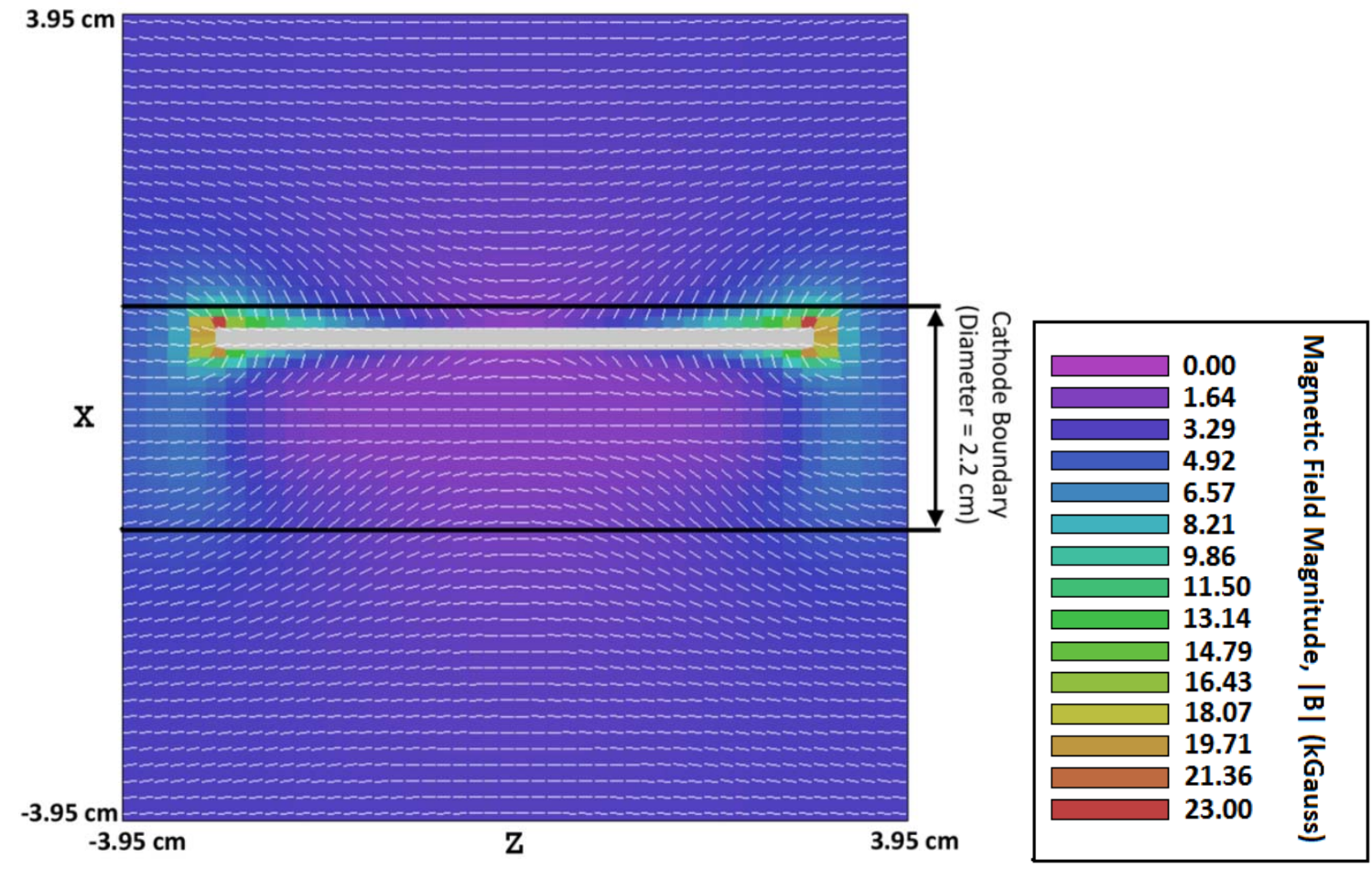

(b)

Figure 24. (a) Contour map of $|\mathrm{B}|$, showing relation to the magnetic priming wires. (b) Contour map of $|B|$ with $X-Z$ magnetic field vector overlay (priming wire shown in gray). The strong radial component of the $B$ field at the lower boundary of the cathode is due to the combined effects of the two neighboring wires outside the contour slice. 


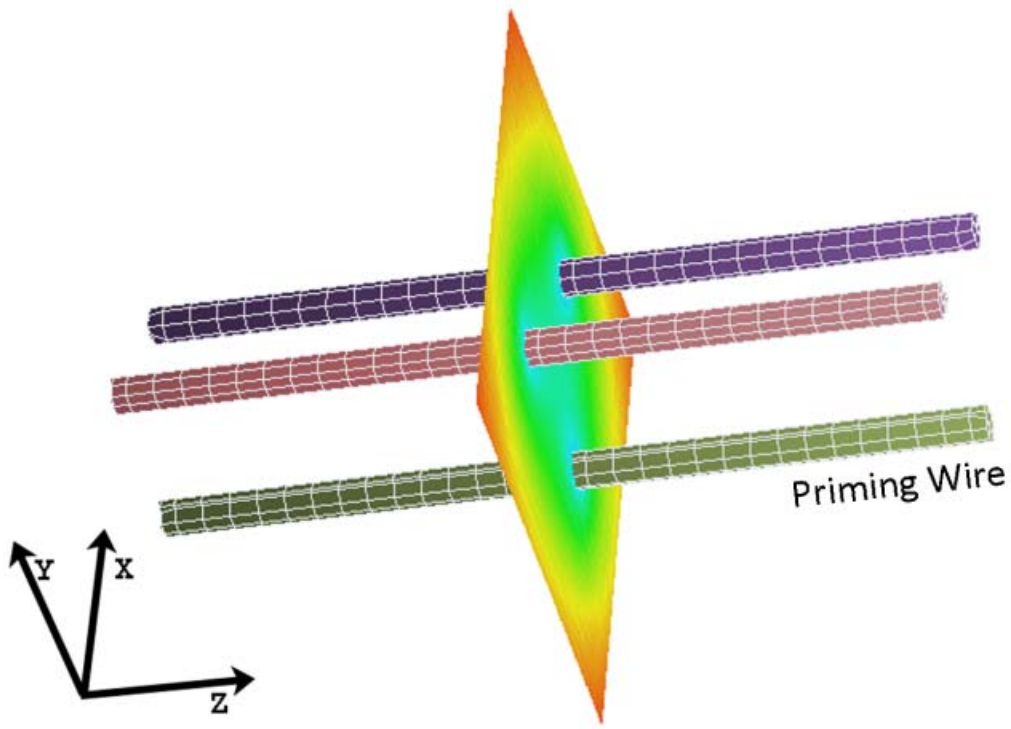

(a)

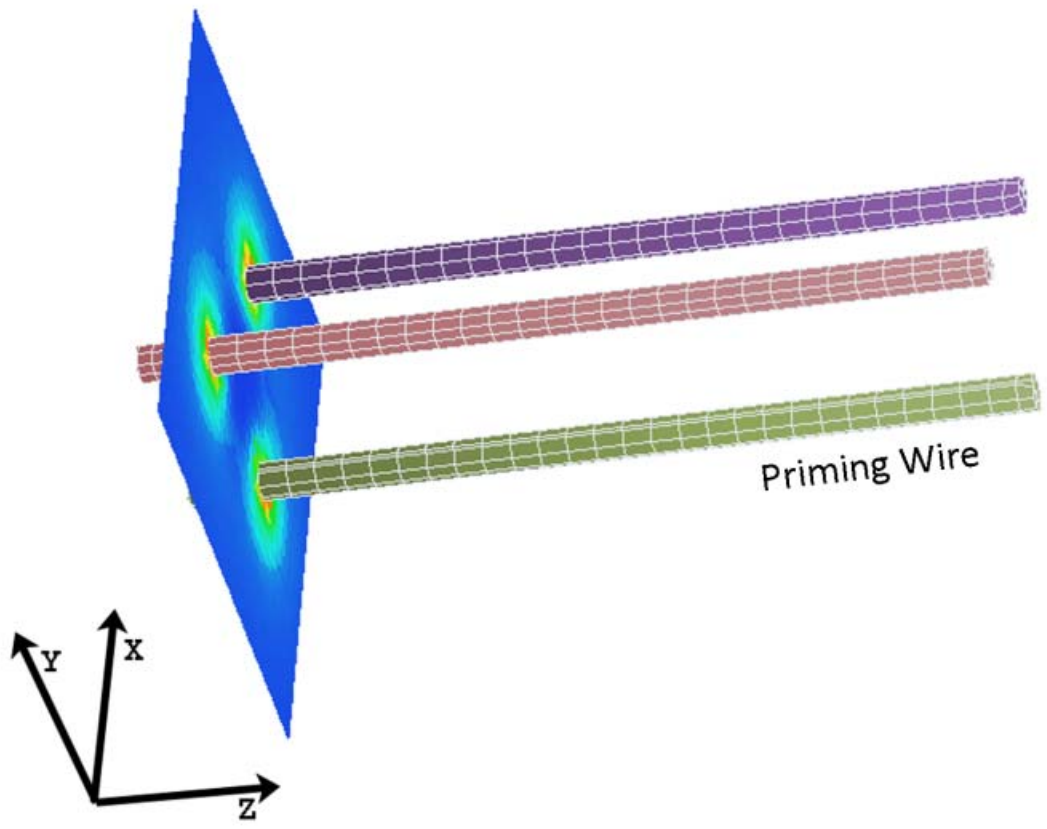

(b)

Figure 25. $|B|$ contour plane references $(a) Z=0 \mathrm{~cm}(\mathrm{~b}) \mathrm{Z}=-2.6 \mathrm{~cm}$ 

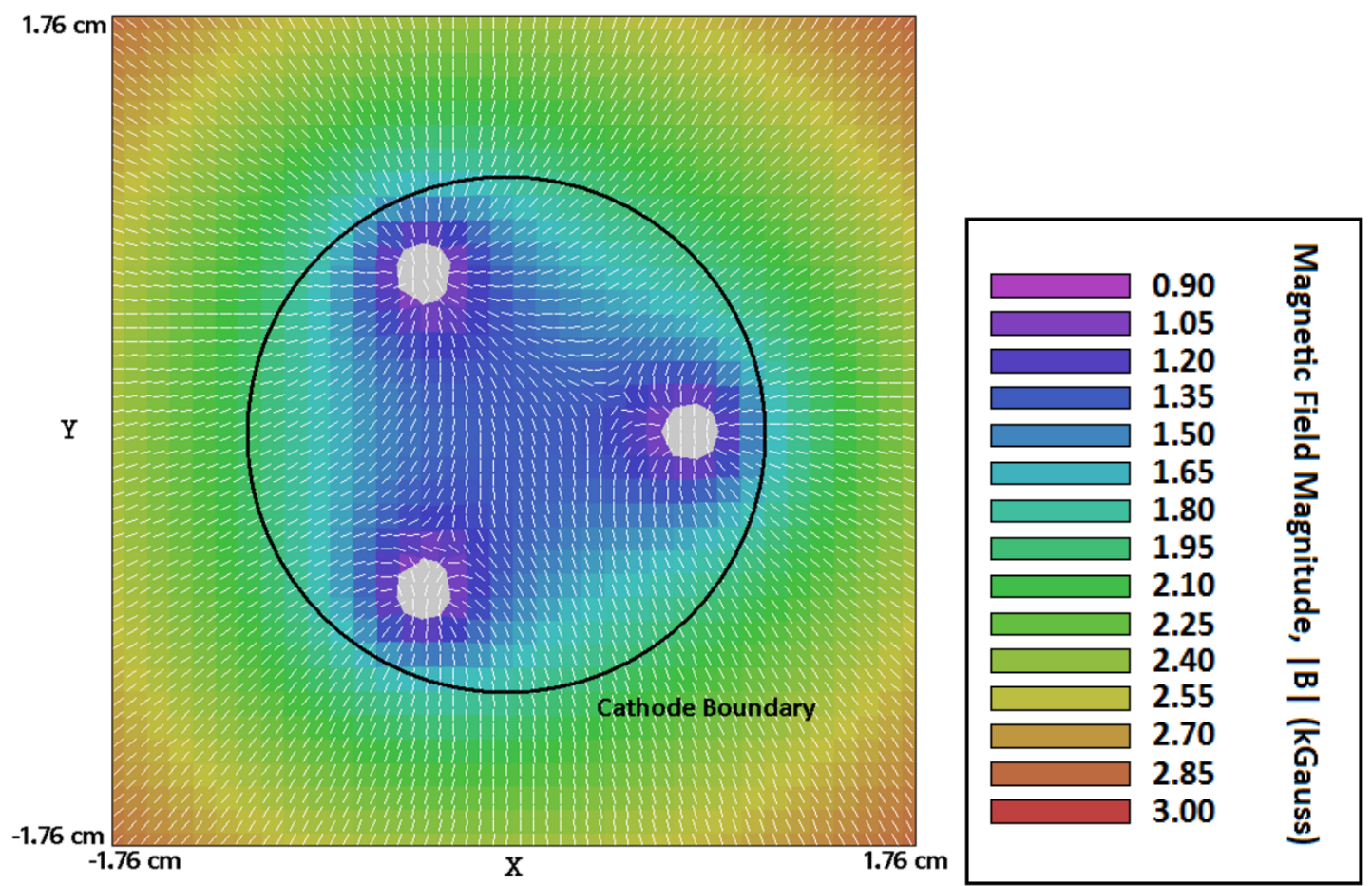

(a)
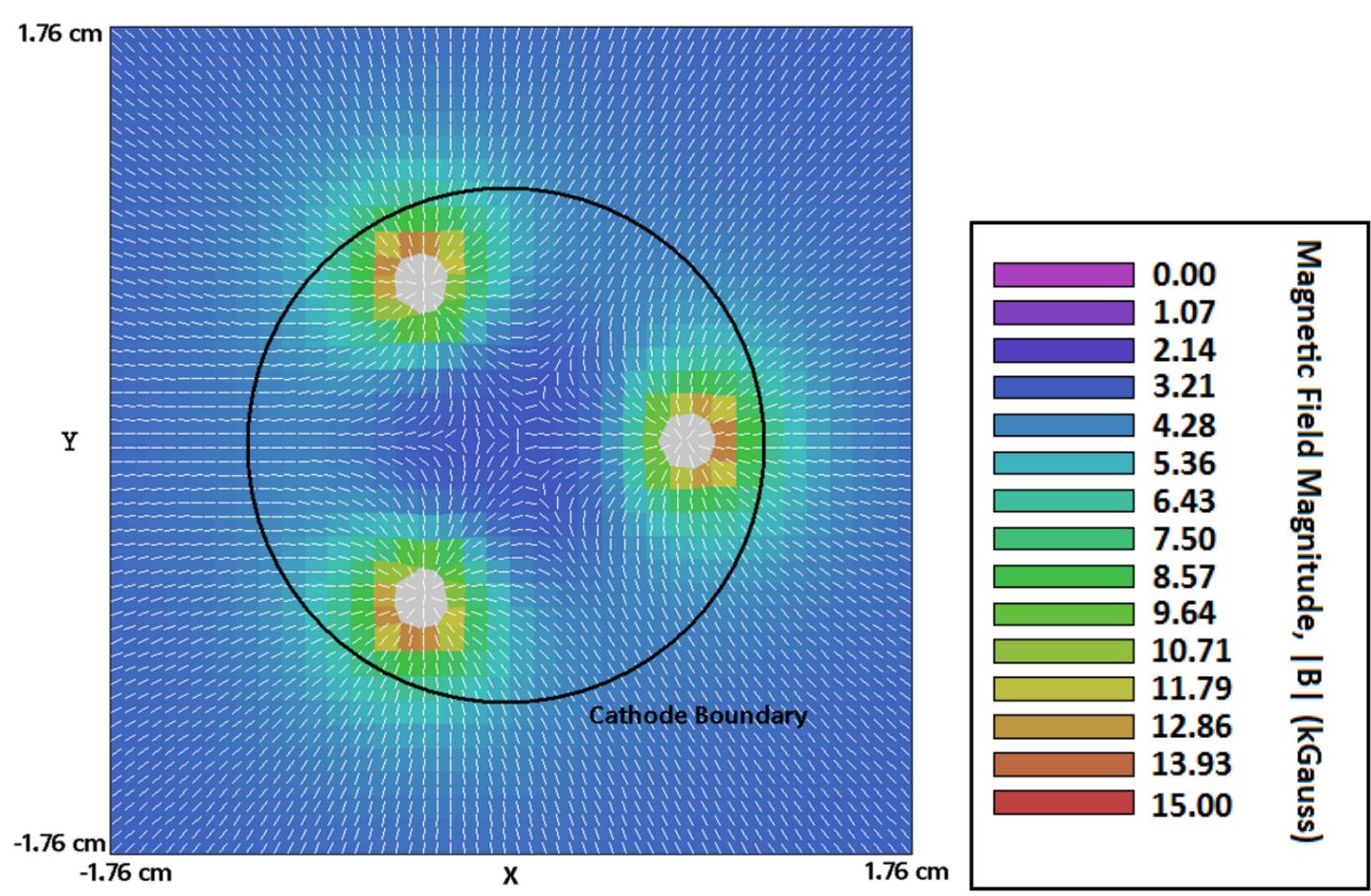

(b)

Figure 26. Contour map of $|B|$ with $X-Y$ magnetic field vector overlay (priming wire shown in gray), where (a) $Z=0 \mathrm{~cm}$ and (b) $Z=-2.6 \mathrm{~cm}$. 
Figure $27(\mathrm{a}),(\mathrm{b})$, and (c) are plots of the radial, $\theta$, and $Z$ components of the magnetic field at points in the $Z=0$ plane at various distances away from the cathode centerline. The cathode boundary is located at a radius of $1.1 \mathrm{~cm}$. As is evident from the three plots, in the $Z=0 \mathrm{~cm}$ plane, the amplitudes of the $B_{r}$ and $B_{\theta}$ perturbations are vanishingly small, compared to the magnitude of the perturbations in $B_{Z}$. This is in stark contrast to the plots of the radial, $\theta$, and $Z$ components of the magnetic field at points in the $Z=-2.6 \mathrm{~cm}$ plane, displayed in Figure $28(a)$ - (c). Even though the amplitude of the $B_{z}$ perturbations increased by a factor of two (up to $1 \mathrm{kGauss}$ ) with respect to the $Z=0 \mathrm{~cm}$ plane plots, the $B_{\theta}$ and $B_{r}$ perturbations are still many times greater in amplitude (up to 6 kGauss). 


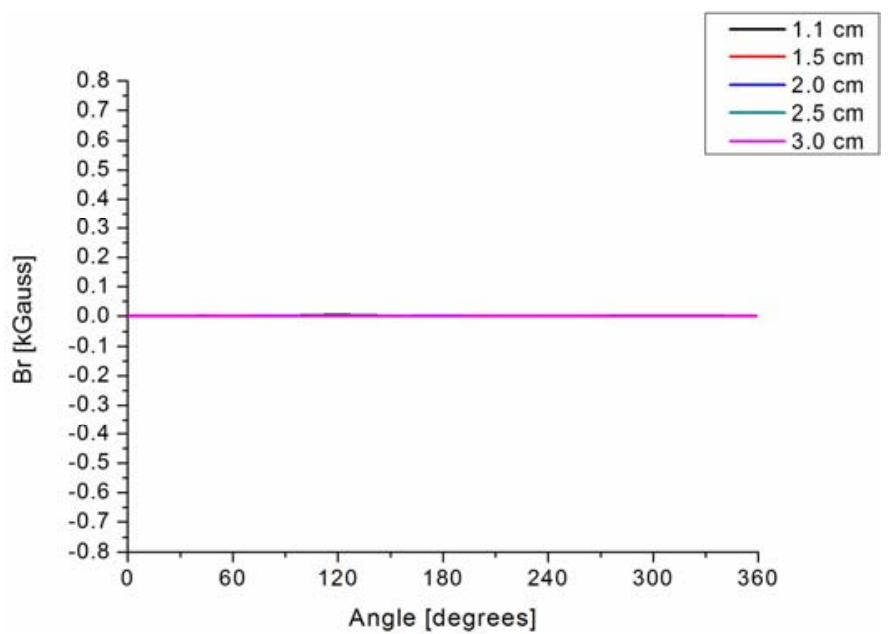

(a)

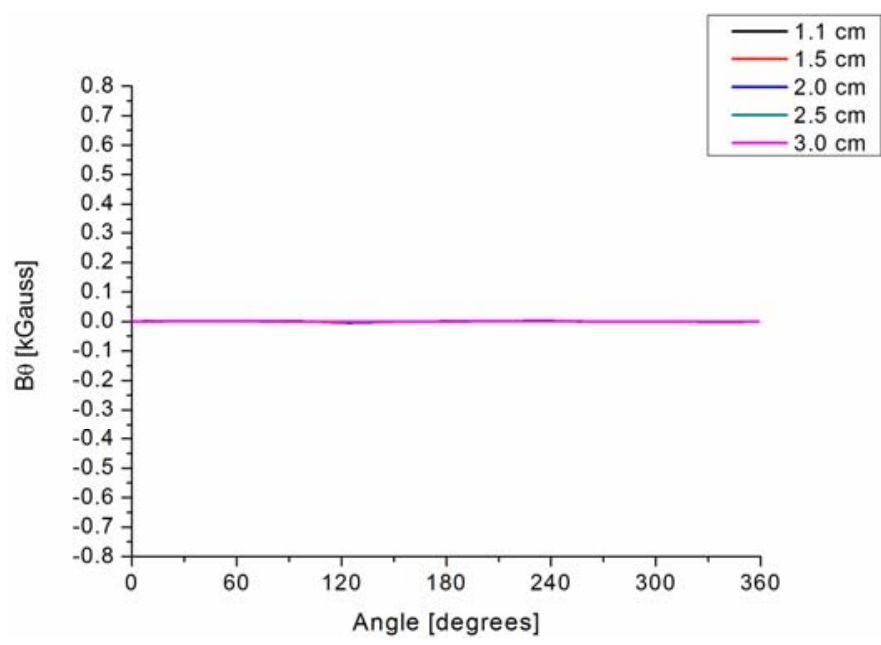

(b)

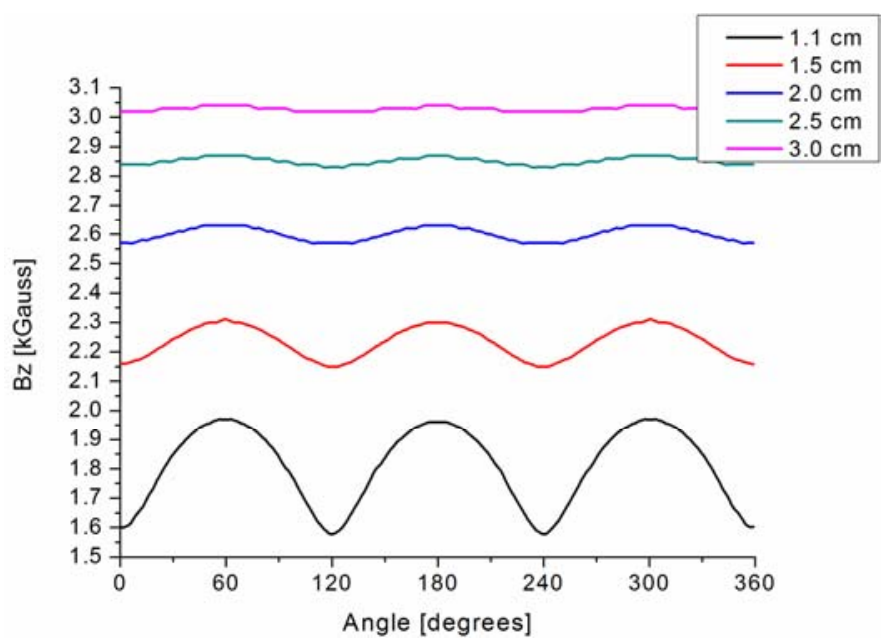

(c)

Figure 27. Magnetic field component plots for the $Z=0$ plane at various radii (a) $B_{r},(b) B_{\theta}$, (c) $B_{Z}$. 


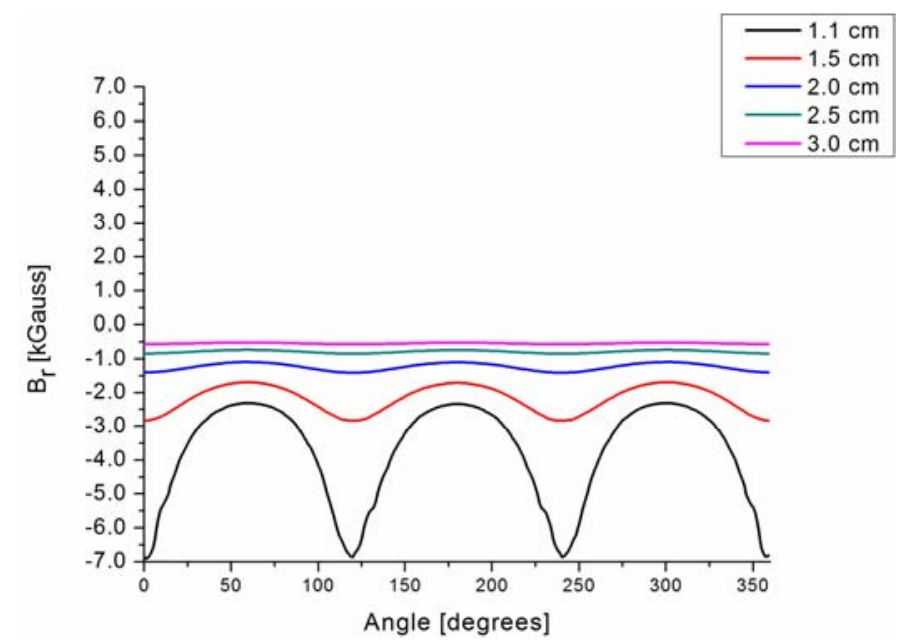

(a)

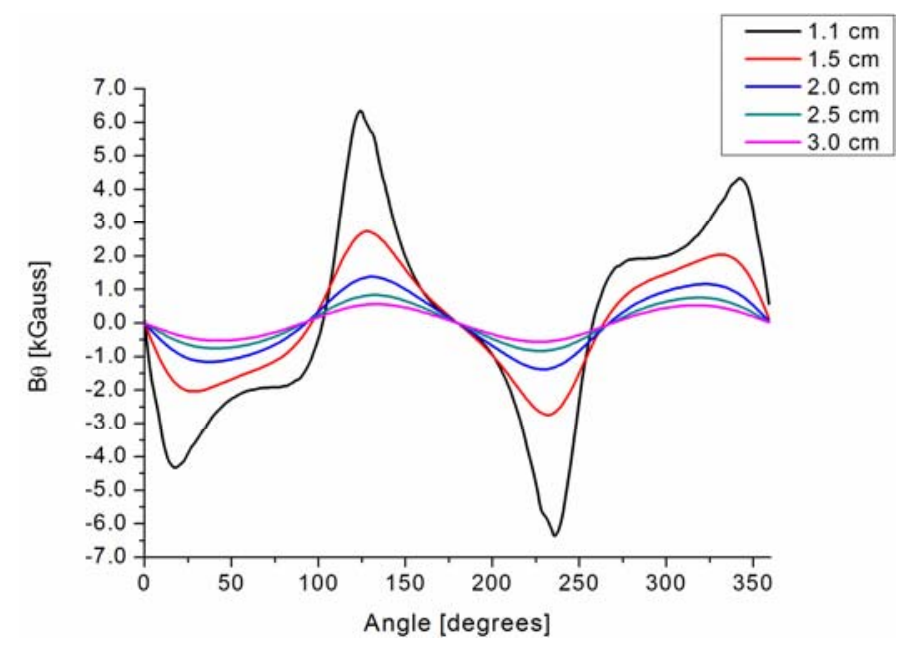

(b)

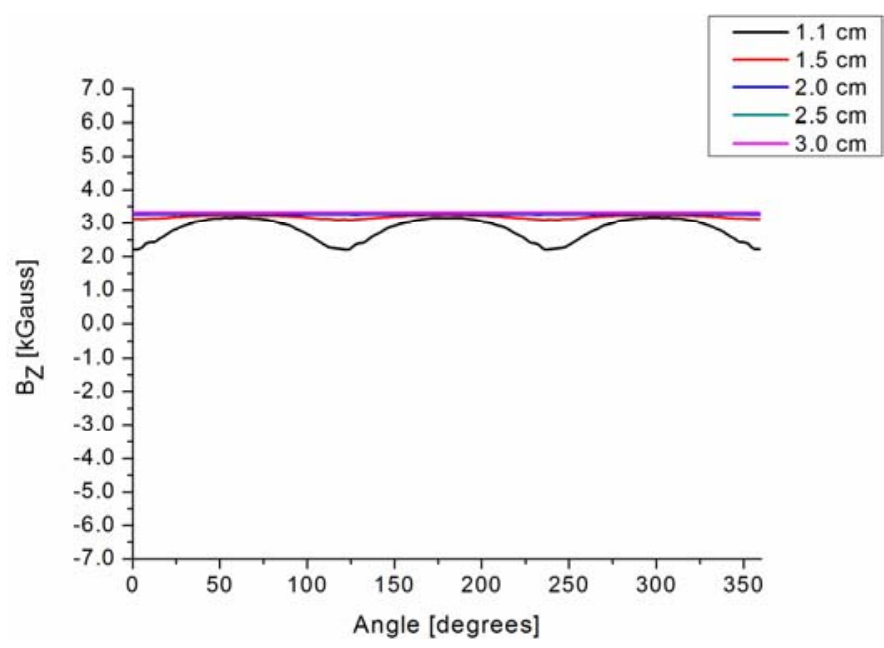

(c)

Figure 28. Magnetic field component plots for the $Z=-2.6 \mathrm{~cm}$ plane at various radii (a) $B_{r}$, (b) $B_{\theta}$, (c) $B_{Z}$. 


\subsection{Electromagnetic Particle-in-Cell Simulations}

Magnetic field data obtained from the magnetostatics simulations were imported into 3-D MAGIC code and run for the case of a relativistic A-6 magnetron (all three magnetic field components were imported, thus ensuring $\nabla \cdot B=0)$. To reduce simulation run time, a number of simplifications have been made in the model magnetron from the true experimental setup. Cathode voltage was ramped to negative $300 \mathrm{kV}$, which corresponds to the average experimental cathode voltage. The risetime of the ramped voltage signal is approximately one RF period, $\sim 1 \mathrm{~ns}$ (vs. $\sim 125 \mathrm{~ns}$ in the experiment). Anode vanes are conformal (not rounded) in the simulation. Cathode diameter was set at $2.2 \mathrm{~cm}$ (compared to the experimental cathode diameter of $1.27 \mathrm{~cm}$ ). The larger cathode (compared to the experiment) in the simulation allowed for the reduction of the simulation computation time to a reasonable length $(\sim 1$ week versus multiple weeks) Simulation geometry is detailed in Figure 29. 


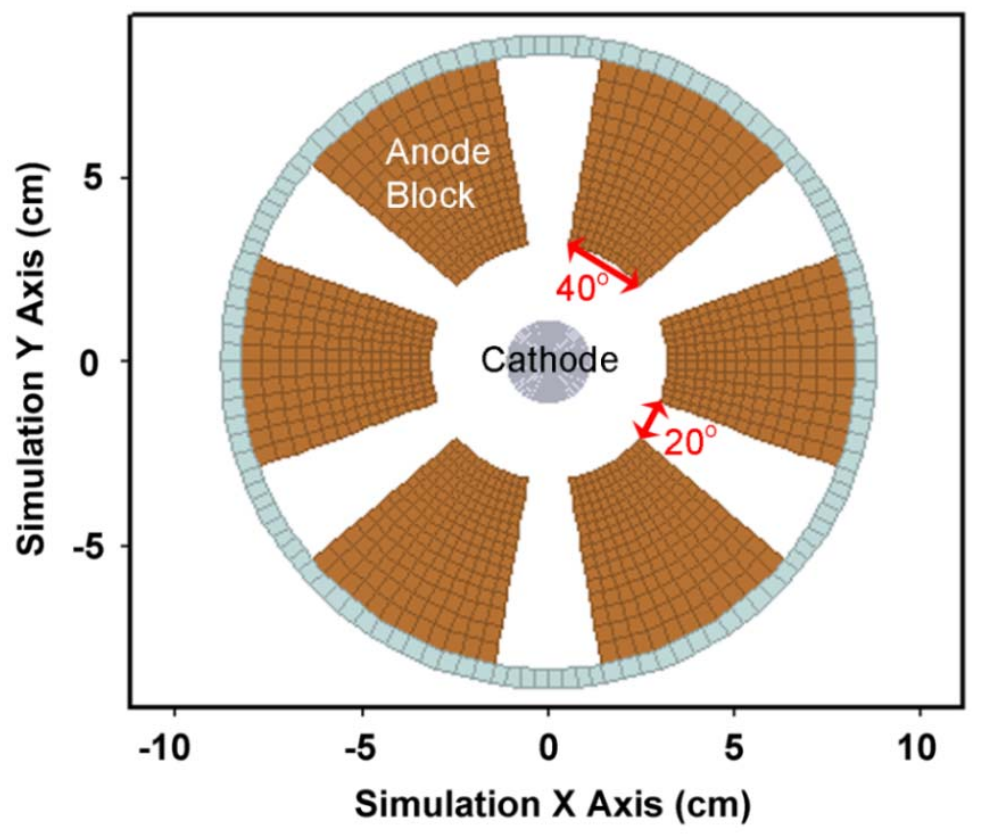

(a)

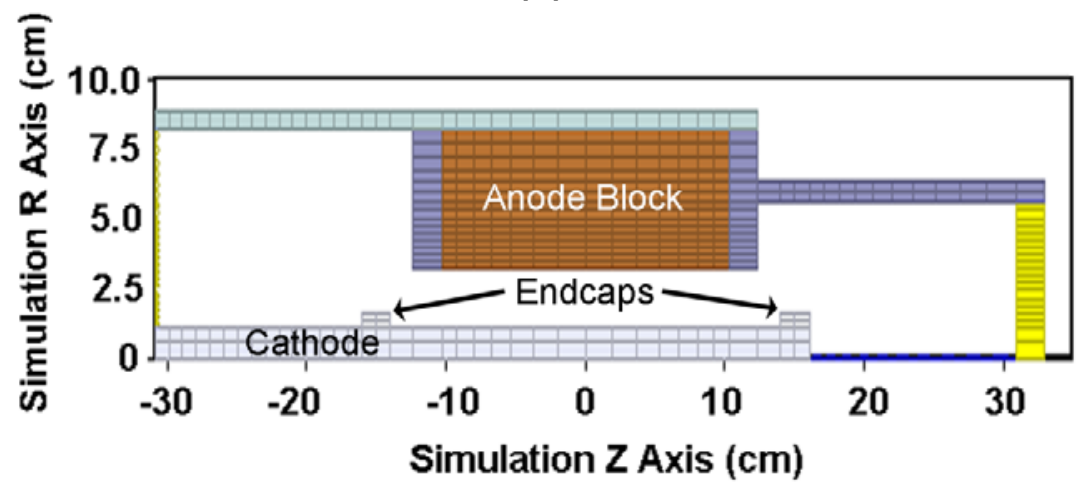

(b)

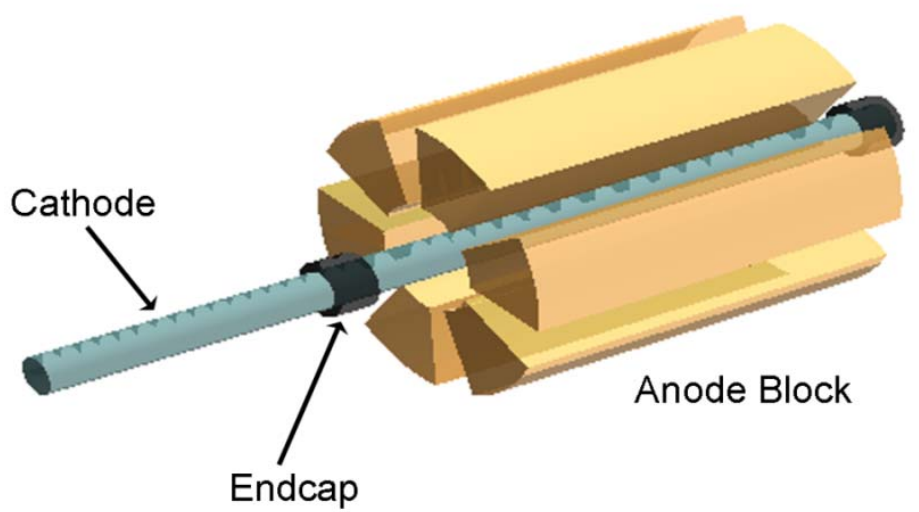

(c)

Figure 29. MAGIC simulation geometry. (a) Radial cross-section of simulation magnetron. (b) Axial cross-section of simulation magnetron. (c) 3-D view of simulation magnetron (only cathode and anode vanes are visible, other components not shown). 
The degree of success of each simulated magnetic priming case studied was based on start-oscillation time and power. In the following simulations, the peak electric field magnitude measured between anode vanes is used as a surrogate for power as the electric field squared should be proportional to the power output from a given resonator cavity. Investigating degree of mode control (i.e. ability to enhance the probability of $\pi$-mode start up over other unwanted modes), which was previously demonstrated by Jones et. al. in 2-D relativistic magnetrons with the idealized magnetic priming fields, would have required many times more simulation runs and longer computational times. Thus, the effect of magnetic priming on mode control was not investigated in the simulation research done on this project.

The initial primed case simulated in MAGIC was magnetic priming at the cathode using sets of the priming wires with relative permeability values of 1000 , shown in Figure 30. Although the primed cases started up faster than the unprimed case, they performed poorly due to excessive end loss currents. Figure 31 depicts an example of a priming case exhibiting excessive endloss. The case shown in Figure 31 was primed using $16 \mathrm{~cm}$ long wires with a relative permeability of 1000 . The priming amplitudes created by the $\mu_{r}=500$ wires yielded milder perturbations (and reduced end loss) (approximately $60 \%$ of the $\mu_{\mathrm{r}}$ $=1000$ wires) and were used in all subsequent PIC simulations. 

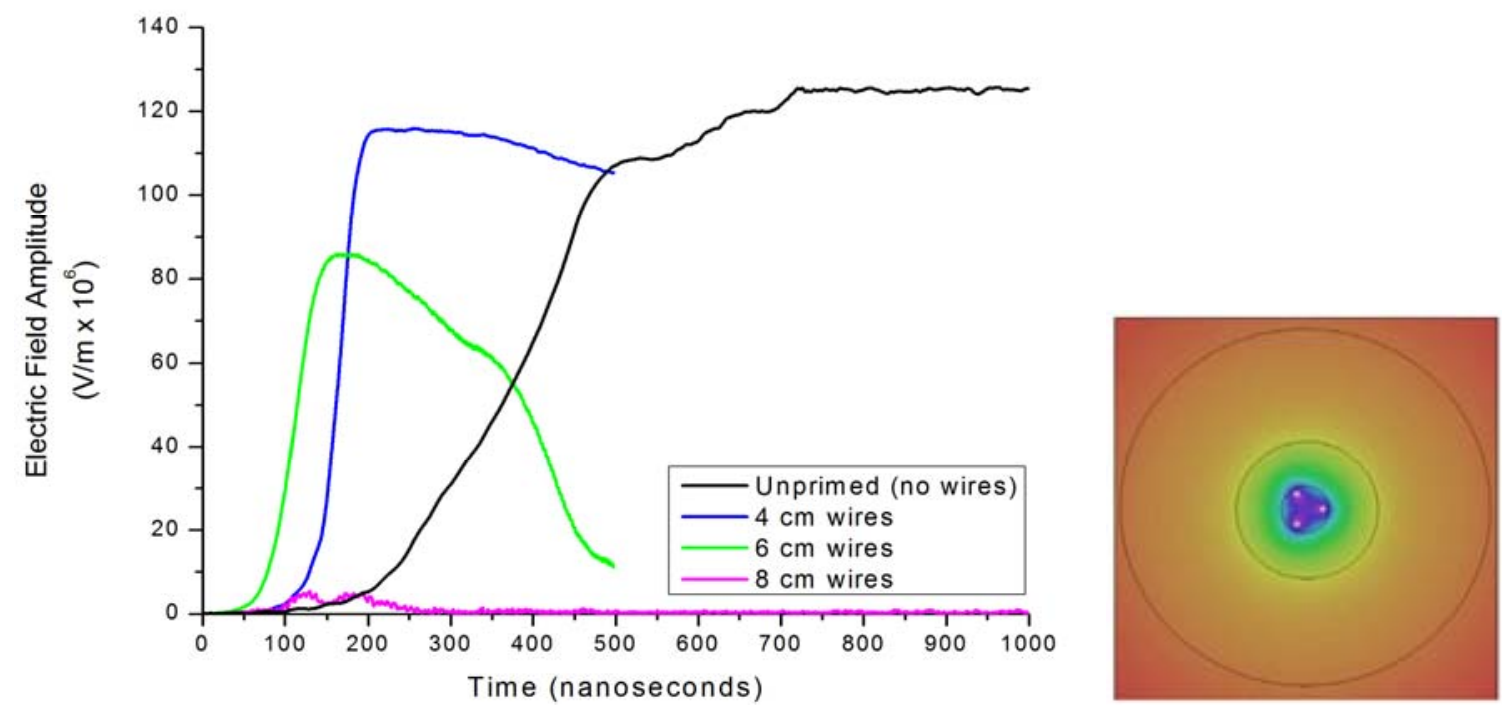

Figure 30. Magnetron RF electric field amplitude as a function of time for the case of magnetic priming at the cathode. (Wire $\mu_{r}=1000$ ). The magnetic contour plot to the right of the graph is shown as a visual reference of imposed field perturbations.

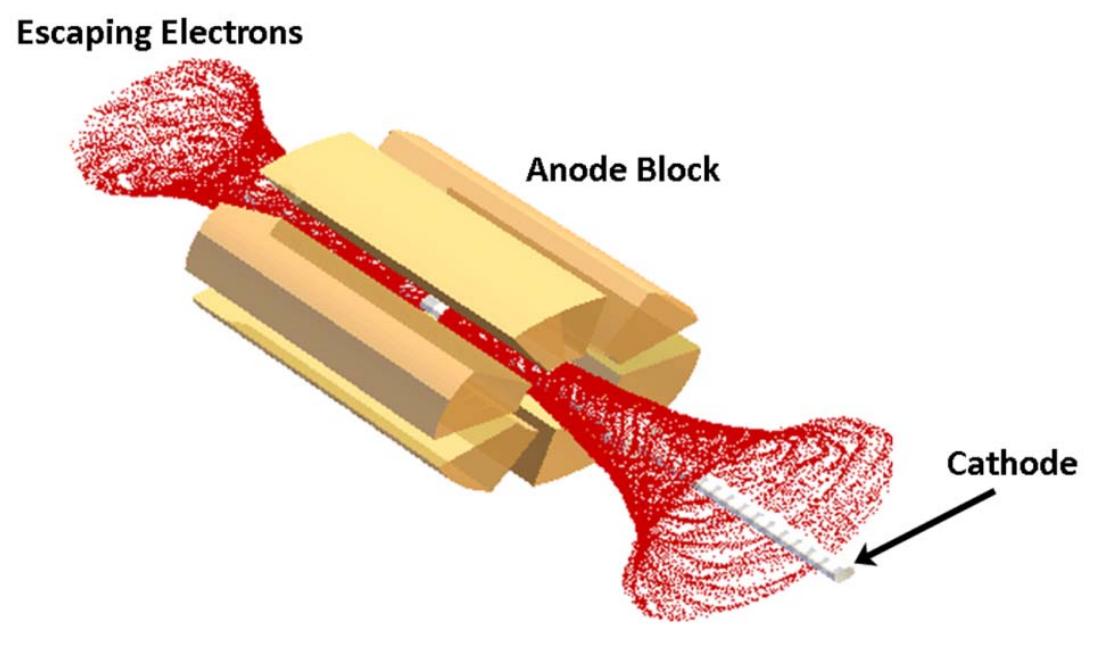

Figure 31. Image from a MAGIC PIC simulation illustrating excessive electron endloss.

Figure 32 displays data from simulations of magnetic priming at the cathode using wires with relative magnetic permeability values of 500 . This priming scheme showed improvements in microwave start-oscillation time and 
comparable power output to the unprimed (baseline) case for a range of wire lengths from 3 to $6 \mathrm{~cm}$. As wires were extended beyond the optimal length of 4 $\mathrm{cm}$, magnetron operation became unstable, resulting in reduced output power and, eventually, failure to oscillate. In both the $\mu_{r}=500$ and $\mu_{r}=1000$ cathode magnetic priming simulations, optimal priming conditions were achieved using four centimeter wires.

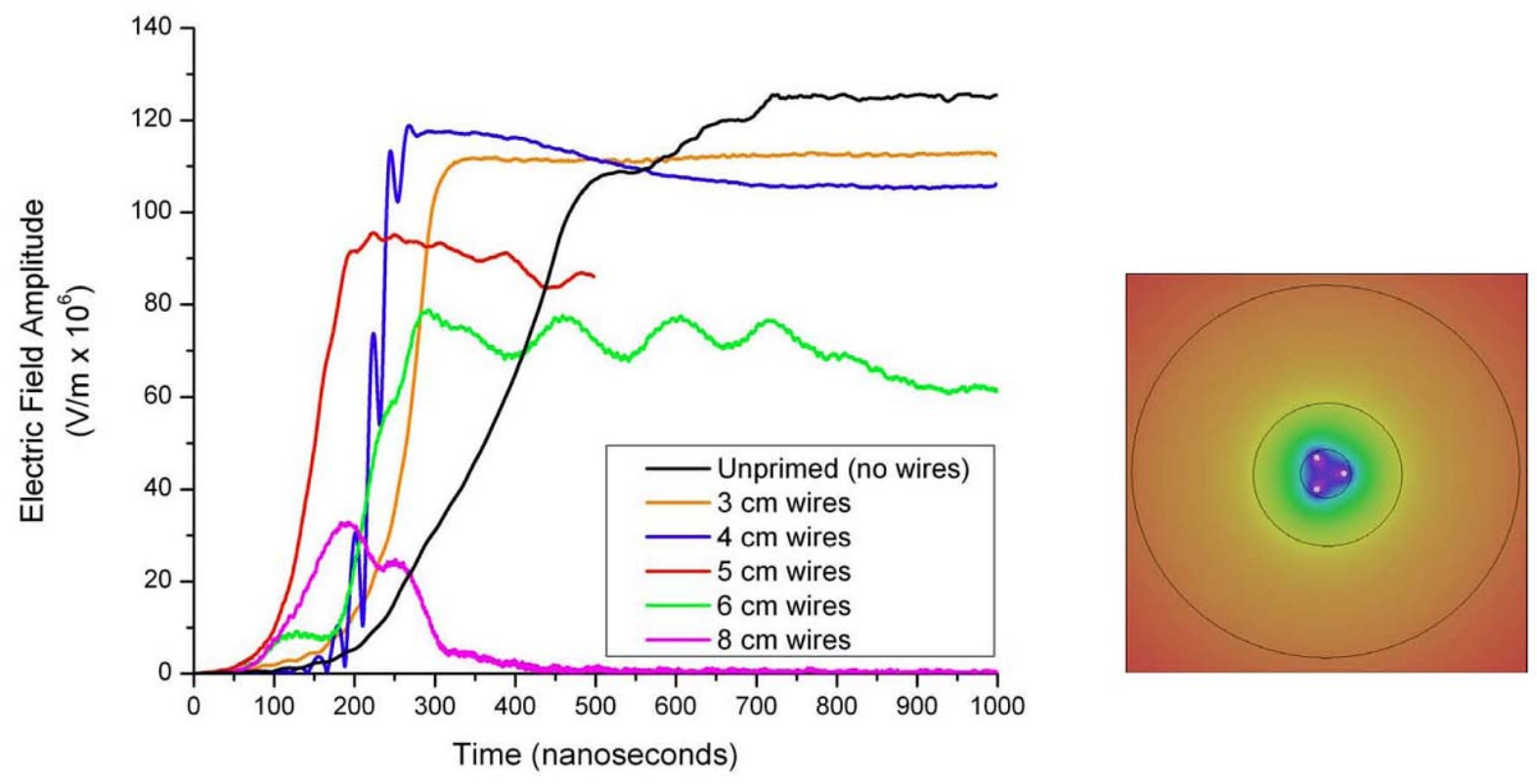

Figure 32. Magnetron RF electric field amplitude as a function of time for the case of magnetic priming at the cathode. (Wire $\mu_{r}=500$ ). The magnetic contour plot to the right of the graph is shown as a visual reference of imposed field perturbations. A small degree of mode competition was observed in the $4 \mathrm{~cm}$ wire case, as evidenced by the beat pattern imposed on the power trace.

Simulations of magnetic priming only at the anode were performed for wires with a relative magnetic permeability of 500 . As expected, results from these simulations were less promising than for the case of magnetic priming at the cathode. For shorter wire lengths $(4 \mathrm{~cm}$ and $6 \mathrm{~cm})$ the magnetron showed 
lower power operation with no positive effect on start-oscillation time. In the $8 \mathrm{~cm}$ and $10 \mathrm{~cm}$ wire cases, the simulation magnetron showed faster startup, but still operated with a much lower power output than the unprimed baseline case. It is possible that while the perturbations created in the $8 \mathrm{~cm}$ and $10 \mathrm{~cm}$ wire cases were strong enough to initiate faster startup, they were also strong enough to alter the synchronism condition of the electrons near the anode, thus reducing the amount of energy these electrons contributed to the RF wave. Simulations results for magnetic priming of the anode are detailed in Figure 33.
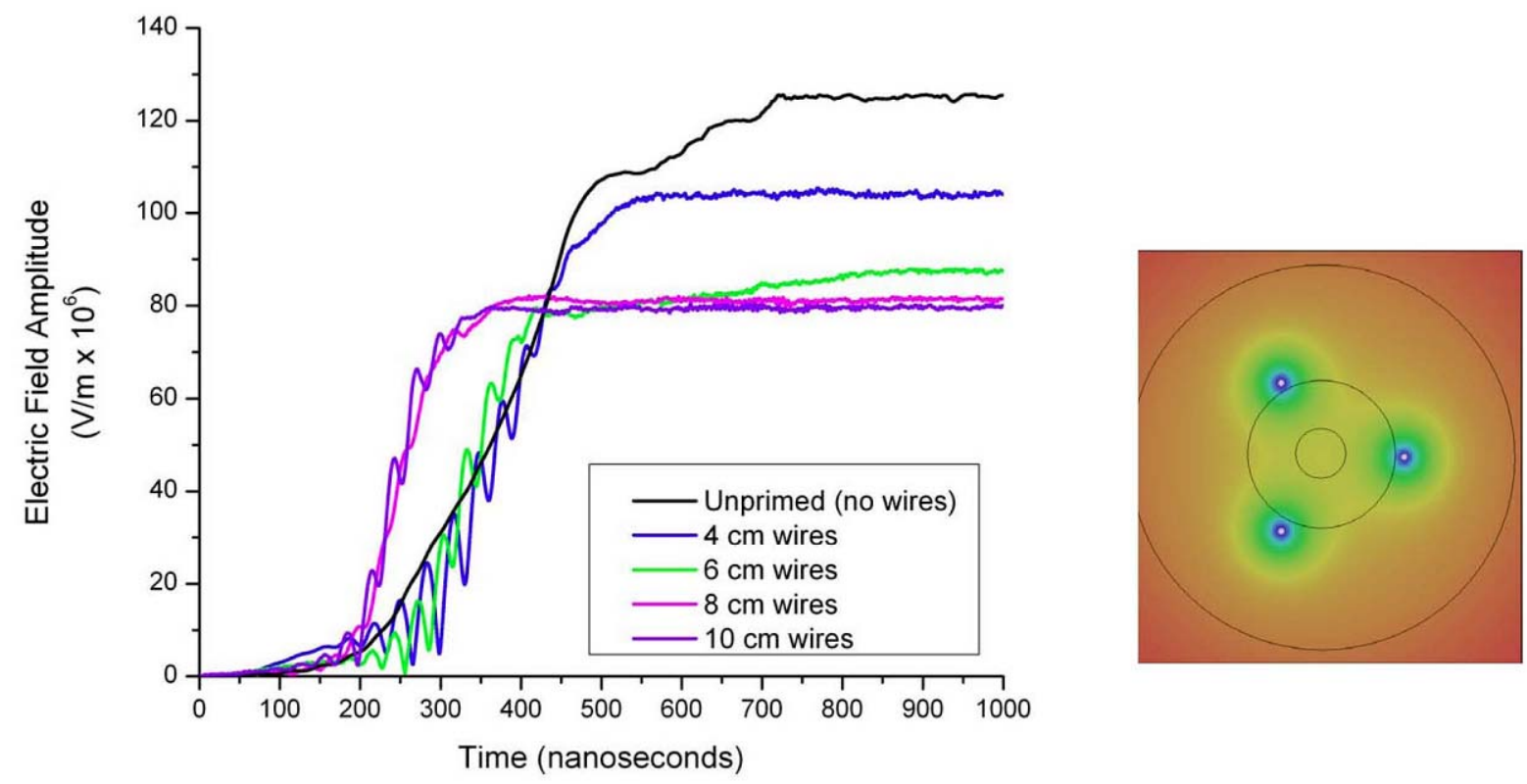

Figure 33. Magnetron RF electric field amplitude as a function of time for the case of magnetic priming at the anode. (Wire $\mathrm{mu}=500$ ). The magnetic contour plot to the right of the graph is shown as a visual reference of imposed field perturbations.

Finally, Figure 34 shows comparisons between: a) combined magnetic priming at both the cathode and anode, versus $b$ ) magnetic priming at the cathode, and c) unprimed case. For the combined anode-cathode magnetic 
priming simulation, the fastest magnetron start-oscillation was achieved using four centimeter wires in the cathode and four centimeter wires in the anode. While the cathode-only magnetic priming case showed a higher initial power than the combined priming case, equilibrium microwave power outputs from these two conditions were approximately equal.
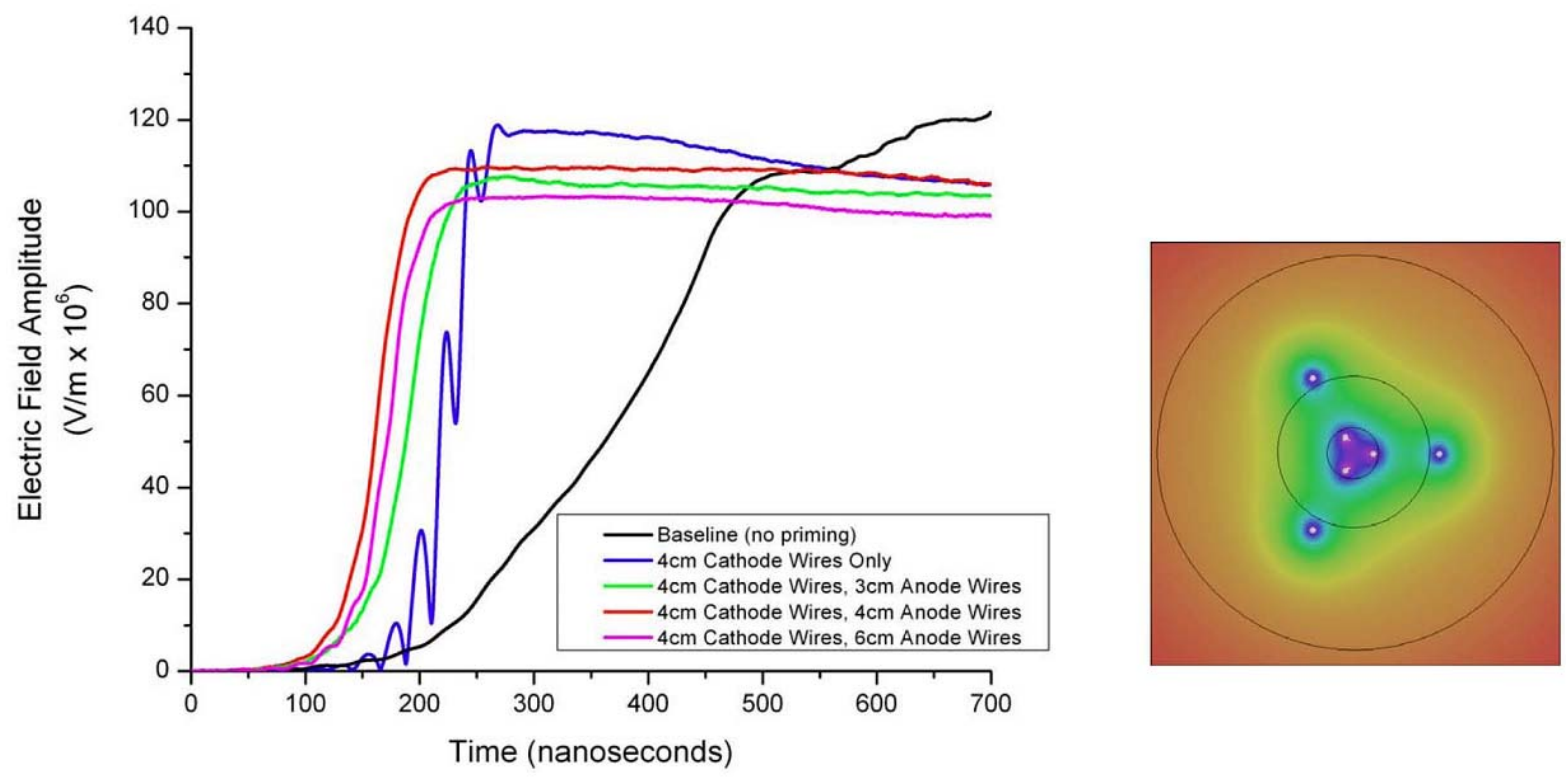

Figure 34. Magnetron RF electric field amplitude as a function of time for the case of combined magnetic priming at the cathode and the anode. (Wire $\mathrm{mu}=500$ ). The magnetic contour plot to the right of the graph is shown as a visual reference of imposed field perturbations.

Simulation results from MAGIC code show dramatic magnetron performance improvements over the baseline for the cases of magnetic priming at the cathode and combined magnetic priming at the cathode and anode. Magnetic priming only at the anode was shown to reduce the overall performance of the simulation magnetron. It was possible to achieve faster startup by magnetic priming at the anode alone, but at the price of substantially 
reduced power output. Experiments have been conducted using magnetic priming at the cathode and at the anode. The experimental results for these cases will be presented in the following chapter. Preliminary results of combined magnetic priming at both the cathode and the anode were inconclusive and are included in Appendix C. 


\section{Chapter 4 \\ Magnetic Priming Experimental Research}

Three magnetic priming experimental campaigns are presented in this dissertation: two utilizing magnetic priming at the cathode and one utilizing magnetic priming at the anode of a relativistic magnetron. Because of the favorable simulation results for the case of three $4 \mathrm{~cm}$ wires in the cathode, the first magnetic priming scheme used in the UM/L-3 Titan relativistic magnetron consisted of three $4 \mathrm{~cm}$ nickel-iron alloy (Hymu 80/Permalloy) wires with diameters of 0.254 centimeters embedded within the cathode. These wires were located directly under the cathode surface, centered under the emission region,

and spaced 120 degrees apart (for N/2 symmetry in a six vane magnetron). Due to unrelated radio frequency priming experiments [31] being performed on the magnetron during the same time frame as the initial magnetic priming experiment, the magnetron was configured in an unbalanced loading condition. This unbalanced configuration consisted of three dissimilar waveguide outputs and terminations, described in further detail in the following section.

The second magnetic priming experiment also utilized nickel-iron alloy wires embedded within the cathode; however, the wires were of slightly smaller diameter $(0.127 \mathrm{~cm})$. In the second magnetic priming experiment, the effects of 
varying the length of the priming wires were explored. Data sets for wire lengths ranging from $4 \mathrm{~cm}$ to $27 \mathrm{~cm}$ were obtained and analyzed. A balanced loading scheme, consisting of three identical waveguides, as well as upgrades to the dielectric window geometry were implemented for the second experiment.

The experiment investigating magnetic priming at the anode of the relativistic magnetron utilized sets of three $0.508 \mathrm{~cm}$ diameter magnetic wires of varying lengths $(10 \mathrm{~cm}$ to $20 \mathrm{~cm})$ installed in alternate anode vanes. The balanced loading configuration and upgraded microwave windows were again used in the third experiment.

\subsection{Experimental Configuration}

The six-vane magnetron and Marx-Abramyan Bank voltage source used for these experiments are the same as those reported by Lopez et al.: the Michigan Electron Long Beam Accelerator-Ceramic insulator (MELBA-C) [53,54]. The UM/L-3 Titan relativistic magnetron is designed with a coupling slot at the back of each anode cavity which allows microwave radiation to directly couple out of any or all of the 6 resonator cavities. In these experiments, three output waveguides were attached to alternate cavities. All other coupling cavities were sealed with vacuum-tight metal plates.

In the first experiment, Lucite windows were used at the vacuum barrier between the magnetron and the waveguides. Figure 35 presents an image of the magnetron housing, output waveguides, magnet coils, and a number of diagnostic connections. 


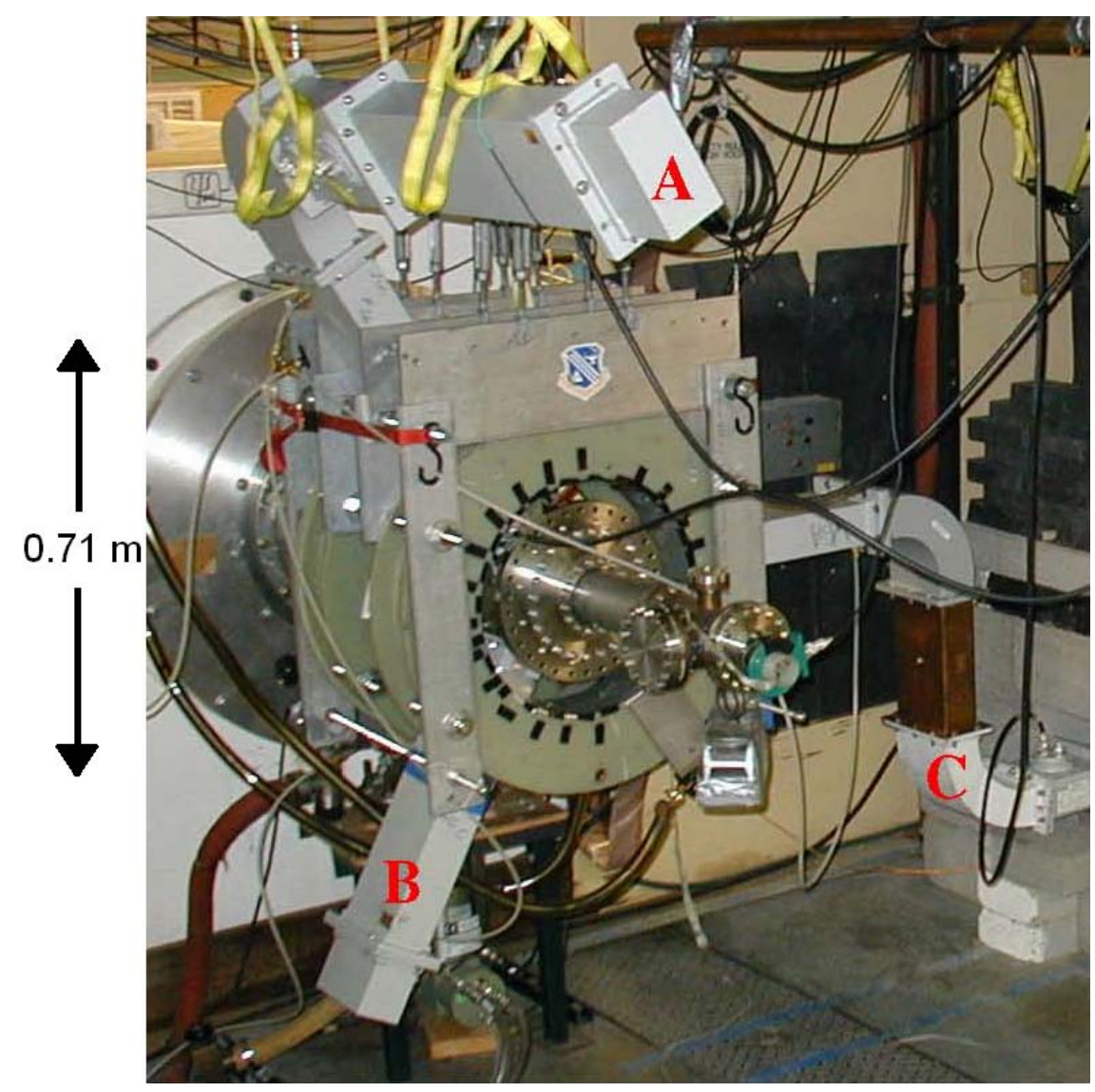

Figure 35. Photograph of the UM/L-3 Titan relativistic magnetron, showing attached waveguides in the unbalanced loading configuration used in the first magnetic priming experiment.

Waveguide-A utilized a -50dB microwave power coupler for diagnostics and a water load. Waveguide-B was terminated in a water load. Waveguide-C connected the relativistic magnetron to a $\mathrm{MW}$ radio frequency priming magnetron (not used in this experiment) through a T/R switch (for these experiments this waveguide was shorted through the center with a large-gauge metal rod to prevent power flow to the T/R switch and priming magnetron). It is important to note that this array of output waveguides and associated loads and shorts created asymmetric loading of the relativistic magnetron, not expected to be conducive to m-mode operation [55]. 
Figure 36 presents a schematic diagram detailing the diagnostics used during the first magnetic priming experiment.

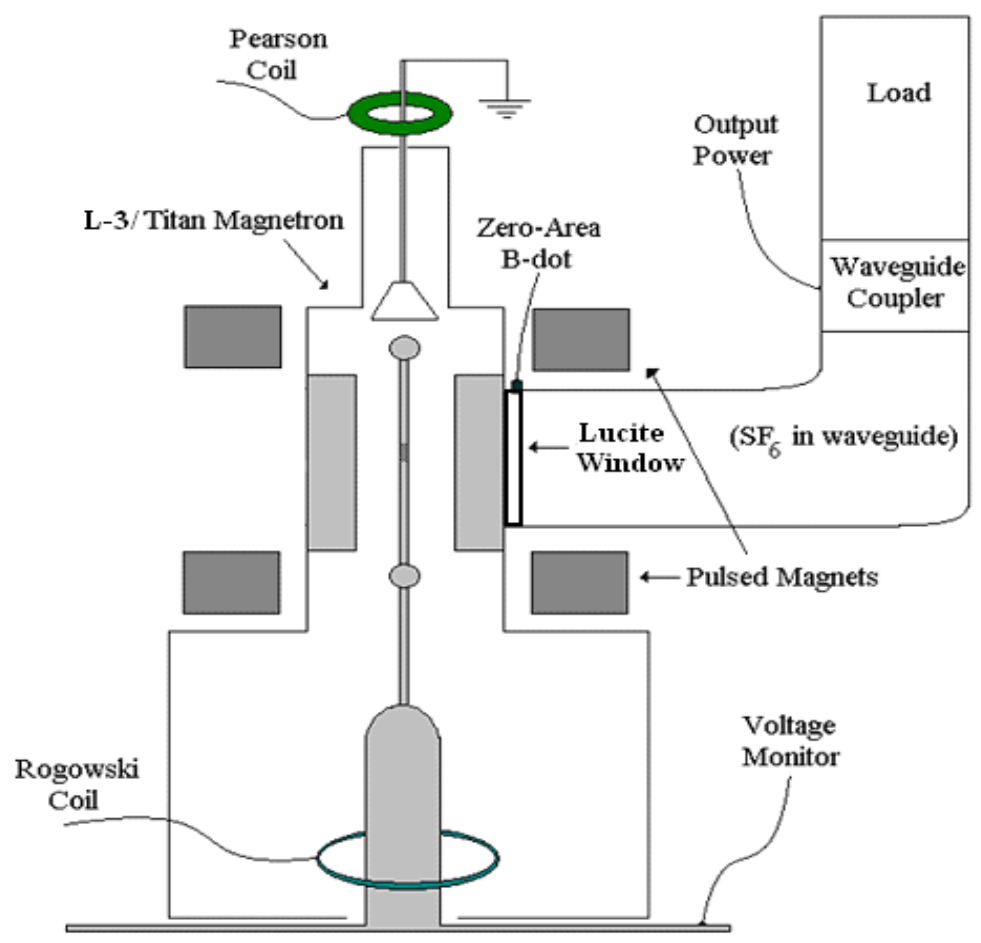

Figure 36. Schematic diagram of the UM/L-3 Titan relativistic magnetron experiment used in the first magnetic priming experiment, illustrating approximate locations of the experiment diagnostics (reproduced from Ref. [31]).

Only waveguide-A from Figure 36 is shown in this schematic. In this configuration, input current and voltage from MELBA are monitored by a Rogowski coil and copper-sulfate filled voltage-divider, respectively. Both diagnostics are incorporated into the Marx-Abramyan Bank tank and flange. A Pearson current transformer around the output lead for the end loss current collector detects the end loss current leaking out of the interaction space. Microwave power is sampled by a $-50 \mathrm{~dB}$ coupler in waveguide-A. The signal is then passed through a series of additional attenuators and measured by a 
microwave diode detector in the Faraday cage. The signal from the "zero-area" B-dot loop is heterodyned with a local oscillator, then run through a Time Frequency Analysis (TFA) algorithm [56].

Figure 37 shows the magnetron and attached waveguides in the balanced loading configuration employed in the second and third experiments.

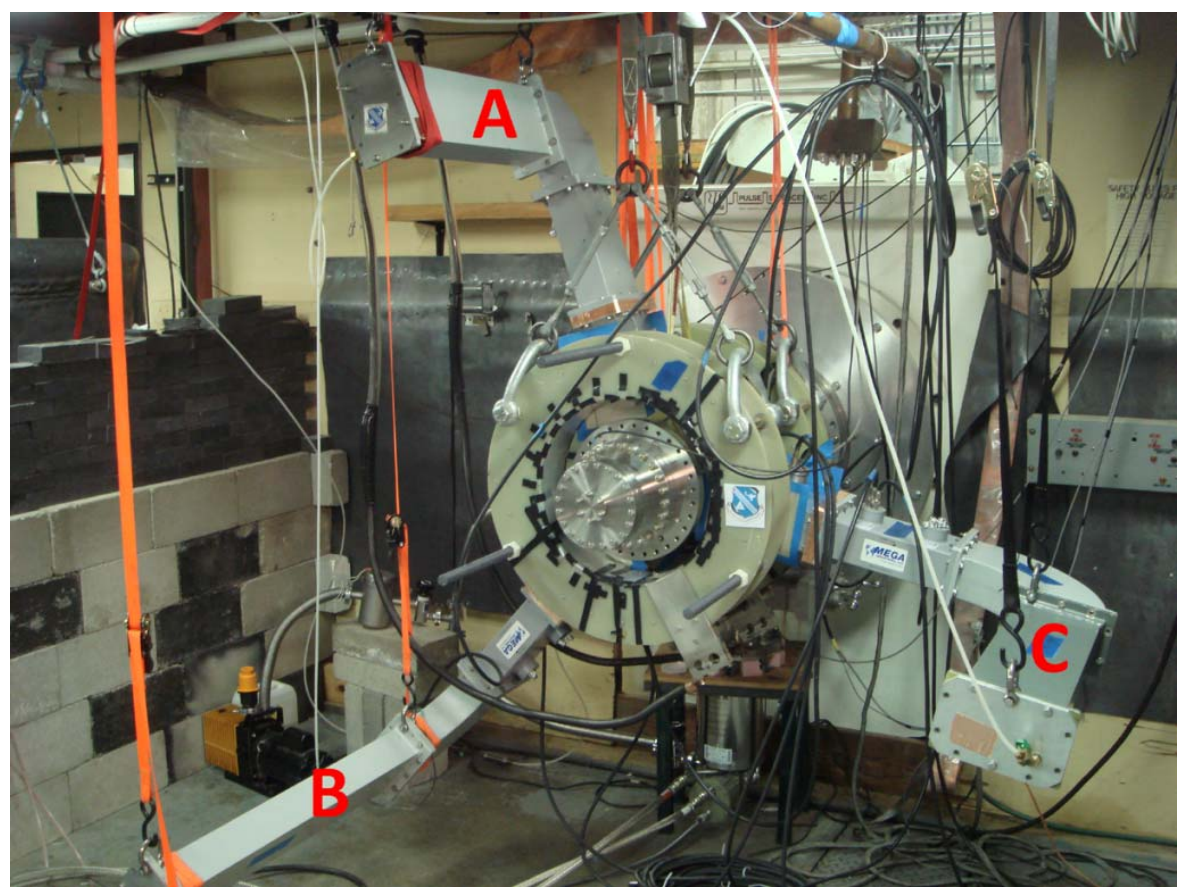

Figure 37. Photograph of the UM/L-3 Titan relativistic magnetron, showing attached waveguides in the balanced loading configuration used in the second and third magnetic priming experiment.

In the balanced loading configuration, each of the three waveguides included an Air Force Research Laboratory-built vacuum-side directional power coupler, followed by a metal-framed Lucite window, a $-50 \mathrm{~dB}$ air-side directional coupler, and a microwave load. Additional data on the AFRL vacuum-side power couplers, designed by M.D. Haworth, are available in Appendix A and Appendix 
B. In this configuration, the water loads (described in Ref. [31]) were replaced with better matched carbon fiber microwave loads (Eccosorb). Additional information on the Eccosorb microwave loads is provided in Appendix A.

A schematic diagram detailing the diagnostics utilized during the second and third magnetic priming experiments is displayed in Figure 38. Only waveguide A from Figure 37 is shown.

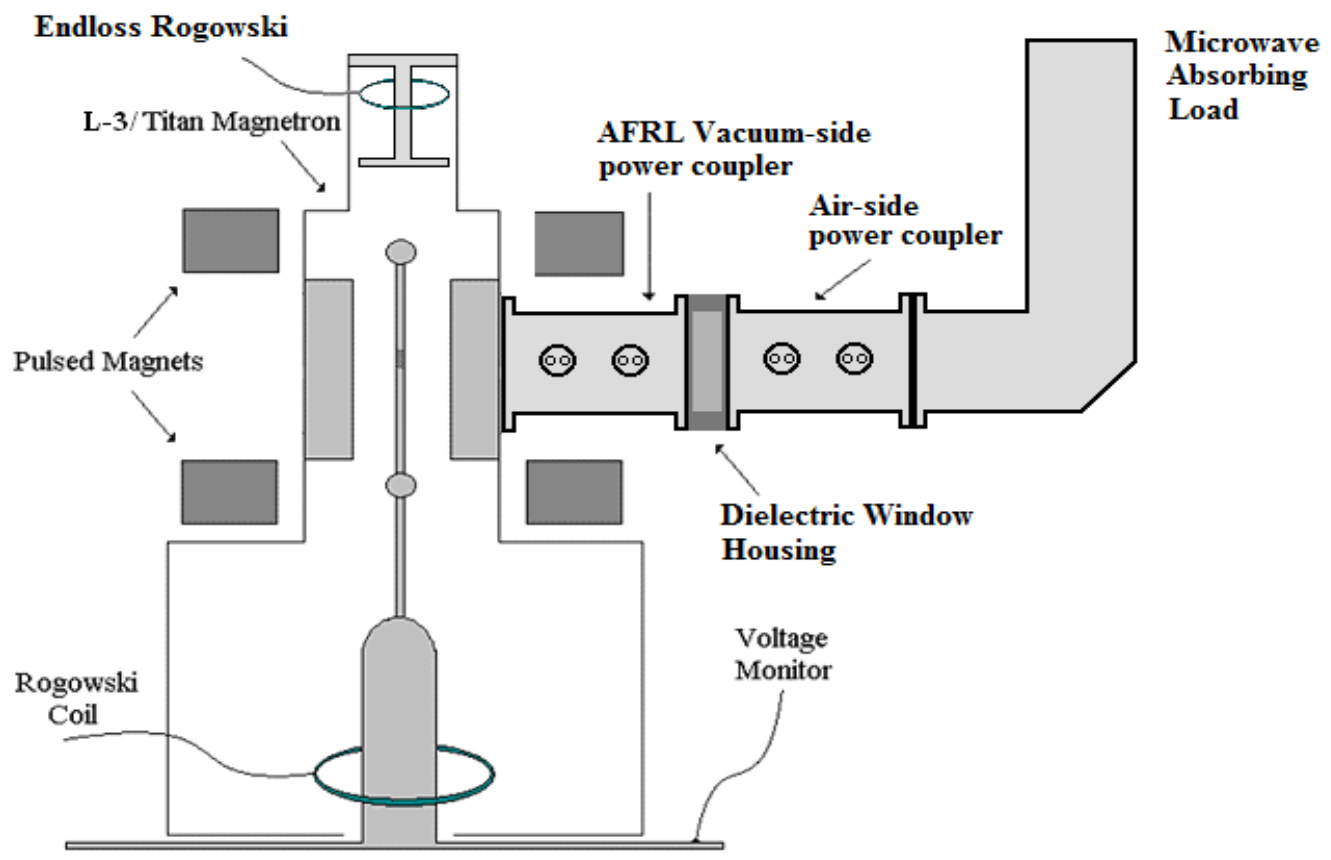

Figure 38. Schematic diagram of the UM/L-3 Titan relativistic magnetron experiment used in the second and third magnetic priming experiment, illustrating approximate locations of the experiment diagnostics.

As in the first experiment, input voltage was monitored using the copper sulfatefilled voltage divider contained in the MELBA oil tank. Magnetron entrance current was monitored with a smaller Rogowski coil located in the vacuum section between MELBA-C and the magnetron. The Pearson current transformer 
used for detecting end loss current in the original configuration was replaced with a Rogowski coil in the upgraded configuration. The newer input current and end loss current monitor Rogowski coils were found to have better time response to the current signals than did their counterparts in the first experiment. The microwave power was sampled from the vacuum side couplers in all three waveguides. These signals were then passed through a series of attenuators and band pass filters and measured by a microwave diode detector. The microwave frequency signal was also sampled from the vacuum side coupler on waveguide $\mathrm{C}$. This frequency signal was passed through a series of attenuators, heterodyned with a local oscillator, then analyzed with the TFA program previously mentioned. Photographs, connection diagrams, and calibration data for described diagnostics are available in Appendix A.

The modular cathode employed in this series of magnetic-priming experiments is detailed in Figure 39 and consisted of a grooved, aluminum support rod surrounded by an aluminum sleeve and connected between two electrostatic endcap-balls. The grooves were machined such that the magnetic wires placed in them were held against the inner wall of the cathode sleeve. Up to three magnetic wires of lengths ranging up to $27 \mathrm{~cm}$ could be mounted in the grooved support rod. 


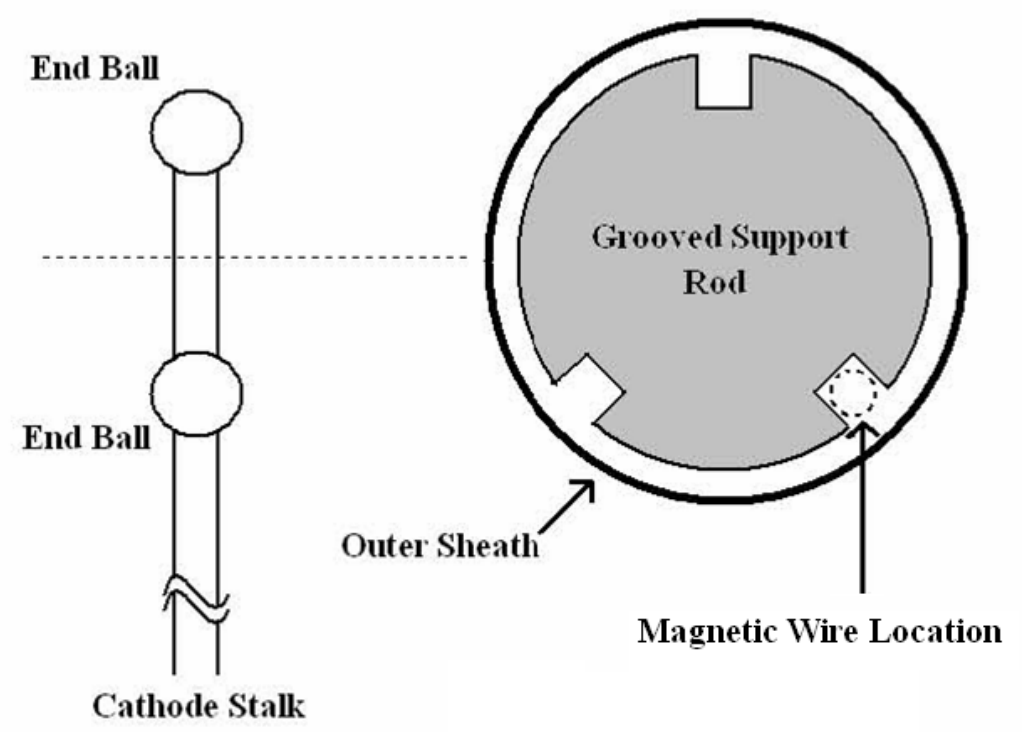

Figure 39. Illustration of the magnetic priming cathode, showing support rod geometry and magnetic wire locations.

The presence of the cathode sleeve allowed for the baseline and magnetic priming cases tested in this experiment to be electrostatically identical, so only the effects of the magnetic perturbations could be studied. The cathode sleeve itself had an outer diameter of $1.27 \mathrm{~cm}$ and a thickness of $0.086 \mathrm{~cm}$. The emission region on the cathode sleeve was etched by the Ablation-Line-Focus (ALF) process $[22,23,25]$. The cathode sleeve used in the first experiment was etched over a region that was $1.67 \mathrm{~cm}$ long and centered axially within the relativistic magnetron anode block. In the second and third experiments, the cathode sleeve was etched over a region that was $10 \mathrm{~cm}$ long and centered within the relativistic magnetron anode block. The magnetic priming portion of the cathode assembly was then attached to a cathode extension stalk connected to the output of MELBA-C. For the magnetically primed experiments, once 
loaded into the magnetron, the cathode was rotated such that the priming wires were angularly aligned with three of the anode vanes.

In order for the third magnetic priming experiment to be performed, the anode of the relativistic magnetron, shown in Figure 40 (a) and (b), was modified to allow magnetic wires to be placed within any of the vanes, directly below the surface of the anode.

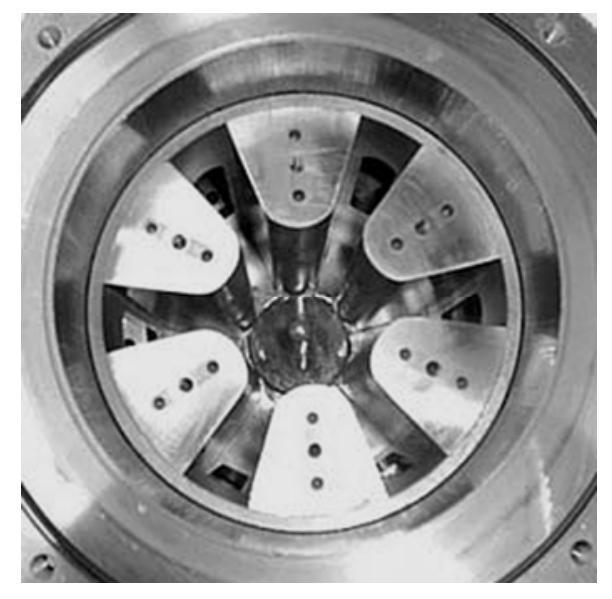

(a)

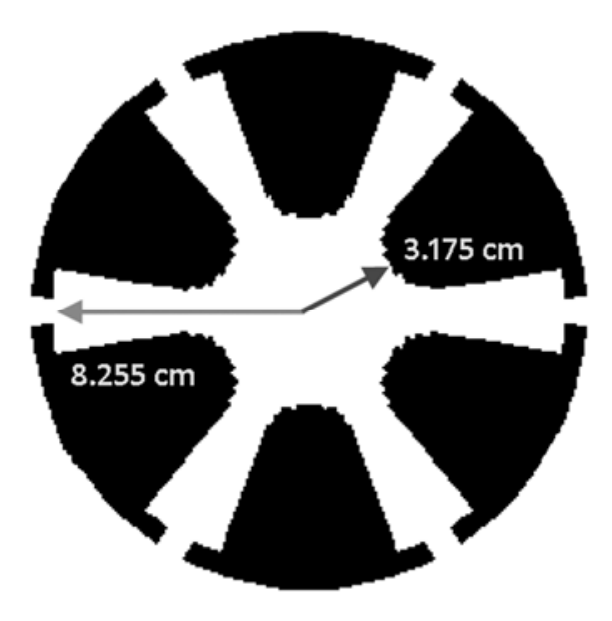

(b)

Figure 40. Unmodified UM/L-3 relativistic magnetron anode block (a) photo and (b) dimensions. 
A full-length $1.27 \mathrm{~cm}$ diameter hole was bored through each of the six $20.64 \mathrm{~cm}$ stainless steel anode vanes. A photograph of the modified anode block is displayed in Figure 41.

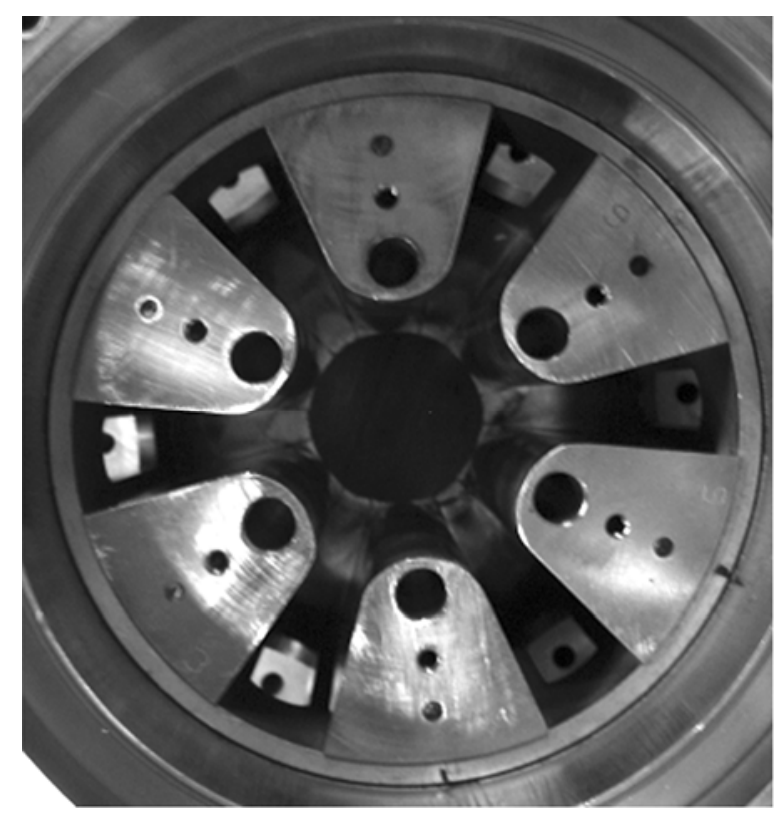

Figure 41. Modified UM/L-3 relativistic magnetron anode block showing $1.27 \mathrm{~cm}$ diameter boreholes in each of the anode vanes.

Each of the $0.508 \mathrm{~cm}$ diameter, high permeability nickel-iron alloy wires used in the magnetic priming at the anode experiment was mounted in a $1.27 \mathrm{~cm}$ diameter boron nitride rod which was then positioned within each of the anode boreholes. Figure 42 depicts a photograph of one of the boron nitride rods with a magnetic priming wire mounted. 


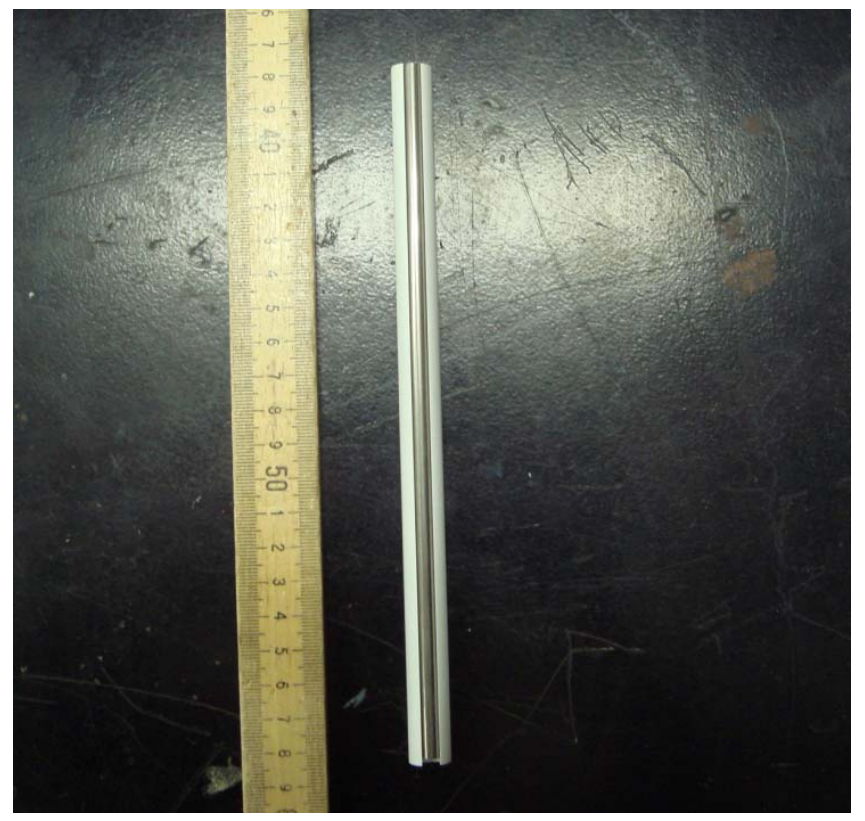

Figure $42.20 .64 \mathrm{~cm}$ long, $1.27 \mathrm{~cm}$ diameter boron nitride rod (white) with $0.508 \mathrm{~cm}$ diameter magnetic priming wire mounted.

Pulsed magnetic field measurements at the cathode surface found the perturbation to be about $2 \%$ of the baseline field for the $0.254 \mathrm{~cm}$ diameter cathode wires, and $1 \%$ for the $0.127 \mathrm{~cm}$ wires. The magnetic field perturbation at the surface of the anode vanes containing the magnetic wires was found to be about $2 \%$. Because both the cathode sleeve and anode vane wall separating the wires from the magnetron interaction space are thin and the magnet pulse is long ( $15 \mathrm{~ms})$, the shielding effect of the metal was minimal due to magnetic diffusion. This magnetic perturbation amplitude is weaker than that calculated by Neculaes et al. [12] for their oven magnetron priming experiments. Note, however, in the experiments involving magnetic priming at the cathode, this magnetic field perturbation is maximum on the cathode and decreases rapidly radially outward. This differs from Neculaes' experiments on oven magnetrons, as the magnetic perturbation in Ref. [12] is minimum on the cathode, and increases rapidly toward 
the anode. In both magnetic priming cases studied on the relativistic magnetron, the degree of magnetic priming in the present experiments is substantially weaker than that in either Neculaes' experiments or in the simulations performed by Jones [18] and Luginsland [21]. Even with the substantially weaker magnetic priming field in the present experiments on the relativistic magnetron, improvement over the unprimed case was observed, as shown in the next section.

\subsection{Experimental Results}

\subsubsection{Magnetic Priming at the Cathode (Unbalanced Loading)}

The two data sets taken during the first magnetic priming experiment were reported by Hoff et al. [57]. The first data set utilized the three, $4 \mathrm{~cm}$ long, 0.254 $\mathrm{cm}$ diameter nickel-iron wires for magnetic priming at the cathode. The second data set represents a baseline run with no magnetic wires in the cathode. Figure 43 depicts the parameter space of the magnetically primed and unprimed shots in relation to the Buneman-Hartree resonance lines for the $\pi$ and $2 \pi / 3$ modes. The RF mode phase velocities $\left(v_{p h}\right)$ used for the Buneman-Hartree resonance lines were calculated as $v_{p h}=\omega_{n} r_{a} / n$, where $\omega_{n}$ is the frequency of a given mode, as determined by a cold test of the magnetron, $r_{a}$ is the anode radius, and $n$ is the mode number. Cold test data for the $U M / L-3$ relativistic magnetron are provided in Appendix D. The MELBA-C Marx generator mean output voltage during the microwave pulses was measured to be $-315 \mathrm{kV}$ with a standard 
deviation of $9.8 \mathrm{kV}$. Magnetron shots were taken at magnetic fields ranging from 2.75 to 3.09 kGauss.

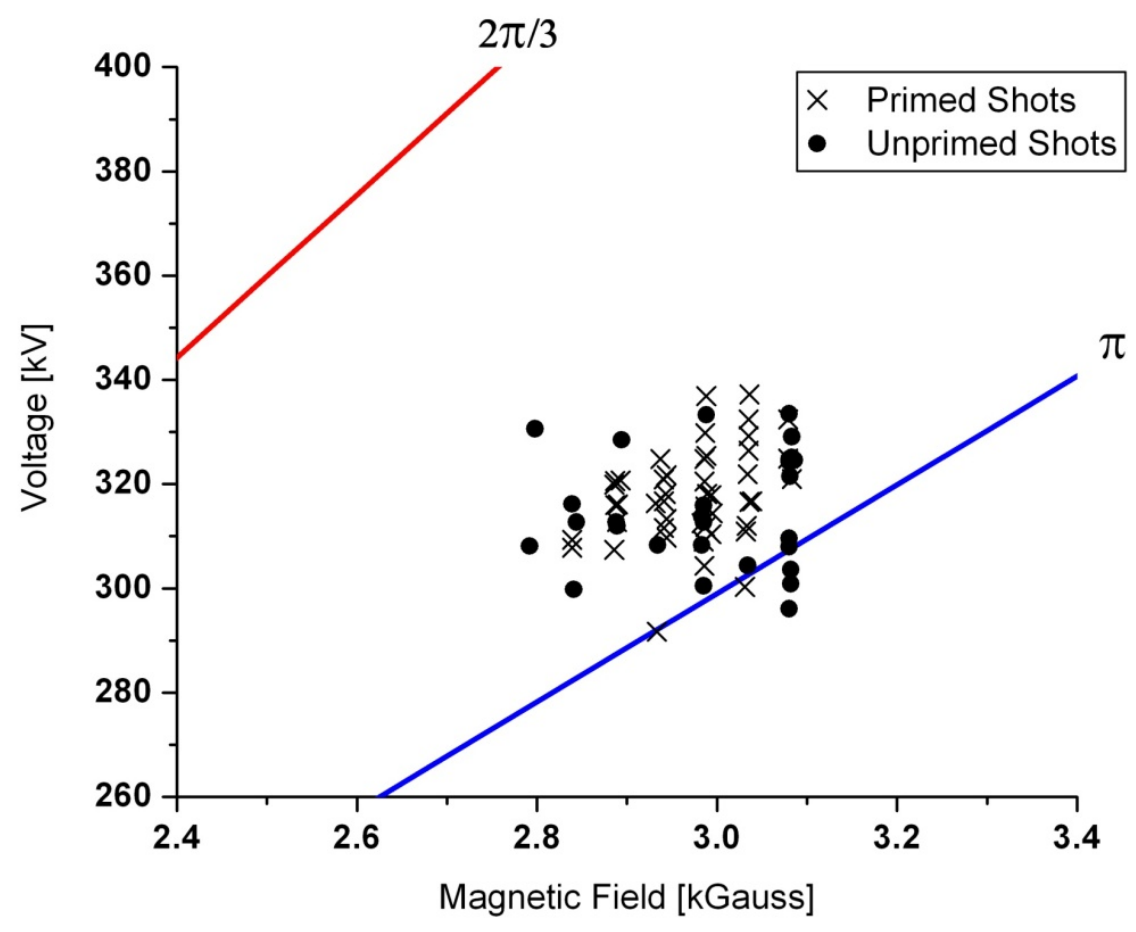

Figure 43. Experimental data points near the m-mode Buneman-Hartree resonance curve for the UM/L-3 Titan relativistic magnetron.

Figure 44 (a) and (b) present the voltage, current, microwave power, heterodyned microwave signal, and time-frequency analysis (TFA) for a typical magnetically primed shot (MELBA shot 11065). As noted previously, microwave power was measured on only one of the three output waveguides attached to the relativistic magnetron. Peak frequency for this shot was found to be $1009 \mathrm{MHz}$, in the range expected for $\pi$-mode oscillation. 
Voltage, Entrance Current, and Power for Shot 11065

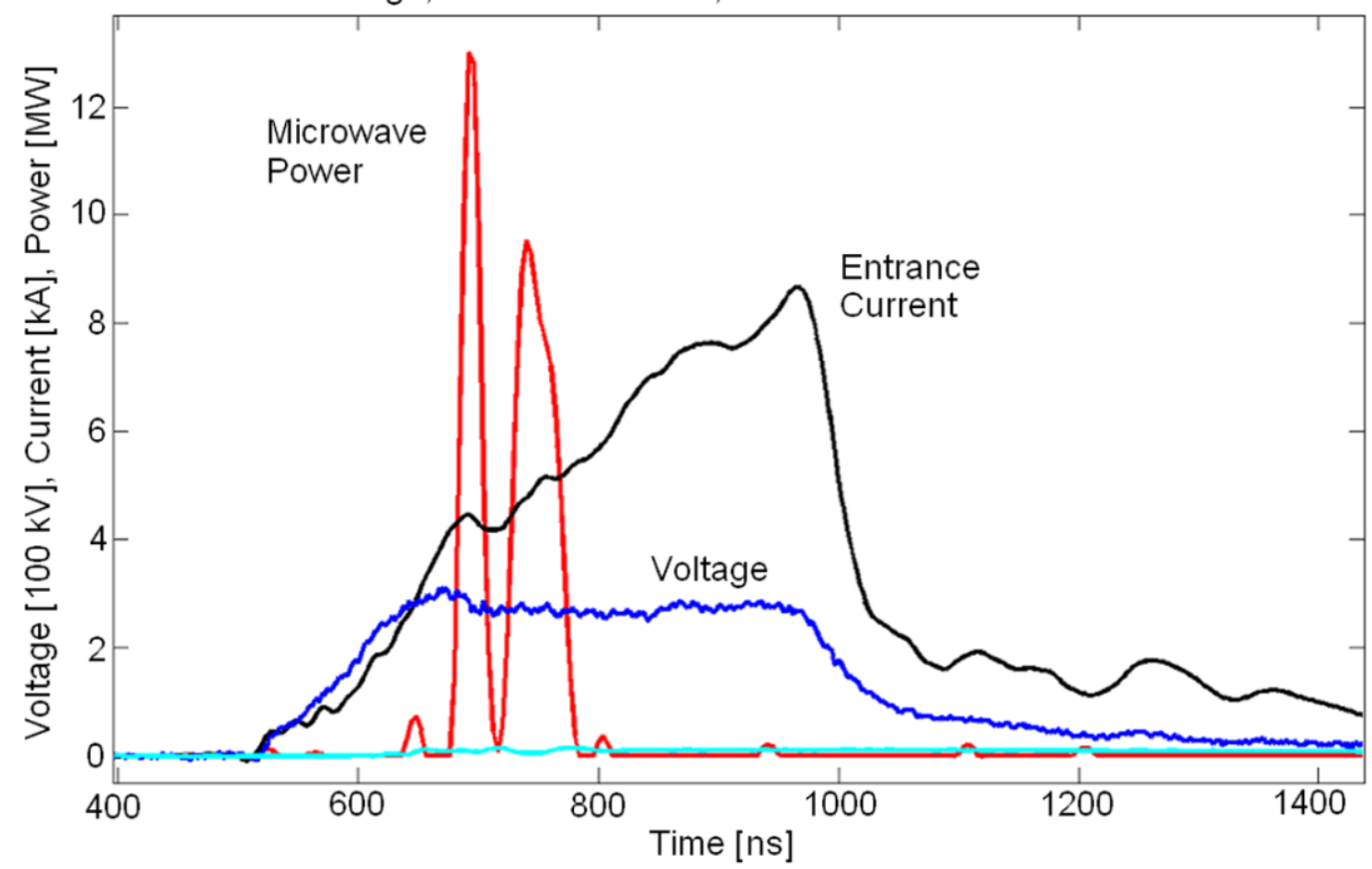

(a)
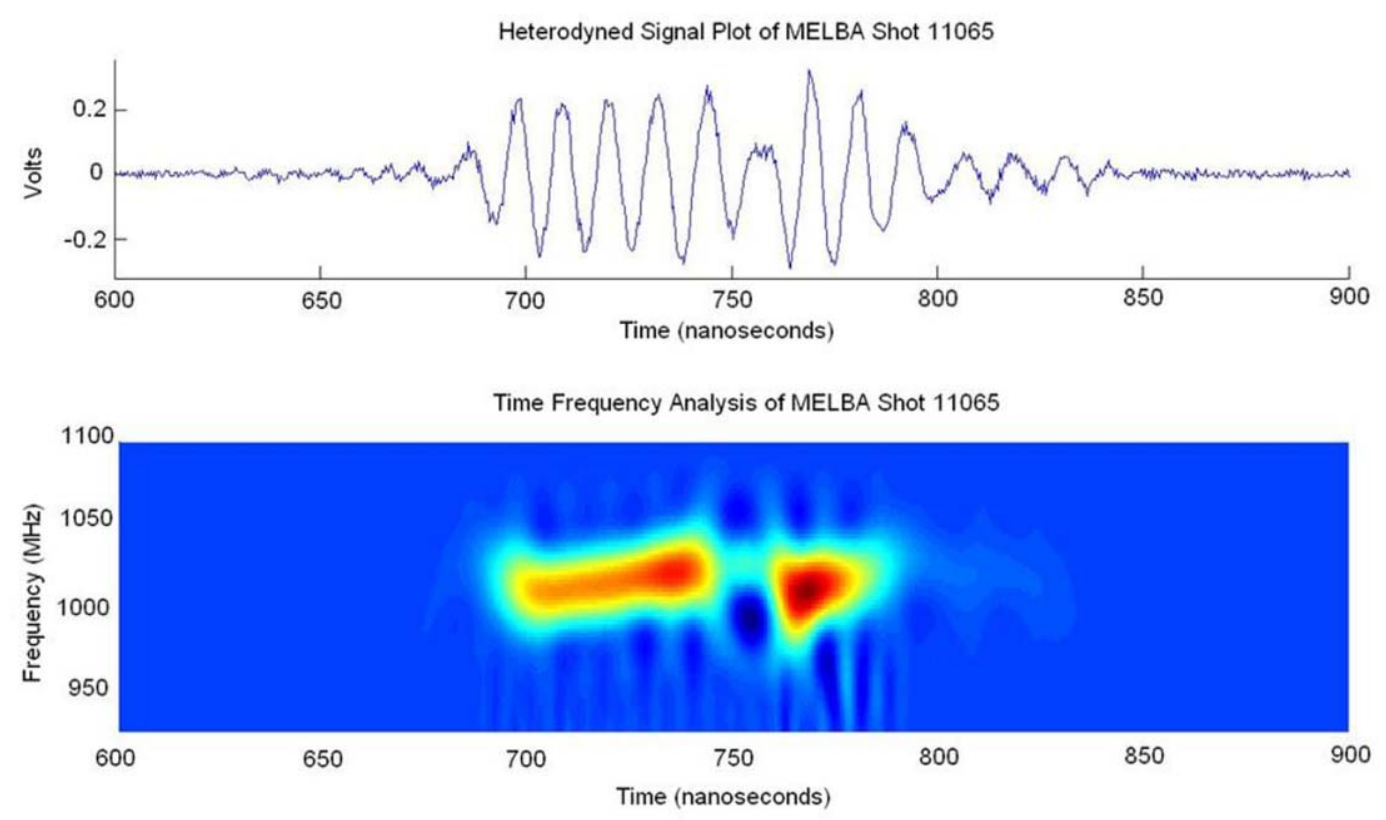

(b)

Figure 44. (a) Microwave power, incoming diode current, and diode voltage for a magnetically primed magnetron shot (shot 11065). The unlabled light blue trace in (a) is from a diagnostic not used in these experiments. (b) (upper) Magnetron heterodyne microwave signal (local oscillator set to $1.1 \mathrm{GHz}$ ) and (lower) corresponding TFA plot 
The frequency of the microwave pulse at peak power, plotted as a function of applied magnetic field, is shown for the primed-versus-unprimed data sets in Figure 45 (a) and (b), respectively. When evaluated over each entire data set, the magnetically primed relativistic magnetron operated in the $\pi$-mode for 57 percent of the shots. In the baseline case, the relativistic magnetron operated in the $\pi$-mode in only 42 percent of the shots. The $15 \%$ increase in $\pi$-mode shots in the magnetically primed configuration versus the baseline configuration demonstrates that magnetic priming at the cathode increases the probability of the magnetron starting up in a desired mode (in this case, the m-mode) instead of a less desirable competing mode (specifically, the $2 \pi / 3$-mode). 


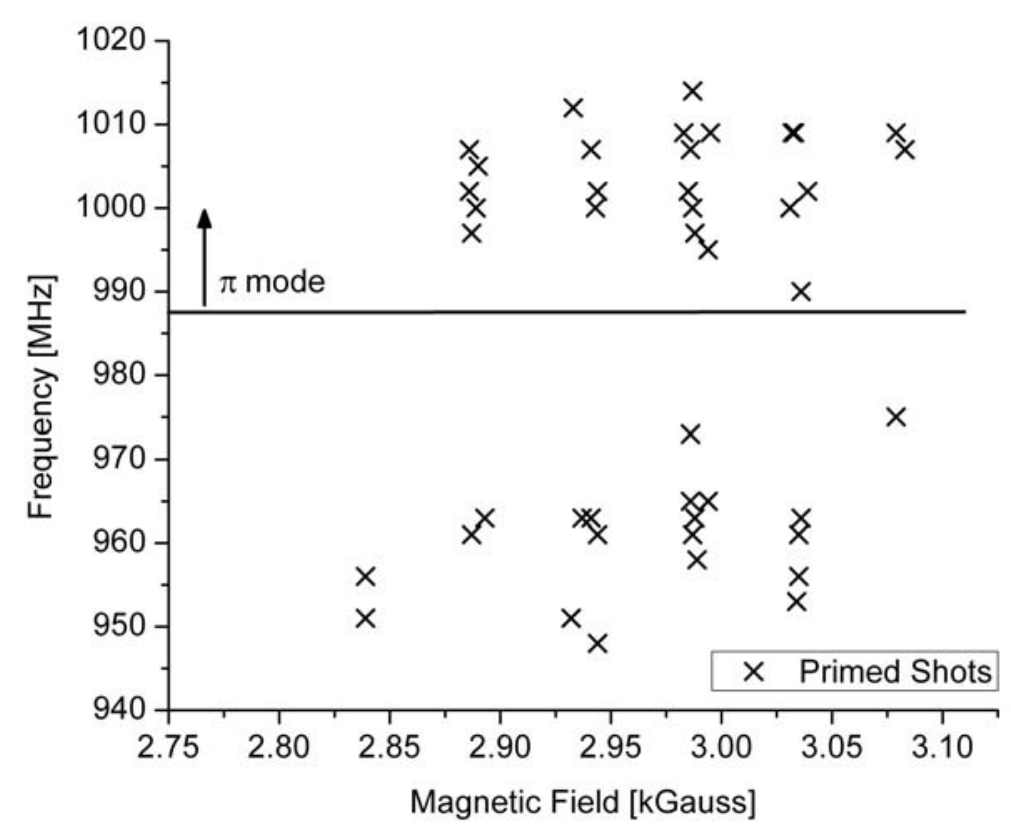

(a)

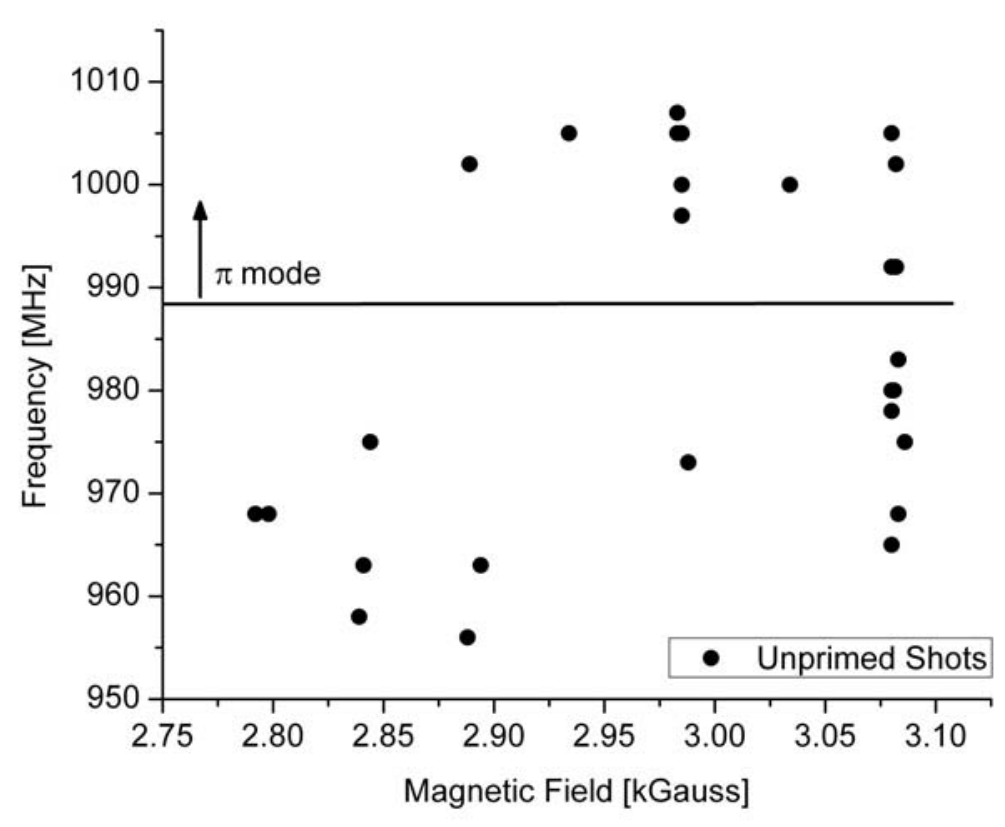

(b)

Figure 45. (a) Microwave frequency at peak power as a function of applied magnetic field for the magnetically primed UM/L-3 Titan relativistic magnetron. (b) Microwave frequency at peak power as a function of applied magnetic field for the unprimed UM/L-3 Titan relativistic magnetron. All shots below the $\pi$-mode demarcation line are considered $2 \pi / 3-$ mode shots 
Figure 46 (a) displays a plot of peak microwave power versus pulse width for all $\pi$-mode shots in the magnetically primed and unprimed configurations. In general, the $\pi$-mode shots in the magnetically primed case exhibited higher peak microwave power and longer pulses than the unprimed baseline case. Figure 46 (b) depicts the mean values for the peak microwave power and pulse width. Error bars extend one standard deviation from the mean. Differences in peak microwave power and pulse width between $\pi$-mode data sets for the primed and unprimed cases were verified to be statistically significant to a significance level of 0.05 , using a t-test. From this plot it is evident that the magnetically primed $\pi-$ mode shots exhibited, on average, nearly double the peak power of the unprimed shots (11.4 MW primed versus 6.5MW unprimed) in the single output waveguide. Additionally, the mean microwave pulse width in the magnetically primed case was extended by approximately $15 \mathrm{~ns}$ ( $\sim 30$ percent) over the baseline case. 


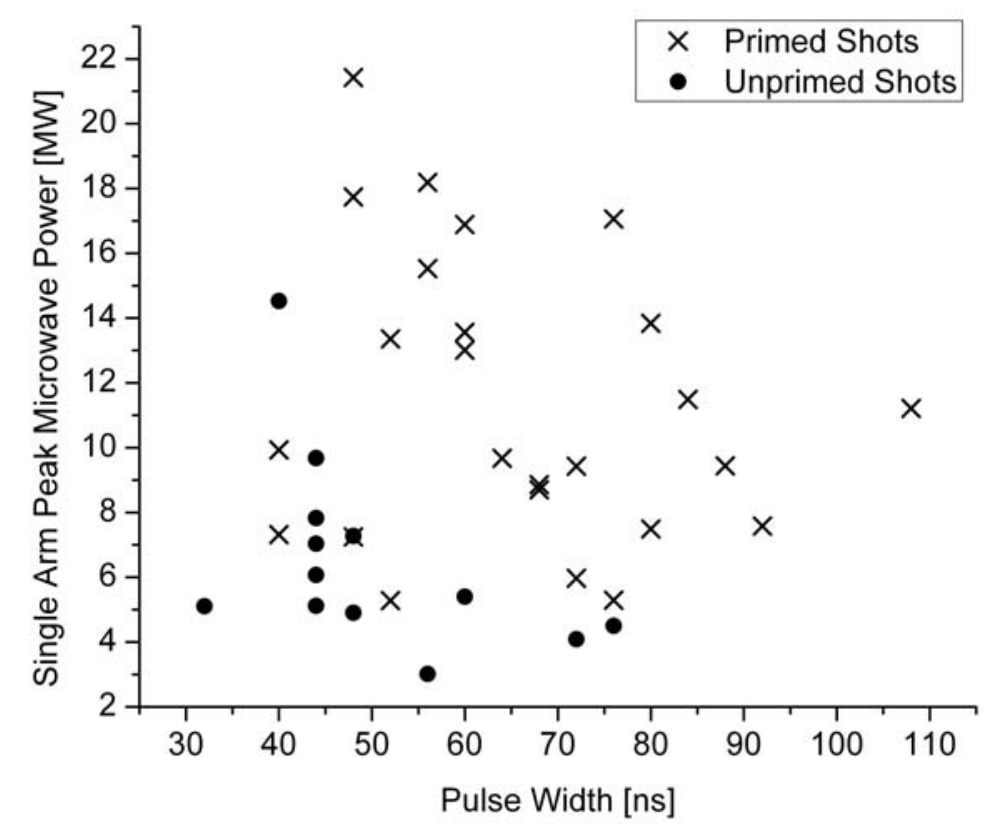

(a)

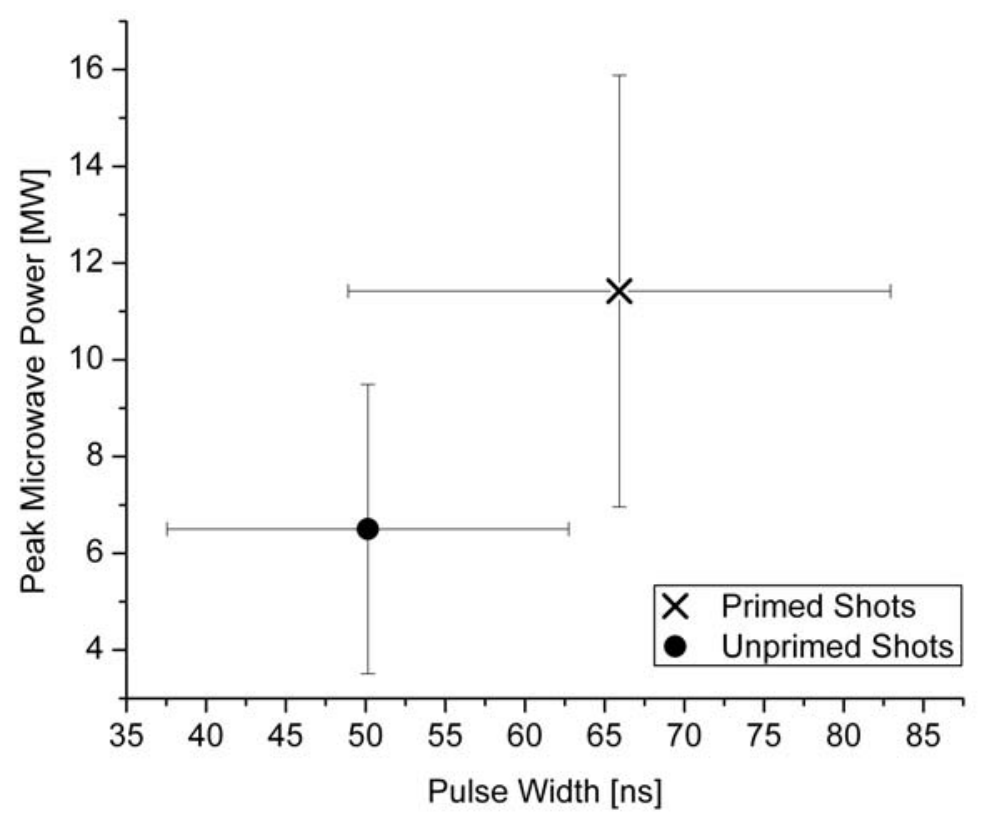

(b)

Figure 46. (a) Peak microwave power versus pulse width for all $\pi$-mode shots in the magnetically primed versus unprimed configurations. (b) Mean values of peak microwave power versus pulse width for the magnetically primed versus unprimed configurations. 
In addition to showing longer microwave pulse widths, the magnetically primed relativistic magnetron also showed reductions in starting time and time to peak power for m-mode shots. For these experiments, magnetron startoscillation (starting) time was defined as the time at which the MELBA-C voltage pulse reached $10 \%$ of maximum value until the time that the heterodyned microwave signal became detectable above the baseline noise. Time to peak power was defined as the time at which the MELBA-C voltage pulse reached $10 \%$ of maximum value until the time that the measured microwave signal reached maximum amplitude. Plots comparing the magnetron m-mode starting time and time to peak power of the magnetically primed and unprimed data sets are shown in Figure 47. Error bars extend out to one standard deviation of the mean value. Differences between the primed and unprimed sets in starting time and time to peak power were verified to be statistically significant to a significance level of 0.05 using a t-test. Mean starting time for the magnetically primed case was found to be less than that of the baseline case by $42 \mathrm{~ns}(27 \%)$. The magnetically primed case also showed a modest reduction of $33 \mathrm{~ns}(12 \%)$ in time to peak power when compared to the unprimed baseline case. 


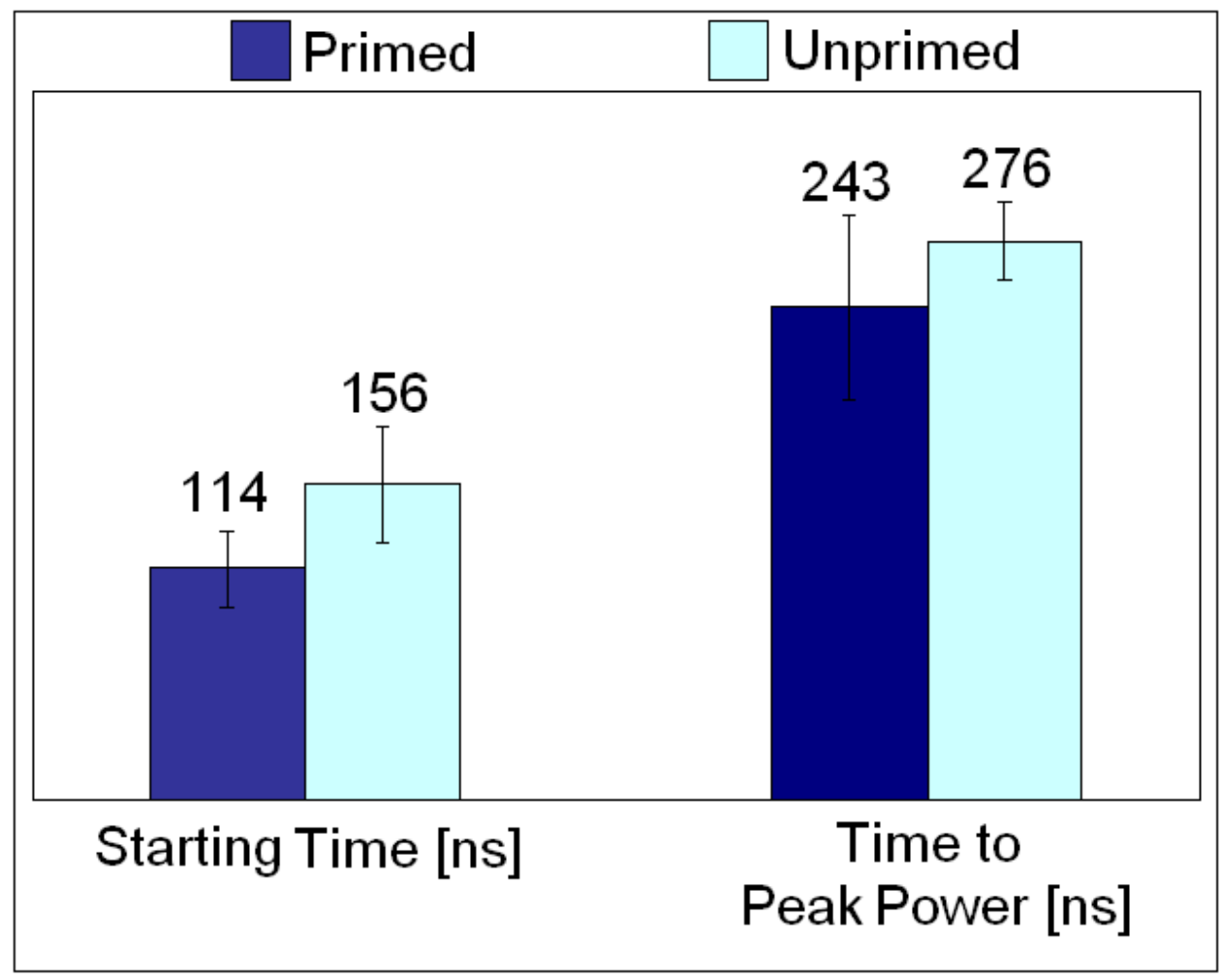

Figure 47. Mean values of magnetron $\pi$-mode oscillation starting time and time to peak power for the magnetically primed versus unprimed data sets.

Mean magnetron starting-currents for the magnetically primed and unprimed $\pi$-mode shots are displayed in Figure 48. For this experiment, starting current was defined as the value of the magnetron entrance current at the time that the heterodyned microwave signal became detectable above the baseline noise. Starting currents in the magnetically primed case were significantly lower than those in the unprimed case. Mean starting current for the primed data set was found to be $1.04 \mathrm{kA}$ with a standard deviation of $0.40 \mathrm{kA}$. Mean starting current for the unprimed data set was measured to be $1.50 \mathrm{kA}$ with a standard deviation of $0.37 \mathrm{kA}$. At a significance level of 0.05 , the difference in mean starting current between these two data sets was verified to be statistically 
significant using a t-test. Thus, for this experiment, magnetic priming was shown to reduce the mean magnetron starting current to $69 \%$ of the unprimed baseline value.

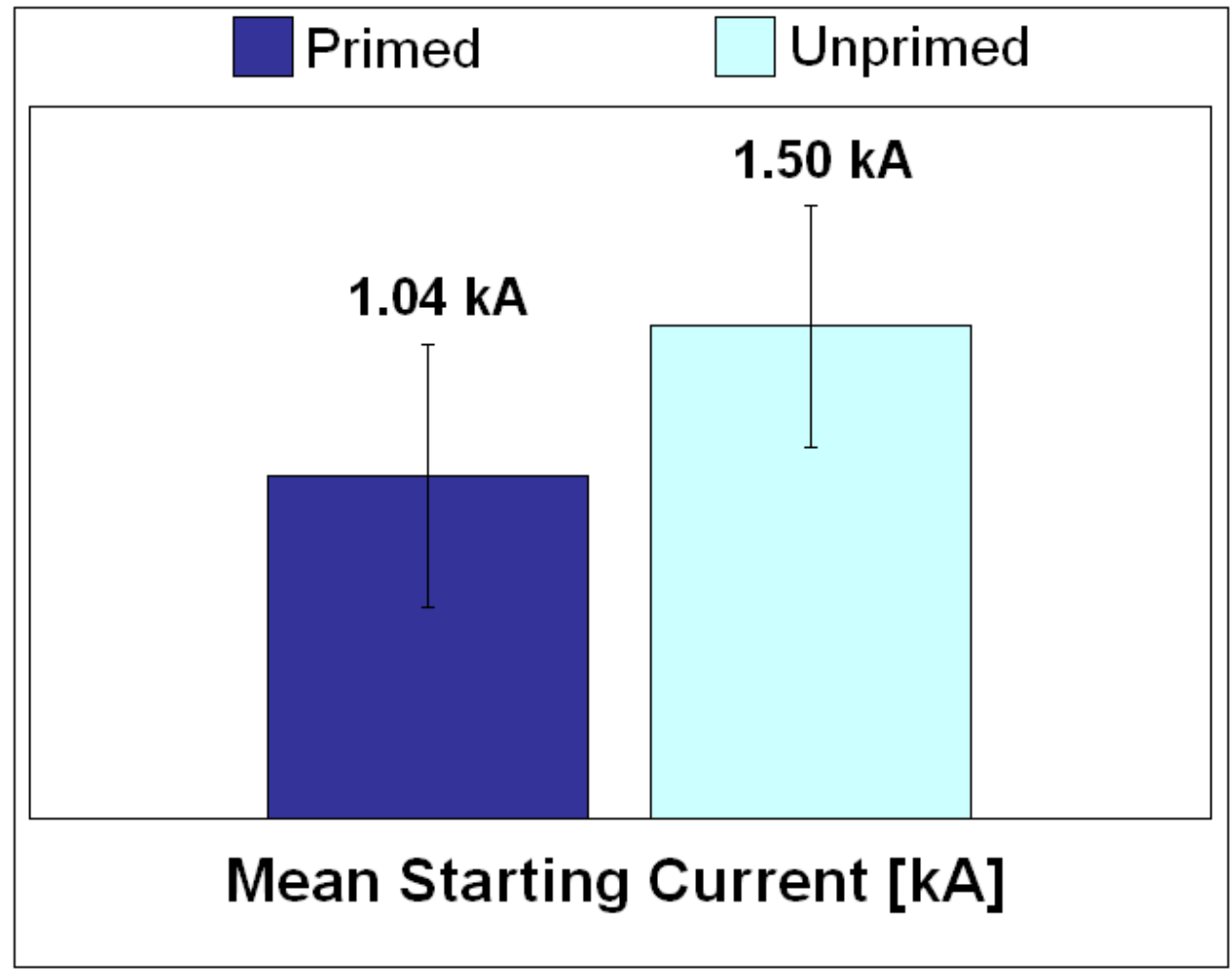

Figure 48. Magnetron starting current for $\pi$-mode shots in the magnetically primed versus unprimed cases.

\subsubsection{Magnetic Priming at the Cathode (Balanced Loading)}

The second magnetic priming data set consisted of six magnetically primed cases, with $4 \mathrm{~cm}, 8 \mathrm{~cm}, 12 \mathrm{~cm}, 16 \mathrm{~cm}, 20 \mathrm{~cm}$, and $27 \mathrm{~cm}$ length sets of three, $0.127 \mathrm{~cm}$ diameter wires spaced 120 degrees apart and embedded in the cathode. An additional unprimed data set was used as a control set for comparison. Figure 49 (a) through (g) depict the voltage and magnetic field parameter space of the magnetically primed cases as well as the unprimed 
baseline set in relation to the Buneman-Hartree resonance lines for the $\pi$ and

$2 \pi / 3$ modes. Additional plots for frequency versus magnetic field are provided in Appendix C.

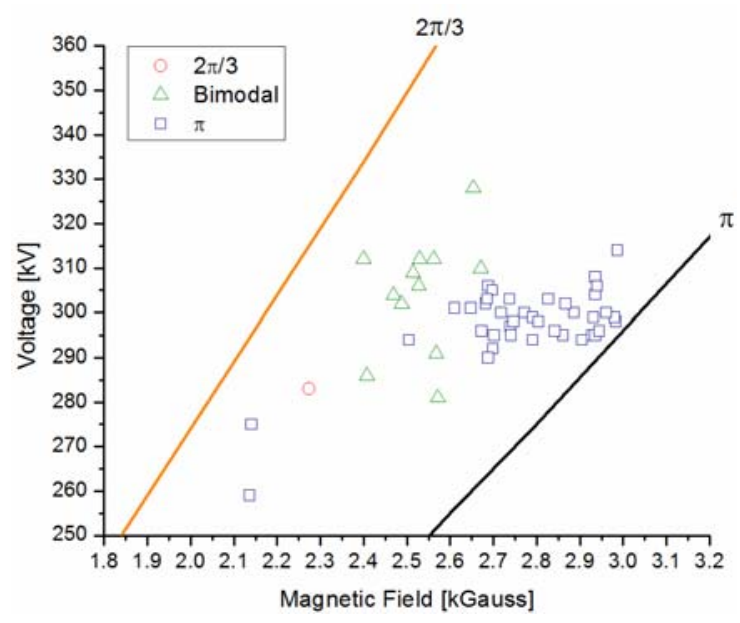

(a)

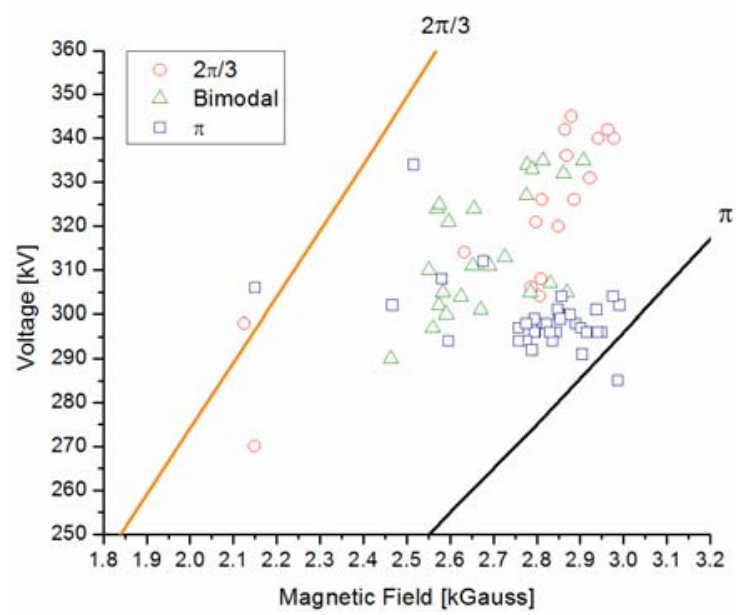

(c)

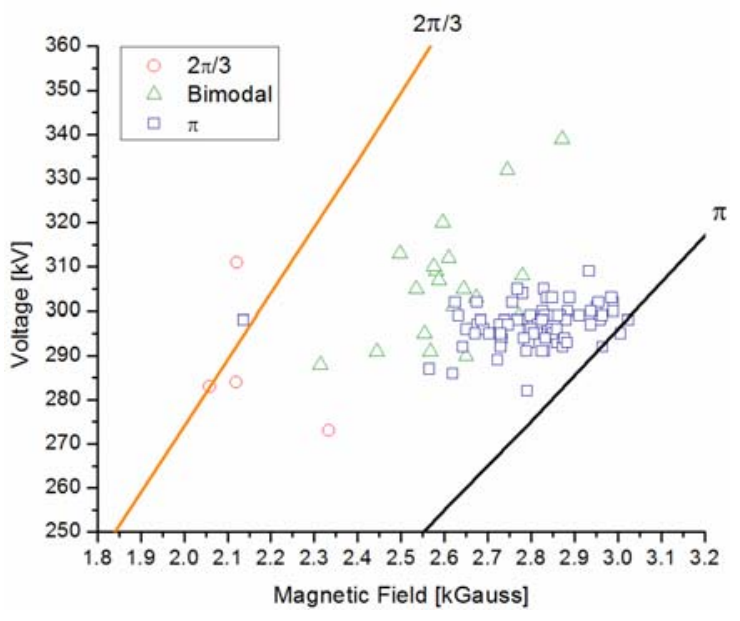

(b)

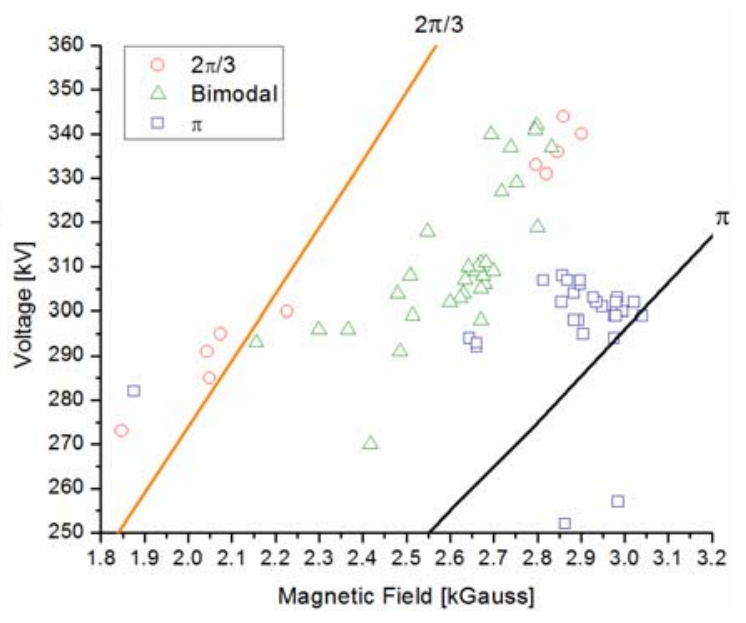

(d) 


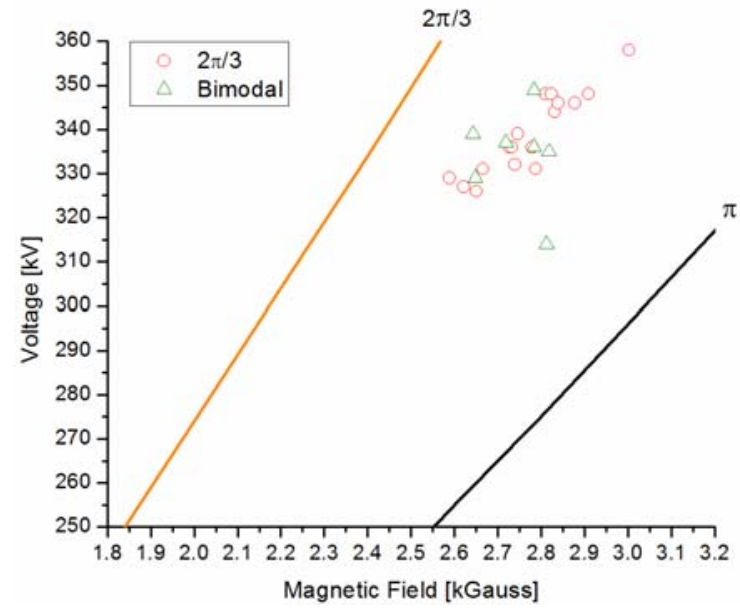

(e)

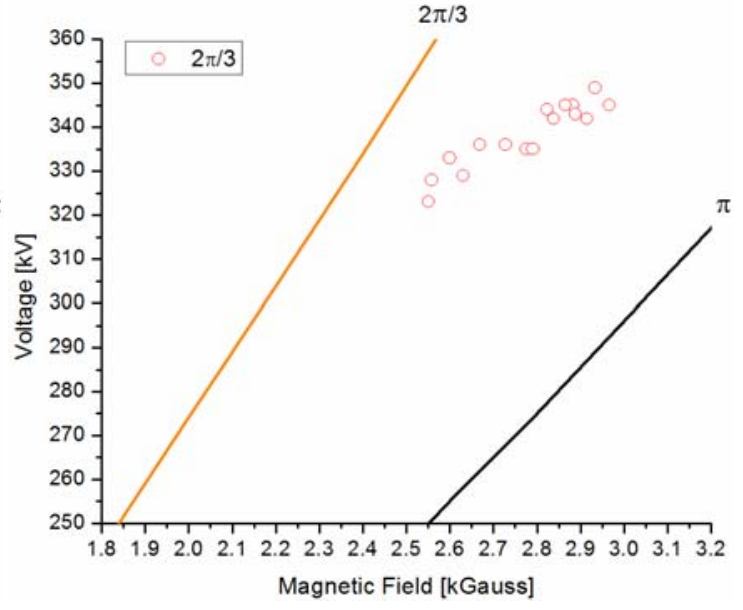

(f)

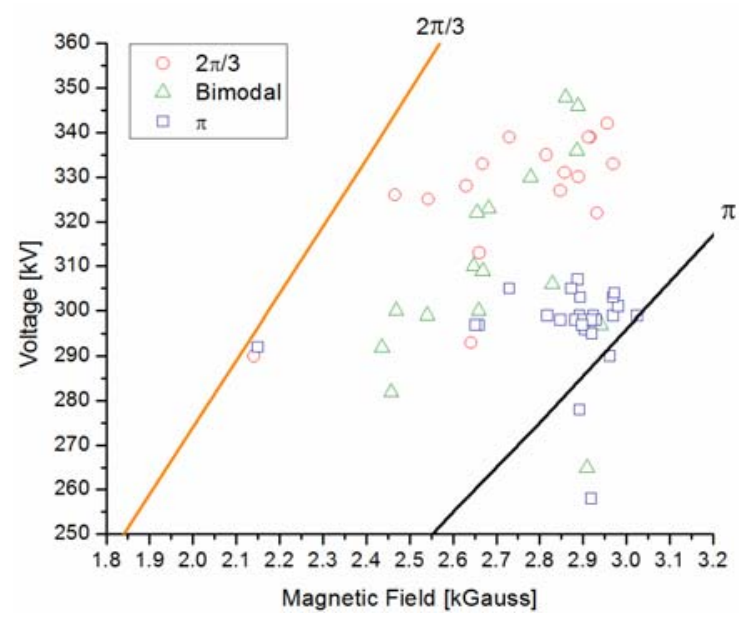

(g)

Figure 49. Experimental data points near the $2 \pi / 3$ and $\pi$-mode Buneman-Hartree resonance curves for the UM/L-3 Titan relativistic magnetron. (a) $4 \mathrm{~cm}$ cathode wires, (b) 8 $\mathrm{cm}$ cathode wires, (c) $12 \mathrm{~cm}$ cathode wires, (d) $16 \mathrm{~cm}$ cathode wires, (e) $20 \mathrm{~cm}$ cathode wires, (f) $27 \mathrm{~cm}$ cathode wires, (g) unprimed (no wires)

It is important to note that the voltage ordinate for each of the data points in Figure 49 (a) through (g) was determined by averaging the reading of the input voltage diagnostic over the duration of each of the microwave pulses. Although the voltage output from MELBA-C is nominally a $300 \mathrm{kV}$ "flat top" pulse, the actual pulses tend to overshoot by up to $15 \%$ and then trend downward to a few 
percent below $300 \mathrm{kV}$ until the termination of the voltage output by the crowbar. On average, the $2 \pi / 3$-mode microwave pulses occurred greater than 50 ns earlier in time than the m-mode microwave pulses. Although the voltage pulses for all magnetron shots in this experiment were approximately the same, when compared to the $\pi$-mode oscillation, the $2 \pi / 3$-mode tends to oscillate at higher average voltages during the duration of the microwave pulses. This behavior is consistent with the Buneman-Hartree conditions plotted above.

The formation of plasma around the cathode can affect the Hull and Buneman-Hartree conditions. Cathode plasma increases the effective cathode radius, thus decreasing the effective gap spacing. A decrease of the overall gap spacing will result in a reduction of the slope of the Hull and Buneman-Hartree curves. The type of etched cathode used in these experiments have been shown to form plasma under certain operating conditions [22]. The Buneman-Hartree and Hull conditions will also be modified by effects of diamagnetic current [58] and axial current [59].

The bimodal shots shown in Figure 49 are defined as magnetron shots where the magnetron started oscillating in one mode then jumped to a different mode after a brief period of no oscillation. In $97 \%$ of the bimodal shots, the magnetron started up in the $2 \pi / 3$-mode then jumped to the $\pi$-mode. Figure 50 (a) and (b) present the voltage, current, microwave power, heterodyned microwave signal, and time-frequency analysis (TFA) for one of the bimodal magnetron shots without magnetic priming. Microwave power was summed over all three of the output waveguides. 


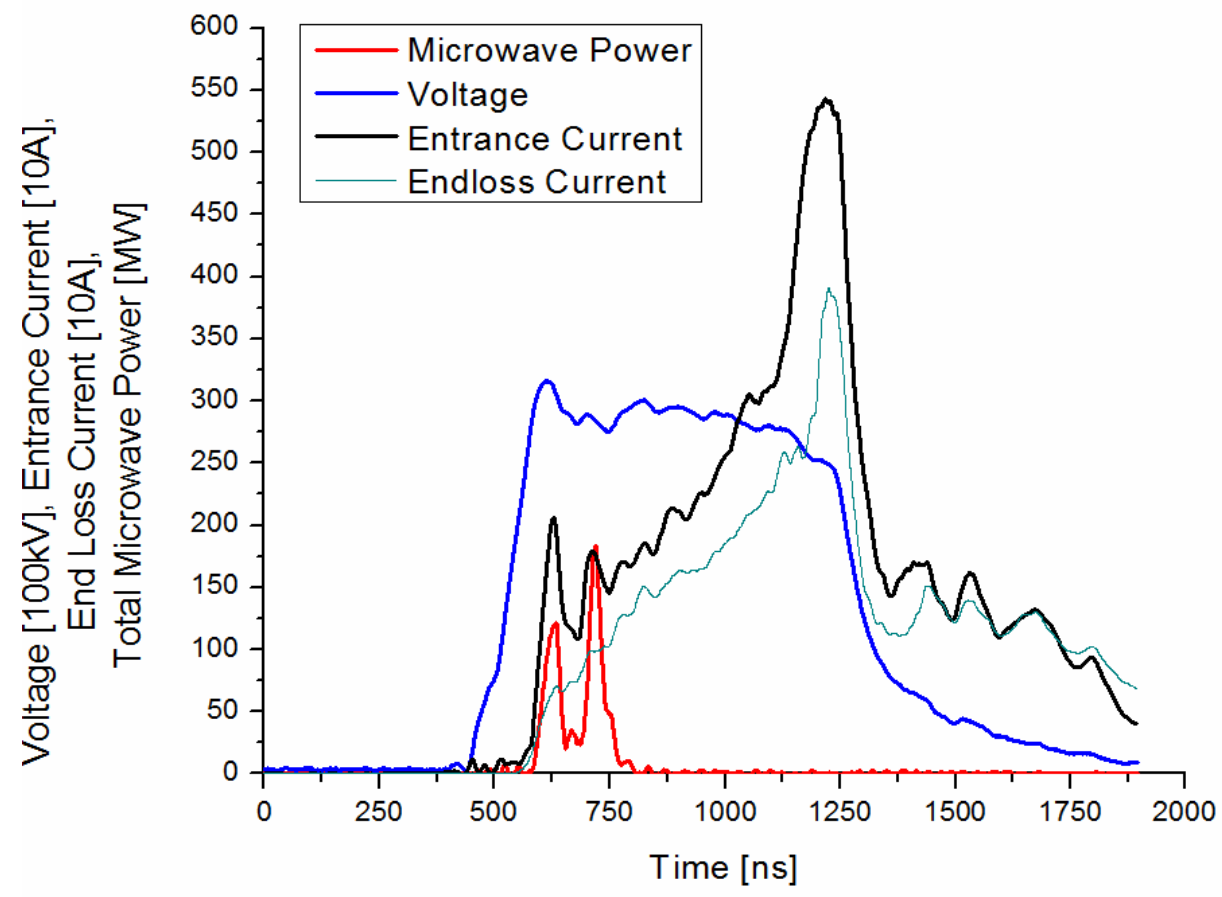

(a)

Heterodyned Signal Plot of MELBA Shot 12428
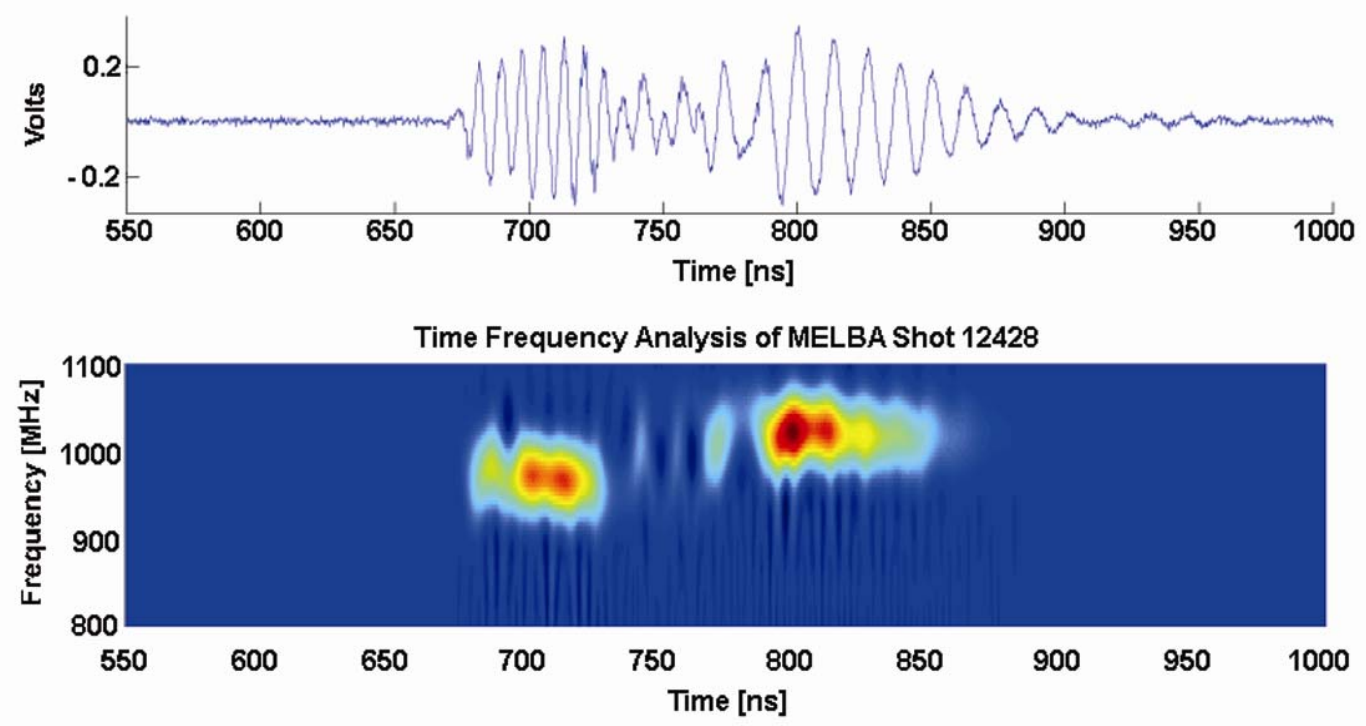

(b)

Figure 50. (a) Microwave power, incoming diode current, end loss current, and diode voltage for an unprimed bimodal magnetron shot (shot 12428). (b) (upper) Magnetron heterodyne microwave signal (local oscillator set to $1.1 \mathrm{GHz}$ ) and (lower) corresponding TFA plot 
The magnetic priming cases utilizing the $4 \mathrm{~cm}$ and $8 \mathrm{~cm}$ wires, depicted in Figure $49(a)$ and (b), had better $\pi$-mode performance $(74 \%$ and $76 \% \pi$-mode shots) than the baseline case ( $45 \% \pi$-mode shots). Additionally, the $2 \pi / 3$-mode was almost entirely suppressed in the 4 and $8 \mathrm{~cm}$ wire cases. This is in contrast to the $20 \mathrm{~cm}$ and $27 \mathrm{~cm}$ wire cases (Figure 49 (e) and (f)) in which no shots occur in the $\pi$-mode. Shot percentages are graphically depicted in Figure 51.

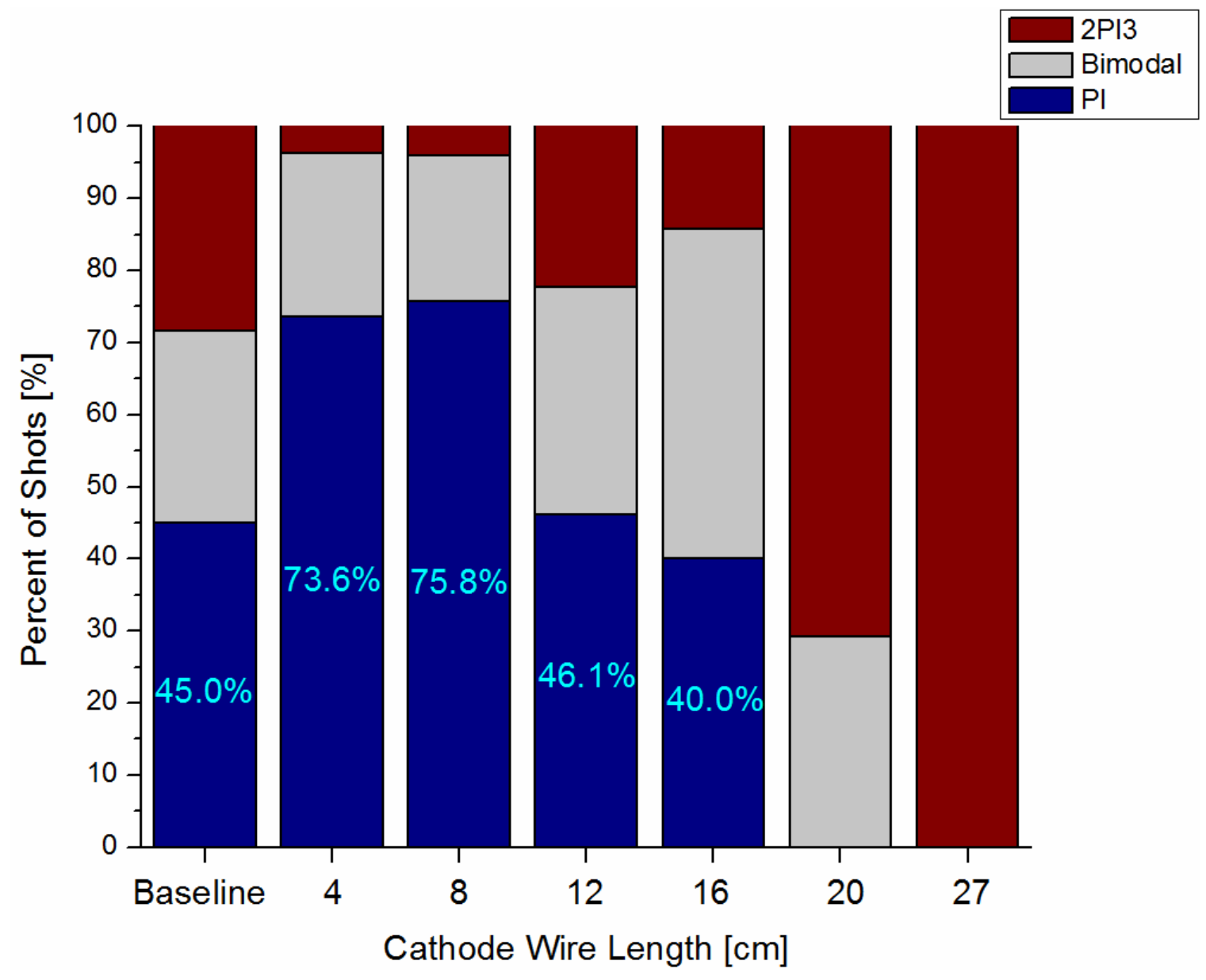

Figure 51. Shot percentages for the case of magnetic priming at the cathode with balanced loading.

Figure 52 (a) displays mean peak microwave power versus mean pulse width for all m-mode shots observed in the second magnetic priming experiment. 
Mean peak microwave power versus mean pulse width is plotted for all $2 \pi / 3-$ mode shots in Figure 52 (b). At a significance level of 0.05 , the differences in $\pi-$ mode mean peak microwave power and mean pulse width between the $8 \mathrm{~cm}$ wire, $12 \mathrm{~cm}$ wire, and $16 \mathrm{~cm}$ wire cases and the unprimed baseline case could not be shown to be statistically significant by a t-test. When compared with the baseline case, the $4 \mathrm{~cm}$ wire case, however, was shown to have statistically significant reductions in both peak total power (259 MW primed versus $384 \mathrm{MW}$ unprimed) and pulse width (152 ns primed versus 196 ns unprimed) for $\pi$-mode shots.

In the cases of the $4 \mathrm{~cm}, 8 \mathrm{~cm}$ and $12 \mathrm{~cm}$ wires, a statistical reduction in the peak power of the $2 \pi / 3$-mode microwave pulses was verified to a confidence level of 0.05 , using a t-test. The $4 \mathrm{~cm}, 8 \mathrm{~cm}$, and $12 \mathrm{~cm}$ wire cases had mean total power values of $46 \mathrm{MW}, 68 \mathrm{MW}$, and $87 \mathrm{MW}$, respectively, for $2 \pi / 3$-mode shots, while the unprimed case had a mean total power of 108 MW. Statistically significant reductions in pulse width from the unprimed case for $2 \pi / 3$-mode shots were confirmed in only the $4 \mathrm{~cm}$ and $8 \mathrm{~cm}$ wire cases (69 ns unprimed versus 29 ns and $49 \mathrm{~ns}$ for the $4 \mathrm{~cm}$ and $8 \mathrm{~cm}$ wire cases, respectively). 


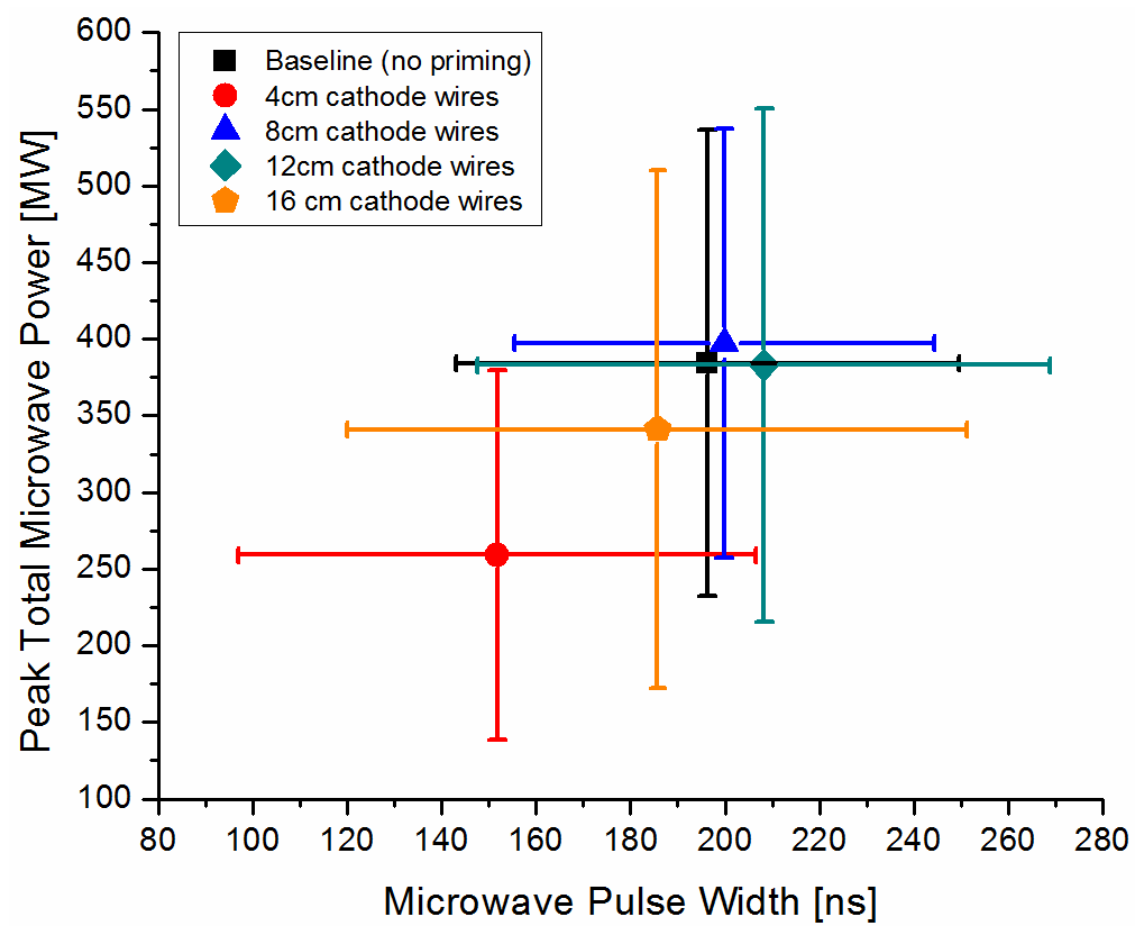

(a)

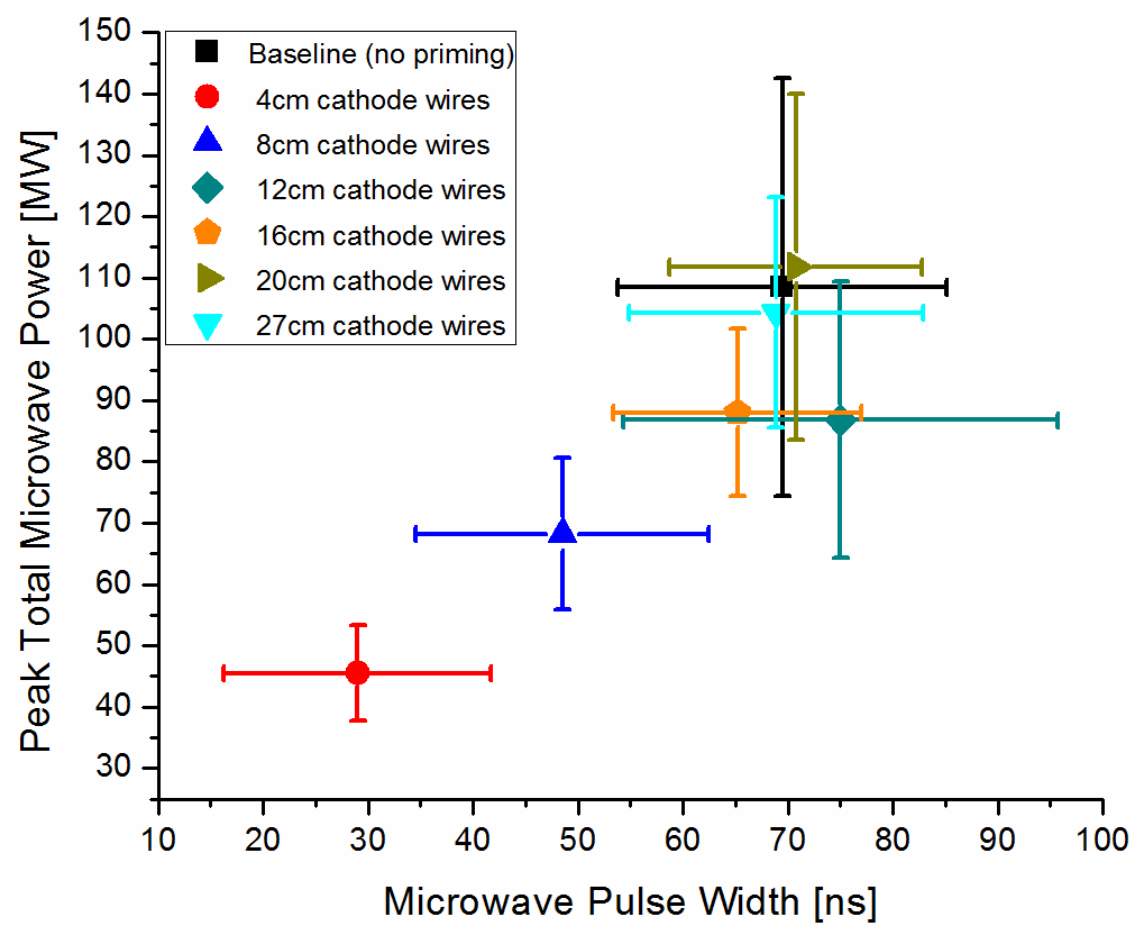

(b)

Figure 52. Mean values of peak total microwave power versus pulse width for the baseline and cathode wire cases for (a) $\pi$-mode shots and (b) $2 \pi / 3$-mode shots. 
Plots comparing microwave start-oscillation (starting) time and time to peak power are shown in Figure 53 (a) and Figure 53 (b), respectively. In these figures, microwave start-oscillation (starting) time is defined as the difference in time between the input voltage exceeding $15 \mathrm{kV}$ and the total microwave power exceeding $25 \mathrm{MW}$. Time to peak power is defined as the time between the input voltage exceeding $15 \mathrm{kV}$ and the time at which the total microwave power reaches the maximum value. When compared with the unprimed case, the $4 \mathrm{~cm}$, $8 \mathrm{~cm}$, and $12 \mathrm{~cm}$ wire cases showed statistically significant reductions in the time between the voltage turn on and the emission of microwave radiation from the magnetron, to a confidence level of 0.05 . Only the $4 \mathrm{~cm}$ and $8 \mathrm{~cm}$ cases showed statistically significant reductions in the time required for the magnetron to reach maximum microwave output power. 


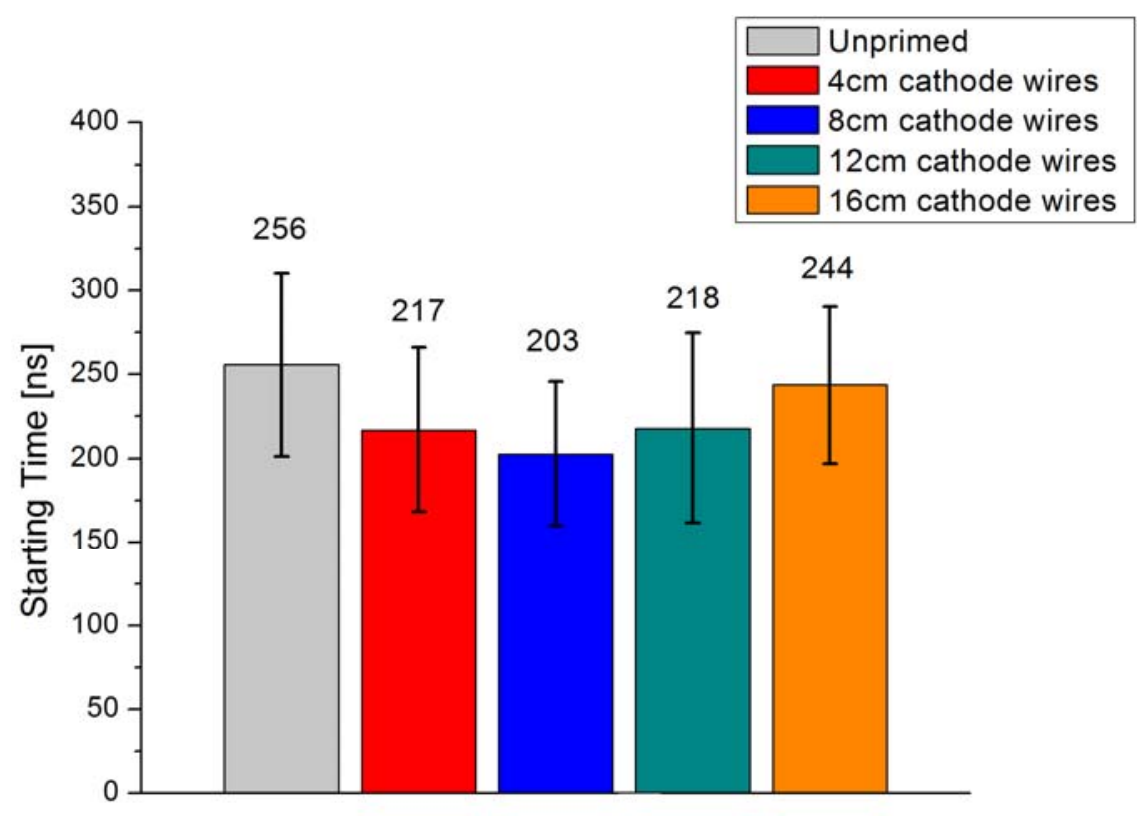

(a)

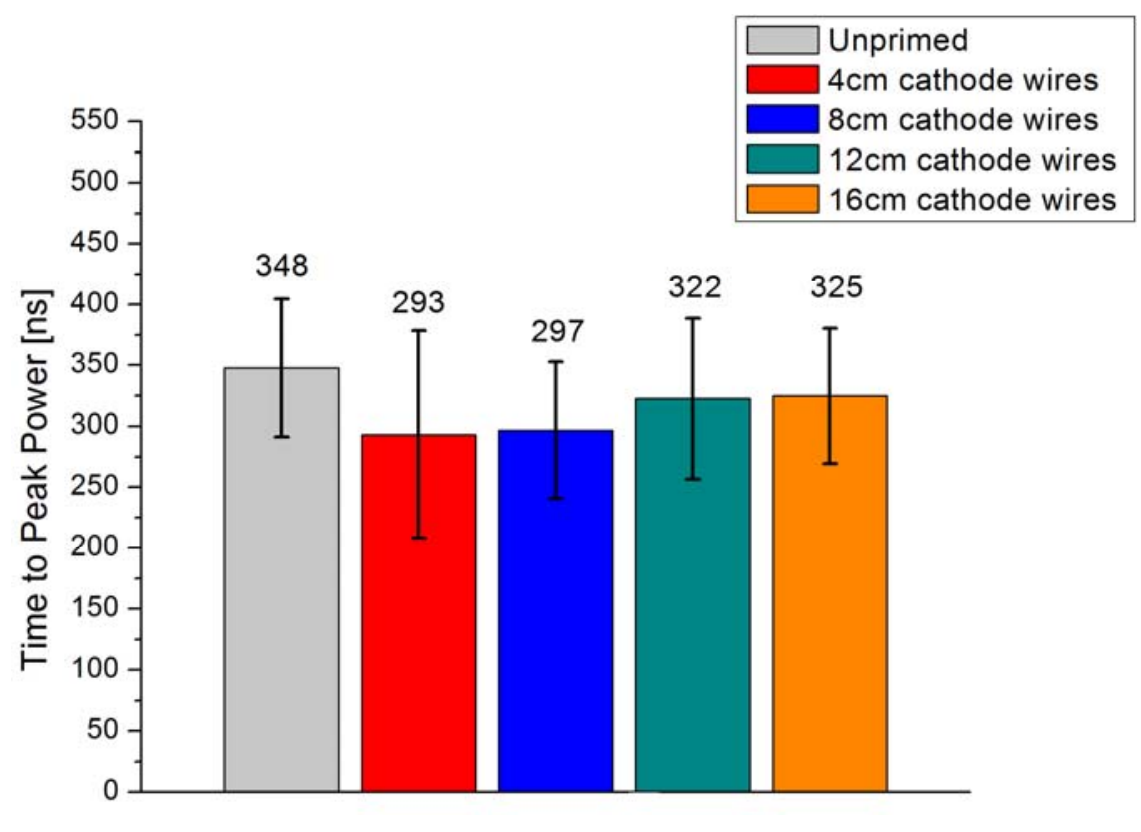

(b)

Figure 53. Mean values of magnetron $\pi$-mode oscillation starting time (a) and time to peak power (b) for the magnetically primed versus unprimed data sets. 
A summary of the starting current for the m-mode shots in the second experiment is displayed in Figure 54. Starting current is defined as the value of the magnetron entrance current at the time when the total microwave power exceeds $25 \mathrm{MW}$. With the exception of the $4 \mathrm{~cm}$ wire case, none of the mean starting current values for the $\pi$-mode shots of the magnetically primed cases were found to be statistically different than the mean of the baseline case. The 4 $\mathrm{cm}$ wire case was found to draw, on average, $7 \%$ more input current than the unprimed case at the time at which the magnetron started oscillating.

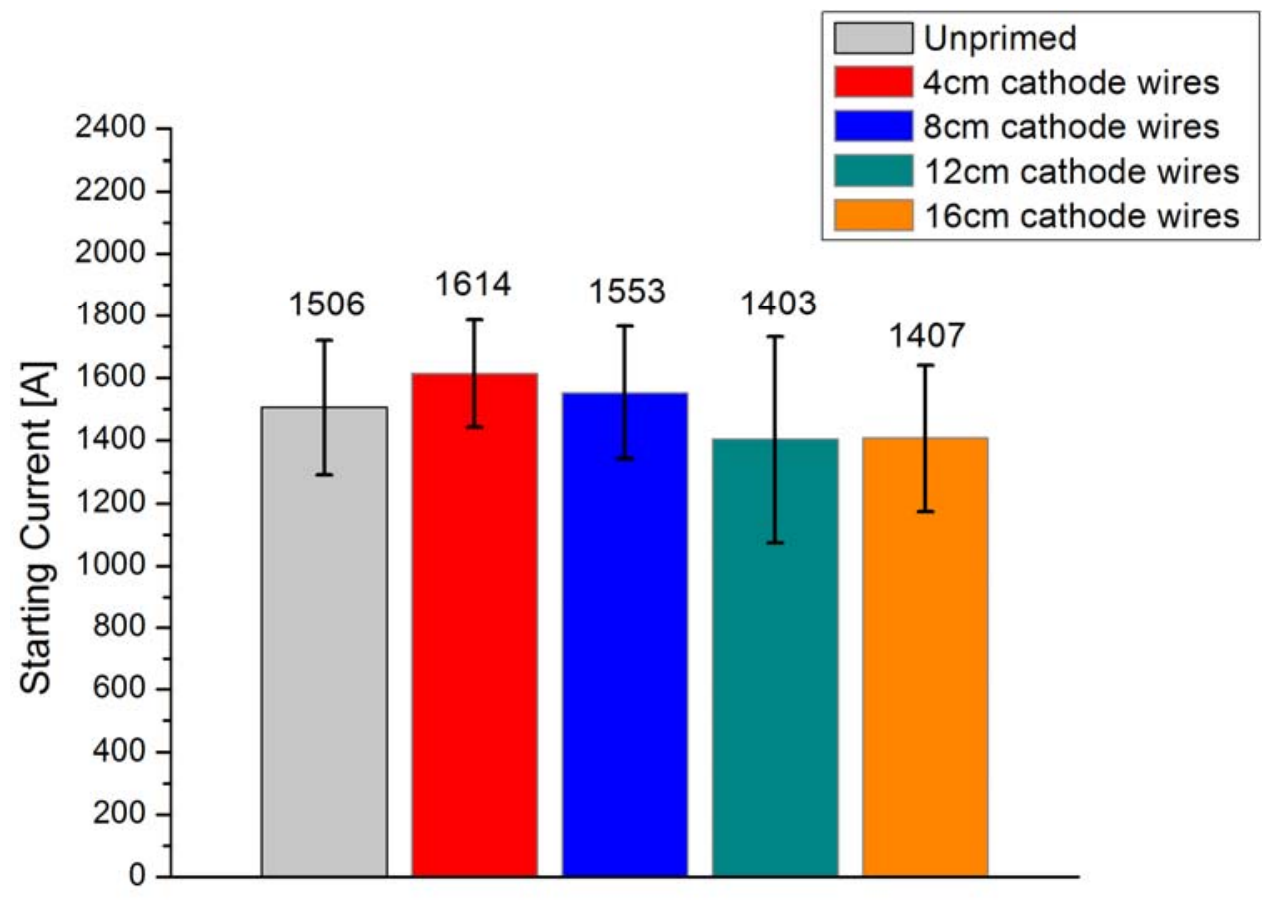

Figure 54. Magnetron starting current for $\pi$-mode shots in the magnetically primed versus unprimed cases.

Energy efficiency, defined as total energy contained in the magnetron microwave output divided by the total electrical input energy (input voltage multiplied by magnetron entrance current) from the start of the voltage pulse until 
the end of the microwave pulse, is displayed for the primed and unprimed cases in Figure 55. In the case of the $4 \mathrm{~cm}$ long cathode priming wires, the magnetron was found to operate at a reduced efficiency in the m-mode, when compared to unprimed $\pi$-mode operation (12\% primed versus $16 \%$ unprimed). $\pi$-mode operation in the other priming cases was shown to be statistically the same as the unprimed case to a confidence level of 0.05 , using a t-test.

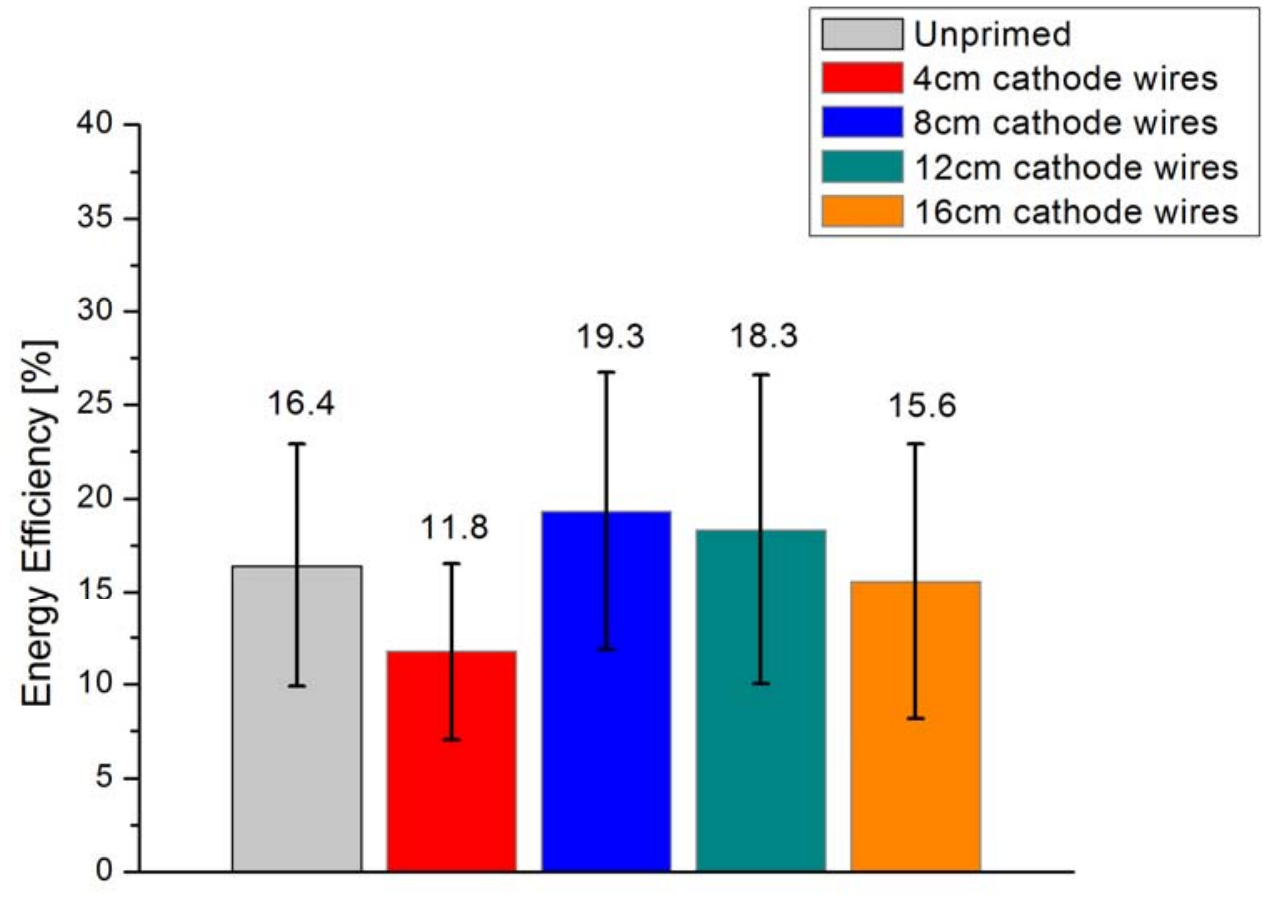

Figure 55. Magnetron energy efficiency for $\pi$-mode shots in the magnetically primed versus unprimed cases.

\subsubsection{Magnetic Priming at the Anode}

The third magnetic priming data set consisted of three magnetically primed cases, with $10 \mathrm{~cm}, 15 \mathrm{~cm}$, and $20 \mathrm{~cm}$ length sets of three $0.508 \mathrm{~cm}$ diameter high permeability wires, spaced 120 degrees apart and embedded in the cathode. The unprimed data set used as a control set for comparison is the 
same as used in the second magnetic priming experiment. The plots of Figure 56 (a)-(d) depict the voltage and magnetic field parameter space of the magnetically primed cases as well as the unprimed baseline set, in relation to the Buneman-Hartree resonance lines for the $\pi$ and $2 \pi / 3$ modes. Additional plots for frequency versus magnetic field are provided in Appendix C.

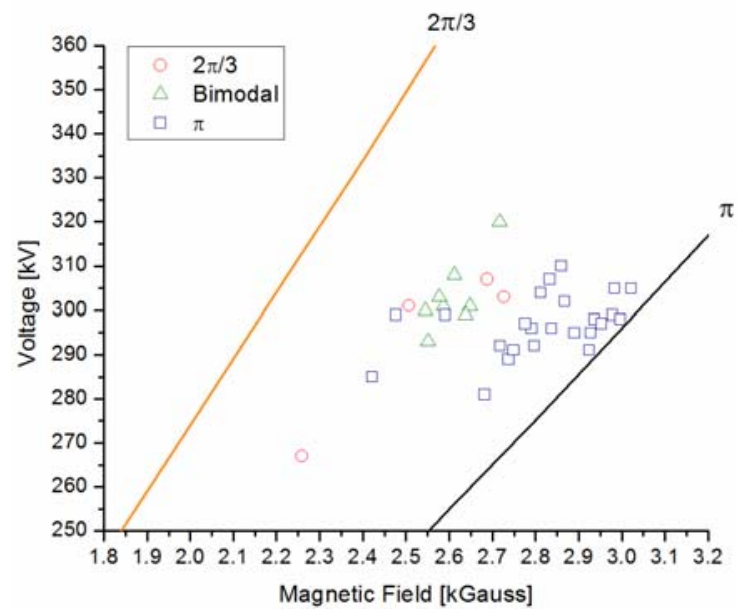

(a)

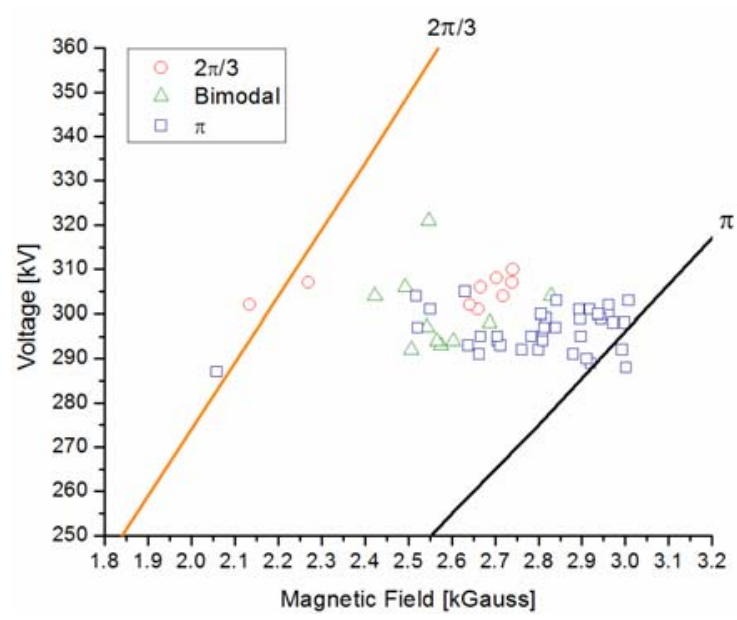

(c)

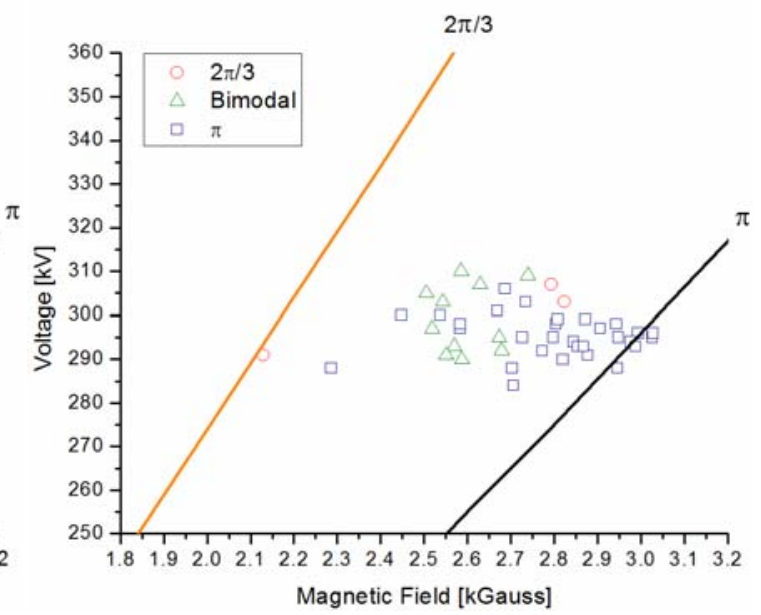

(b)

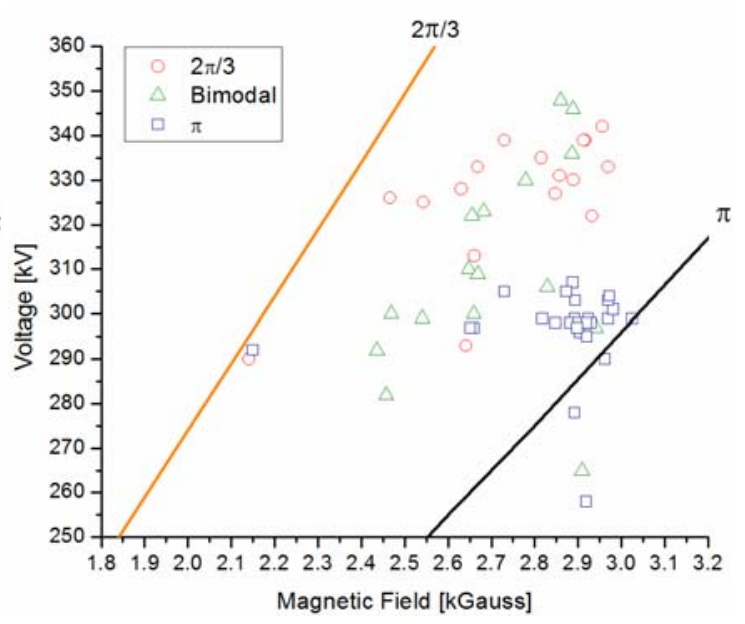

(d)

Figure 56. Experimental data points near the $2 \pi / 3$ and $\pi$-mode Buneman-Hartree resonance curves for the UM/L-3 Titan relativistic magnetron. (a) $10 \mathrm{~cm}$ anode wires, (b) 15 $\mathrm{cm}$ anode wires, (c) $20 \mathrm{~cm}$ anode wires, (d) unprimed (no wires) 
All of the anode wire cases showed an increase in the percentage of $\pi-$ mode shots, when compared to the baseline case $(65 \%, 69 \%$, and $67 \%$ for the $10 \mathrm{~cm}$ wire, $15 \mathrm{~cm}$ wire, and $20 \mathrm{~cm}$ wire cases, respectively, versus $45 \%$ for the unprimed case). Additionally, the anode wire cases all showed reductions in the percentage of pure $2 \pi / 3$-mode shots, when compared to the unprimed case.

The percentage of bimodal shots in both the primed and unprimed cases was found to be approximately the same.

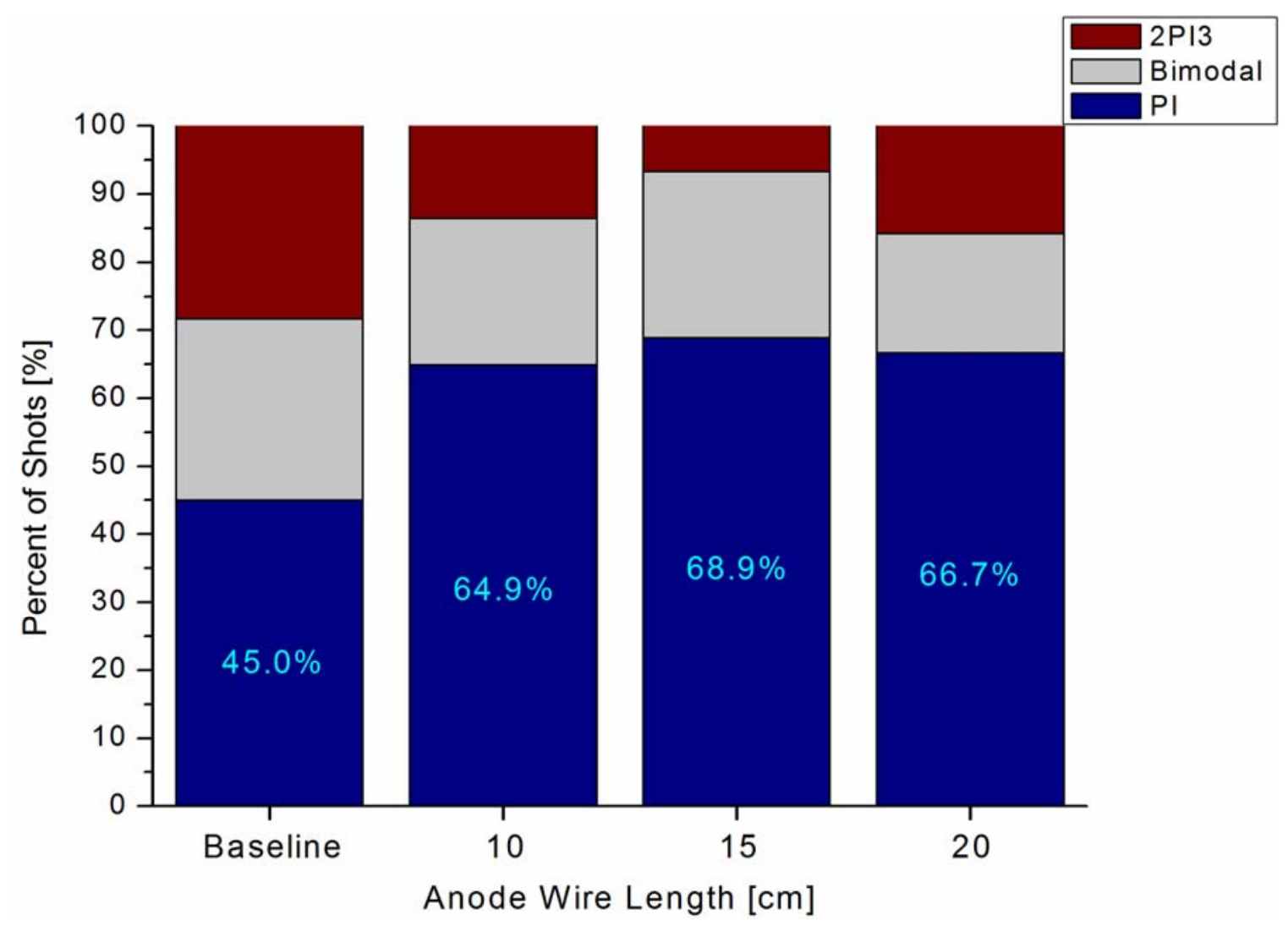

Figure 57. Shot percentages for the case of magnetic priming at the anode with balanced loading.

Figure 58 (a) displays mean peak microwave power versus mean pulse width for all $\pi$-mode shots observed in the third magnetic priming experiment. Mean peak microwave power versus mean pulse width is plotted for all $2 \pi / 3$ 
shots in Figure 58 (b). For the m-mode shots, only the $15 \mathrm{~cm}$ wire case was shown to have a statistically significant difference ( 0.05 confidence level) in either mean peak power or mean pulse width by a t-test. The mean peak power and mean pulse width values of the m-mode shots were both found to be lower in the $15 \mathrm{~cm}$ anode wire case than those of the baseline case (263 MW and $158 \mathrm{~ns}$ primed versus $416 \mathrm{MW}$ and $175 \mathrm{~ns}$ unprimed). All three magnetically primed cases showed statistically significant reductions in $2 \pi / 3$-mode shot mean peak power, compared to the unprimed case. While the $2 \pi / 3$-mode shots in the $10 \mathrm{~cm}$ wire and $15 \mathrm{~cm}$ wire cases showed significant reductions in mean microwave pulse widths from the baseline case, the $20 \mathrm{~cm}$ anode wire case was actually shown to increase the duration of the $2 \pi / 3$-mode shots over the baseline by about $56 \%$. 


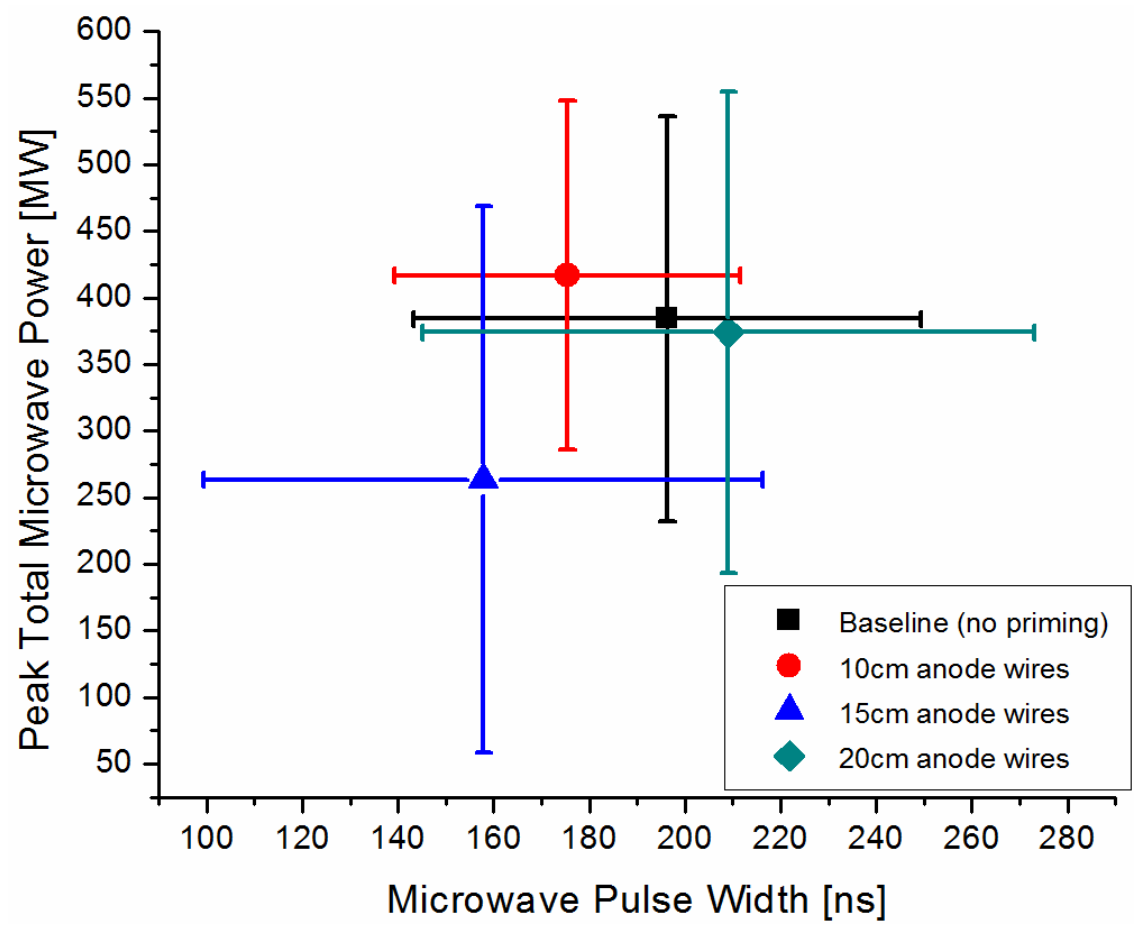

(a)

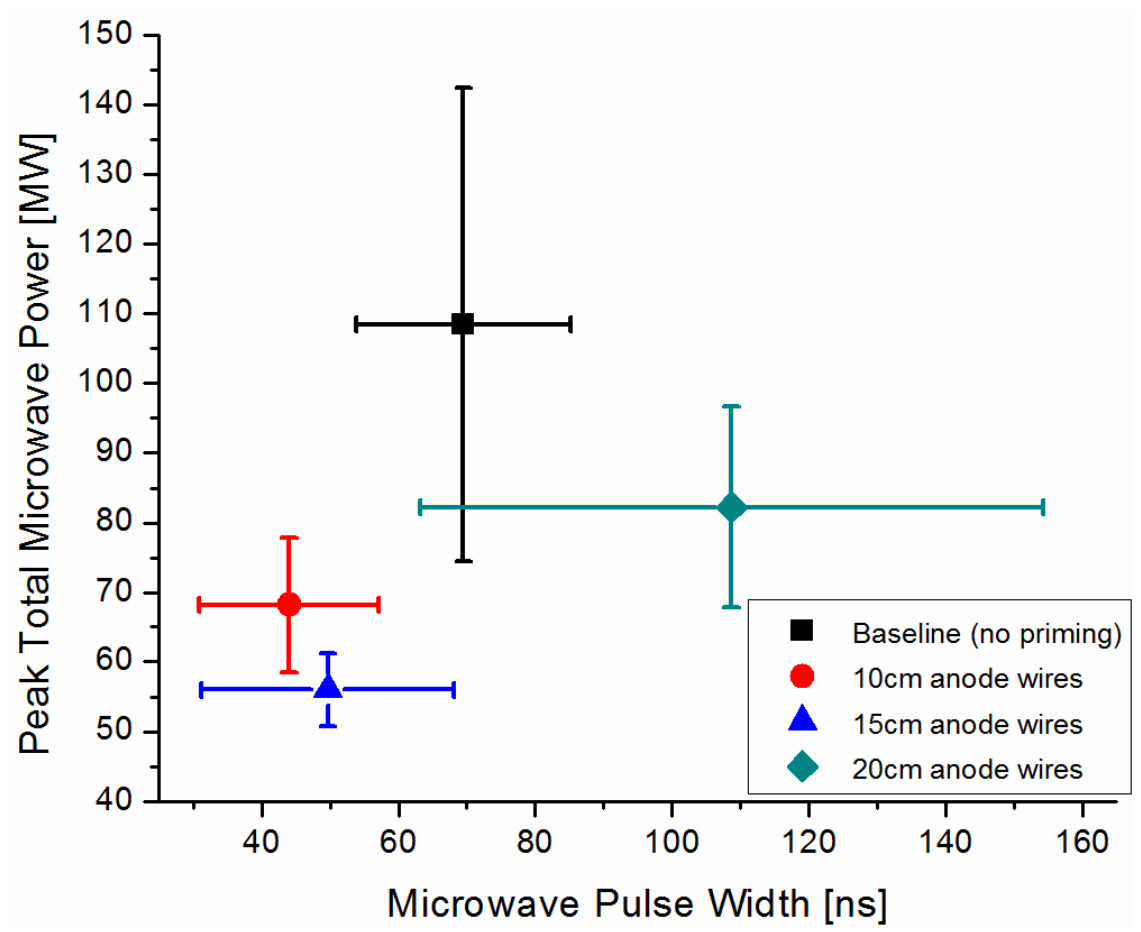

(b)

Figure 58. Mean values of peak total microwave power versus pulse width for the baseline and anode wire cases for (a) $\pi$-mode shots and (b) $2 \pi / 3$-mode shots. 
Microwave start-oscillation (starting) time and time to peak power comparisons for the primed and unprimed cases are shown in Figure 59 (a) and (b), respectively. All three magnetically primed at anode cases showed statistically significant reductions in starting time, compared to the unprimed case. Only the $10 \mathrm{~cm}$ wire and $15 \mathrm{~cm}$ wire cases, however, showed statistically significant reductions in time between voltage turn on and time at which maximum output power was achieved. 


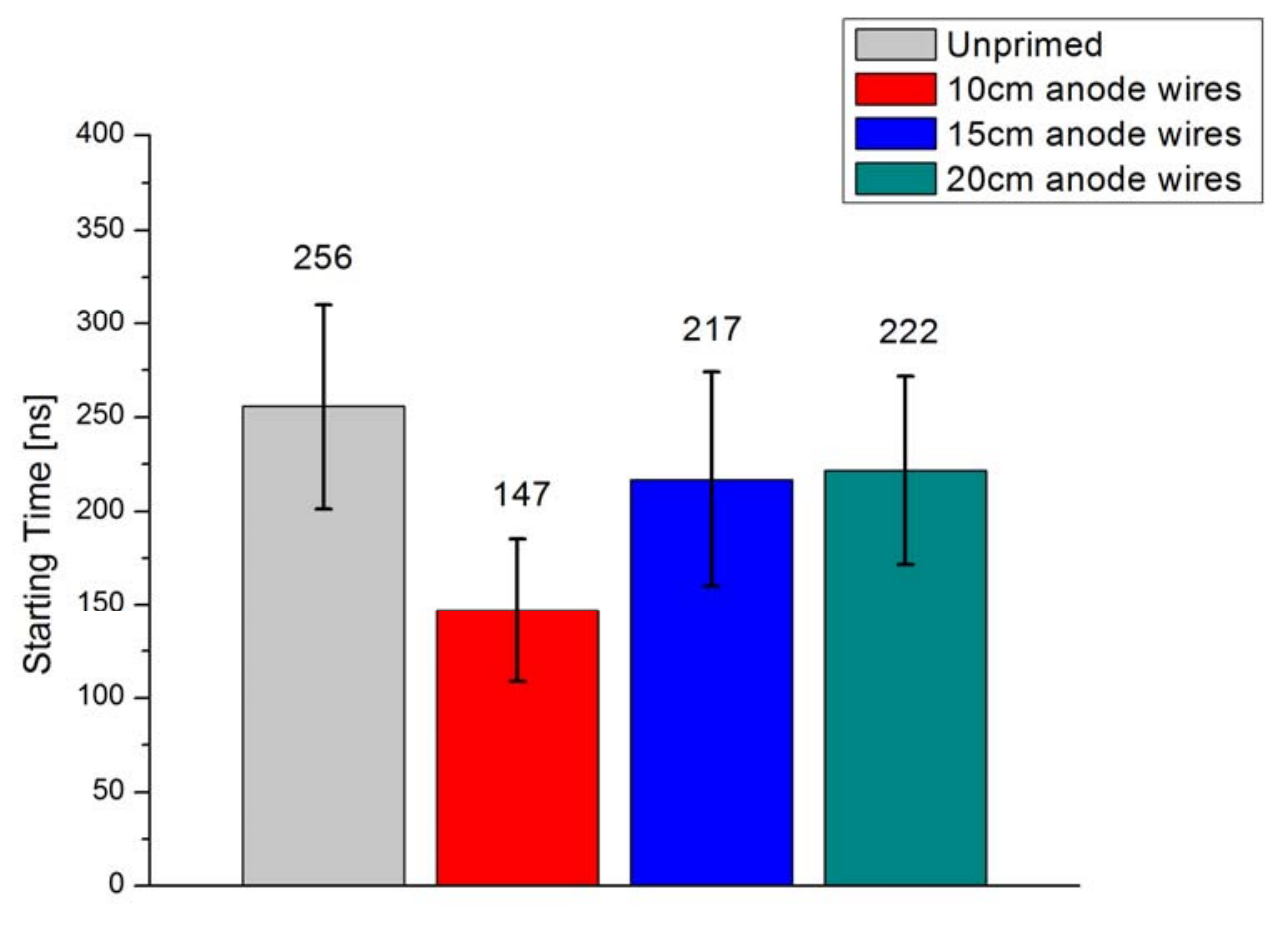

(a)

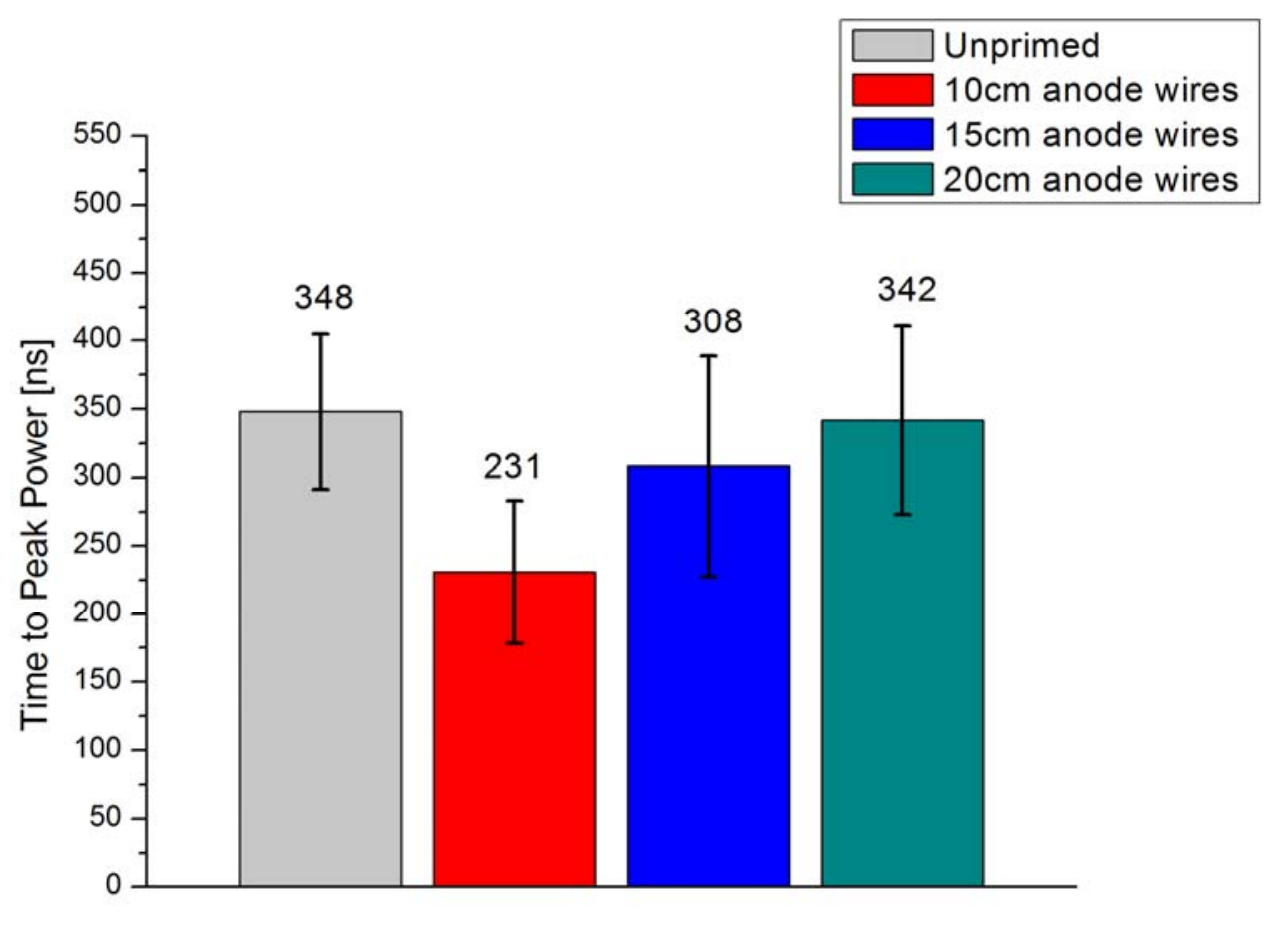

(b)

Figure 59. Mean values of magnetron $\pi$-mode oscillation starting time (a) and time to peak power (b) for the magnetically primed versus unprimed data sets. 
Magnetron start-oscillation current averages for $\pi$-mode shots for the three magnetically primed cases and the unprimed case are depicted in Figure 60. Differences in the starting cases between the cases could not be shown to be statistically different to a confidence level of 0.05 using a t-test.

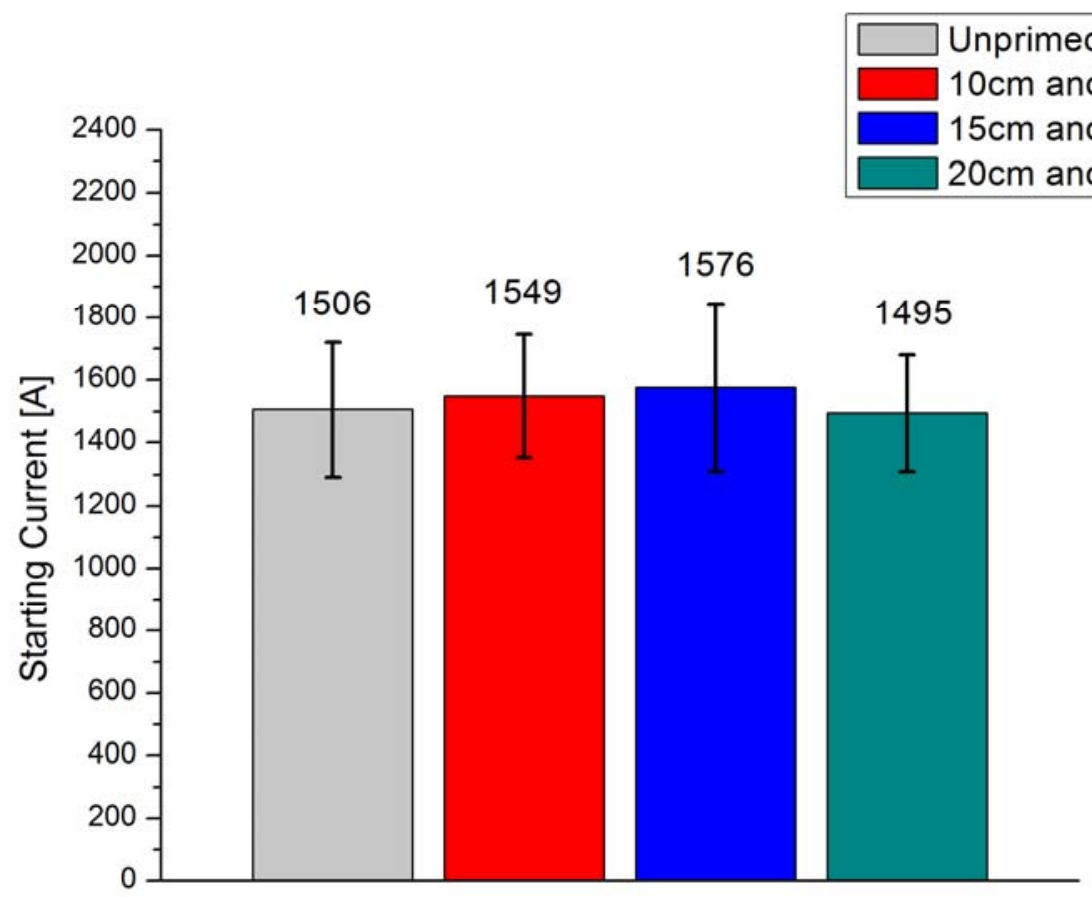

Figure 60. Magnetron starting current for $\pi$-mode shots in the magnetically primed versus unprimed cases.

Mean energy efficiency data for $\pi$-mode shots in the primed and unprimed cases are summarized in Figure 61. The m-mode energy efficiency of the $15 \mathrm{~cm}$ and $20 \mathrm{~cm}$ wire cases did not exhibit statistically significant differences from the energy efficiency of the unprimed baseline case. The $10 \mathrm{~cm}$ wire case did show an increase in energy efficiency of $5 \%$ over the unprimed case. 


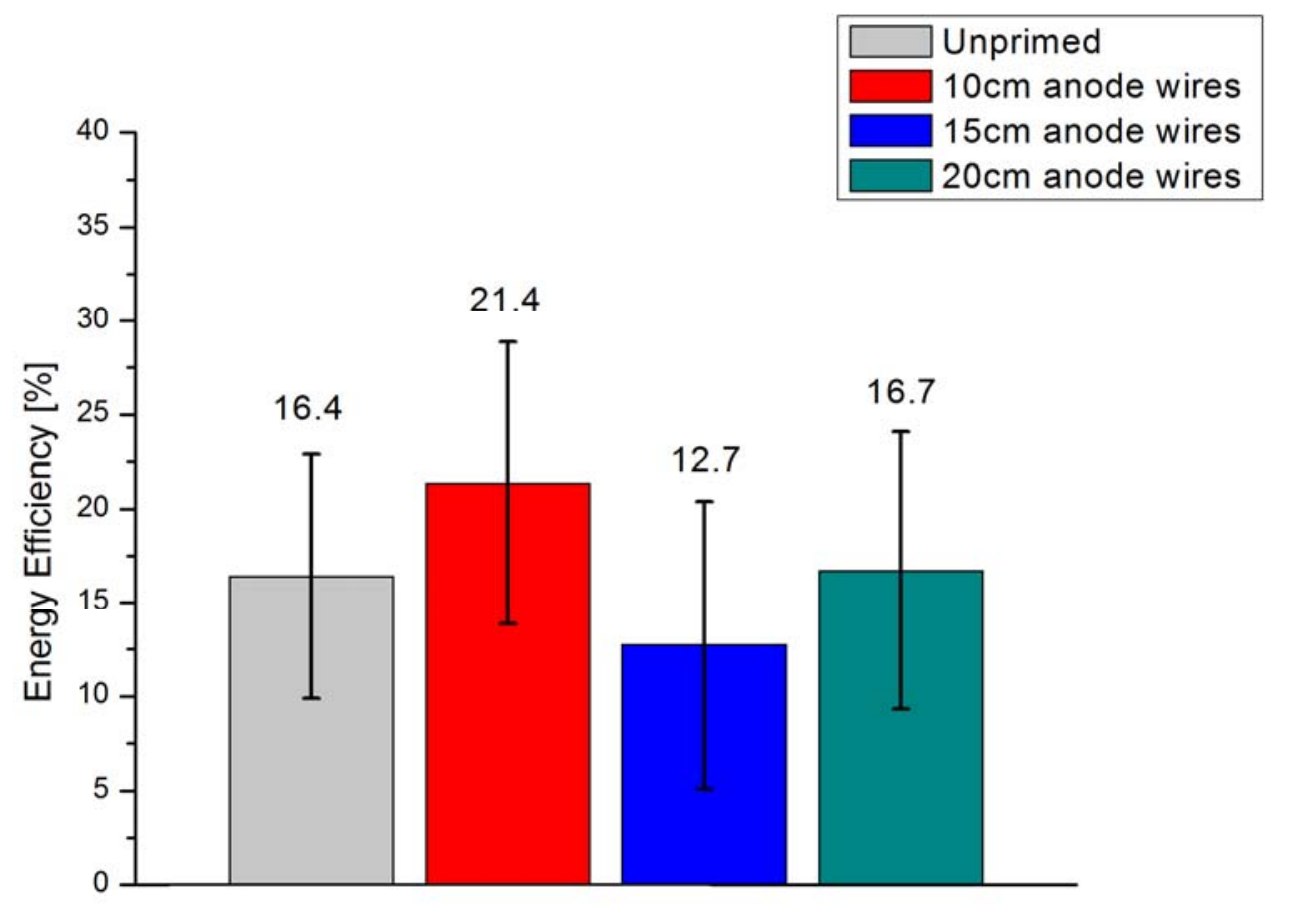

Figure 61. Magnetron energy efficiency for $\pi$-mode shots in the magnetically primed versus unprimed cases. 


\section{Chapter 5 \\ Magnetic Priming: Analysis, and Discussion}

Both the initial relativistic magnetron simulation research performed by

Jones and the latest magnetic priming research presented in Chapter 3 , involve a number of simplifications and assumptions that prevent direct quantitative comparisons from being made between the simulation results and the experiments [36]. Additionally, there was a substantial difference between magnetic priming amplitudes used in the simulations and those used in the experiments. The simulation magnetic perturbations were on the order of $30 \%$ of the magnitude of the unprimed magnetic field. Pulsed magnetic field measurements performed in the experimental configuration indicated magnetic perturbation amplitudes that were approximately an order of magnitude less than the simulation perturbation amplitudes. Other differences include cathode size (larger cathodes used in the simulations to reduce simulation run-time), applied voltage waveform (in the simulations voltage was ramped to desired value over a few RF cycles, while the voltage from MELBA-C required approximately $100 \mathrm{~ns}$ to reach max value), and idealized magnetic priming scheme (applicable only to Jones' simulation research). 
Although quantitative comparisons are not valid, qualitatively, the magnetic priming experiments show many of the same trends demonstrated in the magnetically primed simulation magnetron. The most notable effect observed both in Jones' simulations and in the experiments was that magnetic priming enhanced the degree of mode control in the relativistic magnetron. The best case results for the three experimental campaigns show increases in $\pi-$ mode shots by as much as $30 \%$ ( $4 \mathrm{~cm}$ long wires, balanced loading case) over the unprimed baseline case.

As stated previously, mode control was not tested in the realistic 3-D geometry magnetic priming simulations; however, it was noted that in these simulations, the magnetic priming at the cathode cases showed the best overall performance with the shortest wire set $(4 \mathrm{~cm}$ long wires). The simulation magnetron performance was also noted to grow progressively worse as the wire lengths were increased, to the eventual point of failure to start up. This trend was observed in the mode control effects of the magnetic priming wires used in the second experiment (magnetic priming at the cathode with balanced loading). The best overall mode control was demonstrated in the $4 \mathrm{~cm}$ and $8 \mathrm{~cm}$-long wire cases. The $12 \mathrm{~cm}$ and $16 \mathrm{~cm}$-long wires showed little or no enhancement of $\pi-$ mode. Although the longest wires cases, $20 \mathrm{~cm}$ and $27 \mathrm{~cm}$, did not prevent oscillation of the magnetron, they did cause the m-mode oscillations to be nearly completely suppressed.

In the simulations, excessive magnetic priming wire length moved the zones of high $\mathrm{B}_{r}$ and $\mathrm{B}_{\theta}$ perturbations away from the emission region of the 
cathode and also caused an average suppression of the $B_{Z}$ magnetic field around the cathode in a region long enough to cover both electrostatic end hats. This effect allowed large amounts of current to escape around the end hats, as shown in Figure 31. The excessive end loss current prevented the start of oscillation in the simulation magnetron. Increasing the priming wire length in the experiment also served to move the expected zones of higher $B_{r}$ and $B_{\theta}$ perturbations out of the cathode's $10 \mathrm{~cm}$-long emission region. Because the perturbation strengths of the wires in the experiment were much lower than were the simulated wires, the excessive end loss current effects were not observed. A milder reduction in the average $B_{Z}$ field around the cathode would, however, be expected to shift the Buneman-Hartree resonance conditions in favor of the $2 \pi / 3$ mode, which was observed in the $20 \mathrm{~cm}$ and $27 \mathrm{~cm}$ wire cases.

The relation of the mode control effects observed in the experimental magnetic priming at the anode cases to the performance of the 3-D anode wire simulations is less clear than the correlation between the simulation and experimental cathode wire cases. All three experimental anode wire cases showed a $20 \%$ increase in $\pi$-mode shots over the baseline case. The simulation wire length cases and the experimental wire length cases overlap for only the 10 $\mathrm{cm}$ wires. The simulated $10 \mathrm{~cm}$ wire case did show some positive effects of magnetic priming (faster startup) but also showed an increase in end loss current and a correspondingly large decrease in power, compared with the baseline case. The anode wire cases more closely resemble the experimental configurations used by Neculaes for the kW oven magnetrons $[10-15,19]$. 
Another important magnetic priming effect observed in both Jones' simulations and the 3-D simulations presented in Chapter 3 was rapid growth of the $\pi$-mode. In the 3-D simulation cases, all of the cathode wire simulations that started oscillating showed faster $\pi$-mode growth, even in cases where the magnetron oscillation eventually died out due to excessive end loss current. In the anode wire simulations, the $8 \mathrm{~cm}$ and $10 \mathrm{~cm}$ long wire cases showed more rapid $\pi$-mode growth than the unprimed case; however, the two shortest anode wire cases $(4 \mathrm{~cm}$ and $6 \mathrm{~cm})$ did not demonstrate any change in the $\pi$-mode growth rate, when compared to the unprimed case. With one exception, all of the experimental magnetic priming cases that demonstrated increased mode control over the unprimed case also demonstrated significant reductions in time to startoscillation and time to peak microwave power. The $20 \mathrm{~cm}$ anode wire case, although showing an increase in $\pi$-mode shots of $20 \%$ over the baseline case, only yielded reductions in start-oscillation time, not in time to peak microwave power.

Neculaes' previous work involving the magnetic priming of kilowatt oven magnetrons $[10-15,19]$ demonstrated reductions in the starting current for the $\pi-$ mode, when compared with the unprimed case. Reductions in starting current were observed in the magnetically primed $\pi$-mode shots of the relativistic magnetron experiments in the first experiment (magnetic priming at the cathode with unbalanced loading). The second and third experiments (magnetic priming at the cathode and anode, respectively, with balanced loading) showed no statistically significant reductions in starting current from the baseline case. 
Another finding in Neculaes' oven magnetron priming research was a modest reduction in steady-state $\pi$-mode output power $(10 \%-20 \%)$ in the magnetically primed case when compared to the unprimed case. These reductions in m-mode power and efficiency were also noted in all of the 3-D simulations performed. In the second and third experiments, reductions in power were only observed in the $4 \mathrm{~cm}$ cathode wire case and the $15 \mathrm{~cm}$ anode wire case. All other cases showed peak power outputs with no statistically significant difference from the unprimed case. In contrast, the first magnetic priming experiment (4 cm long wires, unbalanced loading), demonstrated a twofold increase in the peak microwave power in the primed case over the unprimed case.

Some caution is required when comparing the steady-state, magnetically primed power outputs of the $\mathrm{kW}$ oven magnetrons and simulation relativistic magnetrons to the peak power outputs of the primed relativistic magnetron. Due to the pulsed mode operation of the relativistic magnetron, in combination with other microwave pulse shortening effects observed in relativistic magnetrons $[7,60-62]$, it can be argued that "steady state" power output is never reached. Additionally, oven magnetrons used by Neculaes were "locked" into the m-mode in both the primed and unprimed cases (with a consistent center frequency for the microwave output). The reduction in efficiency in the oven magnetron experiments $[10-15,19]$ was likely due to a reduction in the overall magnetic field. This effect was also observed in the high fidelity particle-in-cell oven magnetron simulations performed by Luginsland et al. [21]. 
The relativistic magnetron has a significant shot-to-shot frequency variation, as shown in Figure 45 and implied by Figure 49 and Figure 56 . Even in relativistic magnetron shots that are clearly within the m-mode regime, varying degrees of mode competition and beam loading can still be present (and likely contribute to the frequency variation). These additional mode contributions have been observed in 3-D simulations of the UM/L-3 relativistic magnetron using the Air Force Research Laboratory's ICEPIC particle-in-cell code [63-67]. These ICEPIC simulations incorporated a magnetron model that more closely matches the UM/L-3 magnetron experiment. The ICEPIC model included an accurate representation of the waveguide extraction and microwave loading geometry present in the experiment that was not included in the 3-D magnetic priming simulations performed using MAGIC PIC and presented in Chapter 3.

The difference in the power trends observed in the unbalanced loading case and those observed in all other balanced loading primed simulations and experiments could partly be related to the effects of the asymmetric loading on the baseline m-mode operation. It should be emphasized that the overall perwaveguide power outputs observed in the unbalanced loading case were substantially lower than that in the second and third experiments (magnetic priming at the cathode and anode, respectively, with balanced loading). It is possible that power-related aspects of $\pi$-mode operation in the non-ideal operating conditions created by the asymmetric loading may be more easily influenced by magnetic priming at the cathode than m-mode operation in a balanced-loading configuration. Another possible cause for the observed 
increase in power of the magnetically primed case in the first experiment that must be mentioned in light of Ref. [37] is related to microwave window breakdown. The issue of microwave window breakdown, which affects only the first experimental campaign, will be discussed more fully in Chapter 6 . 


\section{Chapter 6 \\ Dielectric Microwave Window Breakdown}

In the course of performing HPM experiments on the UM/L-3 Titan relativistic magnetron it was discovered that flashover was occurring on the vacuum side of the microwave windows nearly every magnetron shot when the windows were located $3 \mathrm{~cm}$ from the microwave output coupling aperture. When the window was moved an additional 30 centimeters further away from the magnetron aperture, window breakdown events ceased, even though the magnetron operating conditions, and therefore generated microwave power, remained the same. It is important to note that a large body of work already exists on theoretical [68-74], simulation $[65,69,72,75,76]$, and experimental [7784] studies of breakdown and flashover mechanisms on dielectric microwave windows. In this chapter, we utilize 3-D Electromagnetic particle in cell (PIC) computer simulations to focus on the investigation of likely physical mechanisms for the aforementioned change in behavior of the microwave windows in these two configurations. 


\subsection{Experimental Configuration}

The UM/L-3 Titan relativistic magnetron (described previously in Chapter 4 ) is designed with six coupling cavities in the anode block vacuum housing. Each $3 \mathrm{~cm}$ deep coupling cavity is separated from its corresponding anode cavity by a $10.6 \mathrm{~cm}$ long, $1.75 \mathrm{~cm}$ wide aperture cut in the back wall of the anode. This geometry allows microwave radiation to directly couple out of any or all of the six resonator cavities.

In the original microwave window configuration, three WR650 output waveguides were attached to alternate cavities. The remaining cavities were sealed off with flat stainless steel plates. Polymethyl methacrylate (also known as PMMA or Lucite) windows, $1.27 \mathrm{~cm}$ thick, were used at the vacuum barrier between the magnetron and the waveguides. The waveguide section between the microwave windows and their corresponding loads were filled with sulfur hexafluoride gas at atmospheric pressure. Due to the positioning of the windows within the coils, the magnetic field at the window surface was approximately the same magnitude and polarity as the magnetic field in the magnetron itself.

Figure $62(a)$ is a schematic of the original experimental configuration, including magnetron housing, output waveguides, magnet coils and a number of diagnostic connections. Figure 62 (b) is a simplified schematic showing microwave window positioning in relation to the magnetron and power diagnostics. 


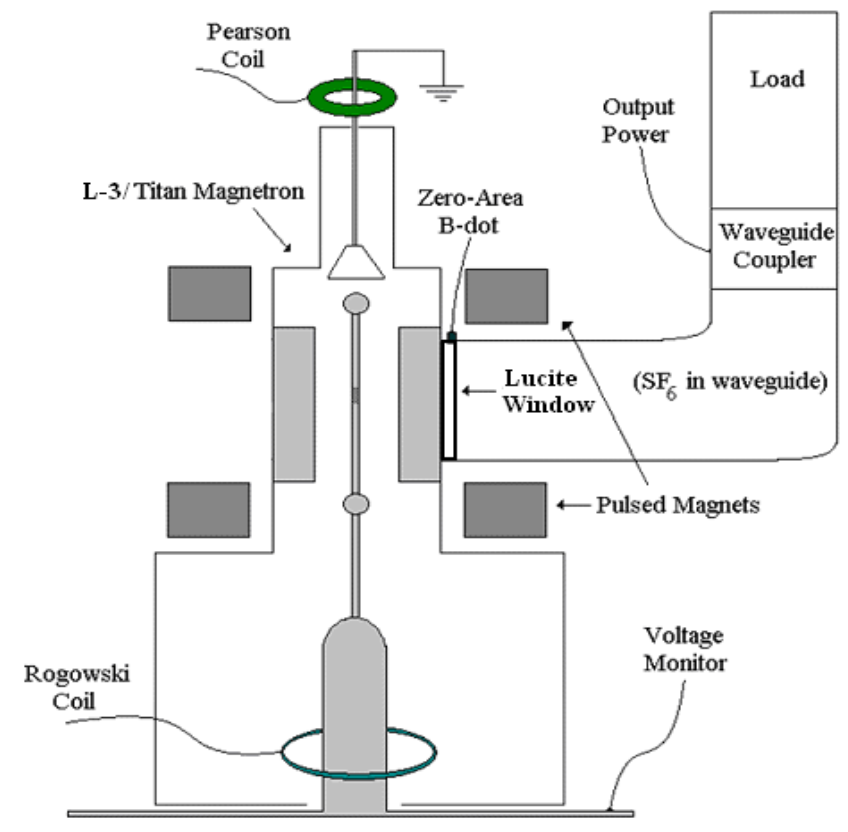

(a)

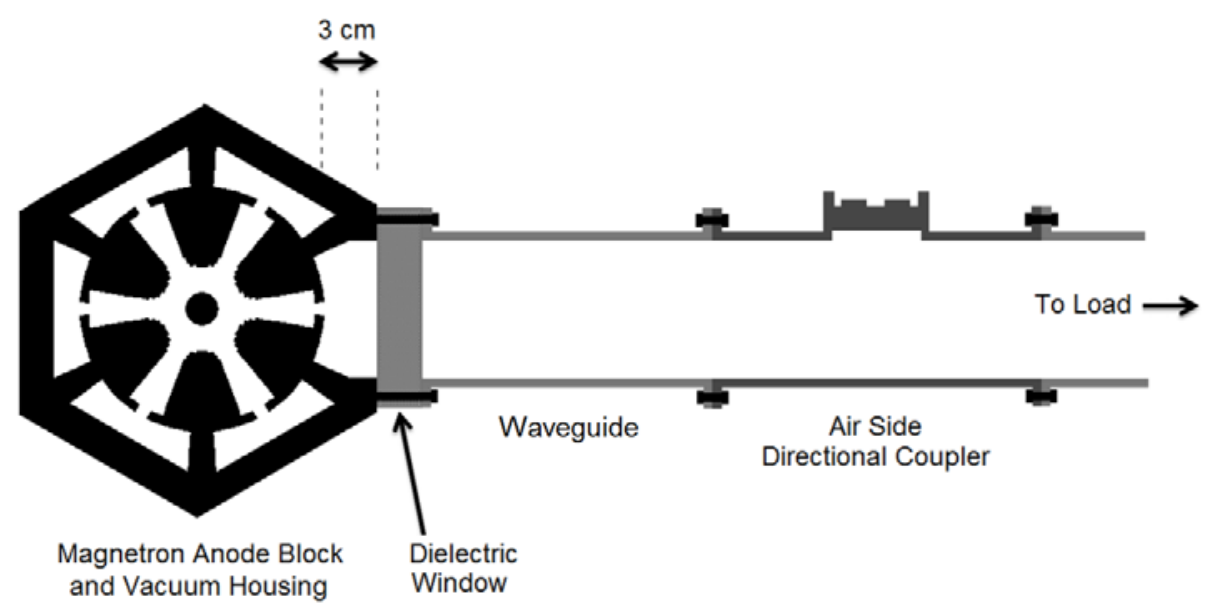

(b)

Figure 62. Schematic diagram of the UM/L-3 Titan relativistic magnetron experiment in the original configuration. (a) Approximate locations of the experiment diagnostics (reproduced from Ref. [31]). (b) Dielectric window positioning.

Electrical contact between the magnetron housing and the waveguides was maintained by the ten metal bolts threaded through the outer rim of the 
microwave window. Figure 63 shows a new Lucite window with bolt holes bored through the edges.

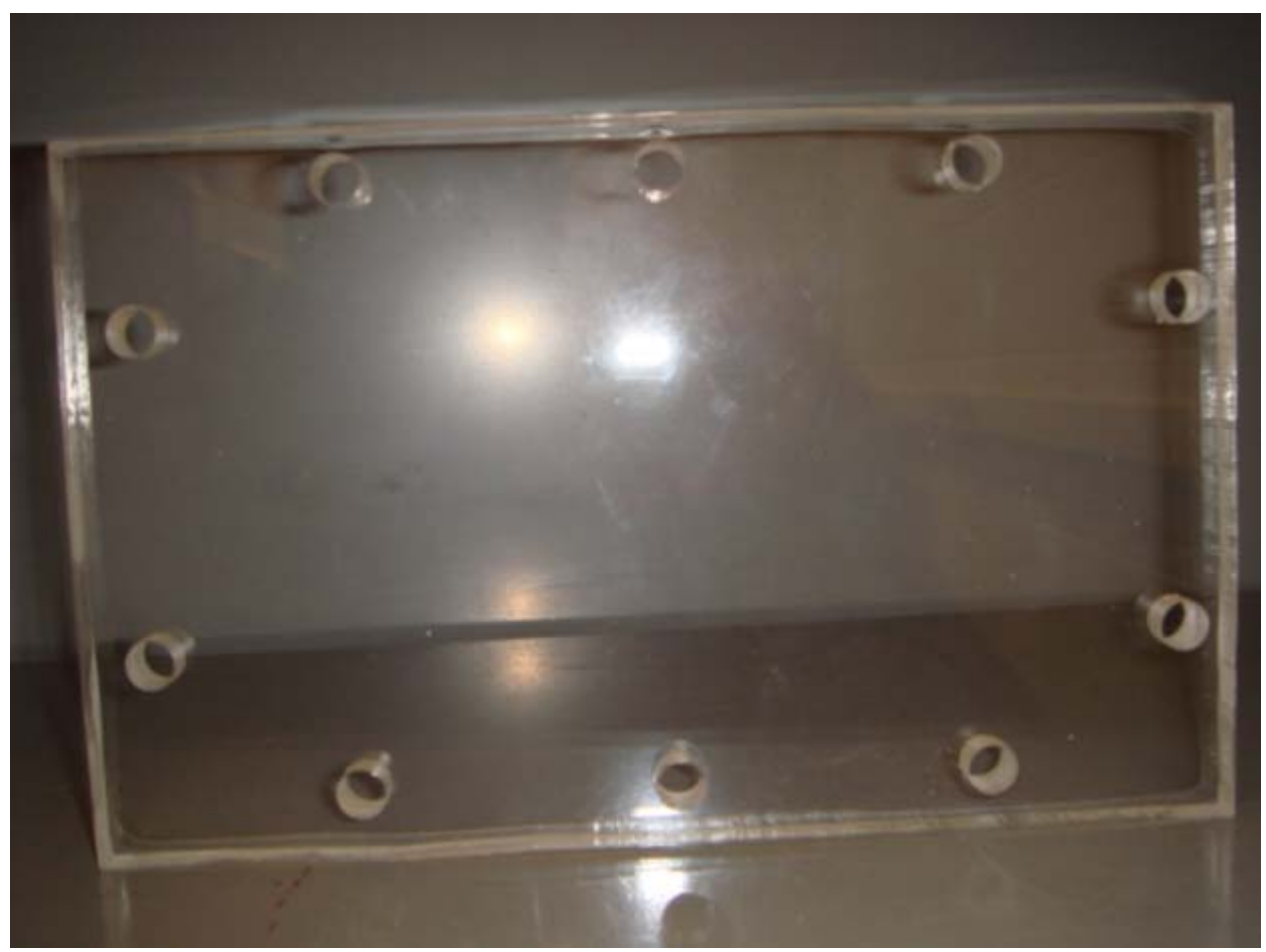

Figure 63. Lucite window used in the original microwave window configuration. Window dimensions are $22 \mathrm{~cm} \times 14 \mathrm{~cm} \times 1.27 \mathrm{~cm}$.

In the new, upgraded window configuration, three vacuum-rated directional coupler waveguide segments, designed by M. D. Haworth and built by the Air Force Research Laboratory, were mounted between the coupling cavities and the microwave windows. The inclusion of the vacuum side power couplers moved the microwave windows an additional $30 \mathrm{~cm}$ away from the anode apertures, as shown in Figure 64 (a). Figure 64 (b) depicts a simplified schematic of the new window positioning in relation to the magnetron and power couplers. In their new locations, the windows were far enough away from the 
coils for the magnetic field at the windows to be considered negligible. The waveguide section downstream of the windows was again filled with sulfur hexafluoride at atmospheric pressure.

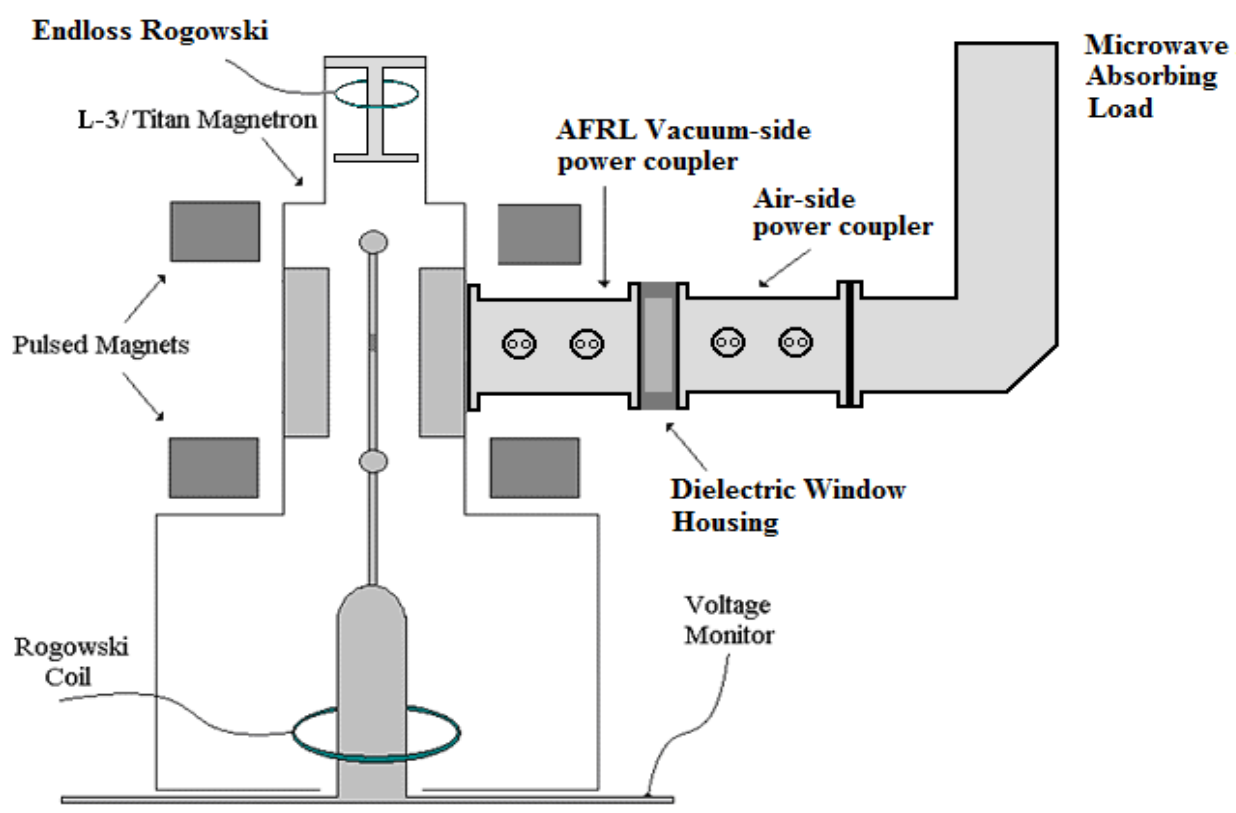

(a)

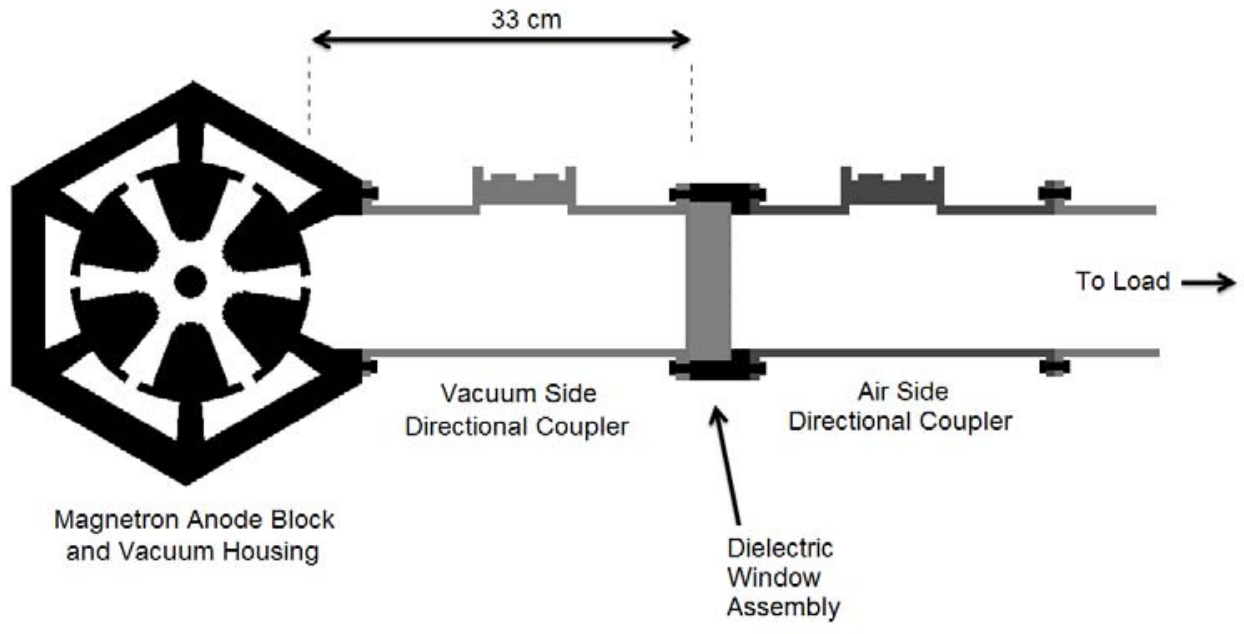

(b)

Figure 64. Schematic diagram of the UM/L-3 Titan relativistic magnetron experiment in the new upgraded configuration. (a) Approximate locations of the experiment diagnostics. (b) Dielectric window positioning. 
In addition to adding the vacuum side power couplers, the microwave window mounts were redesigned to better maintain waveguide continuity in the region around the microwave windows. One of the redesigned windows and mounting frames, prior to installation, is shown in Figure 65.

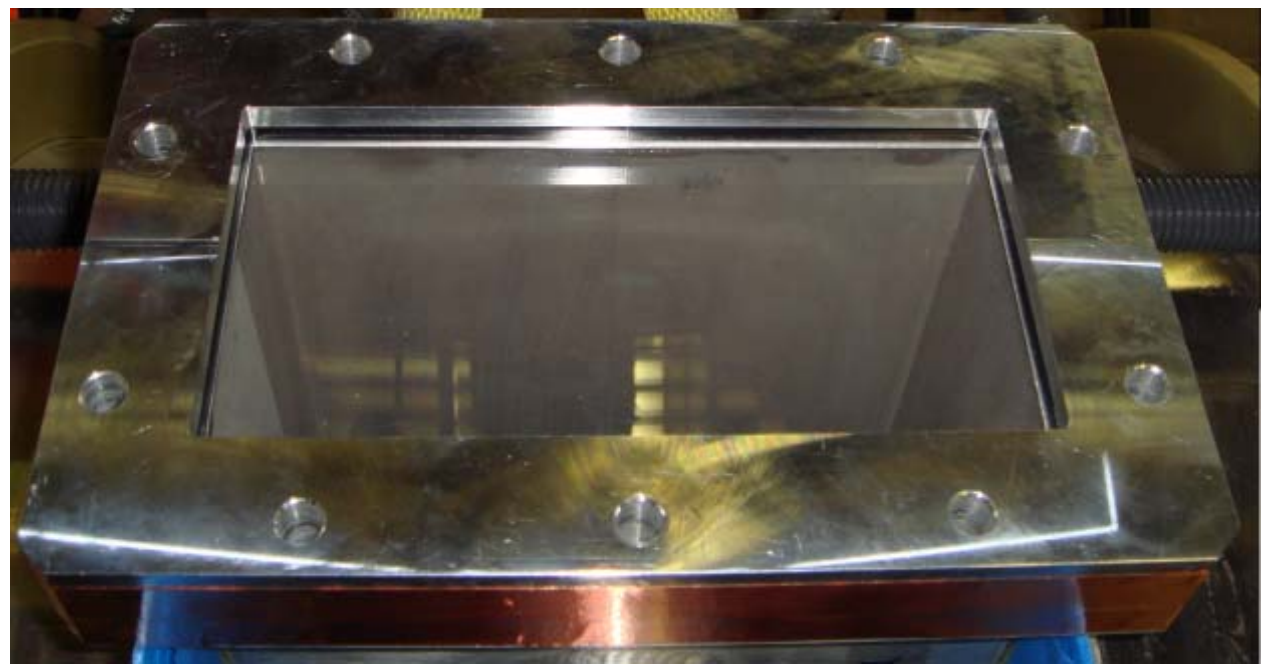

Figure 65. Uninstalled Upgraded Microwave Window

\subsection{Experimental Results}

In the original configuration, microwave pulse widths were found to average $135 \mathrm{~ns}$. Microwave signals from the directional power couplers were observed to cut off abruptly, only reaching single waveguide output powers of approximately $35 \mathrm{MW}$. Figure 66 (a) and (b) depict microwave signal data for magnetron shot 12251 . In this shot, the magnetron was oscillating in the $2 \mathrm{pi} / 3-$ mode, but the microwave signal peak power and pulse width are representative for both pi and 2pi/3 mode shots in the original window configuration. 


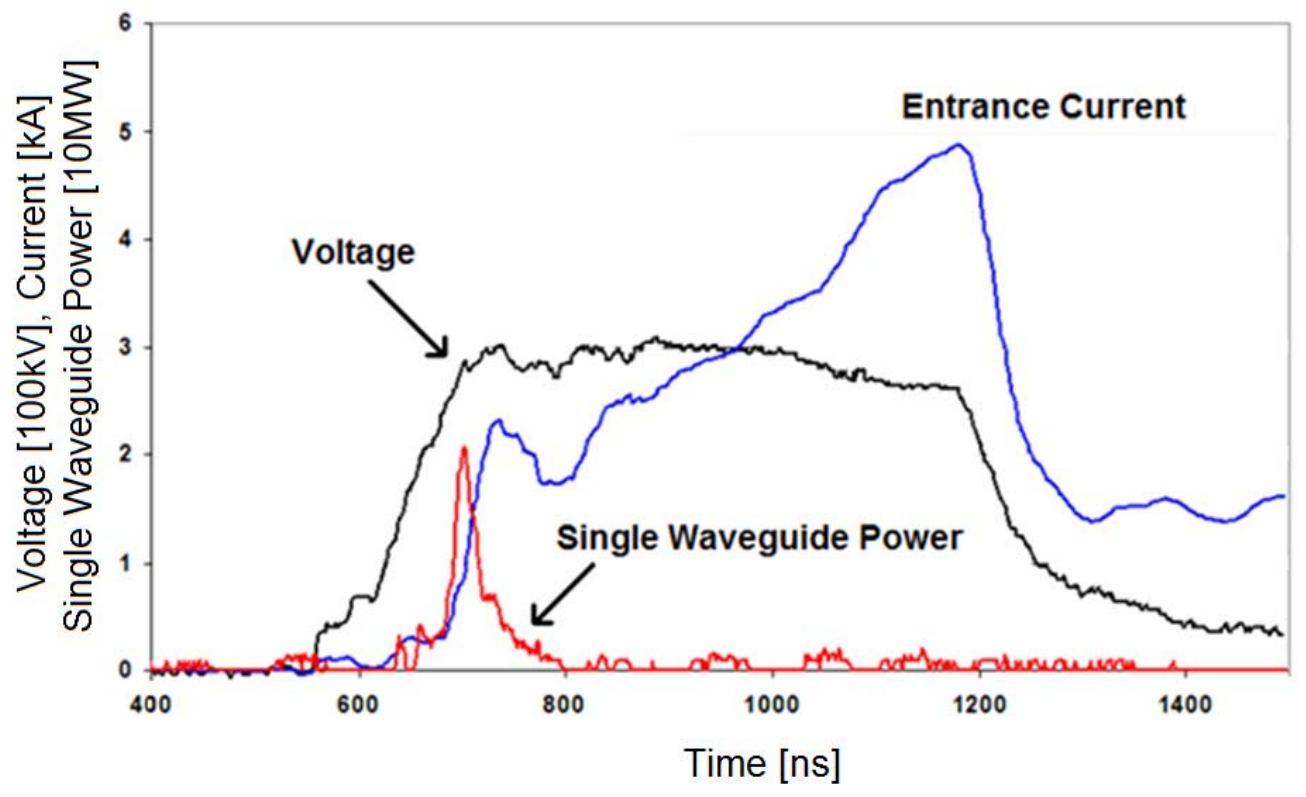

(a)
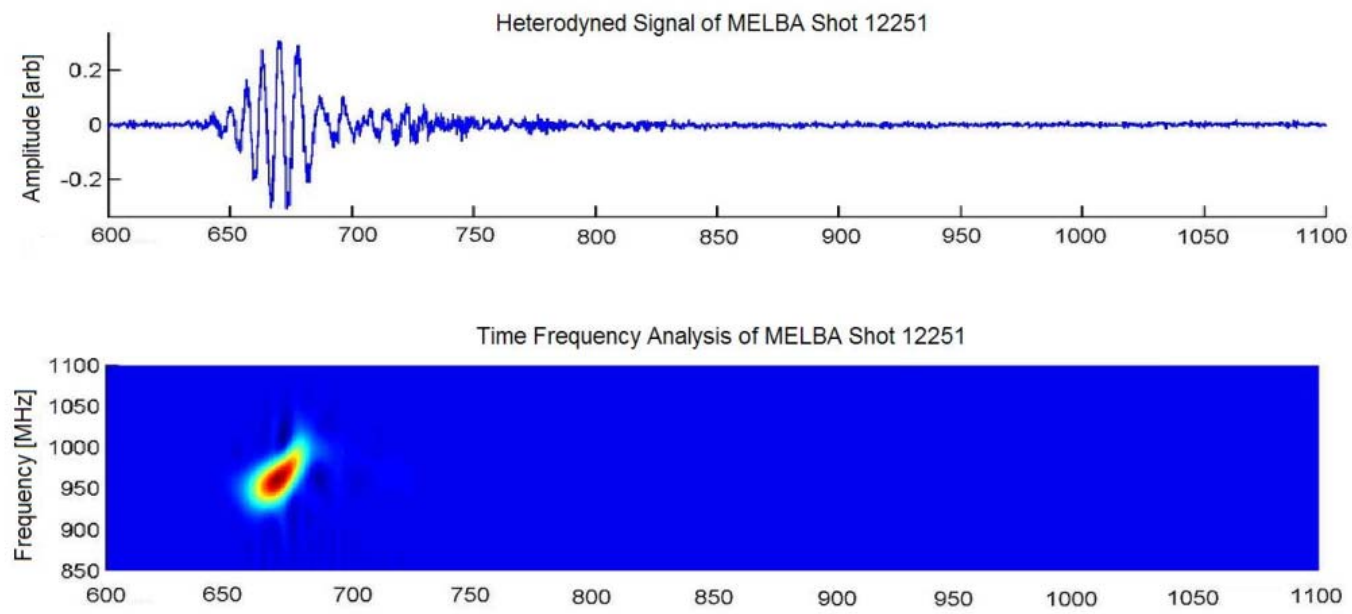

(b)

Figure 66. Relativistic magnetron performance in the original configuration (magnetron shot 12251). (a) Accelerator voltage, entrance current, and single waveguide microwave power. (b) Heterodyned signal and time frequency analysis of the microwave signal.

Because the microwave windows were located between the magnetron and the directional power couplers, window flashover plasma was suspected to be the cause of the abrupt microwave signal cutoff. To help confirm this 
suspicion, an arc detector, consisting of a fiber optic in the waveguide coupled to a photomultiplier tube in a Faraday cage, was installed to monitor light from the microwave window. Figure 67 shows an output trace from the arc detector overlaid with the power signal trace (shot 12251). The signal from the arc detector begins to increase just as the measured microwave power begins to drop. The PMT saturates before the microwave signal is completely cut off by the plasma from the window flashover.

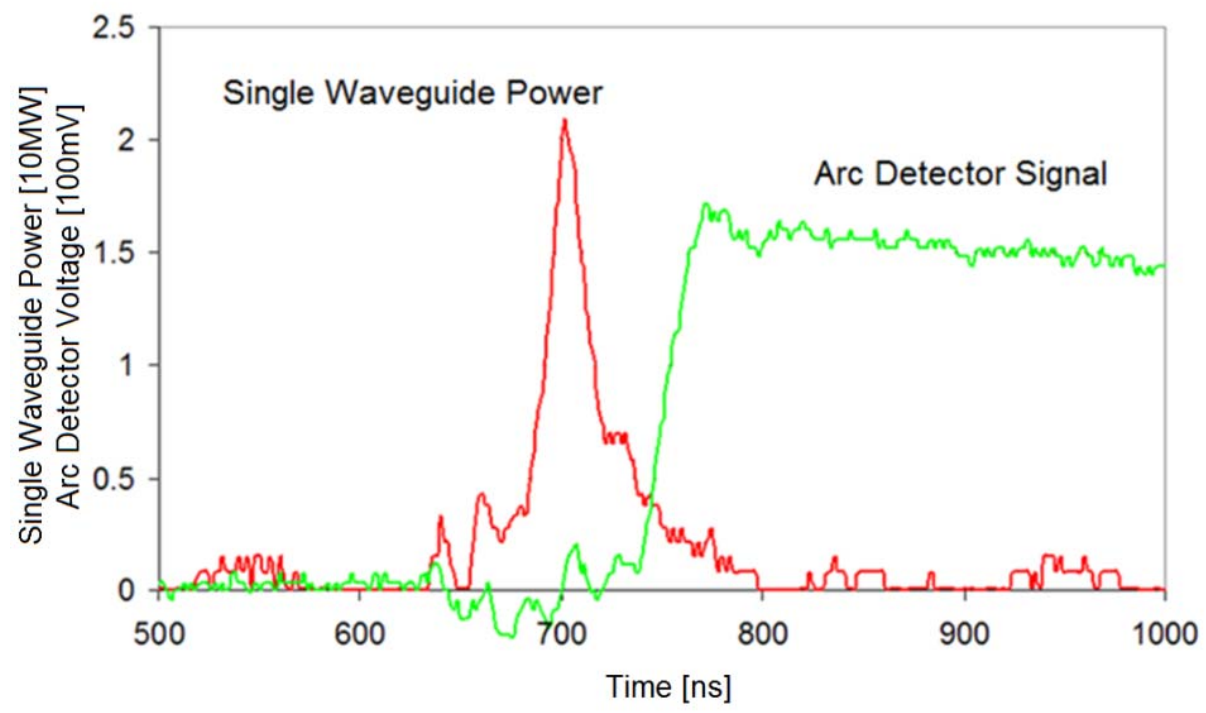

Figure 67. Single waveguide microwave power and arc detector output for magnetron in original window configuration (shot 12251).

After approximately 400 shots, the microwave loads were removed from the ends of each of the extraction waveguides and the damaged microwave windows were photographed. One of these photographs is displayed in Figure 68. The microwave window photograph clearly shows the tracking, caused by repeated window flashover discharges, centered in the region of highest RF 
electric field for the $\mathrm{TE}_{10}$ waveguide mode. After further analysis of the windows, it was verified that all damage to the window had occurred on the side exposed to vacuum. The $\mathrm{SF}_{6}$ side of the window was found to be pristine.

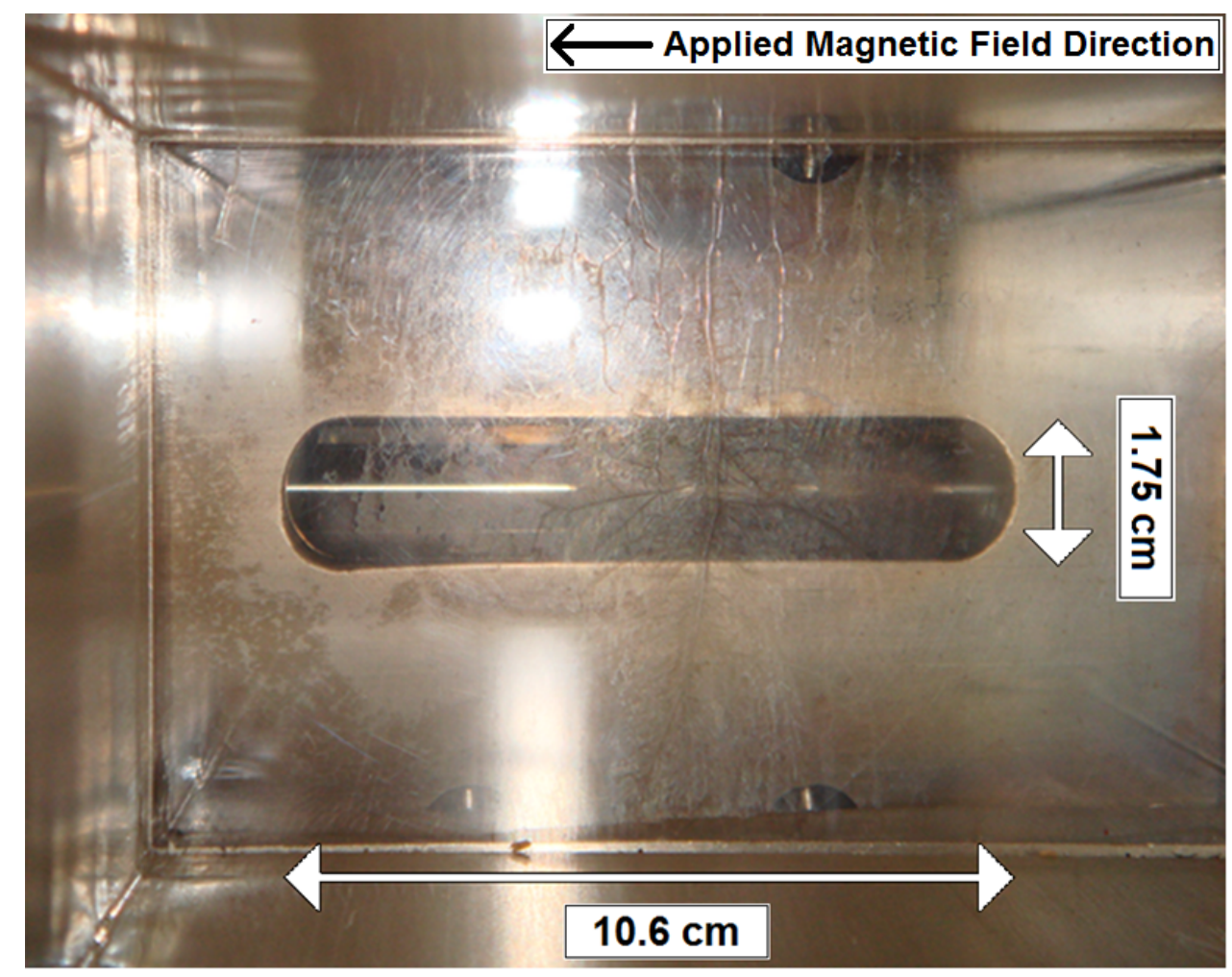

Figure 68. Photograph of the damaged microwave window from the original configuration, looking toward the coupling slot and the center of the magnetron.

Once the AFRL vacuum side power couplers and new window mounts were installed, all indications of window flashover ceased. Figure 69 (a) and (b) depict microwave power data and time frequency analysis for magnetron shot 12294. In this shot, the magnetron windows were in the new, upgraded configuration and the magnetron was oscillating in the m-mode. 


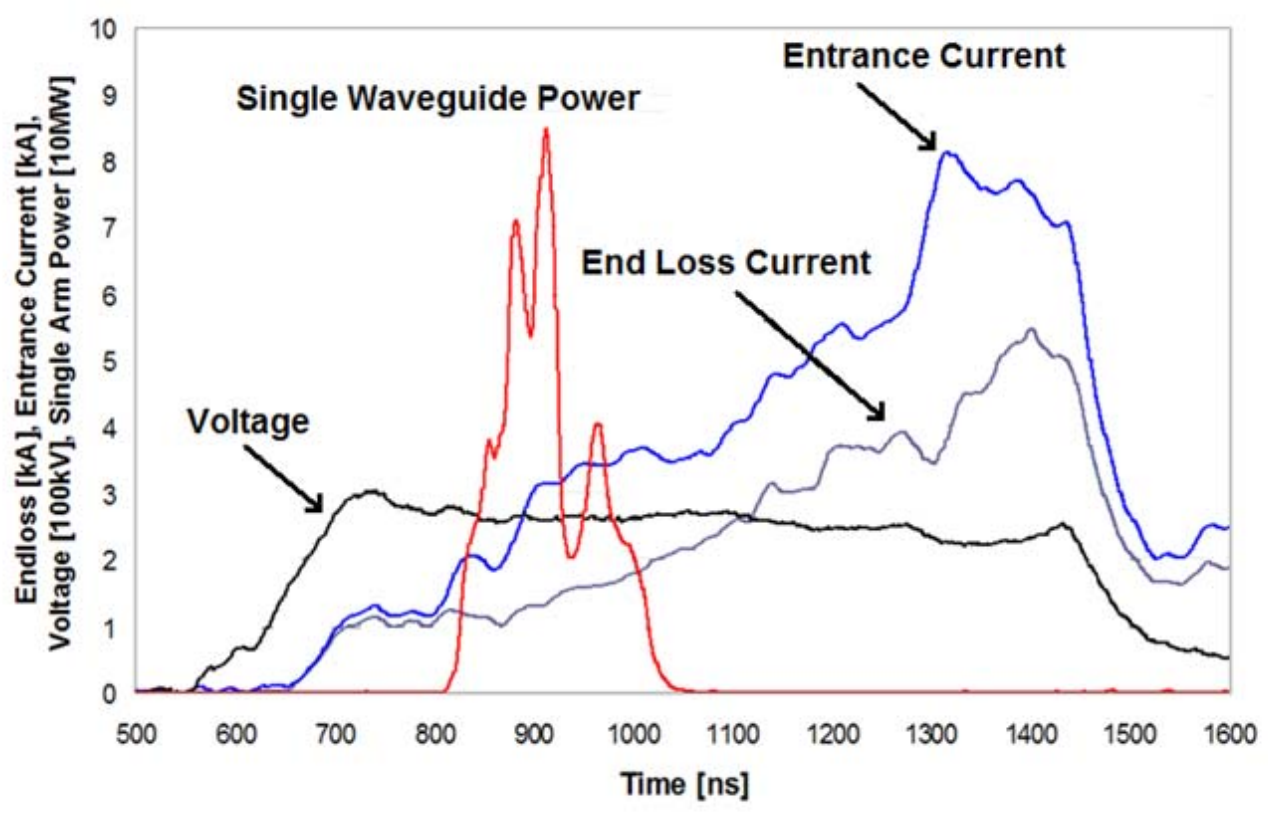

(a)
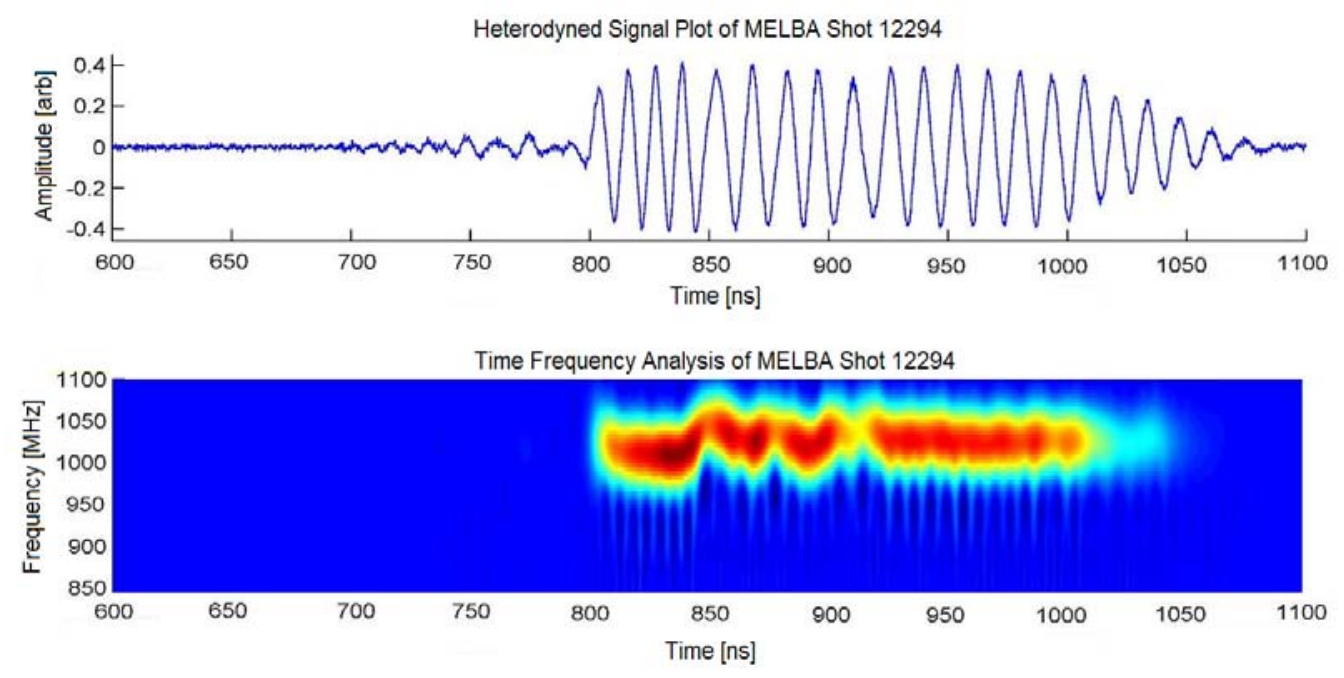

(b)

Figure 69. Relativistic magnetron performance in the new, upgraded configuration (magnetron shot 12294). (a) Accelerator voltage, entrance current, end loss current, and single waveguide microwave power, (b) Heterodyned signal and time frequency analysis of the microwave signal for magnetron.

Figure 70 displays a plot of single waveguide microwave power and the corresponding arc detector signal. Unlike the arc detector trace from Figure 67, 
there is no sharp jump in the detector voltage during the microwave pulse, giving further evidence that microwave window flashover is not occurring in the new configuration. The gradually increasing low level signal from the arc detector is likely due to light generated by plasma on the cathode [85].

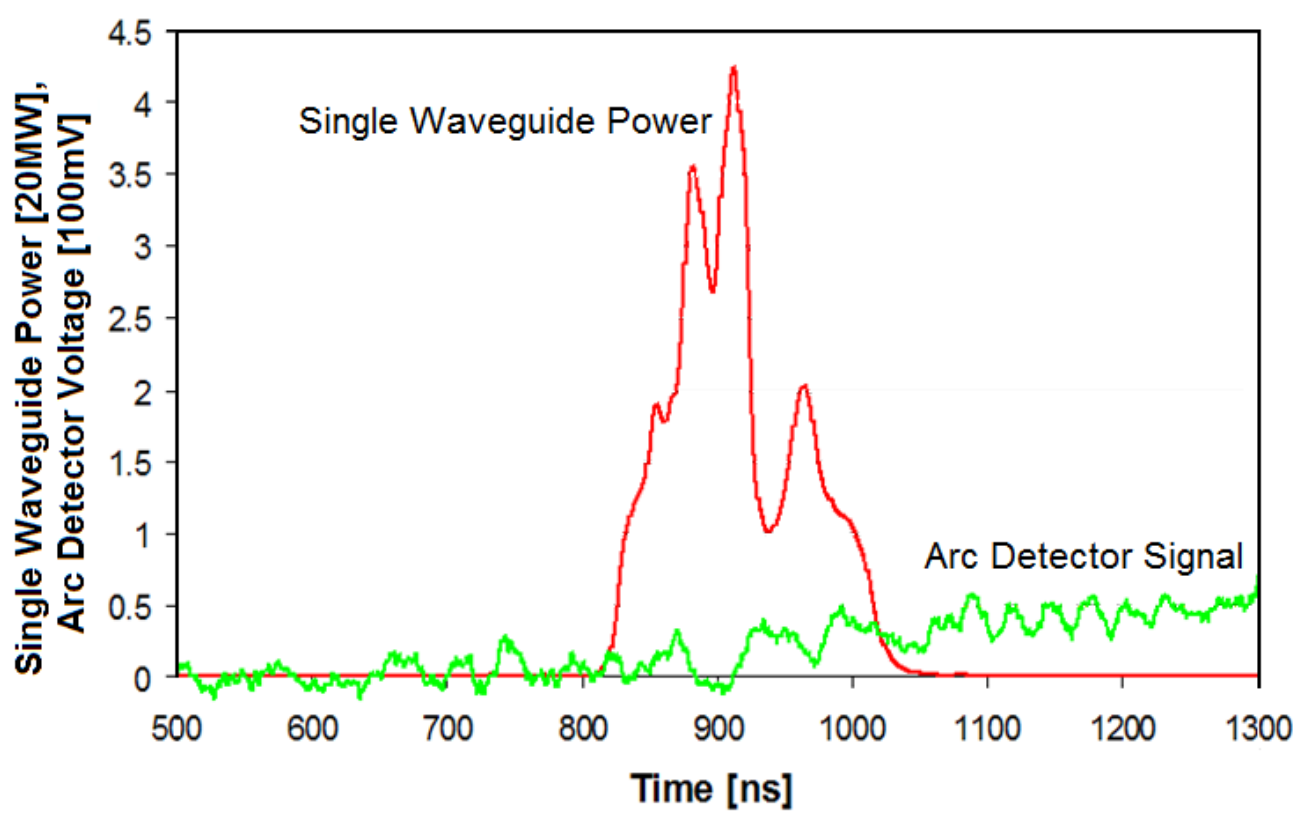

Figure 70. Single waveguide microwave power and arc detector output for magnetron in the new configuration (shot 12294).

The data displayed in Figure 71 represents magnetron shot averages for microwave pulse width and single waveguide microwave power for the original versus the upgraded window configurations. The confidence bars extend to $+/-1$ standard deviation from the mean. It is evident that there exists a dramatic increase in the measured peak output powers and pulse widths of the microwave pulses from the magnetron after the microwave windows are installed in the new, upgraded configuration. The upgraded microwave windows were inspected after 
approximately 900 magnetron shots (which included both the window evaluation shots and the second and third magnetic priming experimental series shots) and were found to exhibit no indications of damage.

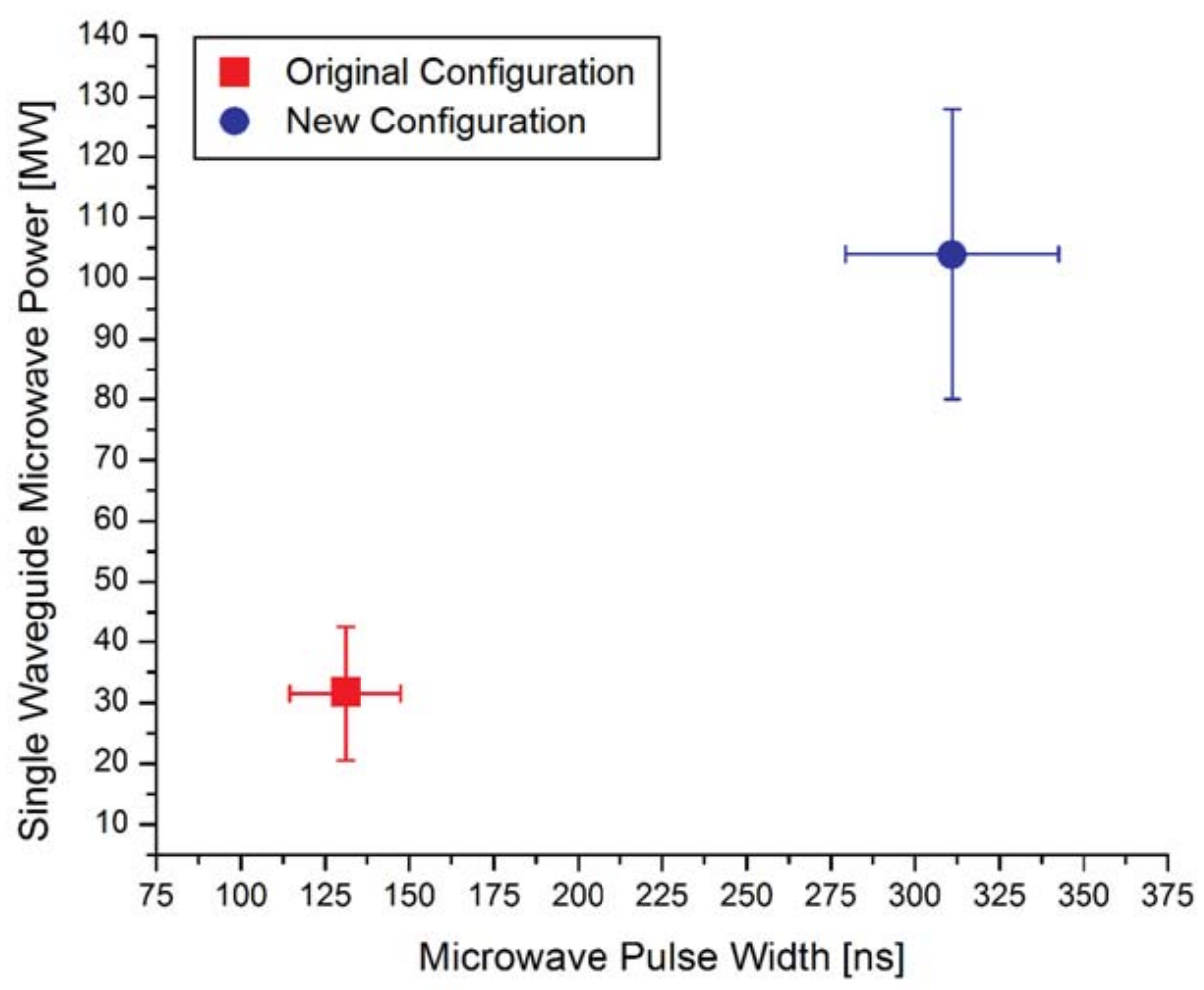

Figure 71. Microwave power and pulse length for original versus new upgraded window configurations.

It is important to note that while the measured power and pulse width varied greatly between the two configurations due to the difference in microwave window performance, it is expected that the actual range of power and duration of the microwave pulses emitted interior to the magnetron in the two cases was approximately the same. This expectation is based on the fact that the 
magnetron operating voltage and range of magnetic fields used in the two configurations were the same.

The dramatic change in microwave window performance between the two configurations prompted an investigation into the source of the window flashover in the poorly performing, original case. Since the primary difference between the original and new window configurations was the location of the window in the output waveguides, proximity to the microwave coupling slots (apertures) appeared to be a likely contributing factor. Results from previous simulations of the UM/L-3 relativistic magnetron in a variety of operating conditions show that it is extremely unlikely that electrons originating from the cathode are responsible for impacting the microwave windows $[63,64,66,67,86]$.

The other likely sources of electrons found in close proximity to the microwave windows are the microwave apertures. In the construction of the anode assembly of the UM/L-3 relativistic magnetron, edges of the apertures (visible in Figure 68) were cut at approximately a 90 degree angle to the outer surface. If exposed to a high amplitude RF electric field, these aperture edges could act as knife-edge field emitters [87-89], releasing electrons that could strike the window surface and initiate flashover [90]. Closer proximity to both the aperture and the magnetron itself would also increase the intensity of UV radiation and soft $\mathrm{X}$-rays interacting with the window surface, which has also been shown to increase the likelihood of flashover on dielectric surfaces in both vacuum [77] and atmospheric pressure conditions [78]. 


\subsection{Simulation Parameters and Results}

In order to test the hypothesis that impacts by electrons emitted from the microwave apertures are responsible for initiating window breakdown, a model of the UM/L-3 relativistic magnetron was simulated in the AFRL-designed electromagnetic particle-in-cell (PIC) code, ICEPIC. Figure 72 (a) and (b) depict cross-sections of the simulation geometry. In this simulation, the microwave aperture indicated in Figure 72 (a) and (b) was allowed to emit electrons at electric fields greater that $7.5 \mathrm{MV} / \mathrm{m}$. This electric field value is consistent with published field emission thresholds of stainless steel [91]. Due to the mesh properties of ICEPIC, the 90 degree knife edges of the aperture are effectively rounded to a radius on the order of one half of the cell size. Because cells with side dimensions of approximately $2 \mathrm{~mm}$ were used in the aperture region, ICEPIC will under-predict the field enhancement that would be expected in the actual experiment. 


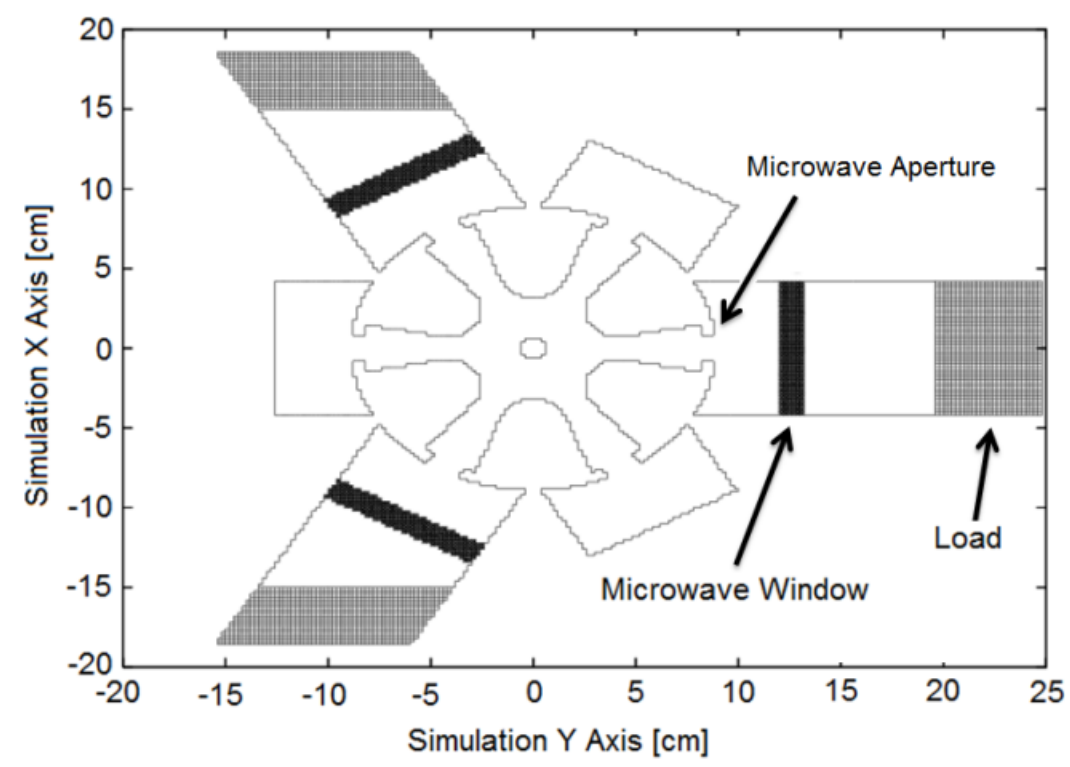

(a)

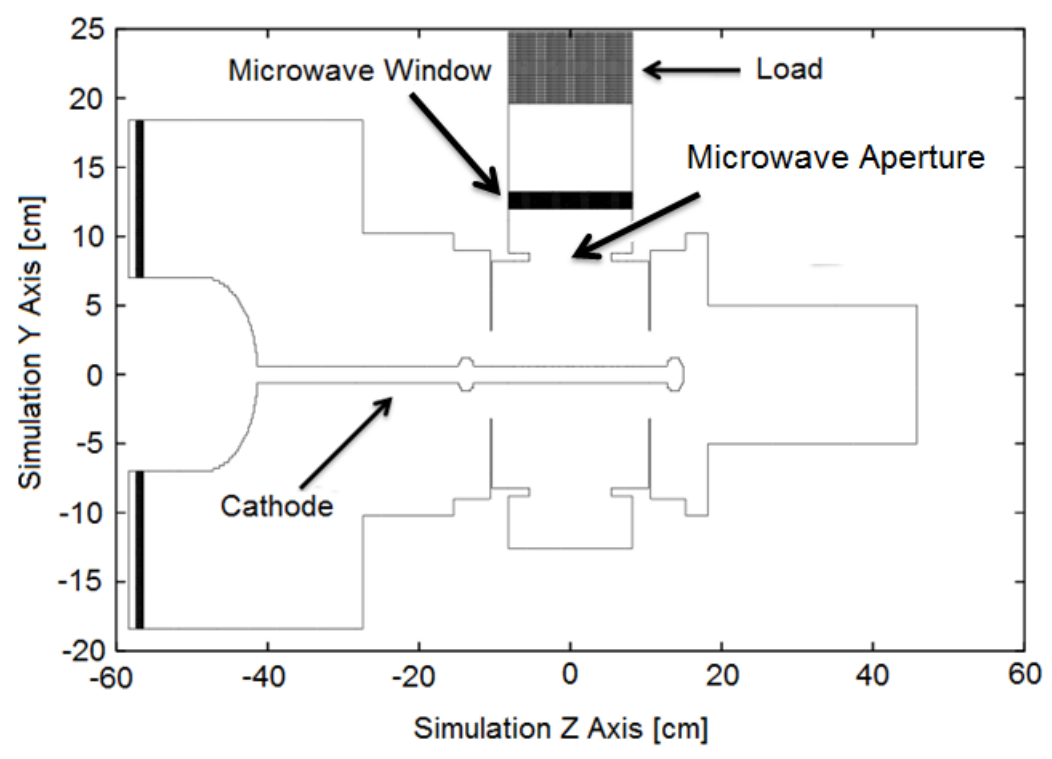

(b)

Figure 72. Simulation model geometry. (a) XY Plane Cross Section (units in cm). (b) $Y Z$ Plane Cross Section (units in $\mathrm{cm}$ ) [ICEPIC data courtesy of P. J. Mardahl].

In these simulations, field emission was enabled, but phenomenological models for secondary emission or plasma desorption were not used. Because of 
this, electrons impacting the microwave windows are accumulated as stored negative charge on the window face. The microwave windows were assigned a relative permittivity of 2.6, consistent with published values for PMMA at $1 \mathrm{GHz}$ [92].

Magnetic field, microwave power, and microwave frequency are the three parameters expected to be most important to the physical mechanism initiating the window flashover events. The magnet coil geometry used in the experiment was replicated in the simulation to provide as realistic of a magnetic field profile as possible. The simulated magnet coil currents were adjusted such that the magnetic field at the center of the magnetron was the median value for the experimental operating range, in this case $0.27 \mathrm{~T}$, oriented along the $+Z$ axis. The magnetron input voltage characteristics were selected to achieve microwave outputs comparable to those observed in the experiment. The plot displayed in Figure 73 gives output traces for single waveguide output power, input voltage, anode current, and magnetron impedance as a function of time for the case in which aperture emission was enabled. At steady state, the simulation magnetron oscillated in the 2pi/3-mode, emitting approximately $115 \mathrm{MW}$ per waveguide at a frequency of $952 \mathrm{MHz}$. 


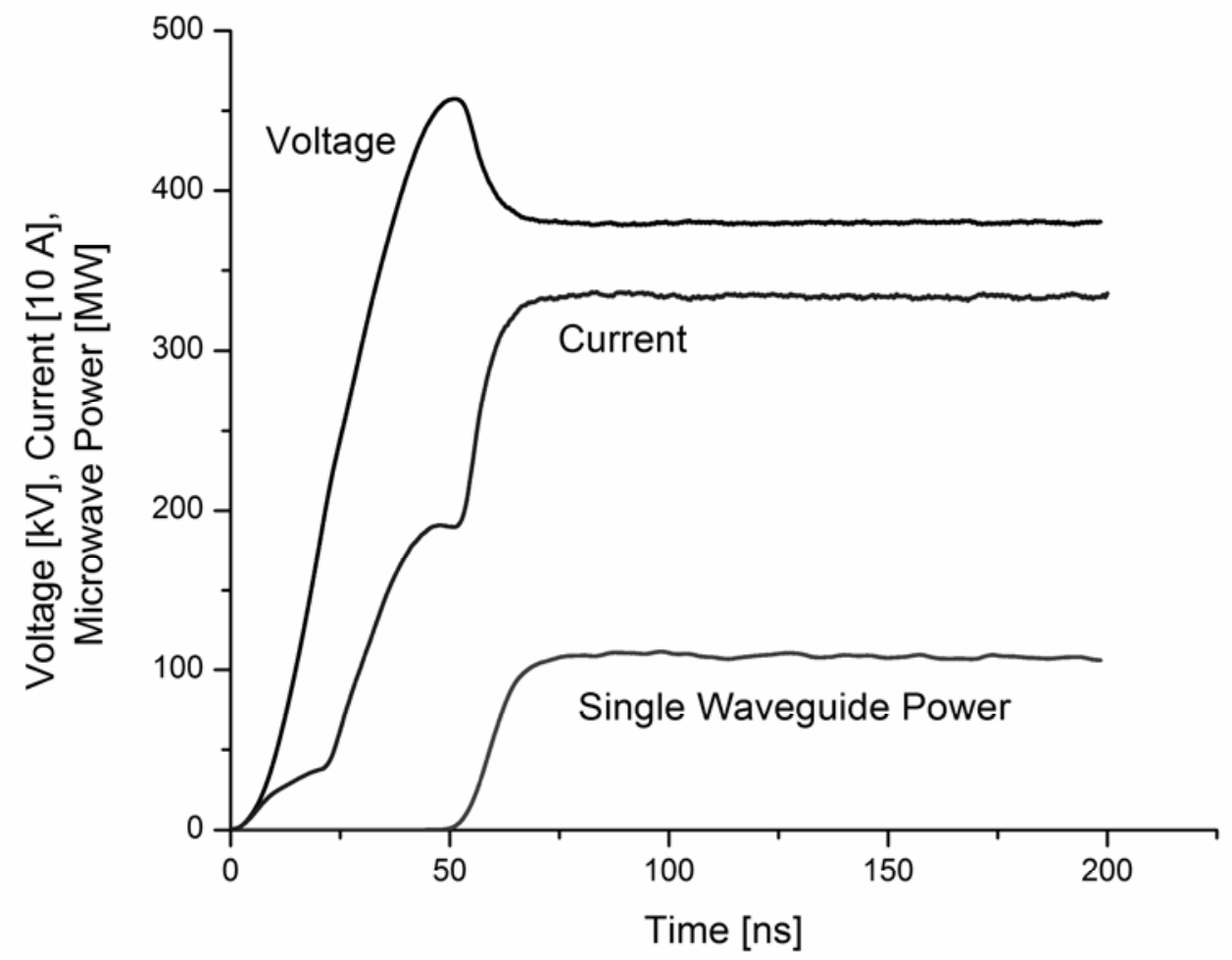

Figure 73. Input voltage, anode current, and average single waveguide microwave power for the magnetron simulation (aperture emission enabled) [ICEPIC data courtesy of P. J. Mardahl].

Once the RF power is such that the $7.5 \mathrm{MV} / \mathrm{m}$ field emission threshold of the aperture is exceeded, the emitted electrons form into an oscillating cloud, part of which remains between the aperture and the window and part of which moves back and forth across the aperture from the coupling cavity to the magnetron anode cavity. Figure $74(a)$ and (b) depict cross sections of the operating magnetron 199.343 ns into the simulation. 


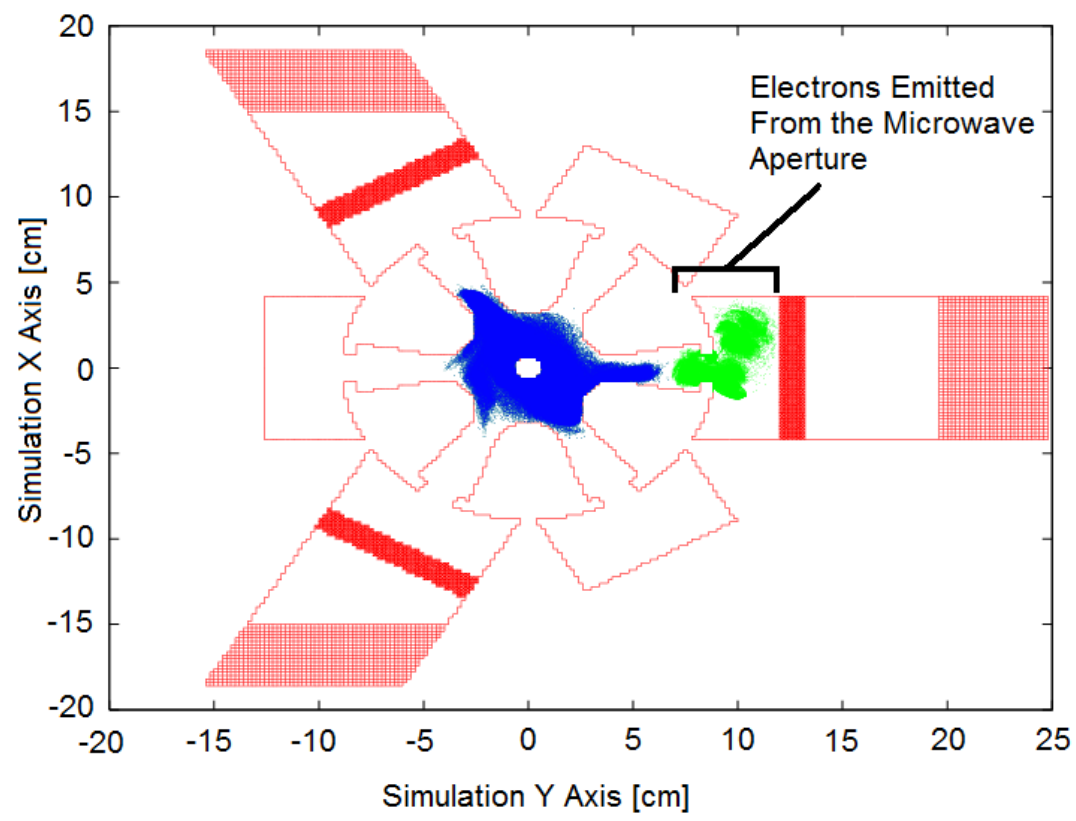

(a)

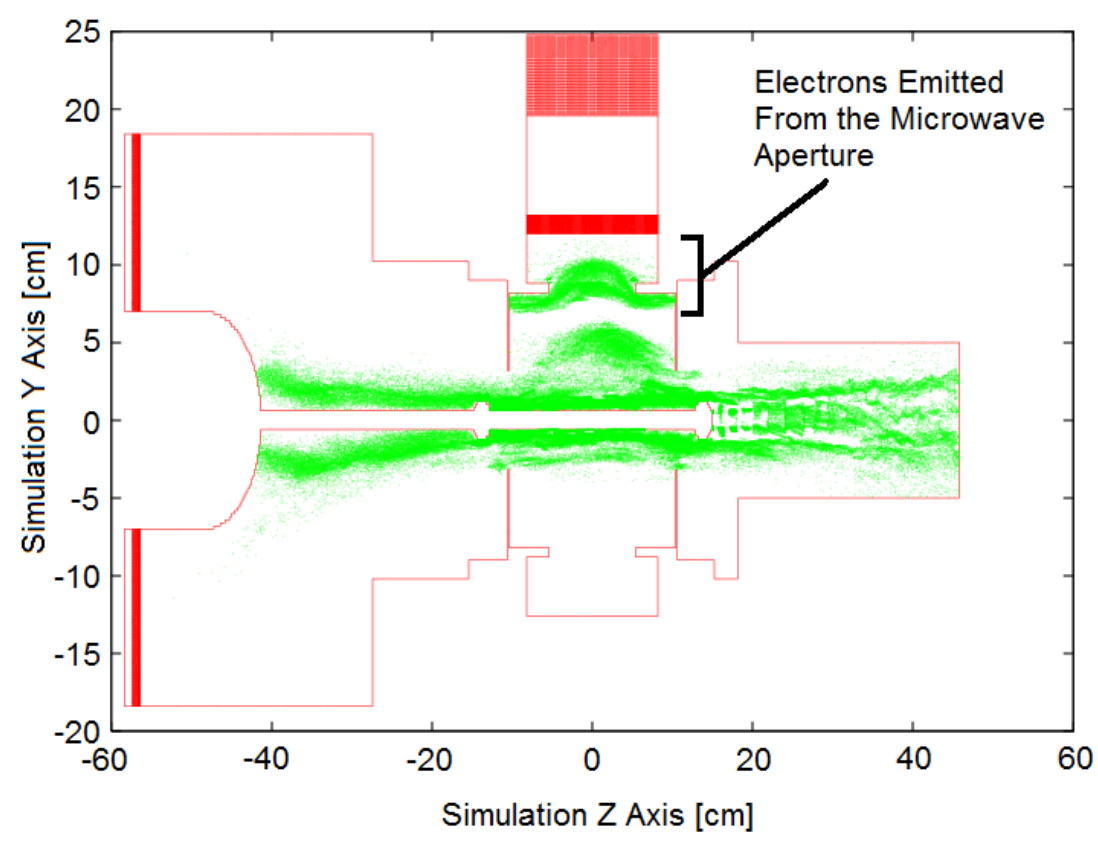

(a)

Figure 74. Cross sections of the operating simulation magnetron at $t=199.343 \mathrm{~ns}$, showing the location of the electrons emitted from the microwave aperture. (a) X-Y plane cross section (cathode electrons are given a darker color for contrast). (b) $Y-Z$ plane cross section [ICEPIC data courtesy of P. J. Mardahl]. 
The portion of the aperture electron cloud that remains between the aperture and the microwave window forms into a rotating column with a rotational axis oriented along the simulation Z-axis. The centroid of the cylindrical cloud, when viewed in the $x-y$ plane, remains in the same position throughout the full RF cycle. Figure 75 (a-d) illustrate this behavior. Reversing the polarity of the magnet coils, such that the magnetic field is oriented in the $-Z$ direction, causes the symmetry of the aperture electron cloud to flip across the $X=0 \mathrm{~cm}$ plane in the simulation.

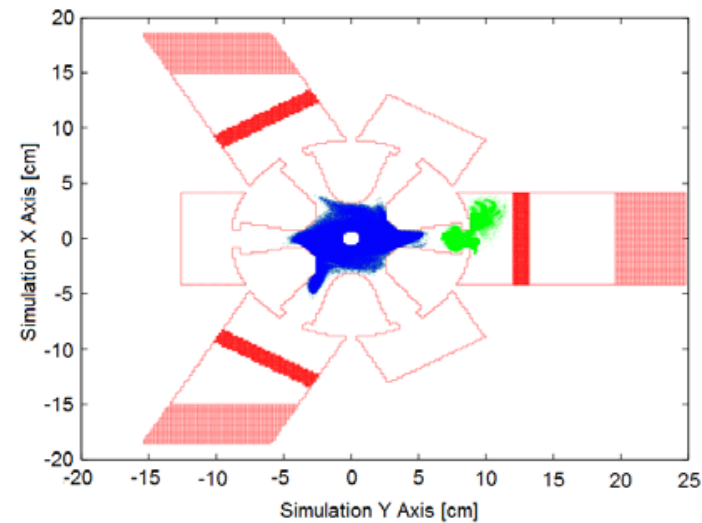

(a)

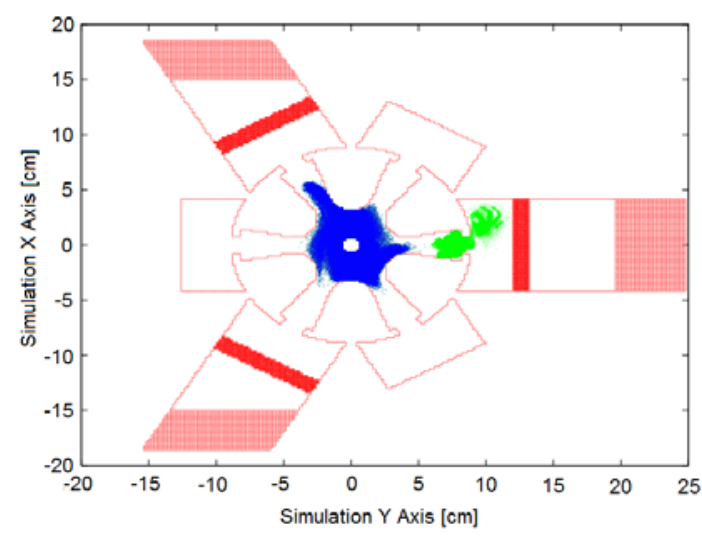

(c)

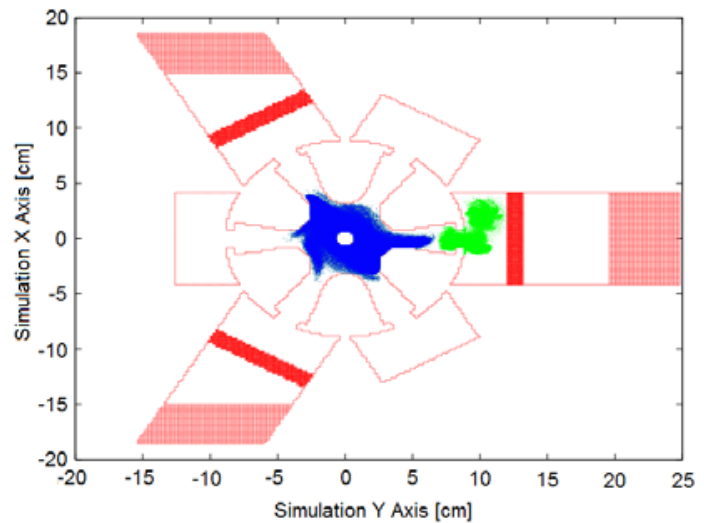

(b)

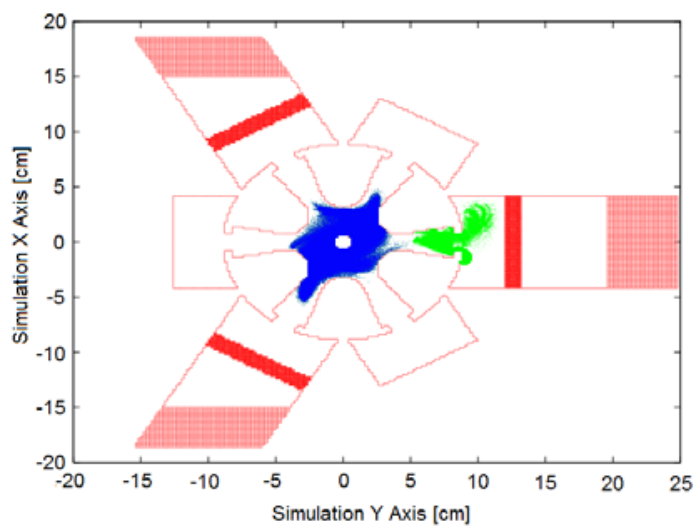

(d)

Figure 75. XY plane cross section of the simulation, showing the cathode electrons (dark blue) and the aperture electrons (light green) over one RF cycle: (a) 198.999 ns, (b) 199.293 ns, (c) 199.539 ns, and (d) 199.882 ns [ICEPIC data courtesy of P. J. Mardahl]. 
In the simulation, the first electron macroparticle impact on the microwave window occurred at $64.472 \mathrm{~ns}$, when the single waveguide microwave power reached $90 \mathrm{MW}$. Following the initial particle impact, charge steadily accumulated on the window surface at a rate of $75.8 \mathrm{~mA}$, as shown in Figure 76. ICEPIC self-consistently models the charging of the window due to the continued electron impacts and the effect of this window charging on further electron collisions. As the window charge builds, it is expected that more of the impinging electrons are repelled, thus causing the measured electron impact rate in the simulation to be lower than would be observed if a window neutralization model (such as secondary emission, plasma formation, or a gradual discharge due to nonzero window conductance) was included. 


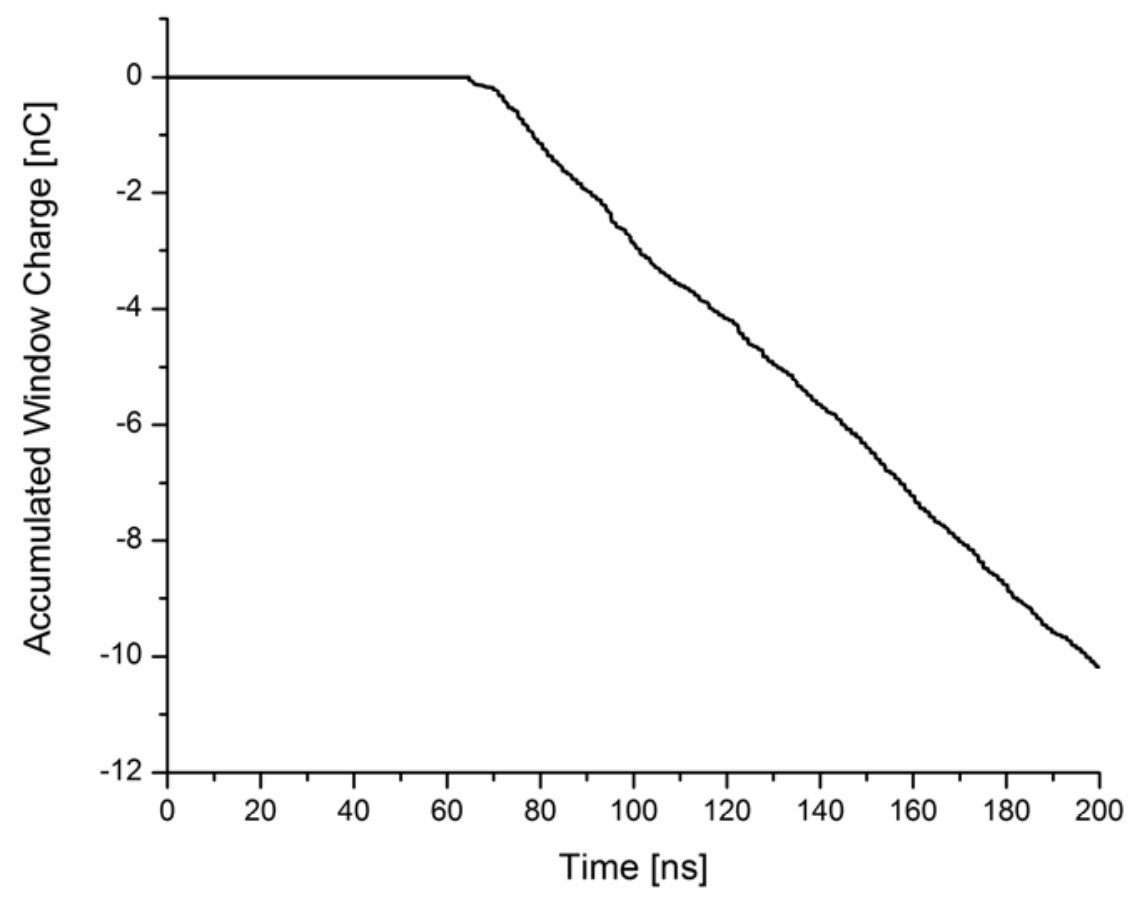

Figure 76. Accumulated charge integrated over the surface of the microwave window [ICEPIC data courtesy of P. J. Mardahl].

Figure 77 depicts a plot of the particle impact locations on the surface of the microwave window. The window boundaries and a projection of the microwave aperture in the $\mathrm{X}-\mathrm{Z}$ plane are overlaid on the plot, for reference. Particle impact locations on the microwave window correlate to the same $X-Z$ coordinate region as occupied by the rotating column of aperture electrons shown in Figure 75 (a-d). 


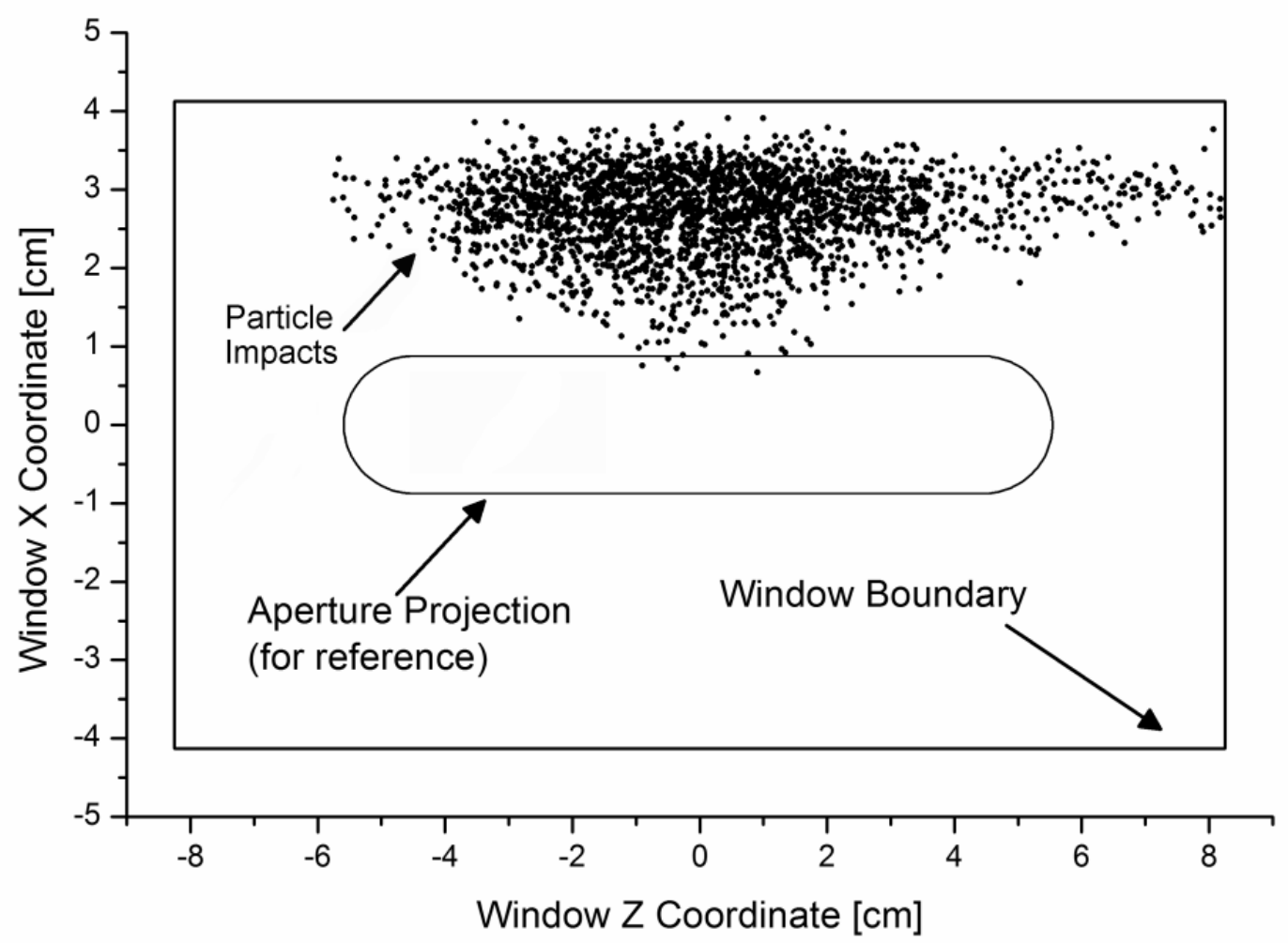

Figure 77. Particle impact locations on the front face of the microwave window [ICEPIC data courtesy of $P$. J. Mardahl].

The kinetic energy and impact angle distributions for all particle impacts are displayed in Figure 78 (a) and (b). Mean kinetic energy for the electron impacts was found to be $33 \mathrm{keV}$ with a standard deviation of $14 \mathrm{keV}$. The electrons were found to have impact angles with a mean of 54 degrees from normal incidence with a standard deviation of 17 degrees. 


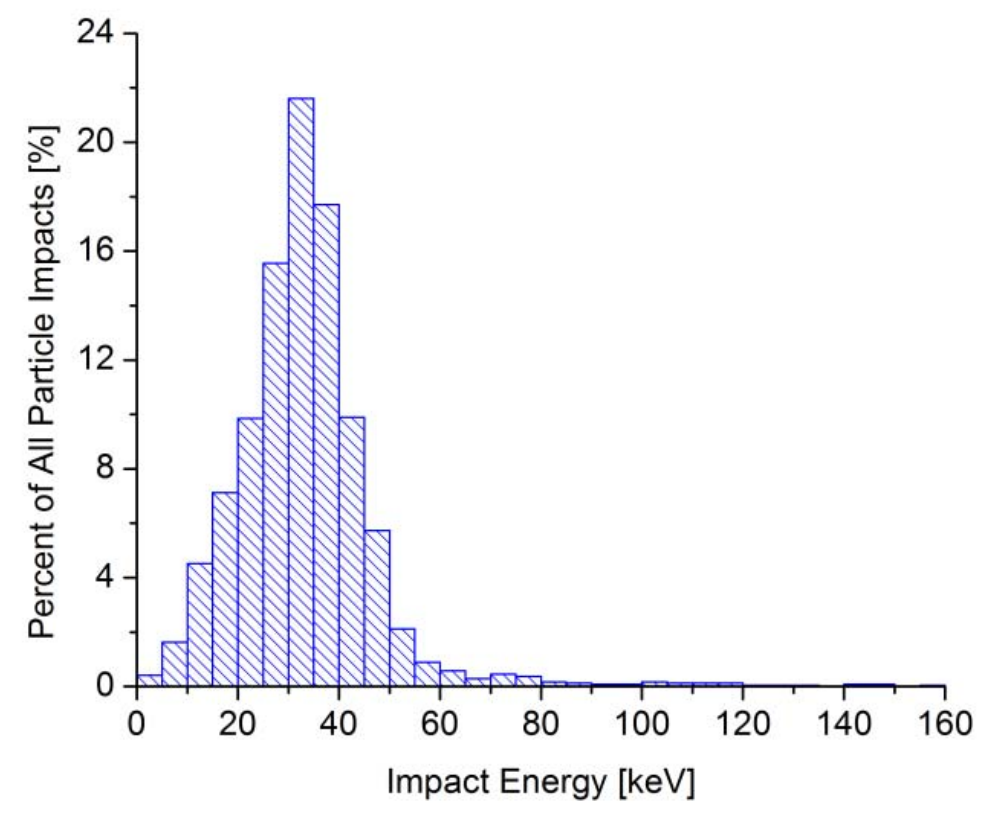

(a)

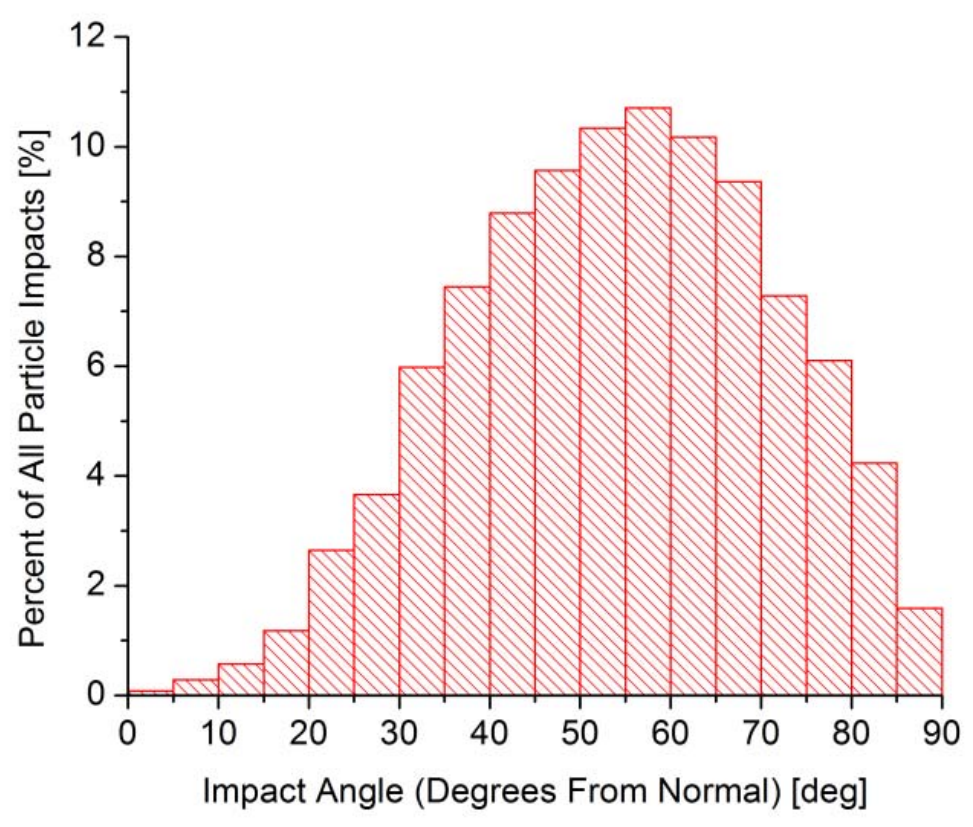

(b)

Figure 78. Distribution functions for (a) impact energy and (b) impact angle for all particle impacts on the microwave window [ICEPIC data courtesy of P. J. Mardahl]. 
Additional simulations were performed with the microwave windows removed but with all other parameters the same. In this case, all magnetron performance metrics, including microwave power, were effectively the same as the case with the windows included. In the no-window case, the electron cloud was found to have a maximum radial extent of $3.5 \mathrm{~cm}$ beyond the microwave aperture.

\subsection{Analysis and Discussion}

Although the emission of secondary electrons was not modeled in the simulation, the impact energy and impact angle of each electron strike, displayed in Figure 79 , can be used to make predictions about the probability of the emission of secondary electrons. 


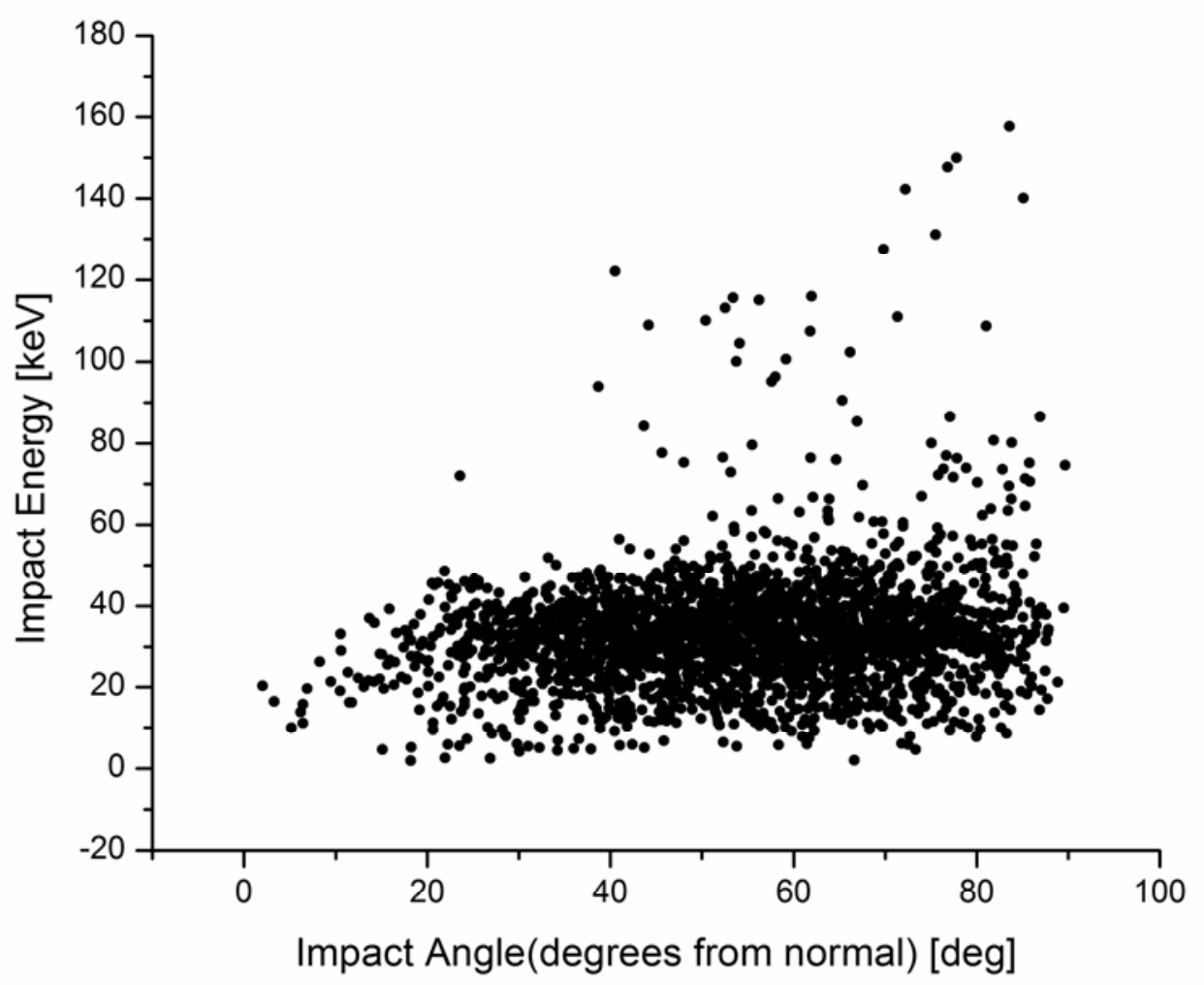

Figure 79. Particle impact energy plotted as a function of impact angle for all particles striking the microwave window [ICEPIC data courtesy of P. J. Mardahl].

In order to calculate the predicted secondary electron yield of each electron impact, we use Vaughan's empirical formulas $[93,94]$. According to Vaughan, the angle-dependant secondary emission coefficient for a given electron impact event, $\delta(\theta)$ can be calculated using the equation

$$
\delta(\theta)=1.125 * \delta_{\max }(\theta) * v^{-0.35}
$$

as long as the parameter $\mathrm{v}$, calculated as

$$
v=\frac{E_{i}-E_{0}}{E_{\max }(\theta)-E_{0}}
$$


is equal to 3.6 or greater. Impacts in the simulation had v values ranging from 4.5 to $318.4 . E_{i}$ is the energy with which a given electron impacts the surface of the microwave window. $\delta_{\max }(\theta)$ and $E_{\max }(\theta)$, the maximum secondary electron yield for a given angle and the impact energy at which maximum yield occurs, respectively, are found using

$$
E_{\text {max }}(\theta)=E_{\text {max }}(0)\left(1+\frac{k_{S E} \theta^{2}}{2 \pi}\right)
$$

and

$$
\delta_{\max }(\theta)=\delta_{\max }(0)\left(1+\frac{k_{s \delta} \theta^{2}}{2 \pi}\right) .
$$

$\delta_{\max }(0)$ is defined as the maximum secondary electron yield at normal incidence, and $E_{\max }(0)$ is the impact energy at which this maximum yield occurs. The constants $\mathrm{k}_{\mathrm{SE}}$ and $\mathrm{k}_{\mathrm{s} \delta}$ are surface "smoothness factors" which, for this analysis, are assigned a default value of $1 . E_{0}$ is the minimum impact energy at which any secondary electrons at all are generated. The specific values of $\delta_{\max }(0), E_{\max }(0)$ for PMMA used in this analysis $(2.2,370 \mathrm{eV}$, respectively) were experimentally determined by Boubaya, et. al. [95]. $E_{0}$ is approximated to have a value of $0 \mathrm{eV}$.

Figure 80 displays a histogram plot of the calculated secondary emission coefficients for each of the window impacts. The mean secondary yield for all impacts was found to be 0.65 with a standard deviation of 0.12 . Thus, on average, only $65 \%$ of the electrons that originate at the microwave aperture and 
strike the microwave window are predicted to yield a secondary electron (with the primary electron considered to be lost) due to their high impact energies.

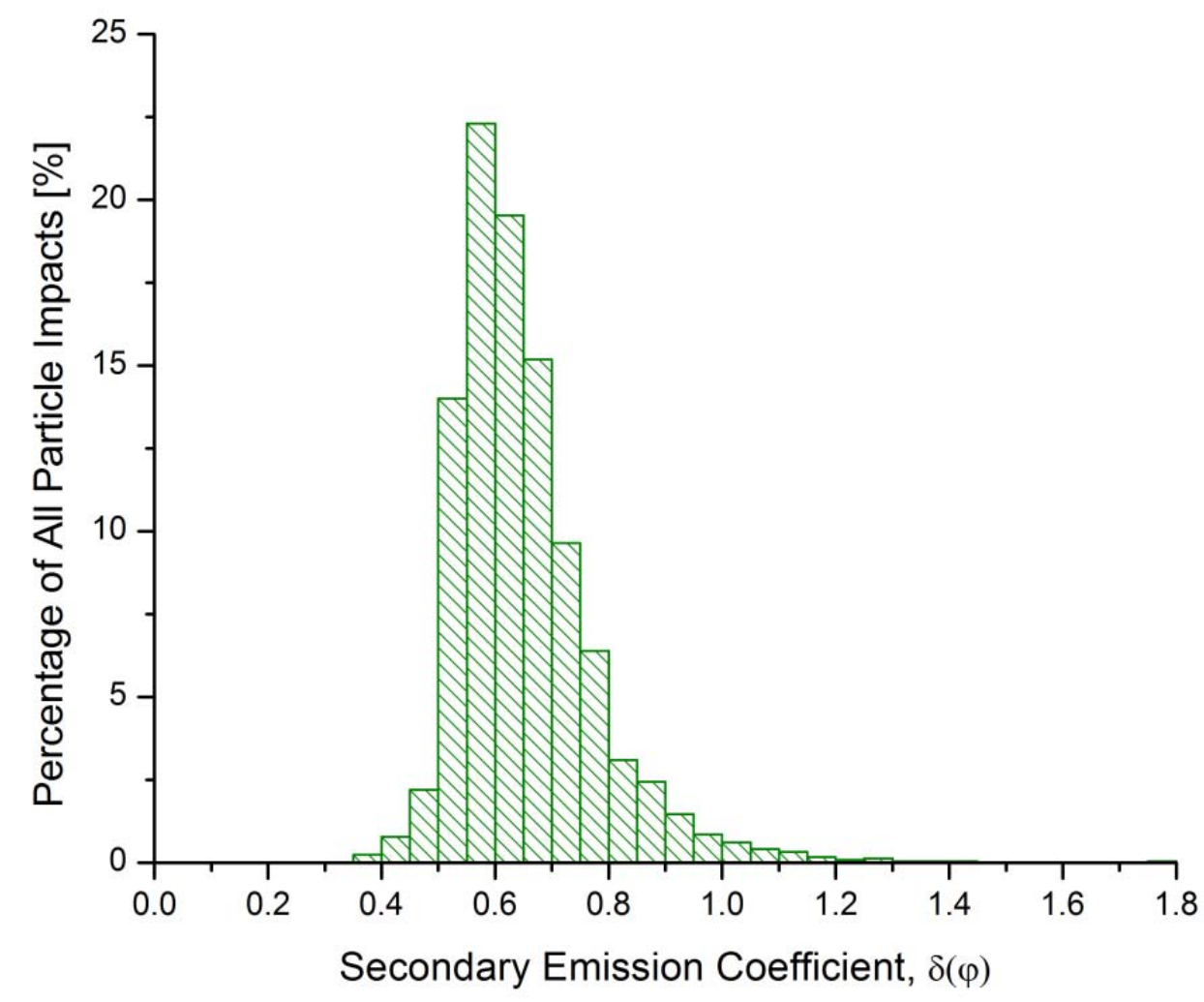

Figure 80. Distribution of predicted secondary emission yields for all simulation particle impacts [ICEPIC data courtesy of P. J. Mardahl].

Although the initial population of secondary electrons is predicted to be smaller than that of the impacting primary electrons from the aperture, the secondary particles are typically emitted with energies of the order of the work function of the window material $[96,97]$. This means that the secondary electrons will likely be on the order of $5 \mathrm{eV}$ [98]. These lower energy electrons are then susceptible to initiating discharges through multiplication mechanisms such as single surface dielectric multipactor $[68,69,99]$. Additionally, window impacts 
from both primary and secondary electrons on the window surface are likely to result in gas and ion desorption from the window [100,101], further adding to the likelihood of window flashover plasma.

\subsection{Implications on Magnetic Priming Results}

It is suspected that microwave window breakdown was occurring during the first magnetic priming experimental campaign $(4 \mathrm{~cm}$ wires, unbalanced loading), as the upgrades added to the experiment to prevent this phenomenon did not occur until just prior to the second experimental campaign. Peak power data from this first campaign showed that the magnetically primed case had higher peak powers than the unprimed case, which was in contrast to the rest of the simulation data and second and third experimental campaigns. The data from the first campaign also indicated a faster growth rate for the m-mode shots. If one assumes that each window flashover event begins at a specific power threshold (not an unreasonable assumption, based on the window breakdown simulation data) and progresses to microwave cutoff over a relatively constant timescale, the faster m-mode growth rate would appear to give higher overall peak powers. Because the single waveguide powers observed in the first experiment are close to the original configuration shot series, displayed in Figure 71 , which were confirmed to be flashing over, this seems to be a likely cause for the discrepancy in expected and measured power output. Thus, in this regard, the "peak power" metric for the first magnetic priming experimental campaign would be more correctly named "peak power at cutoff". 
Experimental data gathered during the second and third magnetic priming experimental campaigns suggests that the root cause of the window breakdown events, the electron (and plasma) plume from the microwave apertures, may still be affecting magnetron performance to a limited degree. Figure 81 displays a plot of the individual waveguide microwave output signals for MELBA-C shot 12435. Although each of the microwave traces start and end at the same time, the individual trace shapes are markedly different. Even if the microwave windows are not flashing over, plasma formation in the region of the aperture could reflect a portion of the microwave power back into the magnetron, which would result in behavior such as that depicted in Figure 81. This effect did not occur on every shot and the magnitude of the effect appeared to vary randomly across the three waveguides for each of the three magnetic priming experimental campaigns.

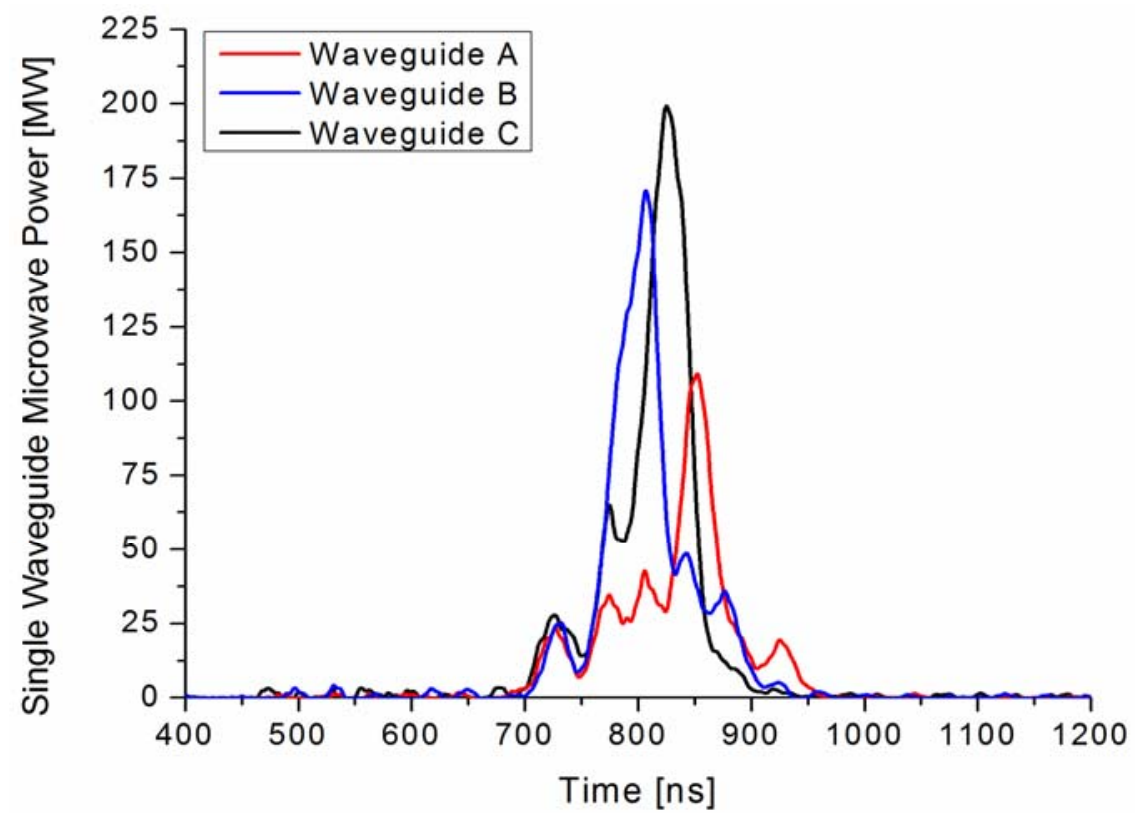

Figure 81. Individual waveguide power traces for MELBA-C shot 12435 showing variations in measured power among the waveguides. 


\section{Chapter 7}

\section{Conclusions}

The magnetic priming concept implemented in the UM/L-3 relativistic magnetron involves placing magnetic structures within the magnetron itself. The embodiment of this concept was realized by embedding sets of three high permeability wires, spaced 120 degrees apart, in the cathode and anode of the 6vane $U M / L-3$ relativistic magnetron. These priming geometries were designed to enhance the operation of the $\pi$-mode ( $\mathrm{N} / 2$ symmetry for an $\mathrm{N}$-vane magnetron).

Magnetostatics simulations performed indicated that in addition to the perturbations in the axially directed magnetic confinement field, there are also very strong radial and $\theta$ directed magnetic field perturbations introduced toward the ends of the wires. The magnetostatic field data was imported into the MAGIC PIC particle-in-cell simulation code and run for the case of a 6-vane relativistic magnetron. The particle-in-cell simulations yielded the best magnetically primed results when shorter wire lengths (on the order of $4 \mathrm{~cm}$ long) were used. Longer wires were found to cause unstable operation of the simulated magnetron. The magnetically primed simulation magnetron was found to start oscillating in the $\pi-$ mode significantly faster than the unprimed simulation magnetron. The 
magnetically primed cases suffered from reduced steady state power, compared to the unprimed case.

Three magnetic priming experiments were performed on the UM/L-3 relativistic magnetron. The first experiment involved magnetically priming the $\pi-$ mode of the relativistic magnetron using a set of three, $4 \mathrm{~cm}$ long nickel-iron alloy (Hymu 80/Permalloy) wires with diameters of 0.254 centimeters, spaced 120 degrees apart and embedded in the cathode. In this experiment, magnetic priming was demonstrated to increase the percentage of $\pi$-mode shots by $15 \%$ over the baseline case. Mean peak power for m-mode shots was found to be higher in the magnetically primed case when compared to the unprimed case (66 ns primed versus 50 ns unprimed). Magnetic priming was also shown to reduce the start-oscillation time of the $\pi$-mode. Starting time in the magnetically primed case was $64 \%$ less than the unprimed case. Magnetron starting current for the magnetically primed $\pi$-mode exhibited a reduction to $69 \%$ of the unprimed baseline starting current.

The second magnetic priming experiment also utilized sets of three, highpermeability Permalloy wires spaced 120 degrees apart and embedded in the cathode. The wire sets used in this experiment had lengths of $4 \mathrm{~cm}, 8 \mathrm{~cm}, 12$ $\mathrm{cm}, 16 \mathrm{~cm}, 20 \mathrm{~cm}$ and $27 \mathrm{~cm}$. Individual wire diameters were equal to $0.127 \mathrm{~cm}$. In this experiment, the $4 \mathrm{~cm}$ and $8 \mathrm{~cm}$ wire cases exhibited the greatest degree of mode control, with $73 \%$ and $76 \%$ of the magnetron shots occurring purely in the $\pi$-mode, compared with only $45 \% \pi$-mode shots in the baseline case. In addition, the $4 \mathrm{~cm}$ and $8 \mathrm{~cm}$ wire cases almost completely suppressed purely 
$2 \pi / 3$ shots. The percentage of bimodal shots starting in the $2 \pi / 3$-mode then jumping to the $\pi$-mode in the $4 \mathrm{~cm}$ and $8 \mathrm{~cm}$ wire cases was approximately the same as in the unprimed case. Little change in percentage of $\pi$-mode shots was observed in the $12 \mathrm{~cm}$ wire and $16 \mathrm{~cm}$ wire cases. In the two longest primingwire cases, $20 \mathrm{~cm}$ wires and $27 \mathrm{~cm}$ wires, no $\pi$-mode shots were observed. Moreover, all magnetron shots in the $27 \mathrm{~cm}$ wire case oscillated purely in the 2ா/3-mode.

While the $4 \mathrm{~cm}$ cathode wire case in the second experimental campaign showed excellent mode control, the average microwave power and pulse width values for the $\pi$-mode shots in this case were both found to be approximately $80 \%$ of the unprimed $\pi$-mode shots. The other wire length cases showed no statistical difference from the unprimed case. The magnetron was observed to start up faster in the $\pi$-mode in the $4 \mathrm{~cm}$ wire, $8 \mathrm{~cm}$ wire, and $12 \mathrm{~cm}$ wire cases, when compared to the unprimed case. Only the $4 \mathrm{~cm}$ and $8 \mathrm{~cm}$ cases reached the peak power sooner than the unprimed case. No reductions in starting current were observed between magnetically primed and unprimed cases in the second experiment. The m-mode energy efficiency for all cases, except the $4 \mathrm{~cm}$ wire case, was the same as the unprimed case. The $4 \mathrm{~cm}$ wire case exhibited a reduction in energy efficiency of $5 \%$. Compared to the other wire length cases tested during the second experiment, the $8 \mathrm{~cm}$ wires appeared to yield the best performance. The $8 \mathrm{~cm}$ wire case exhibited excellent mode control and faster start up without incurring the reductions in power and pulse length observed in the $4 \mathrm{~cm}$ wire case. 
The third magnetic priming experiment implemented the three fold magnetic perturbations using sets of $0.508 \mathrm{~cm}$ diameter high permeability nickeliron wires embedded in alternating anode vanes of the relativistic magnetron. Data sets taken for wire lengths of $10 \mathrm{~cm}, 15 \mathrm{~cm}$, and $20 \mathrm{~cm}$ were compared to the same unprimed baseline case used in the second experiment. Each of the three magnetic priming cases showed enhanced mode control over the unprimed case. The magnetically primed cases showed an average increase in the number of purely $\pi$-mode shots of around $20 \%$ over the baseline case.

In this third series, the mean peak power and mean pulse width values of the $\pi$-mode shots were both found to be lower in the $15 \mathrm{~cm}$ anode wire case than those of the baseline case (263 MW and 158 ns primed versus $416 \mathrm{MW}$ and 175 ns unprimed). The other priming cases were statistically the same as the baseline case in regard to peak power and pulse width. Reductions in $2 \pi / 3$ peak power were observed in all cases; however, only the $10 \mathrm{~cm}$ wire and $15 \mathrm{~cm}$ wire cases demonstrated reductions in $2 \pi / 3$ pulse width. All priming cases showed reductions in starting time to $57 \%$ (10 cm wire case), $85 \%$ (15 cm wire case), and $87 \%$ (20 cm wire case) of the baseline case mean starting. Only the $10 \mathrm{~cm}$ and $15 \mathrm{~cm}$ wire cases showed significant reductions in the time between voltage turn on and the time at which maximum power was reached, when compared to the unprimed case $(66 \%$ of the baseline value for the $10 \mathrm{~cm}$ wires and $89 \%$ of the baseline value for the $15 \mathrm{~cm}$ wire case). No statistically significant differences in starting current were observed in any of the cases. The $10 \mathrm{~cm}$ wire case exhibited a $5 \%$ increase in m-mode energy efficiency over the unprimed case. 
Taking into account mode control, microwave pulse characteristics, starting time, and efficiency, the $10 \mathrm{~cm}$ wire case appeared to outperform the other anode wire cases.

During the course of the magnetic priming experiments, it was determined that the dielectric microwave windows used as a vacuum barrier for the magnetron, were flashing over. The window flashover plasma was limiting microwave power and pulse width. Microwave window performance was found to improve dramatically when the windows were moved away from the magnetron anode block microwave apertures. In the original configuration, the aperture to window separation distance was $3 \mathrm{~cm}$. In this configuration, mean microwave power and pulse lengths were $35.1 \mathrm{MW}$ and $104 \mathrm{~ns}$, respectively, and window flashover occurred on nearly every magnetron shot. In the new configuration, the windows were moved outward from the magnetron such that the aperture to window separation was $33 \mathrm{~cm}$. Due to improved window performance, both microwave power and pulse length were increased by a factor of three (131 MW and $311 \mathrm{~ns})$. No window flashover was observed on any of the magnetron shots in the new configuration.

Simulations were performed using the U.S. Air Force developed particlein-cell code, ICEPIC, to investigate the cause of the change in window performance. The dramatic difference in the two cases was found to be due to electrons being emitted from the edges of the microwave apertures and striking the dielectric microwave windows. The electron cloud emitted from the aperture was found to be a relatively short range phenomenon, extending to a maximum 
radius of $3.5 \mathrm{~cm}$ beyond the aperture. This result suggests that although in the experiment the windows were moved $33 \mathrm{~cm}$ away from the aperture boundary, moving the windows only $1-2 \mathrm{~cm}$ beyond their original position might actually have been enough to avoid being struck by the aperture electrons, thus avoiding the problems with window flashover. 
Appendices 


\section{Appendix A}

\section{Calibration Data}

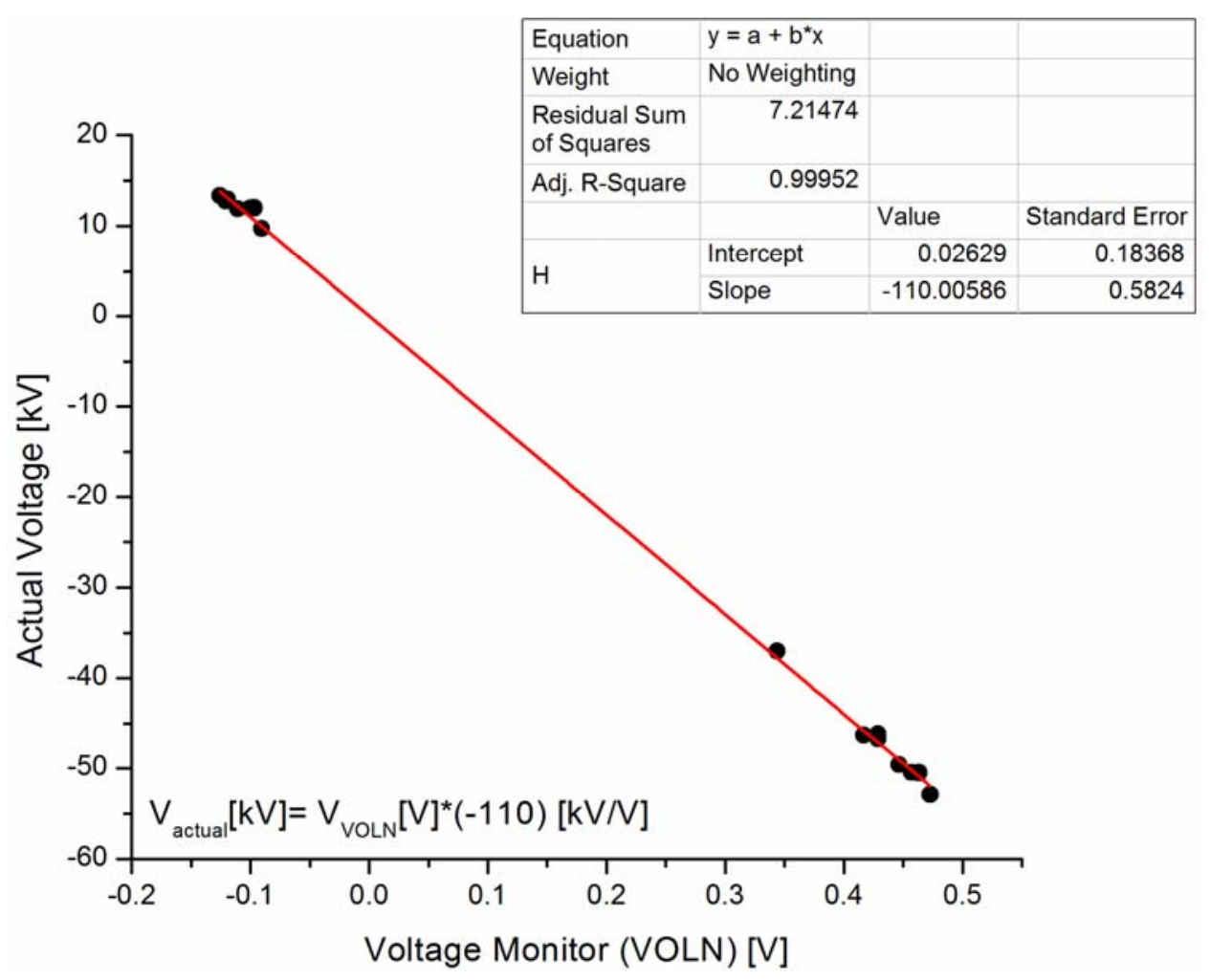

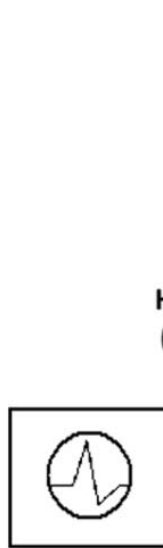

H/V Pulser
Local Oscilloscope

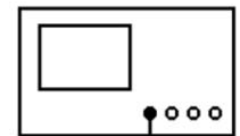

$(1000 \times 1$

$(1000 \mathrm{X})$

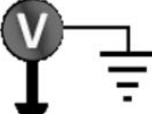

\section{Screen Room Oscilloscope}

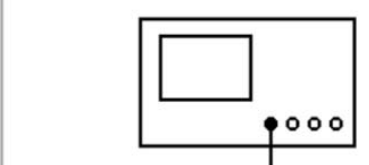

50 Ohm Terminator

10X Attenuator

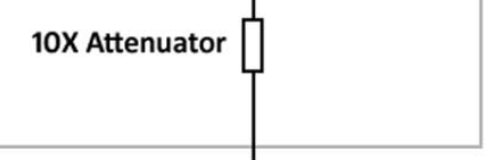




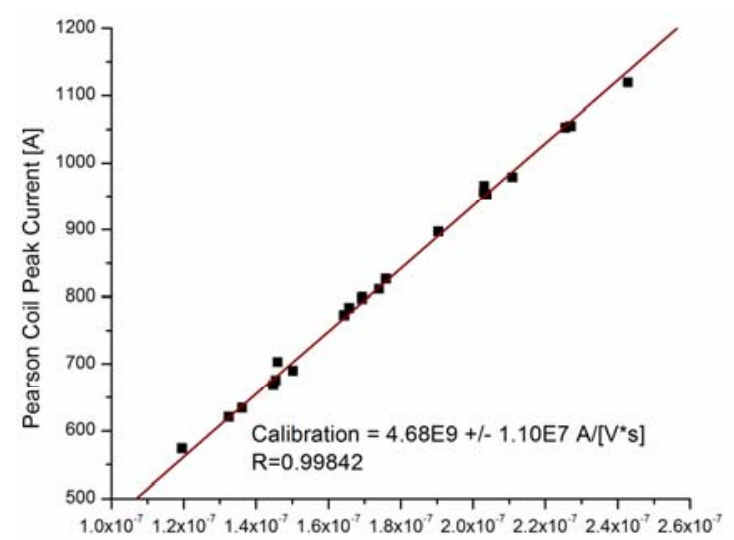

Magnetron Entrance Current Rogowski Peak of Integrated Voltage [V*s]]
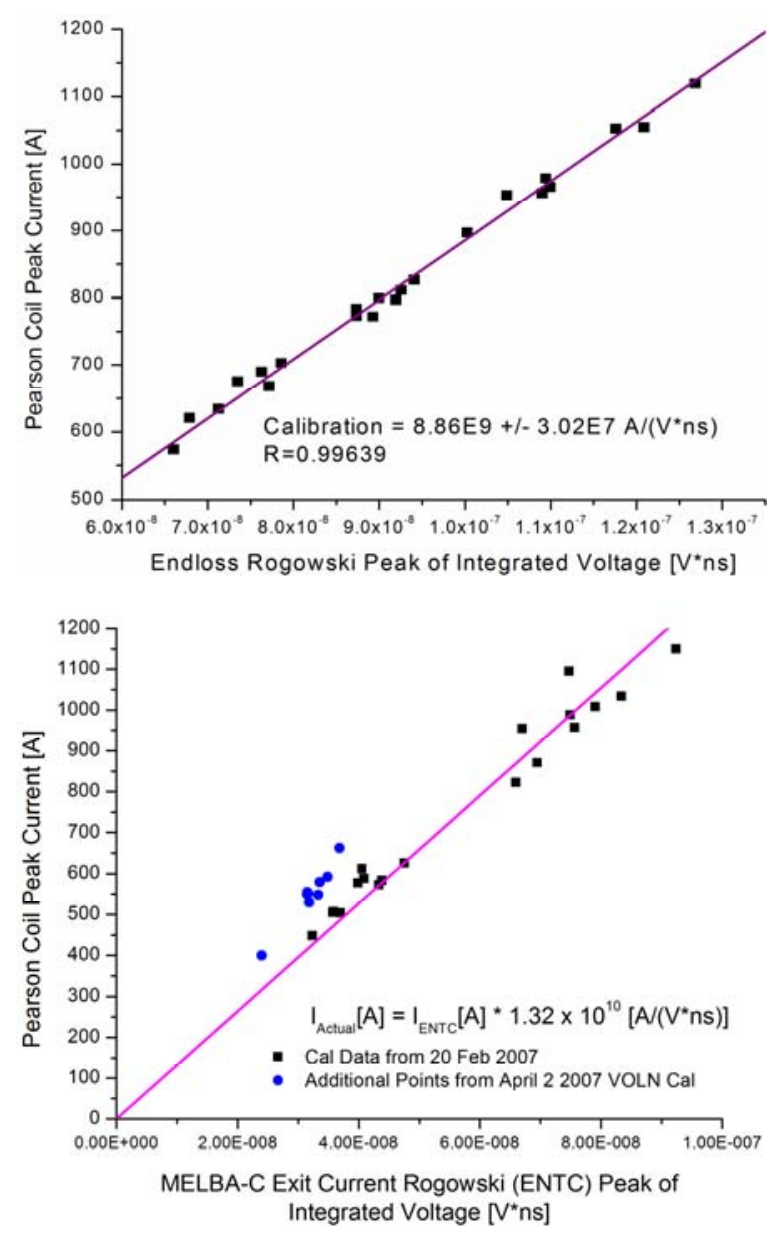

All Rogowski coils were calibrated as installed in the experiment. The cables from each of the Rogowski coils were terminated through a $10 \mathrm{X}$ attenuator and a $50 \Omega$ terminator into the oscilloscope. All components (coils + cables + attenuator + terminator) were taken into account by the calibrations. 

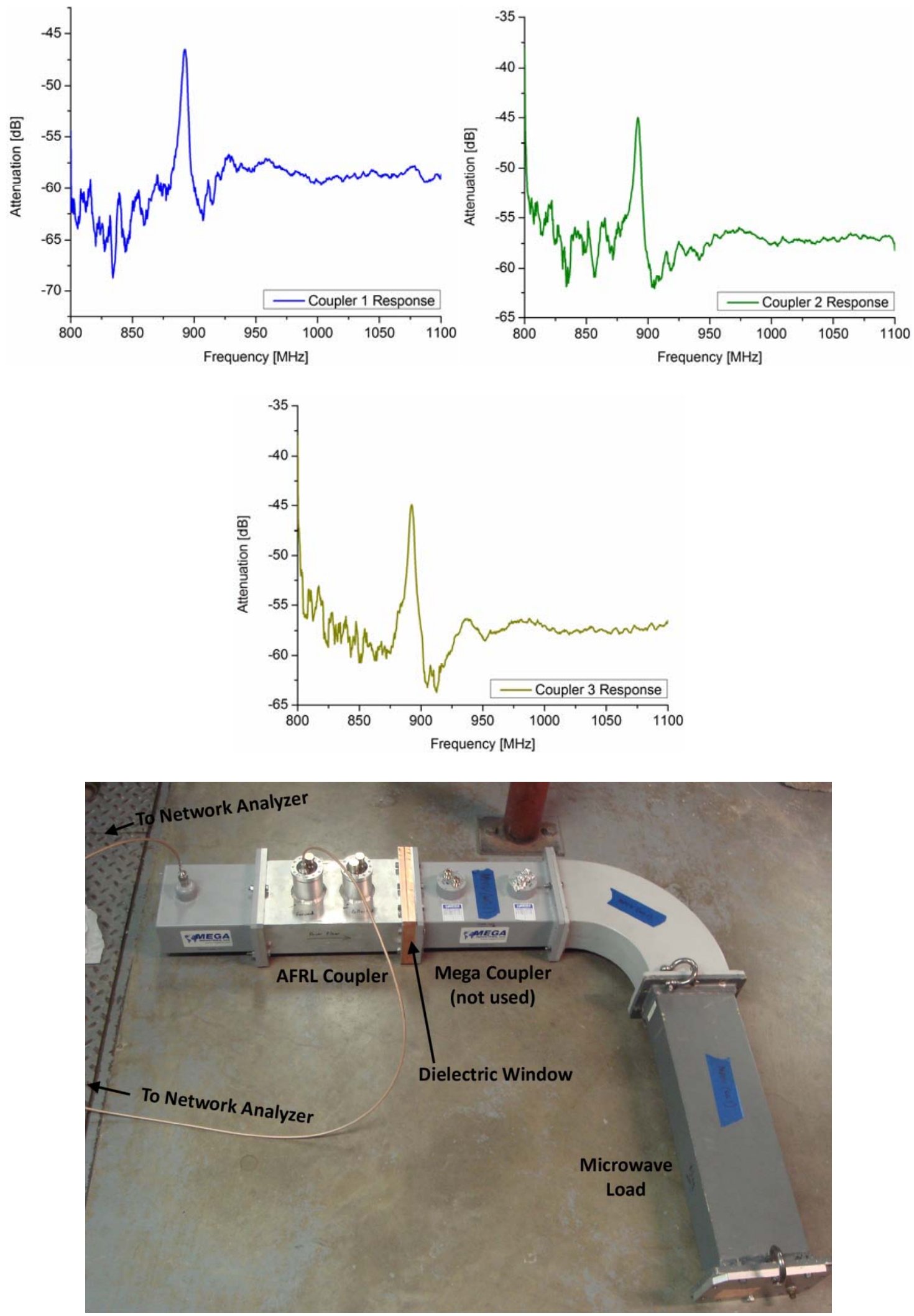

Calibrations for the AFRL microwave power couplers (configured to measure forward power from the forward set of terminals). 

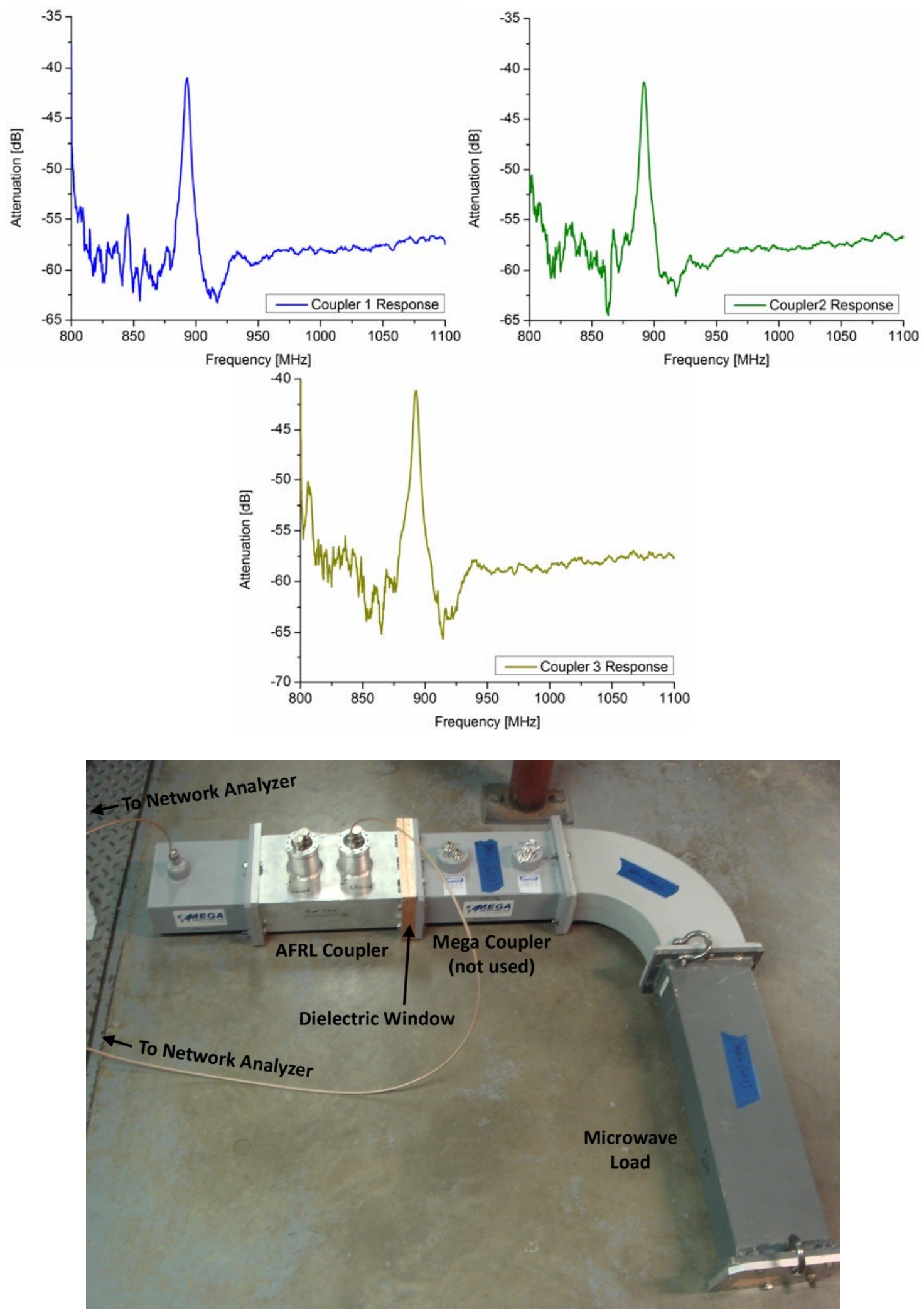

Calibrations for the AFRL microwave power couplers (configured to measure forward power from the reverse set of terminals). 

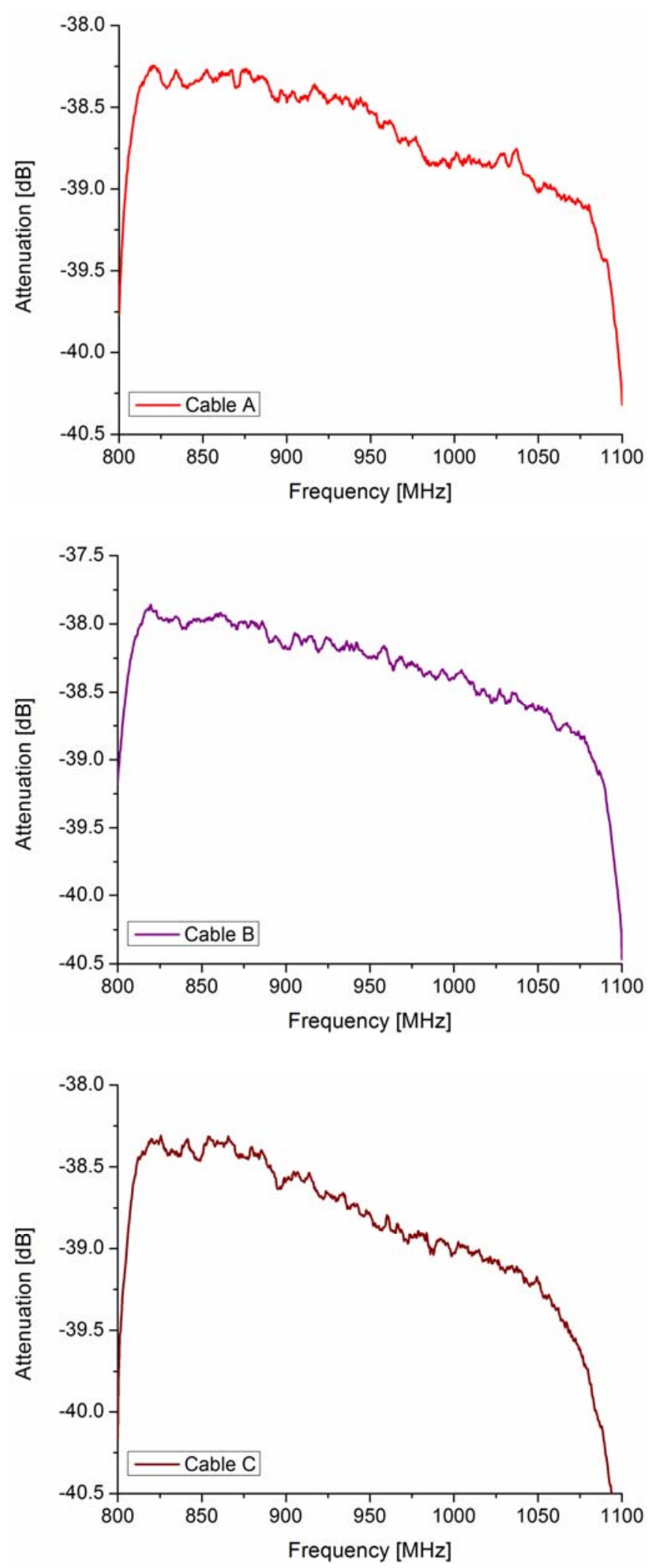

Attenuation profiles for cables A, B, and C. These profiles include the cable itself plus any attenuation and filtering attached to the cable. 

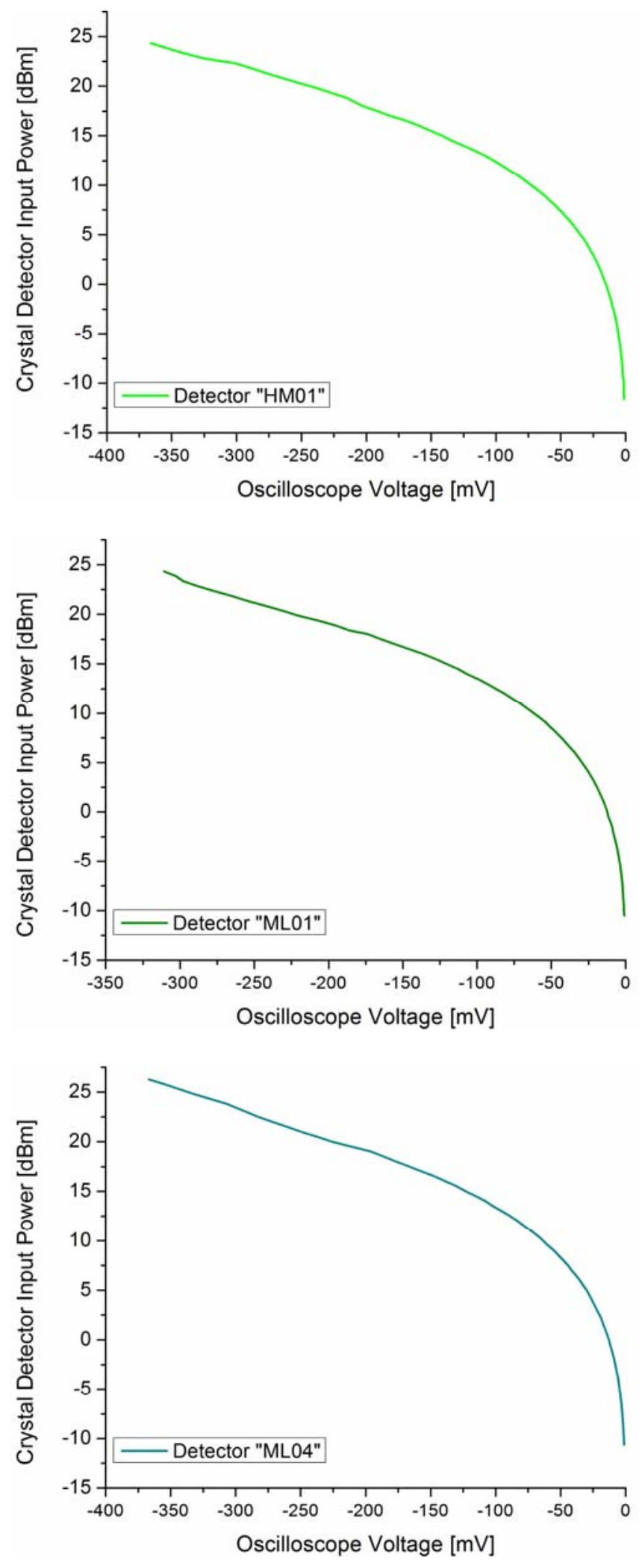

Diode detector response profiles. 


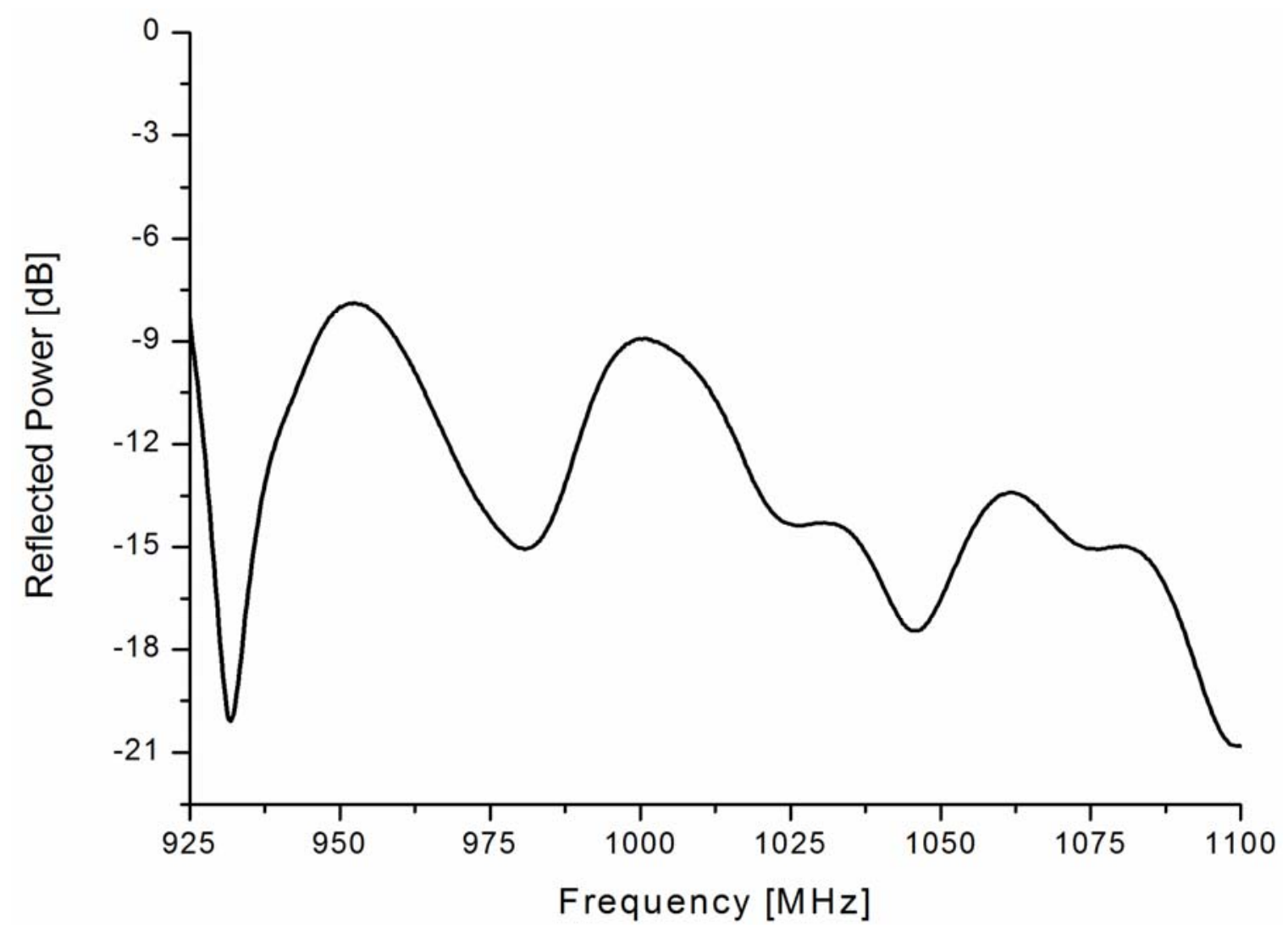

WR650 Eccosorb microwave load response. 


\section{Appendix B}

Equipment Technical Drawings and Pictures

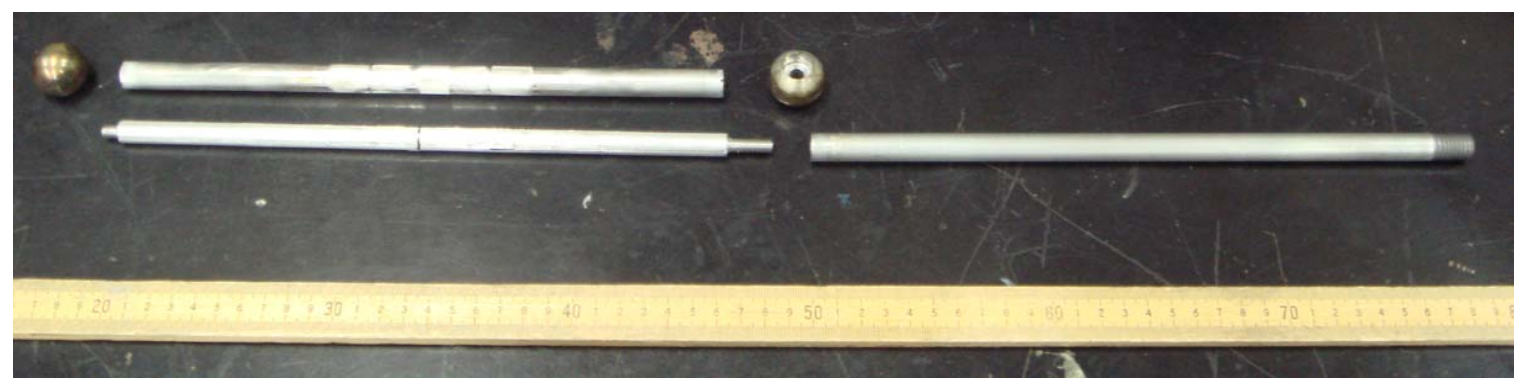

Disassembled magnetic priming cathode.

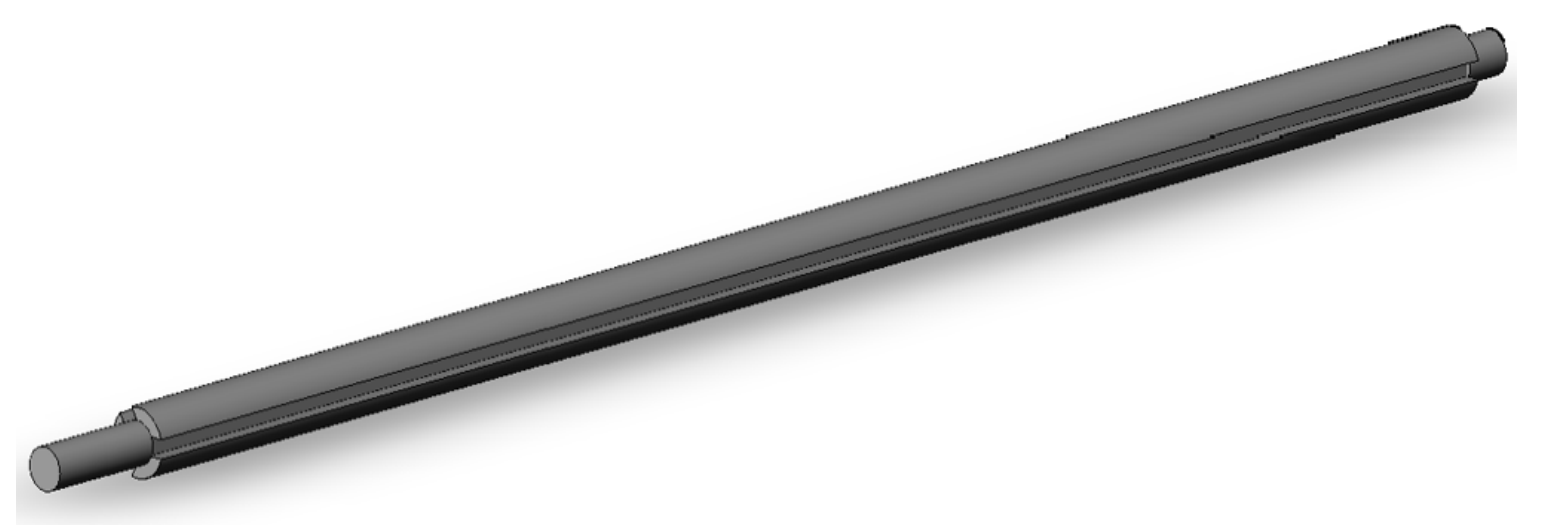

Grooved cathode tensioning rod. 

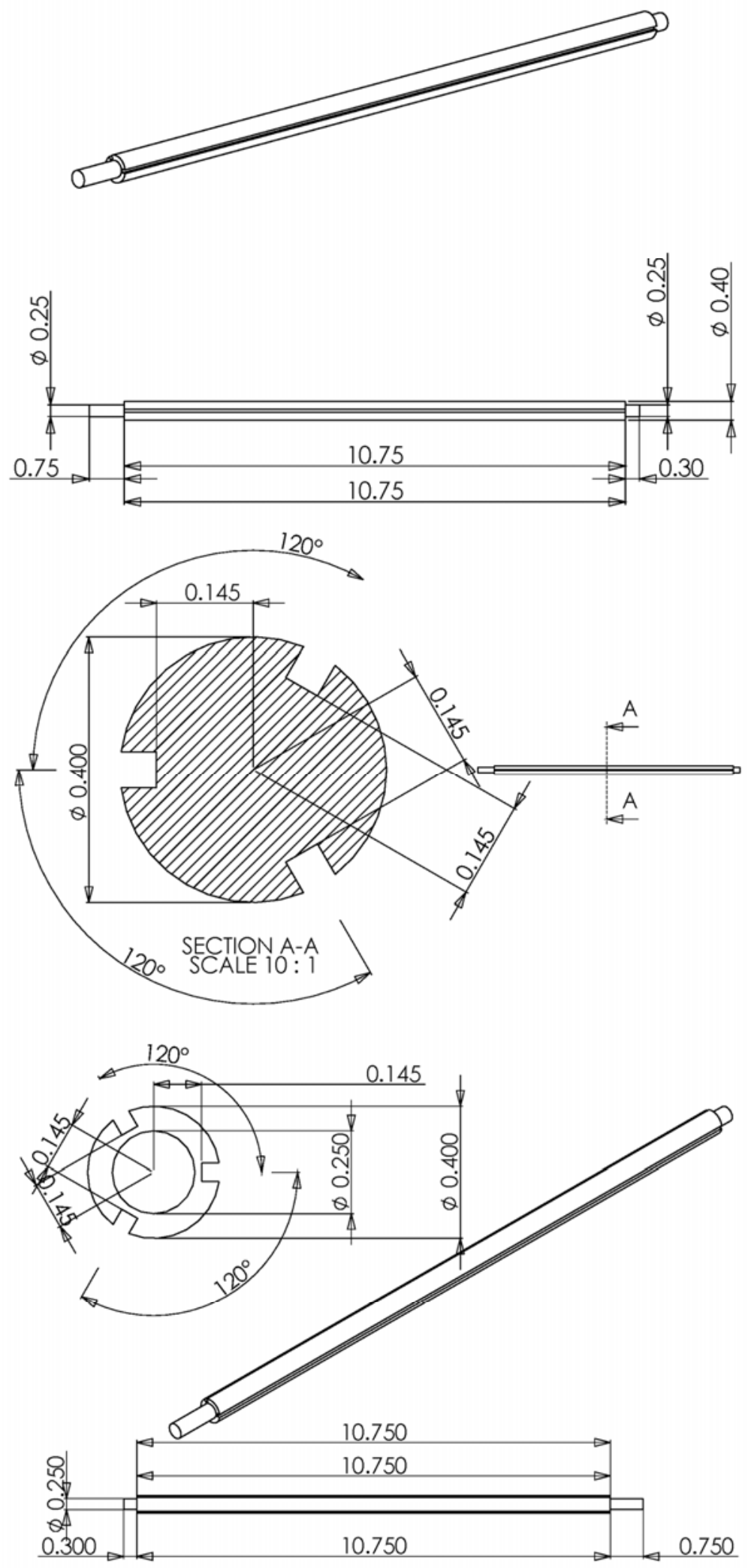

Cathode tensioning rod with grooves for $0.127 \mathrm{~cm}$ diameter wires. 

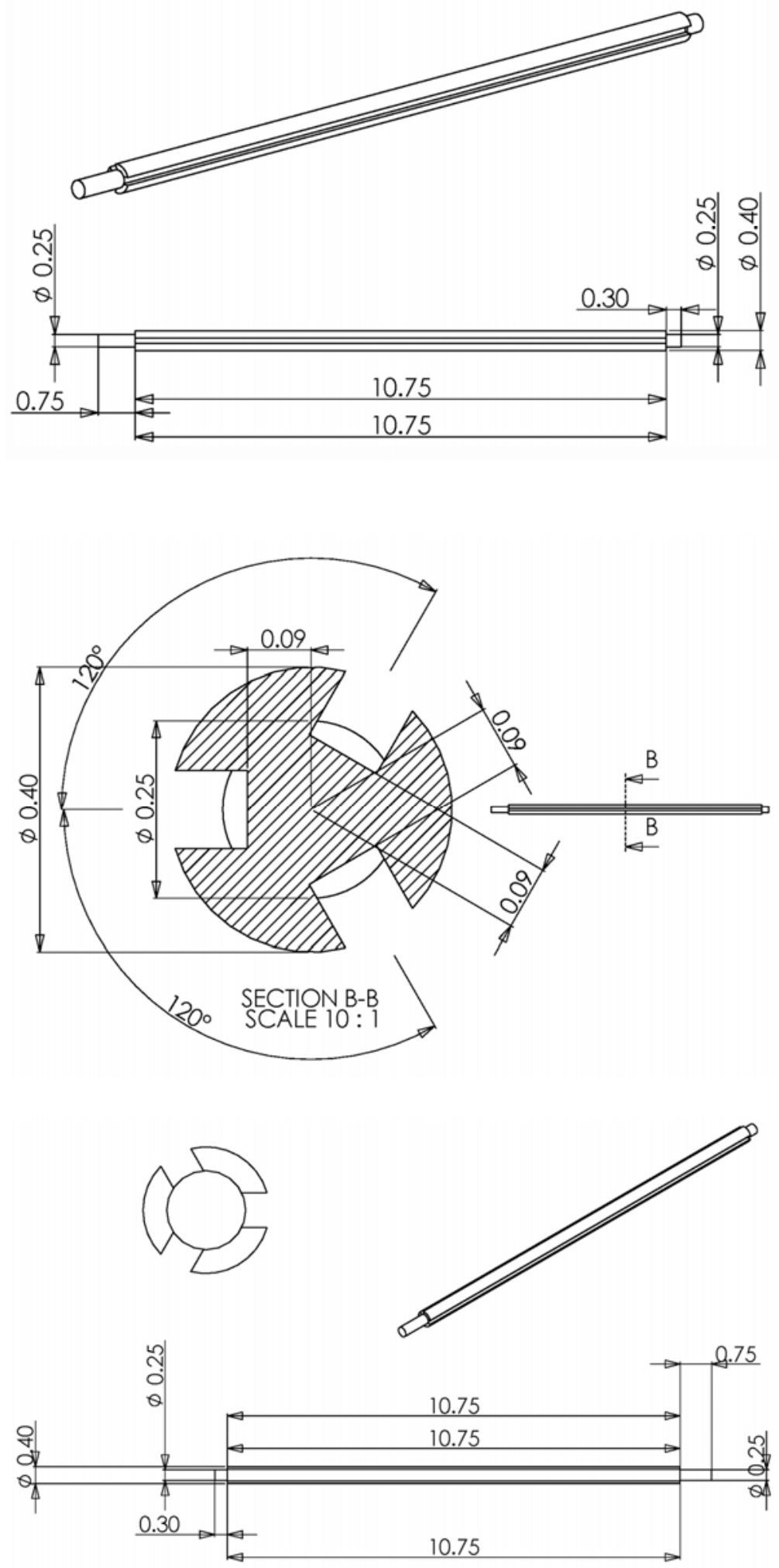

Cathode tensioning rod with grooves for $0.254 \mathrm{~cm}$ diameter wires. 

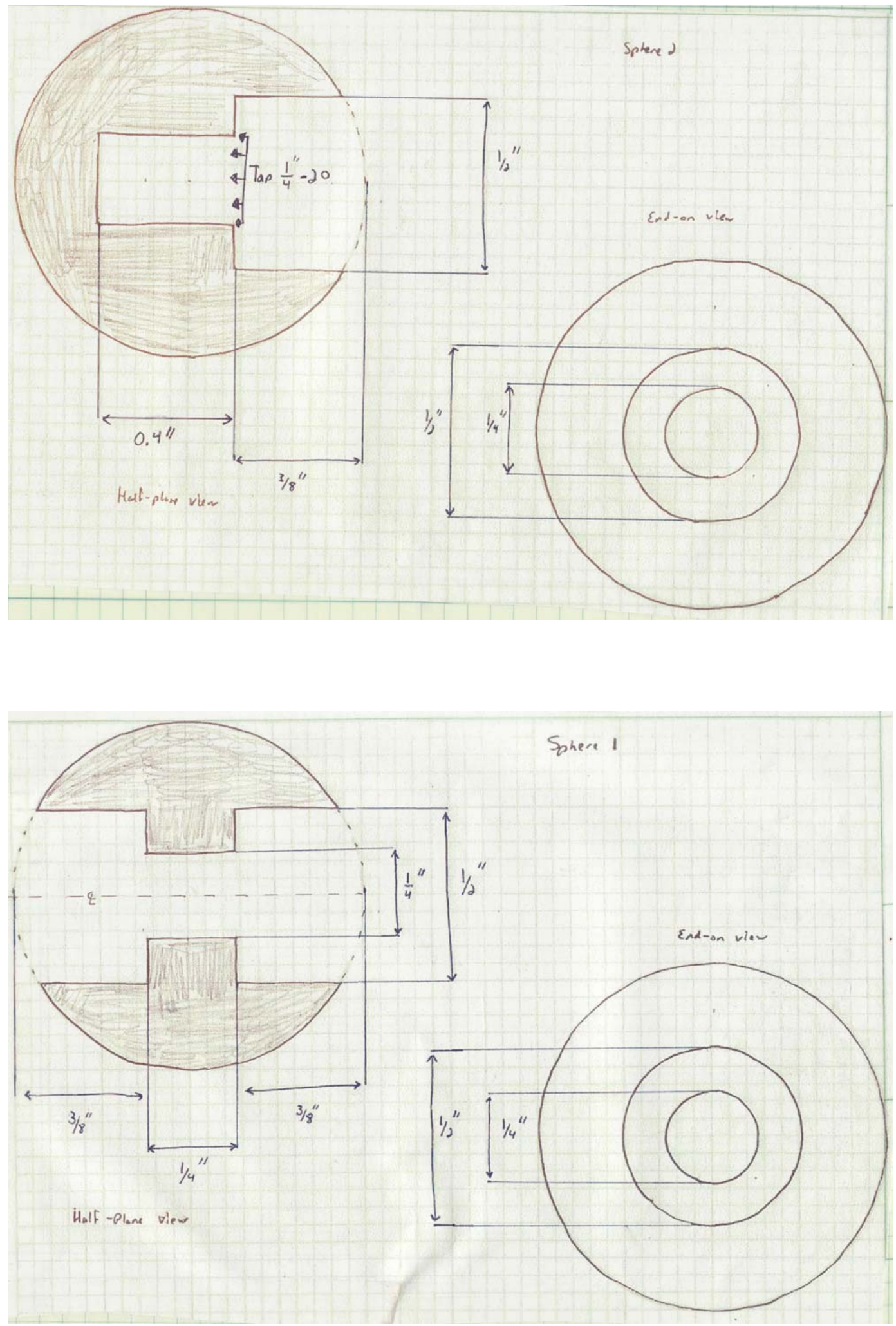

Design sketches for the magnetic priming cathode electrostatic end balls. 

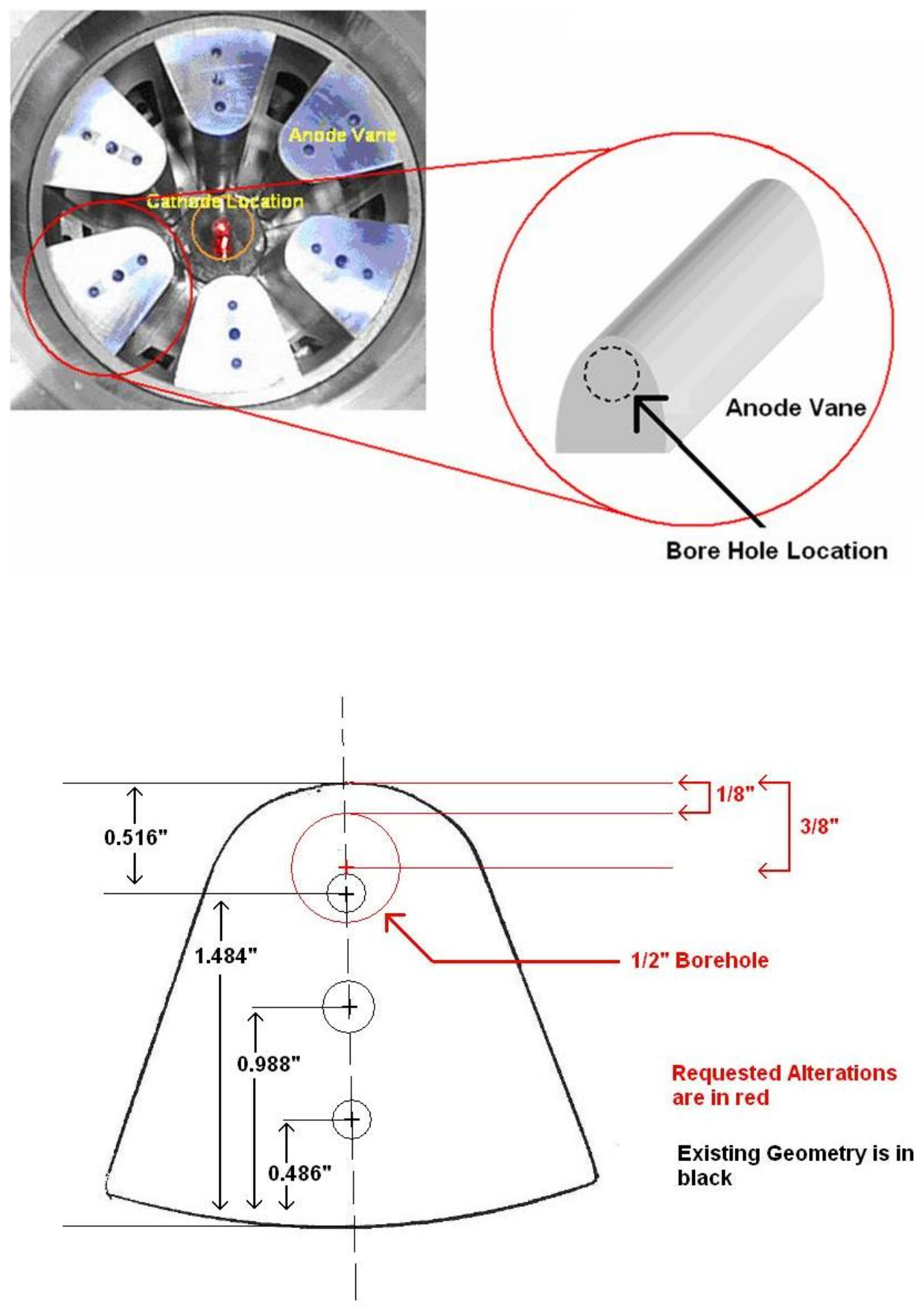

Anode Vane Modifications. 


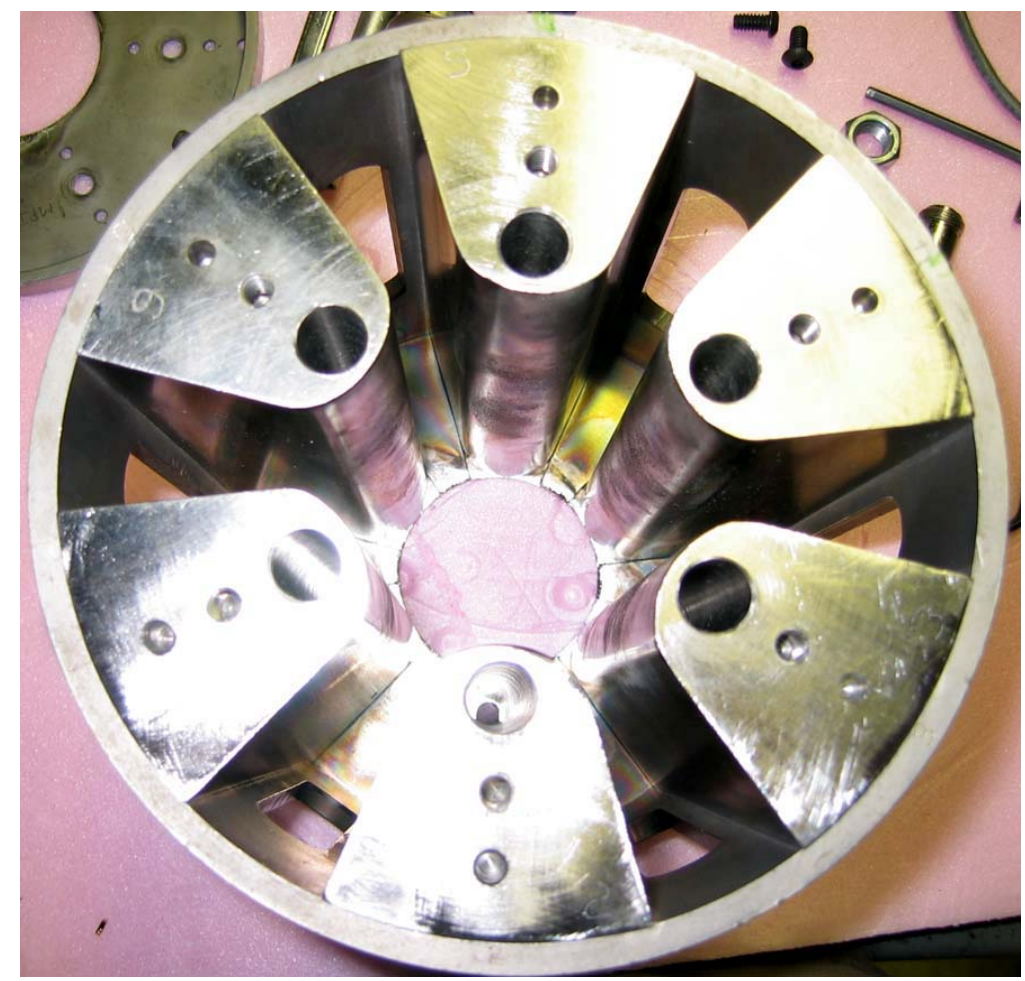

Anode vanes and support cylinder (outer diameter $=17.8 \mathrm{~cm}$ ).

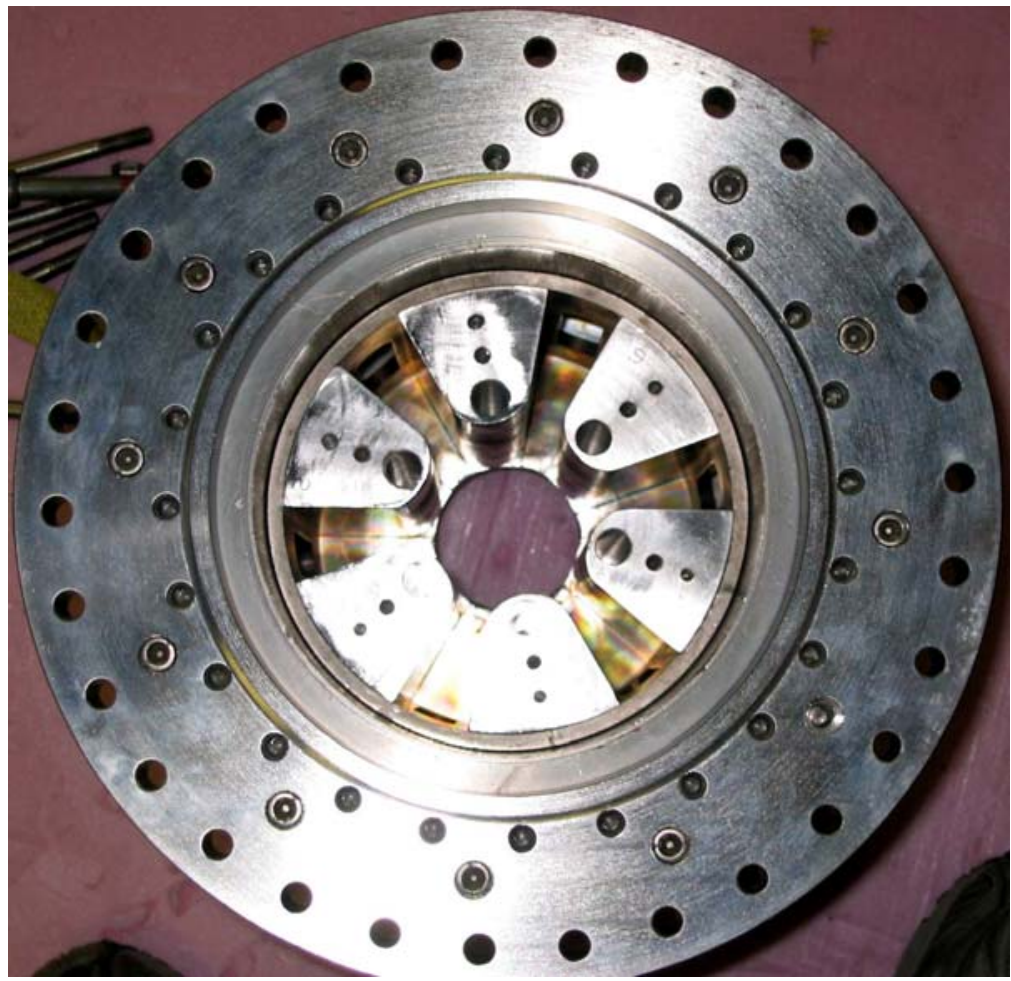

Anode vanes and support cylinder installed in vacuum housing. 

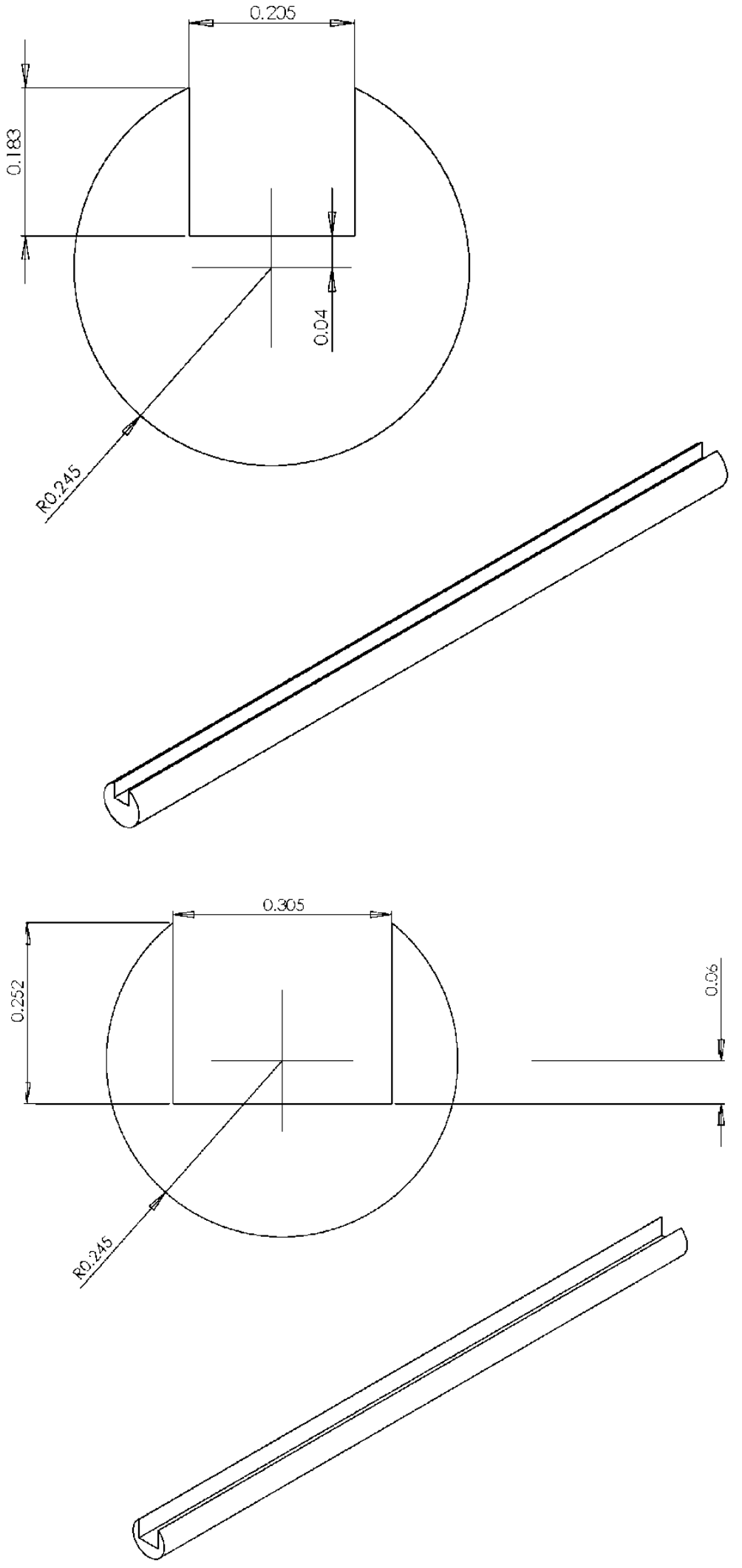

Anode wire support rods.

145 


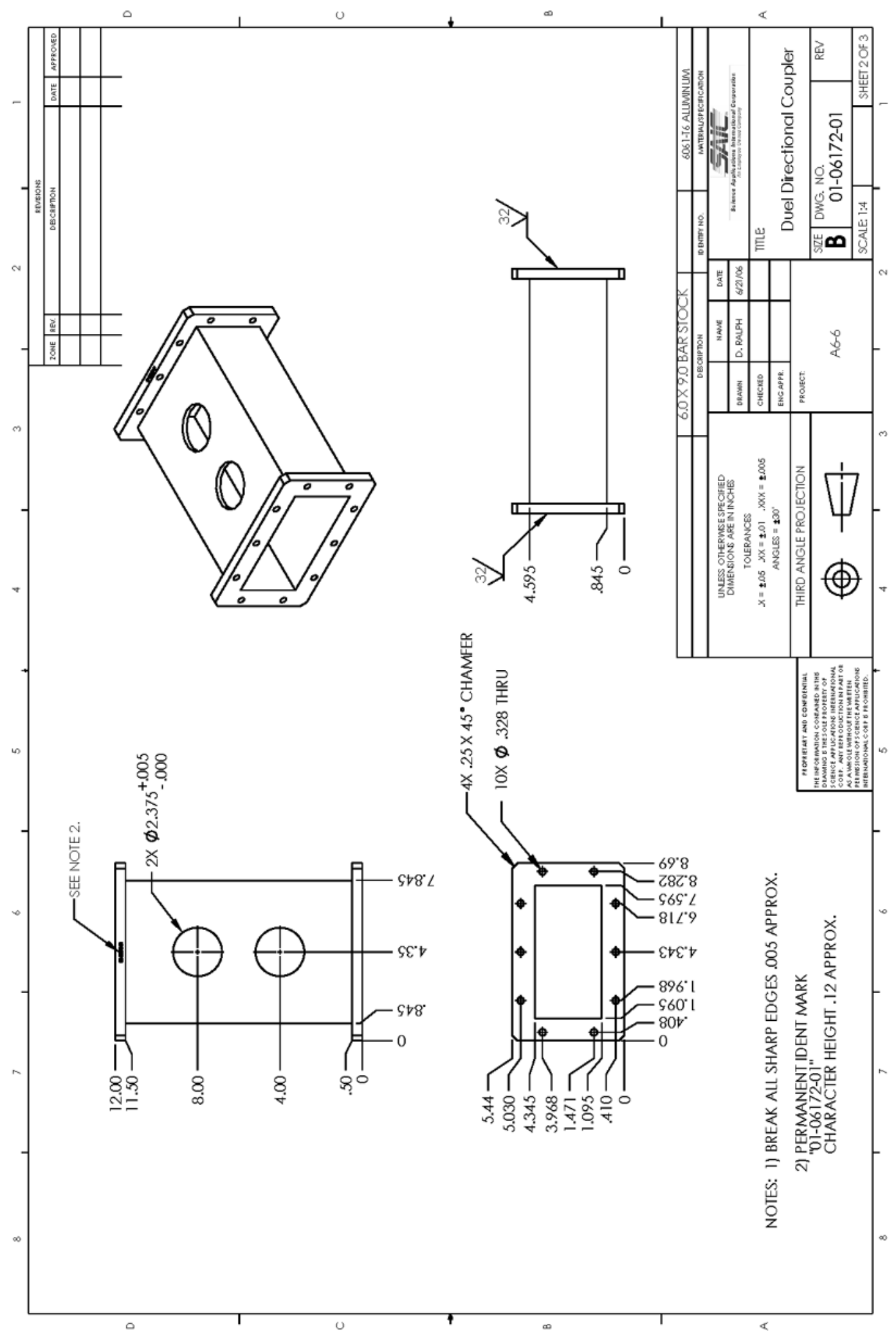

Vacuum rated power coupler, designed by M. D. Haworth of Air Force Research Laboratories (units in inches). 


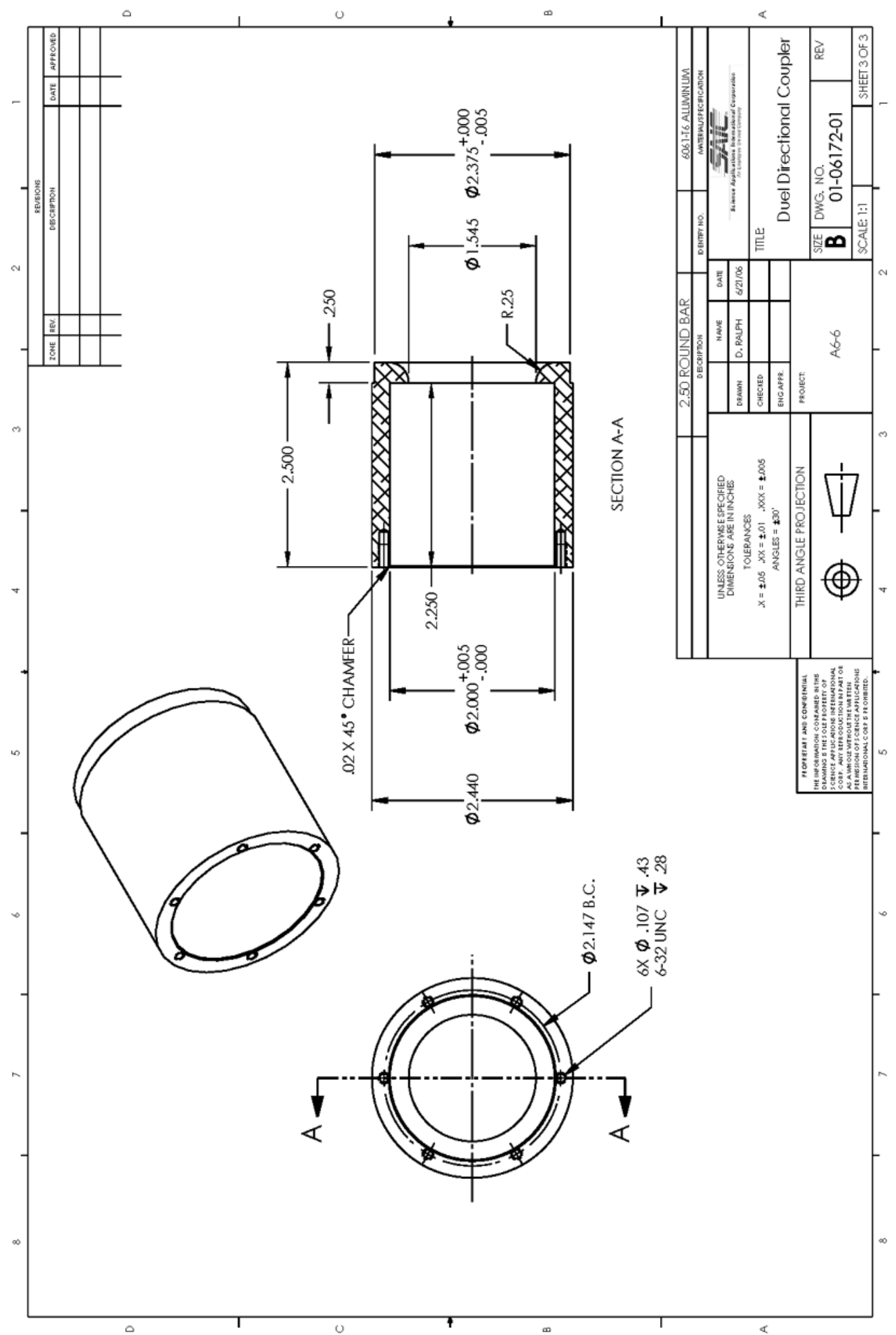

Vacuum rated power coupler, designed by M. D. Haworth of Air Force Research Laboratories (units in inches). 


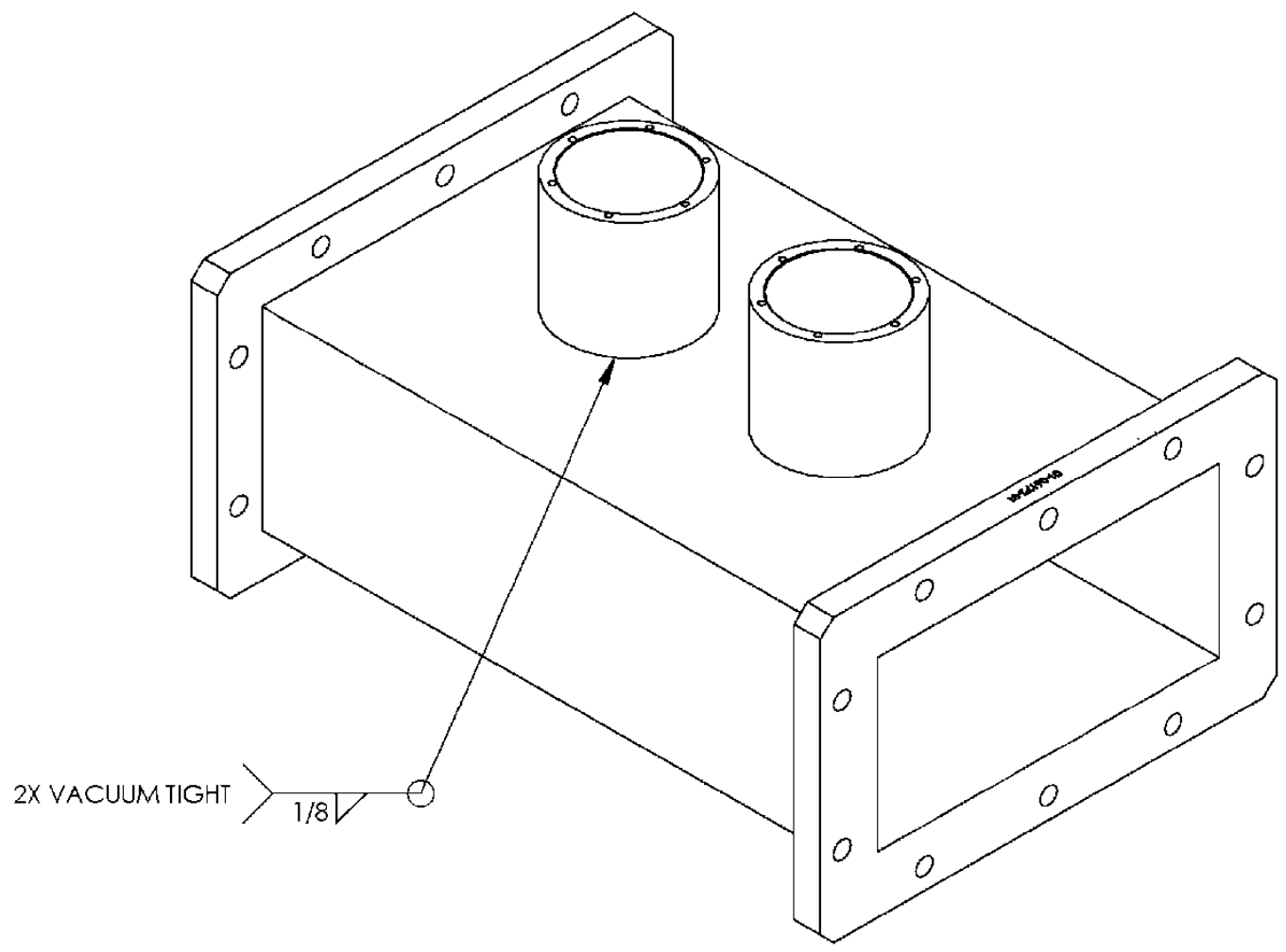

Vacuum rated power coupler, designed by M. D. Haworth of Air Force Research Laboratories (units in inches). 

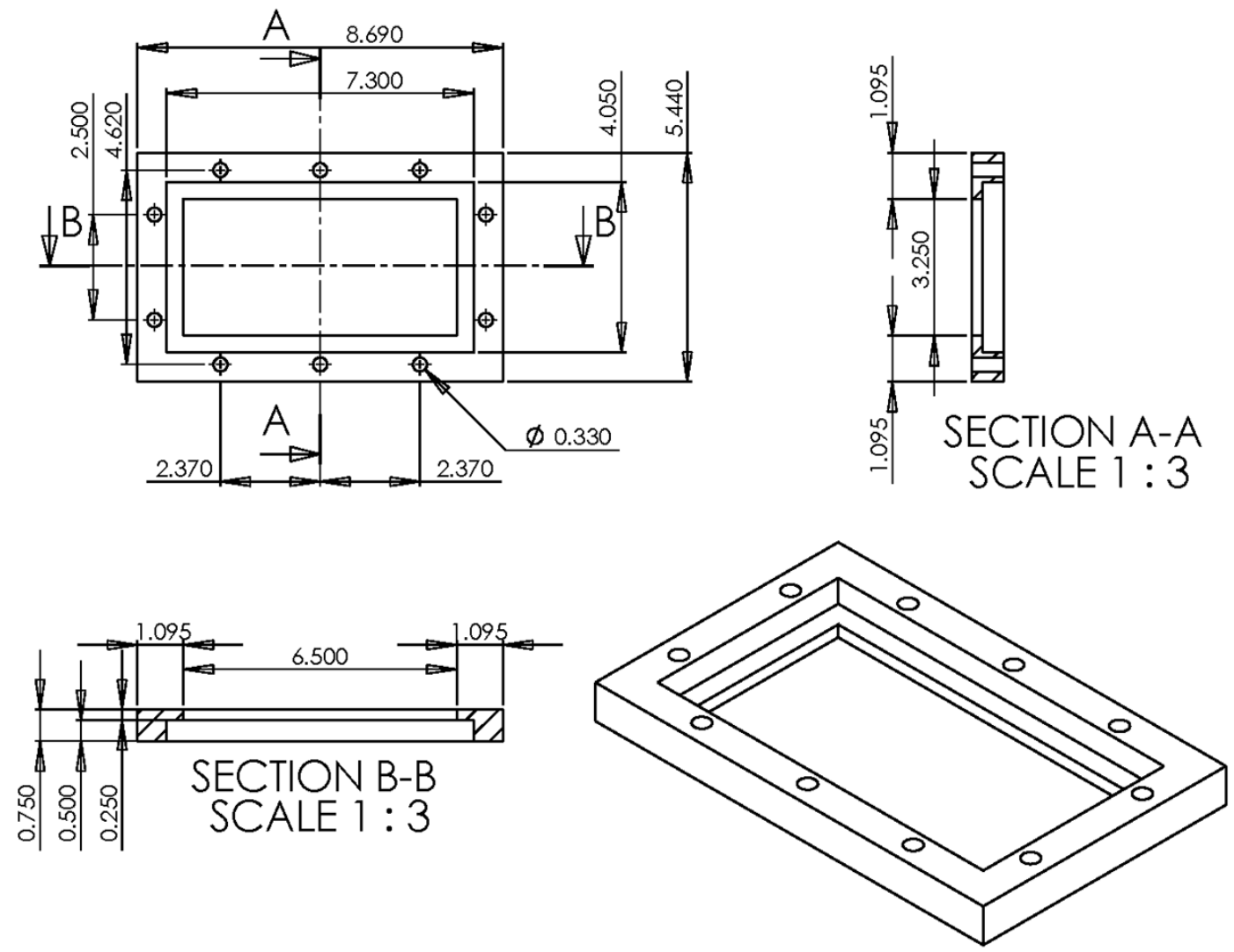

Microwave window frames for WR650 waveguide (units in inches). 


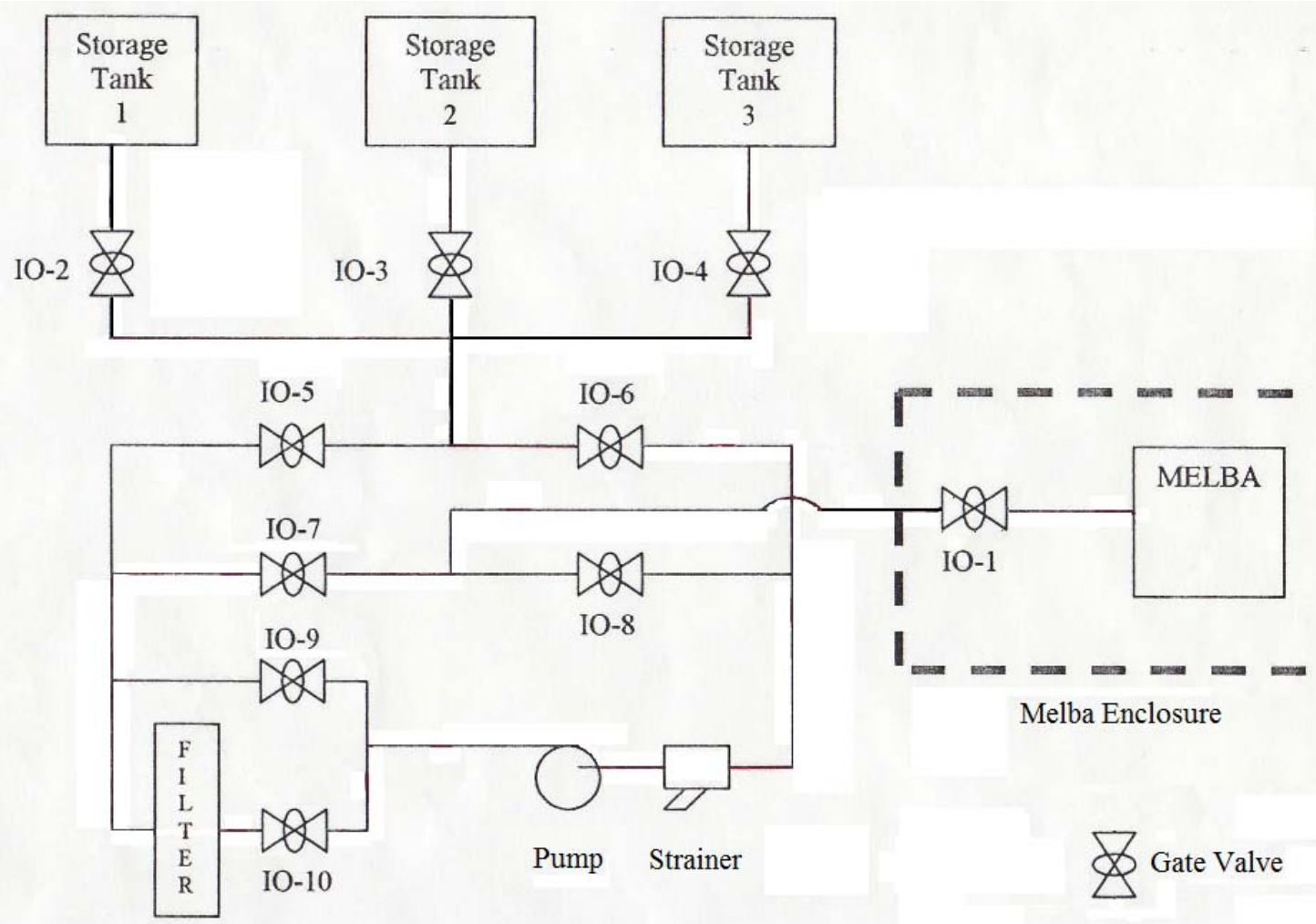

MELBA-C insulation oil handling system 


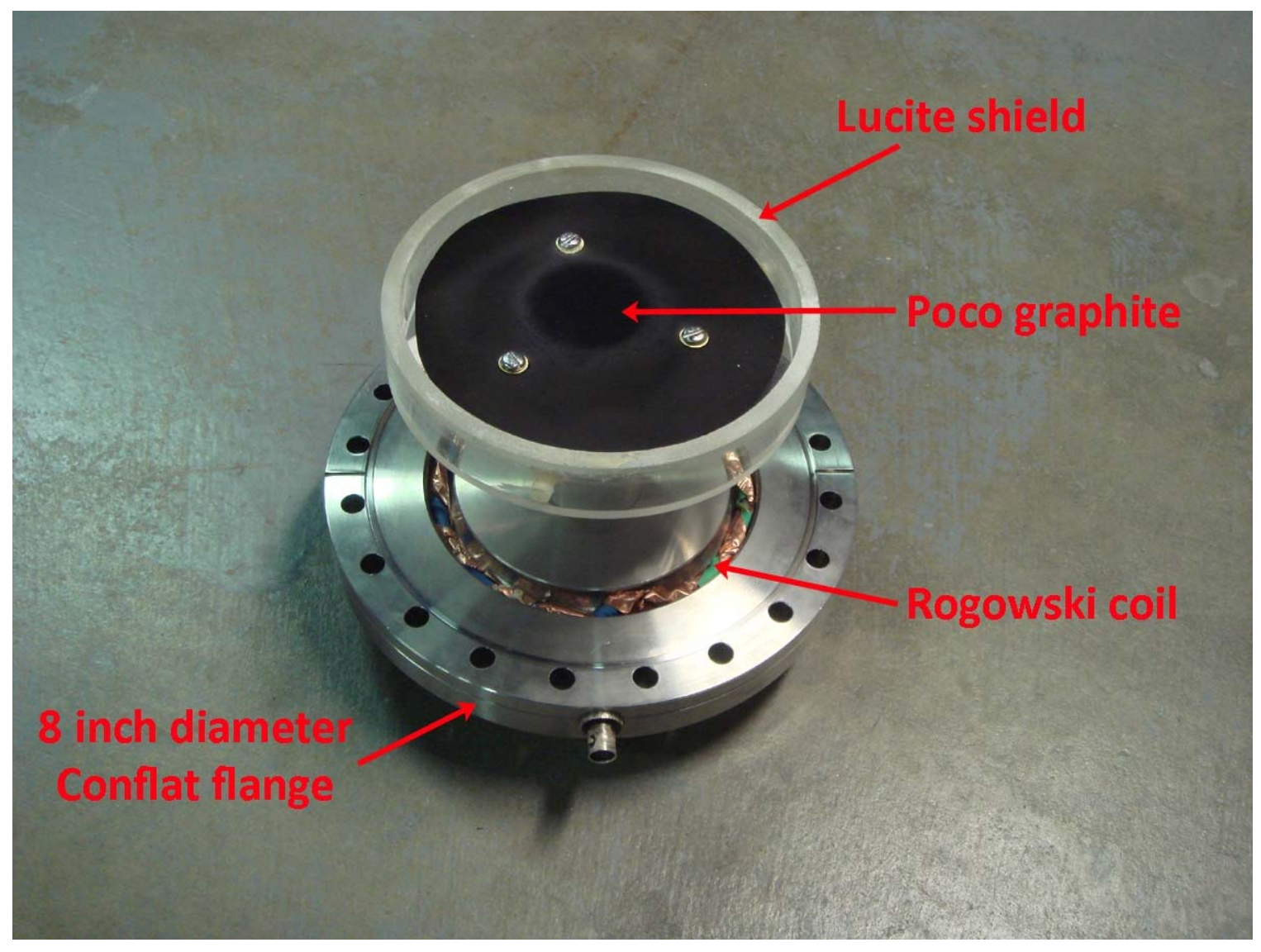

End loss current detector. The Poco graphite plate is mounted to a copper backing which then connects to the center of the flange via a $2 \mathrm{~cm}$ diameter copper rod. 


\section{Appendix C}

\section{Additional Magnetic Priming Data}
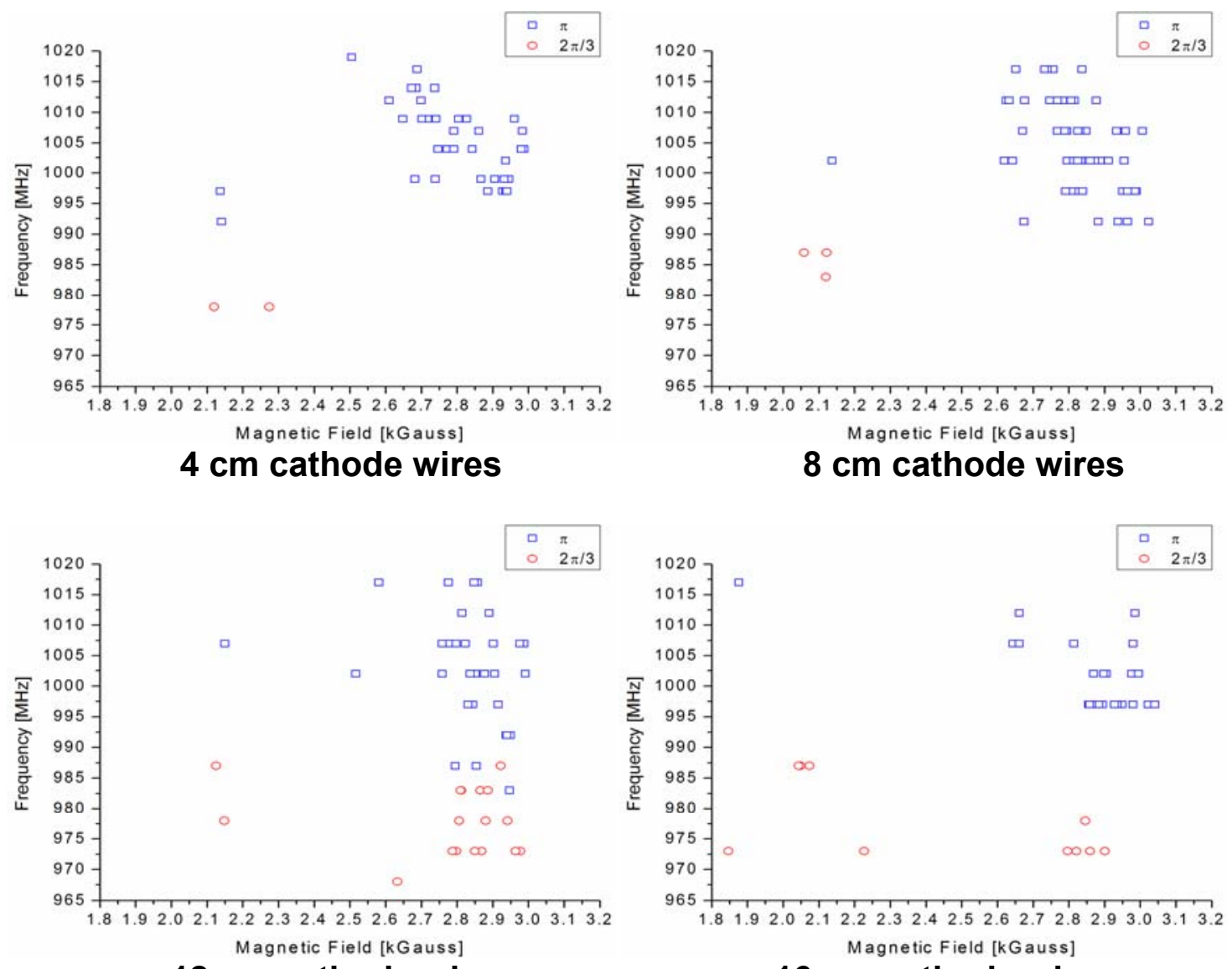

$12 \mathrm{~cm}$ cathode wires
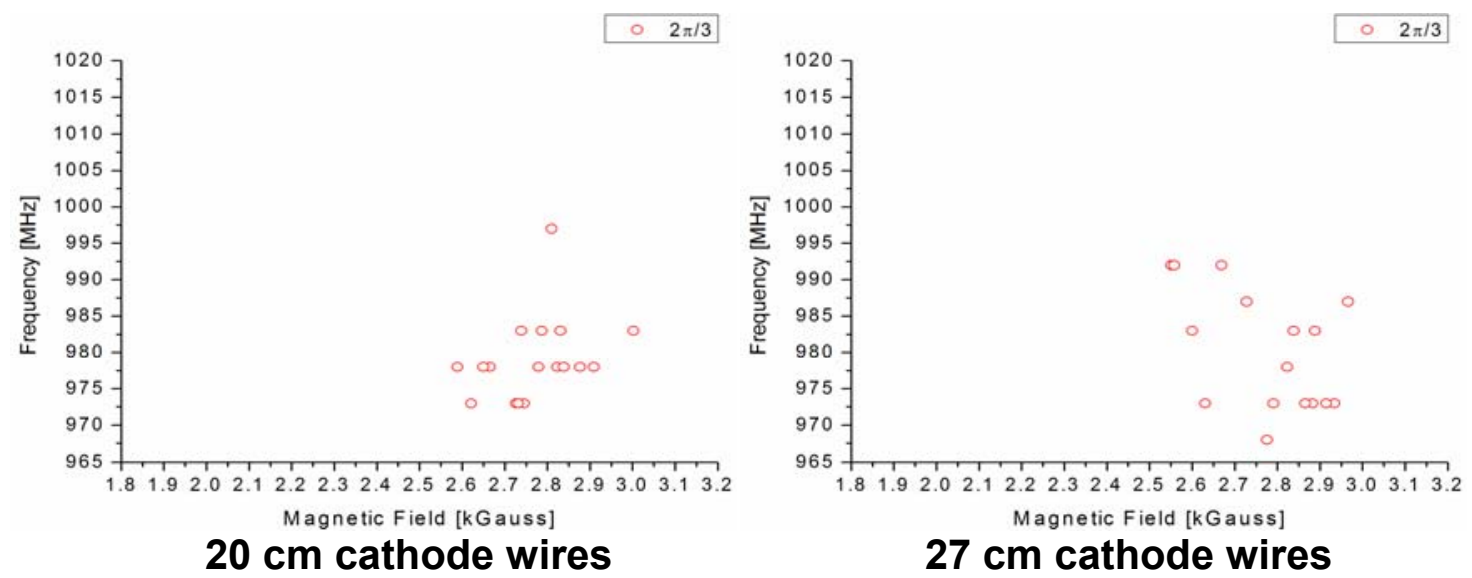

Frequency at peak power as a function of applied magnetic field for the cases of magnetic priming at the cathode (balanced loading) 


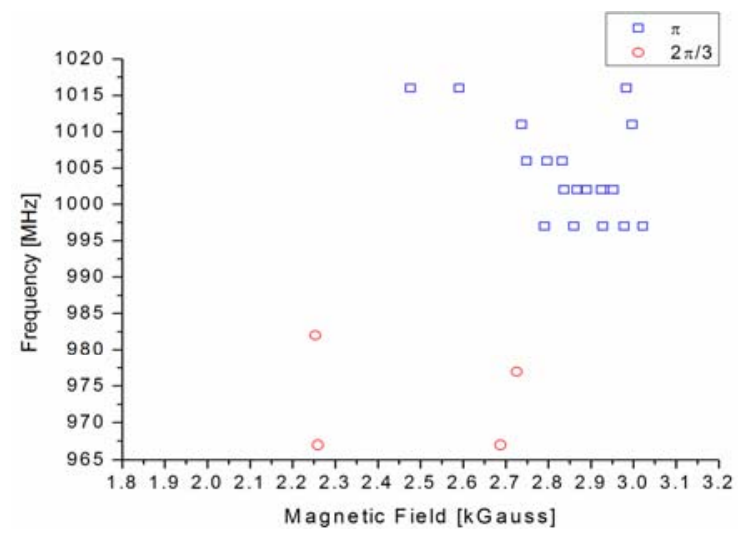

$10 \mathrm{~cm}$ anode wires

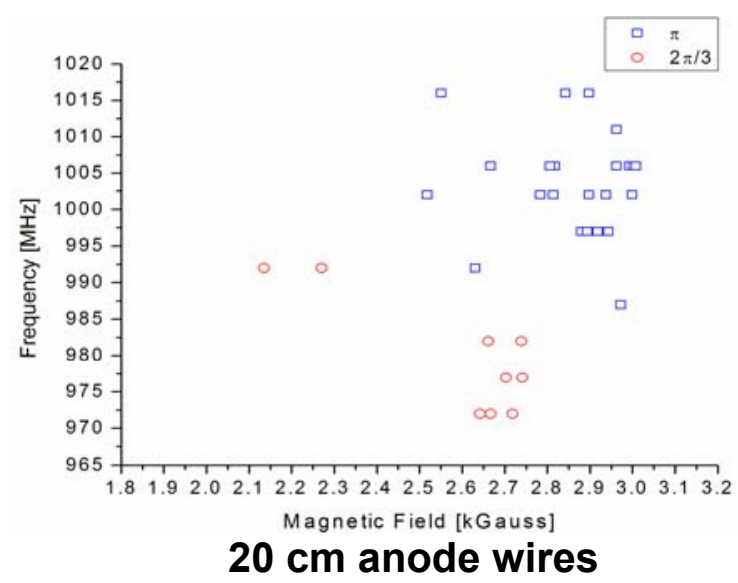

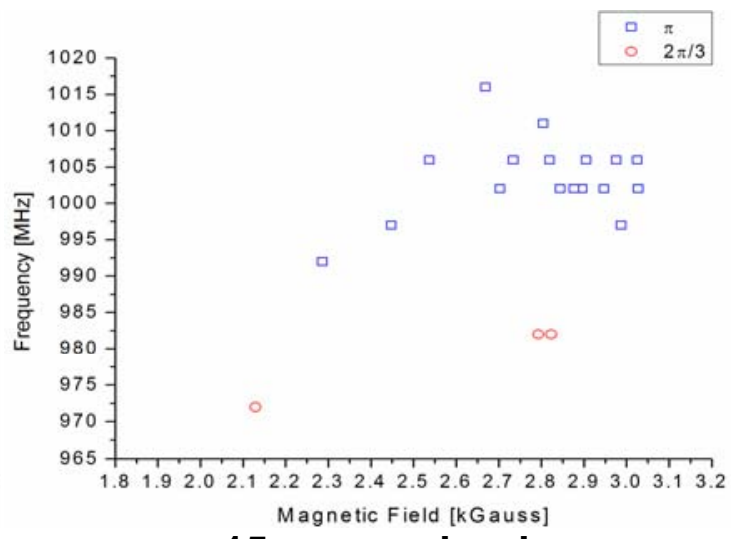

$15 \mathrm{~cm}$ anode wires

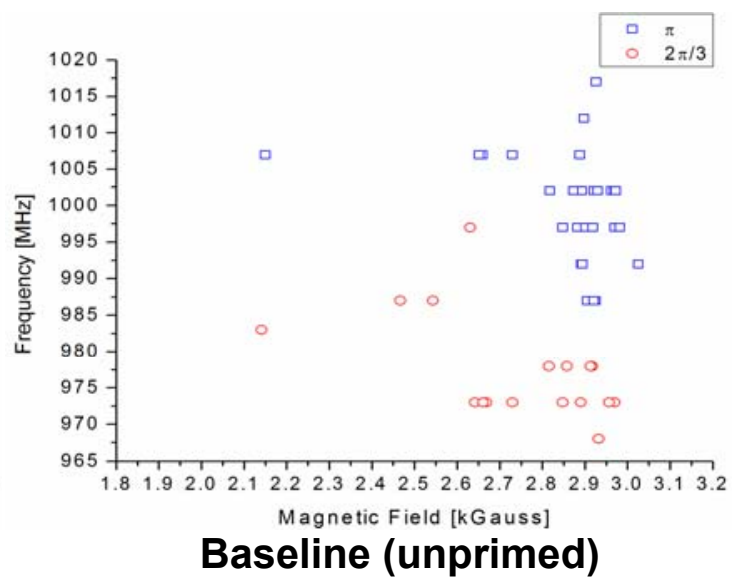

Frequency at peak power as a function of applied magnetic field for the cases of magnetic priming at the anode and the unprimed baseline case (balanced loading) 
Preliminary Findings for Combined Magnetic Priming at the Cathode and the Anode

Cathode wires $=6 \mathrm{~cm}$ long, $0.25 \mathrm{~cm}$ diameter.

Anode wires $=6 \mathrm{~cm}$ long, $0.76 \mathrm{~cm}$ diameter

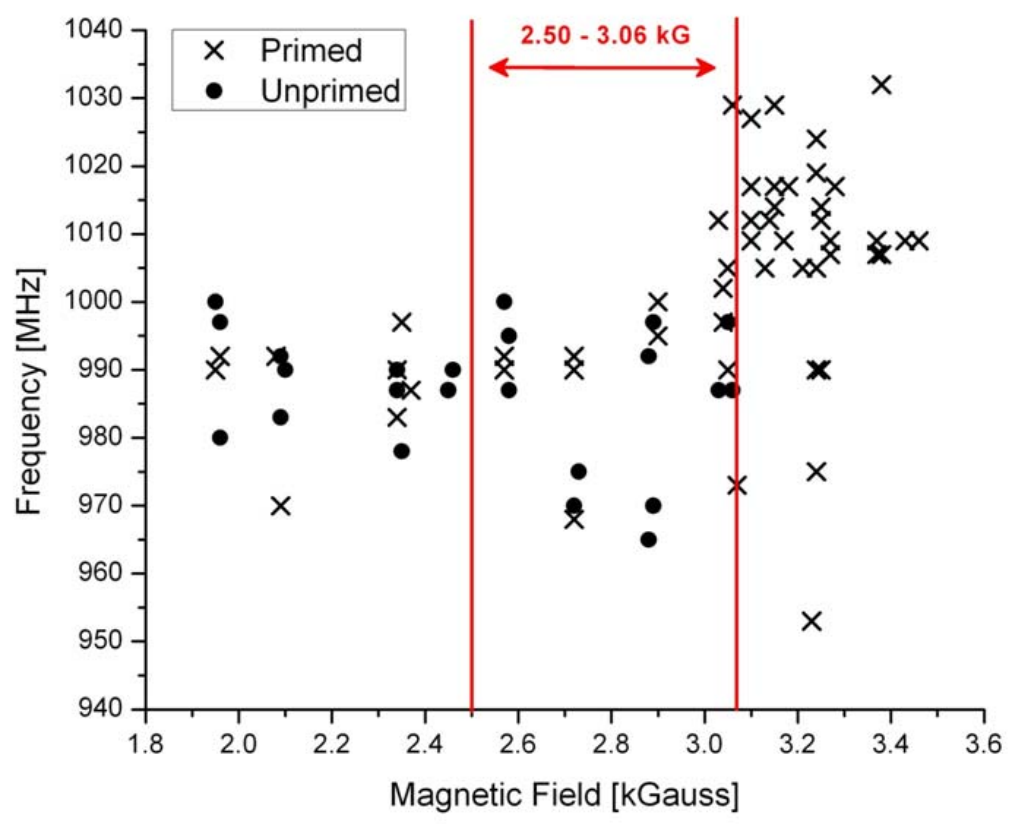

Magnetron frequency as a function of applied magnetic field.

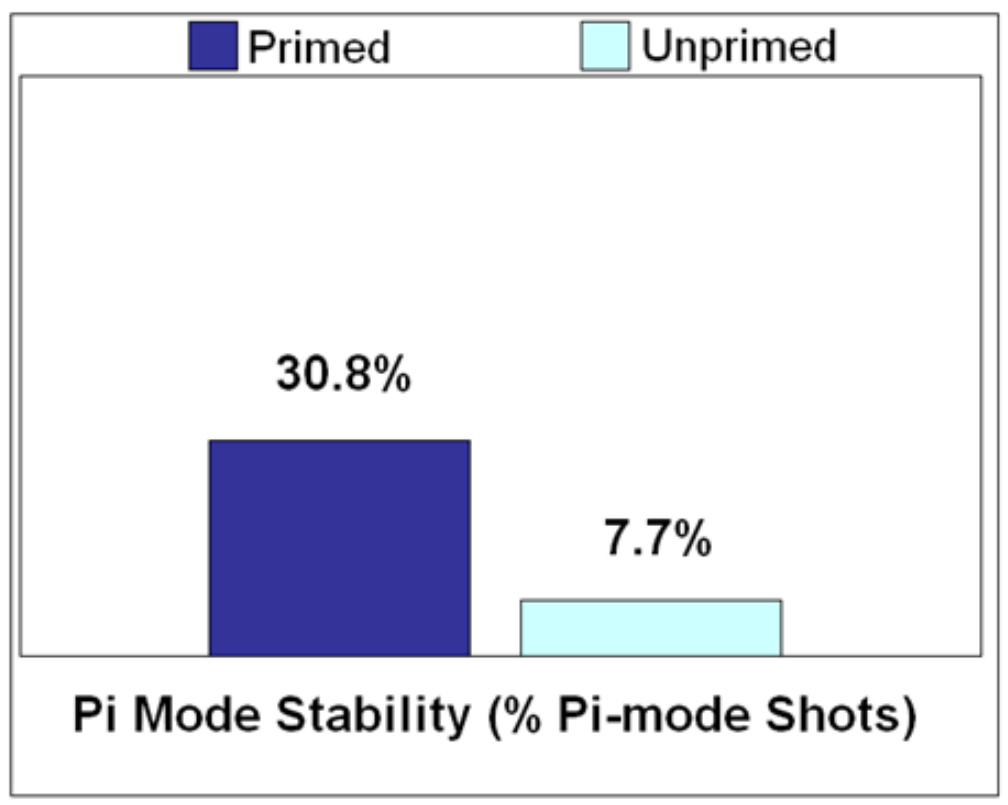




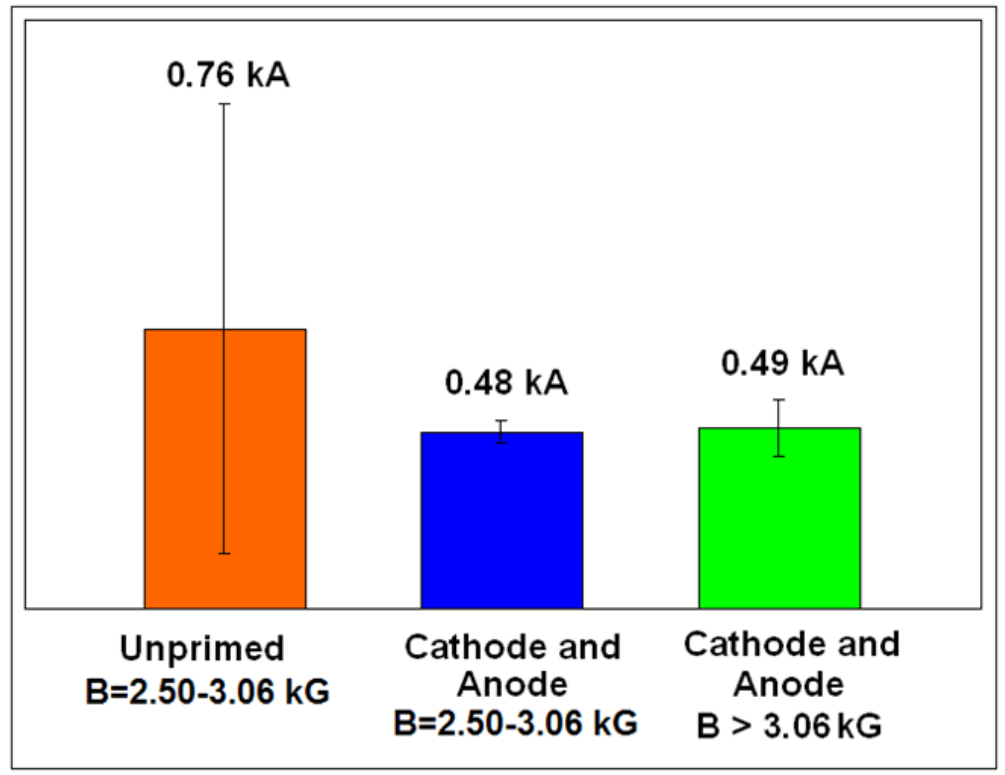

Starting current.

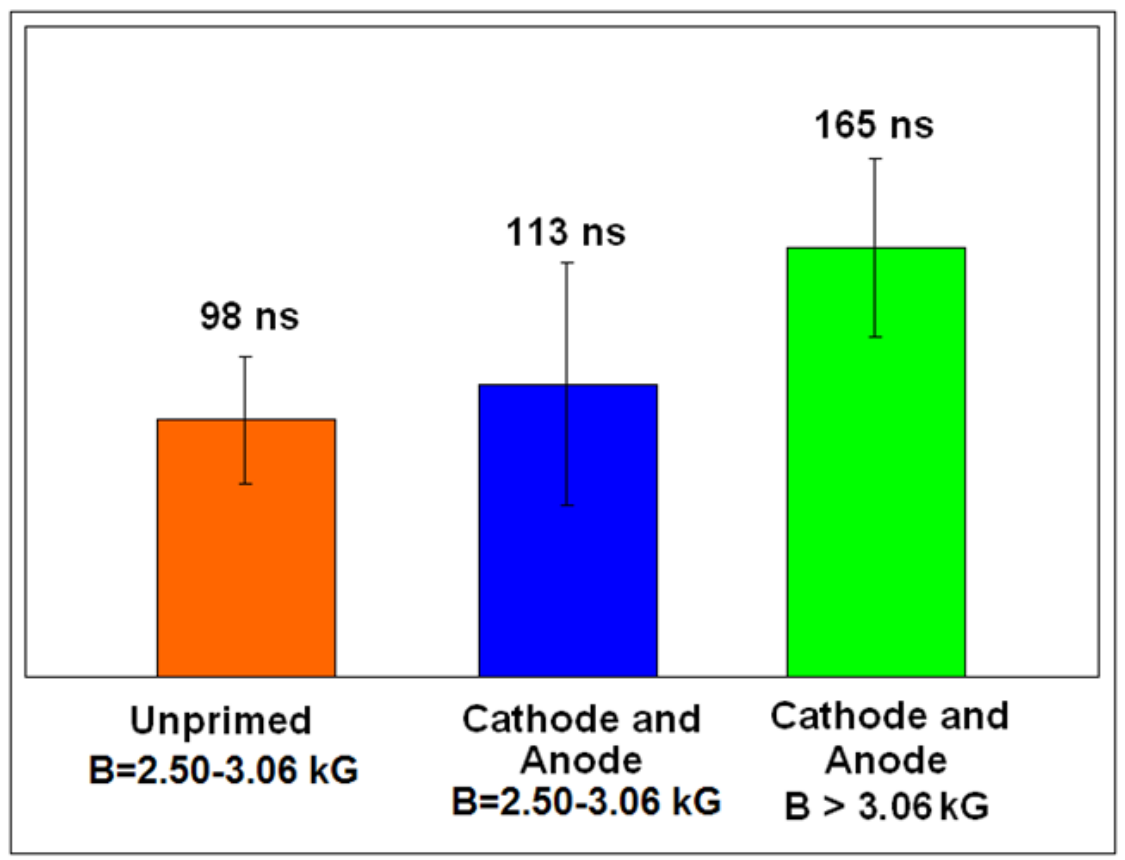

Pulse width. 


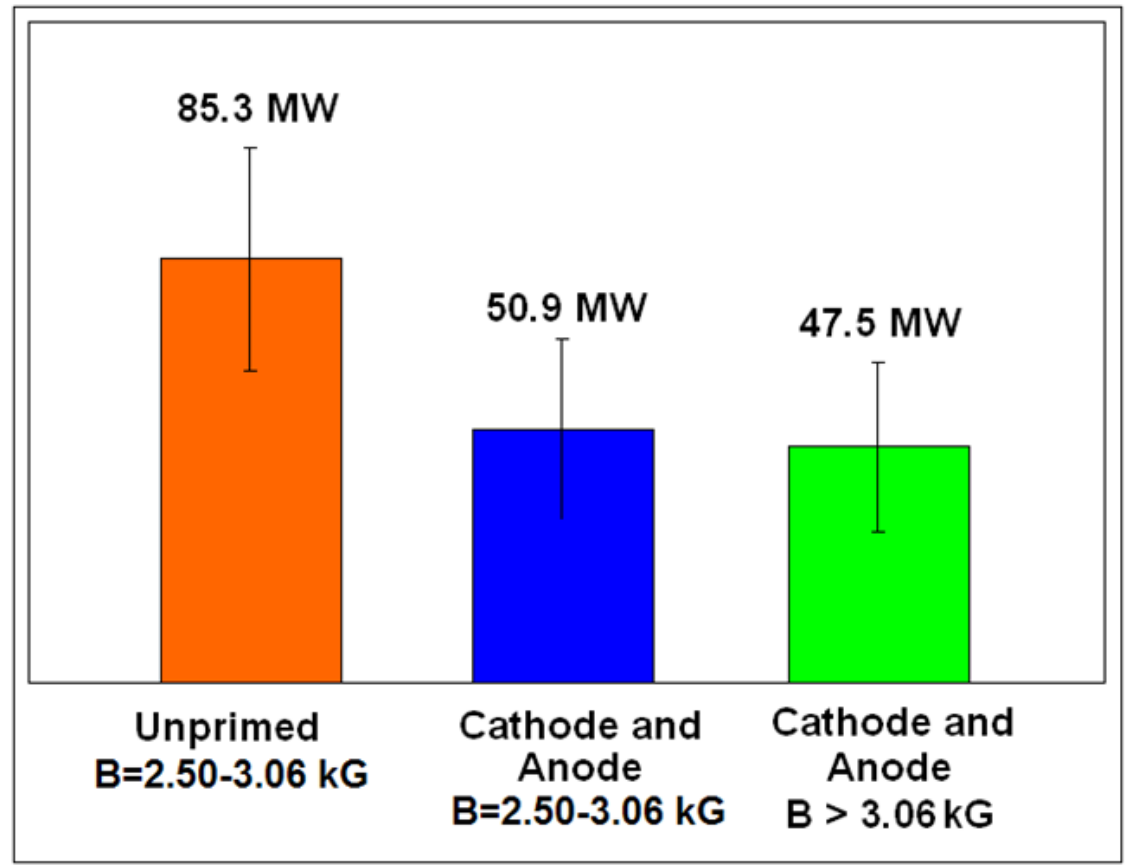

Peak power (3-waveguide combined). NOTE: these data were acquired in the old microwave window configuration, thus window flashing was likely occurring. 


\section{Appendix D}

\section{Experimental Cold Test Data}
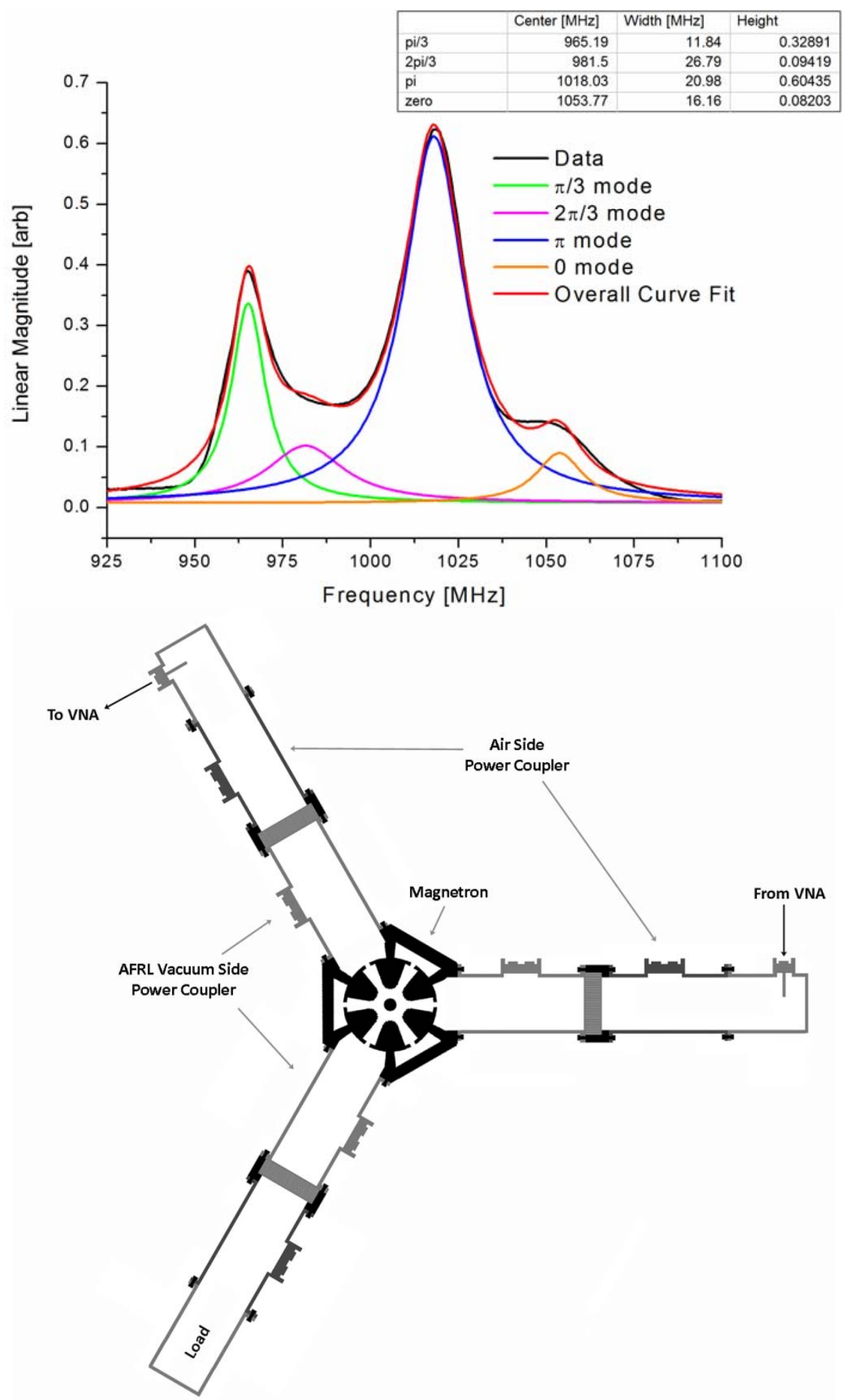

UM/L-3 mode spectrum. S21 measurement performed using vector network analyzer (VNA). 


\section{Appendix E \\ ICEPIC Cold Test Data}

\begin{tabular}{|c|c|c|c|c|}
\hline \multicolumn{5}{|l|}{ Excited mode: } \\
\hline $\begin{array}{l}\stackrel{\mathrm{v}}{\text { Standard Cathode }} \\
\text { Measured: }\end{array}$ & 0 & $\mathrm{PI} / 3$ & $2 \mathrm{PI} / 3$ & PI \\
\hline 0 & $\begin{array}{l}\text { 1. } 0275 \mathrm{GHz} \\
+-2 \mathrm{MHz}\end{array}$ & & & \\
\hline $\mathrm{PI} / 3$ & & $\begin{array}{l}961.6 \mathrm{MHz} \\
+-2 \mathrm{MHz}\end{array}$ & $961.6 \mathrm{MHz}$ & \\
\hline $2 \mathrm{PI} / 3$ & & $1.027 \mathrm{GHz}$ & $\begin{array}{l}1.027 \mathrm{GHz} \\
+-.002 \mathrm{GHz}\end{array}$ & \\
\hline PI & & & $\begin{array}{l}1.026 \mathrm{GHZ} \\
+-.002 \mathrm{GHz}\end{array}$ & $\begin{array}{l}1.030 \mathrm{GHz} \\
+-.003 \mathrm{GHz}\end{array}$ \\
\hline
\end{tabular}




\section{Appendix F}

Simulated 6 Vane Magnetron Mode Spectrum

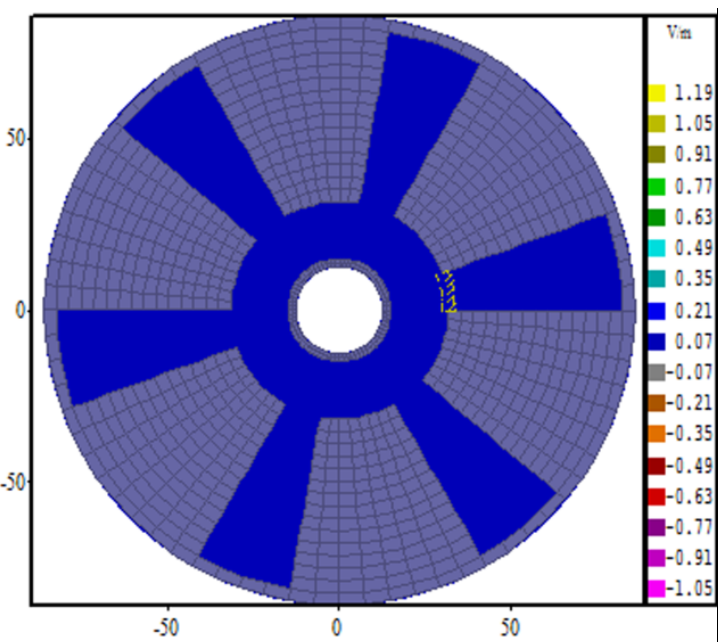

$\mathbf{0}_{0}$ frequency $=0 \mathrm{MHz}$

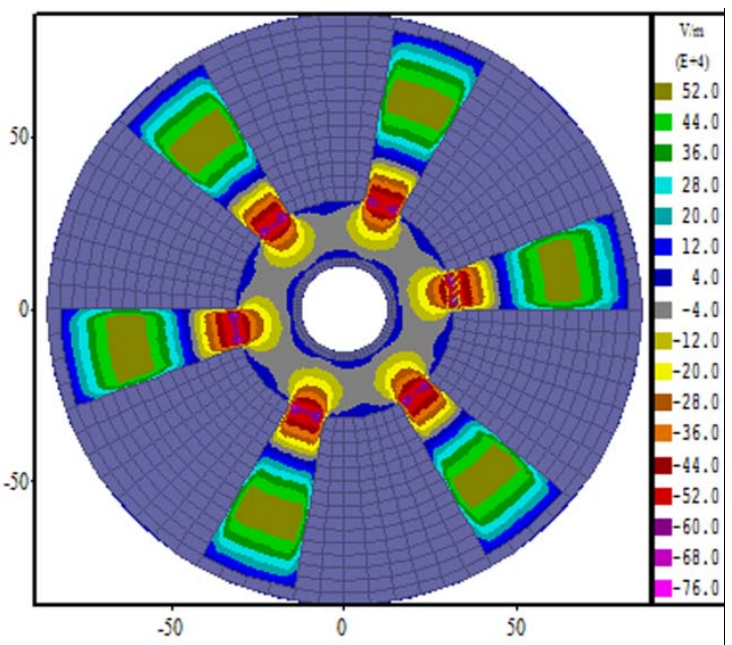

$\mathbf{0}_{2}$

frequency $=4163 \mathrm{MHz}$

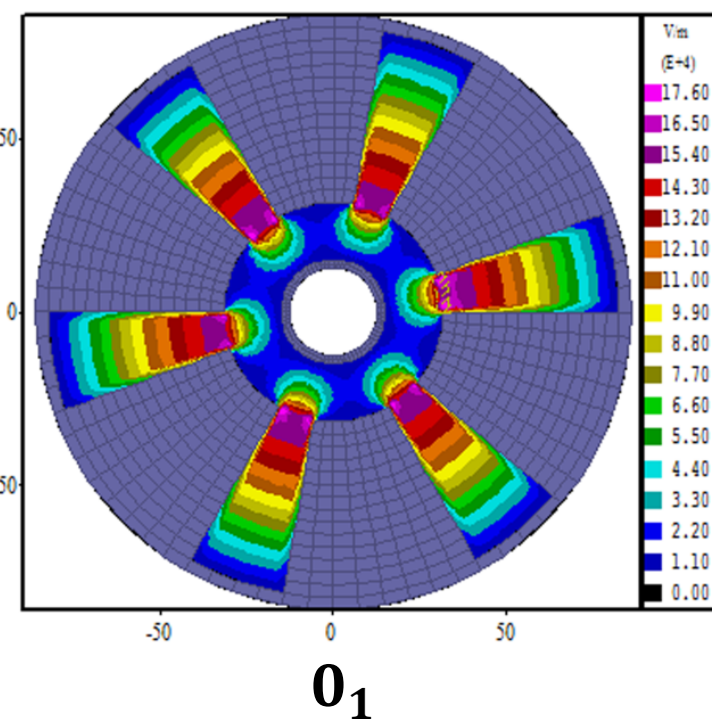

frequency $=1837 \mathrm{MHz}$

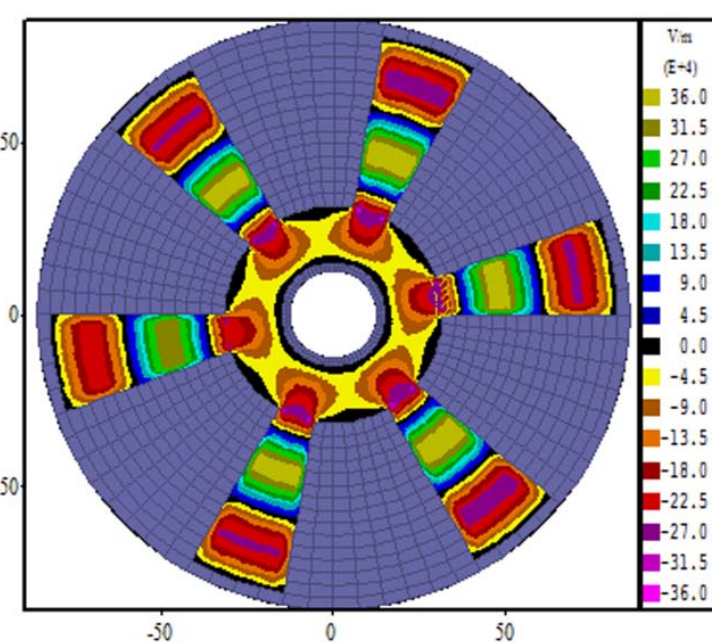

$\mathbf{0}_{3}$

frequency $=6648 \mathrm{MHz}$

The first four radial variations for the $0(2 \pi)$ mode of a cold (no beam) 6 vane simulation magnetron (this is not the same simulated magnetron used for the magnetic priming research). Only the $E_{\theta}$ field component is plotted.

Distance scales are in $\mathbf{~ m m}$. 


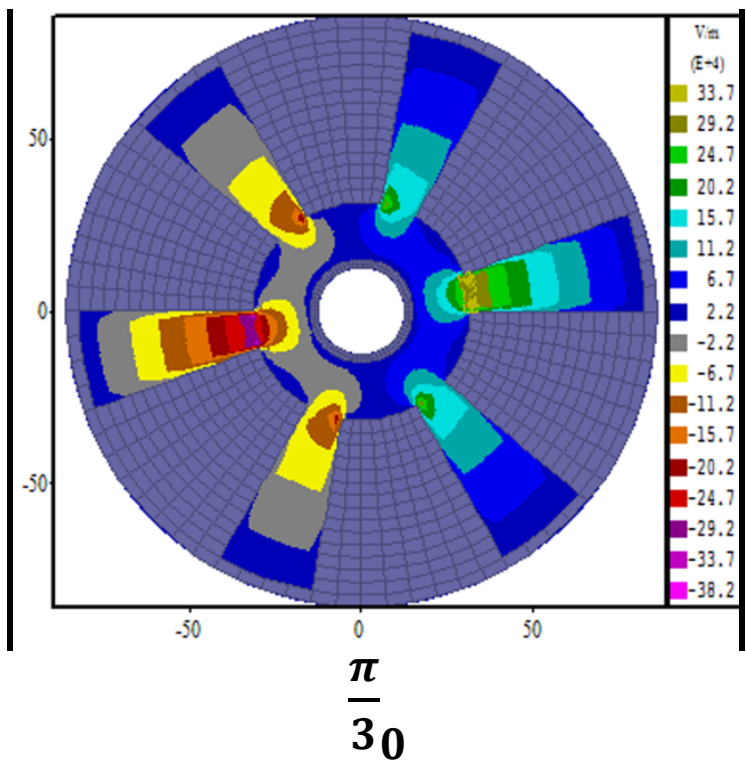

frequency $=808 \mathrm{MHz}$
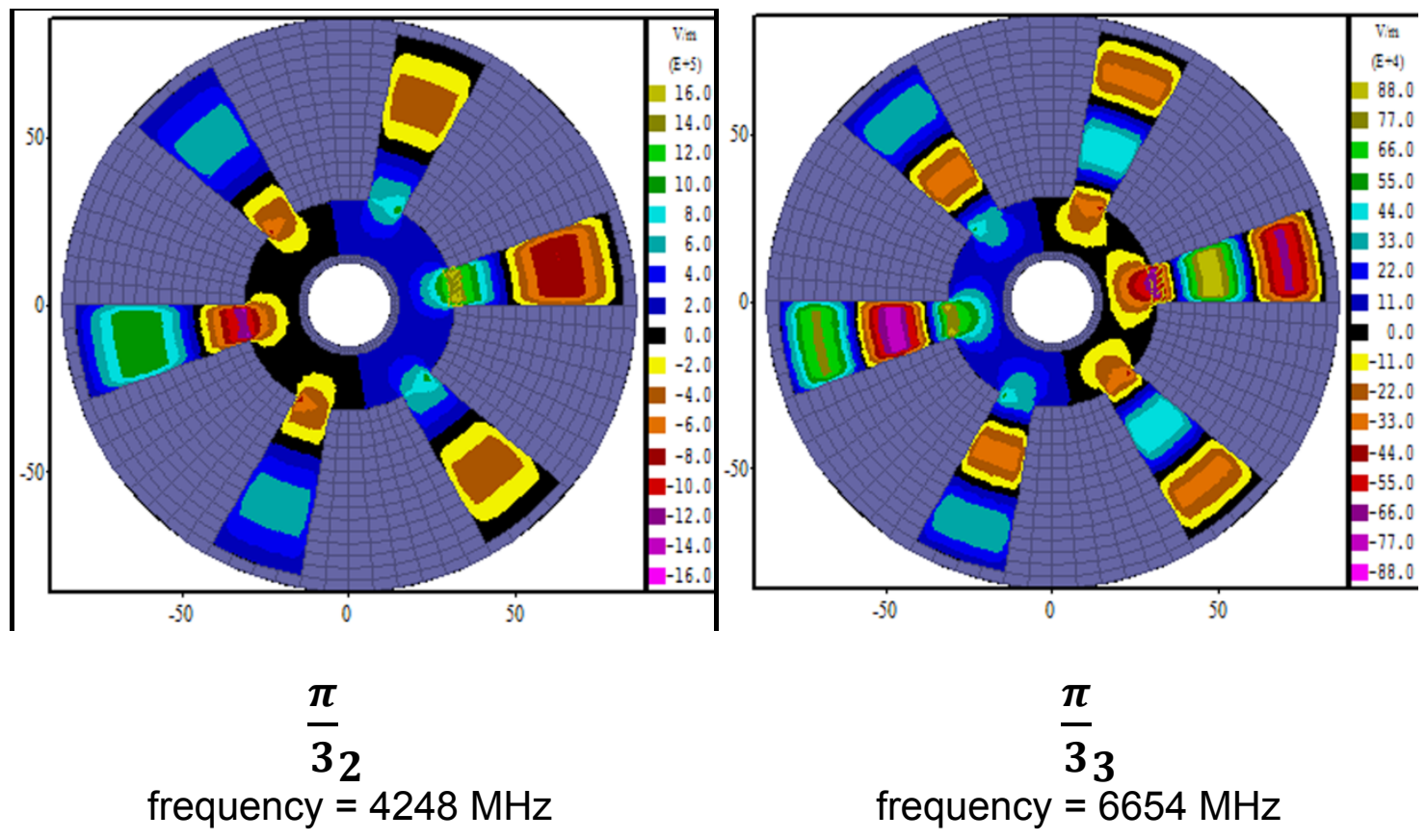

The first four radial variations for the $\pi / 3$-mode of a cold (no beam) 6 vane simulation magnetron (this is not the same simulated magnetron used for the magnetic priming research). Only the $E_{\theta}$ field component is plotted. Distance scales are in $\mathrm{mm}$. 


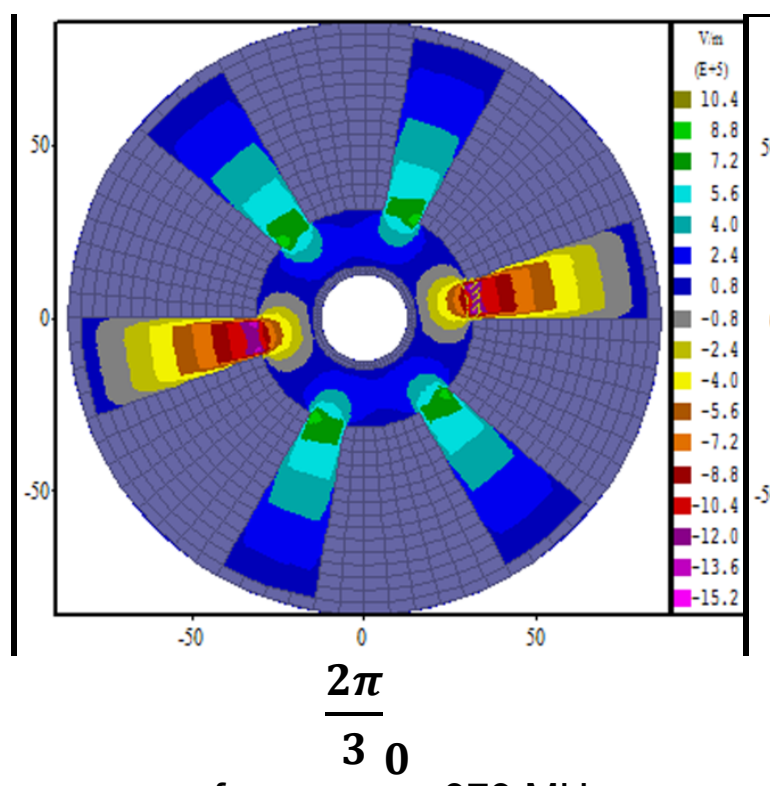

frequency $=979 \mathrm{MHz}$

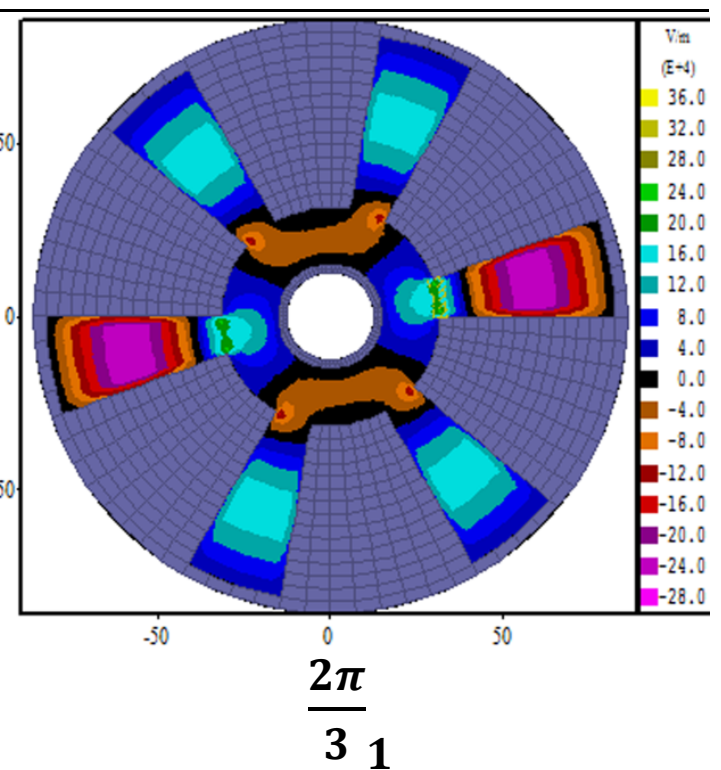

frequency $=3498 \mathrm{MHz}$
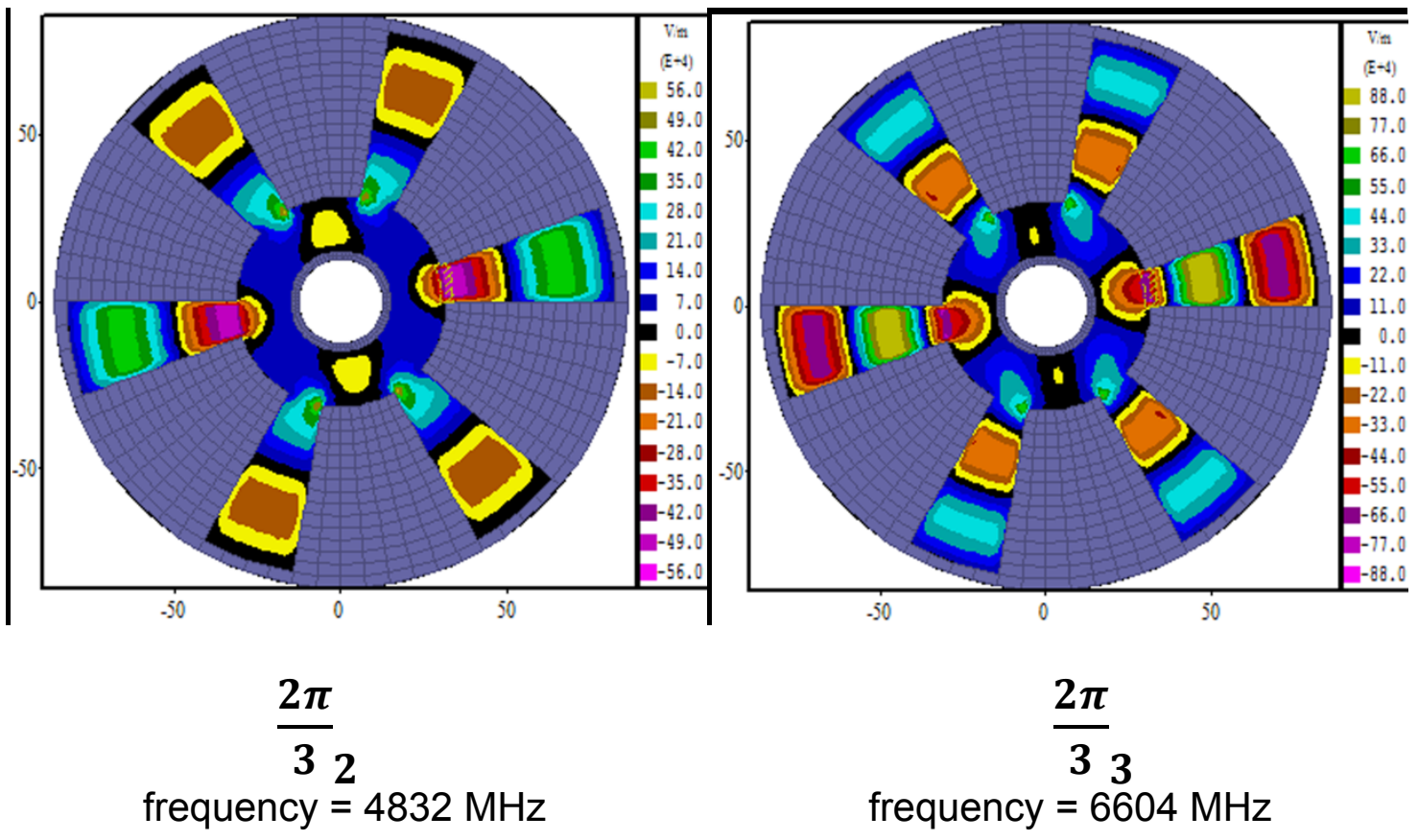

The first four radial variations for the $2 \pi / 3$-mode of a cold (no beam) 6 vane simulation magnetron (this is not the same simulated magnetron used for the magnetic priming research). Only the $E_{\theta}$ field component is plotted. Distance scales are in $\mathrm{mm}$. 


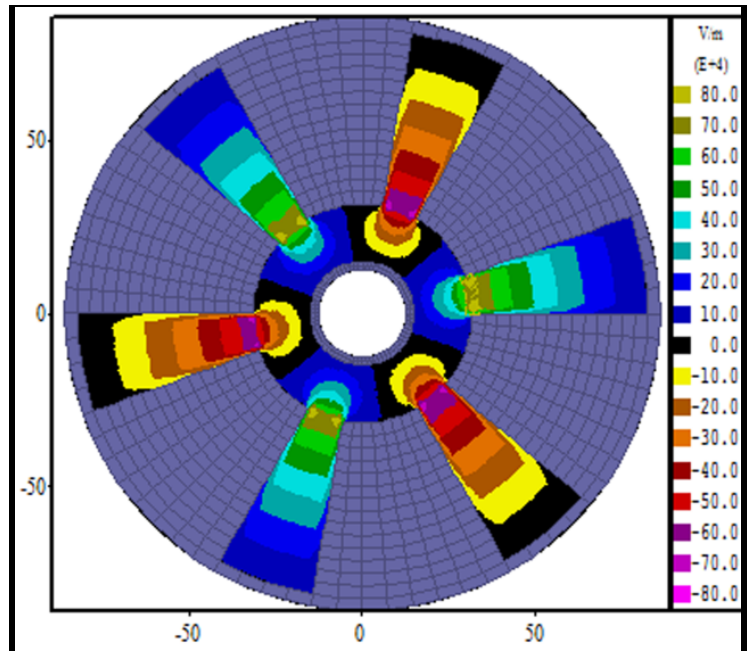

$\boldsymbol{\pi}_{\mathbf{0}}$

frequency $=1006 \mathrm{MHz}$

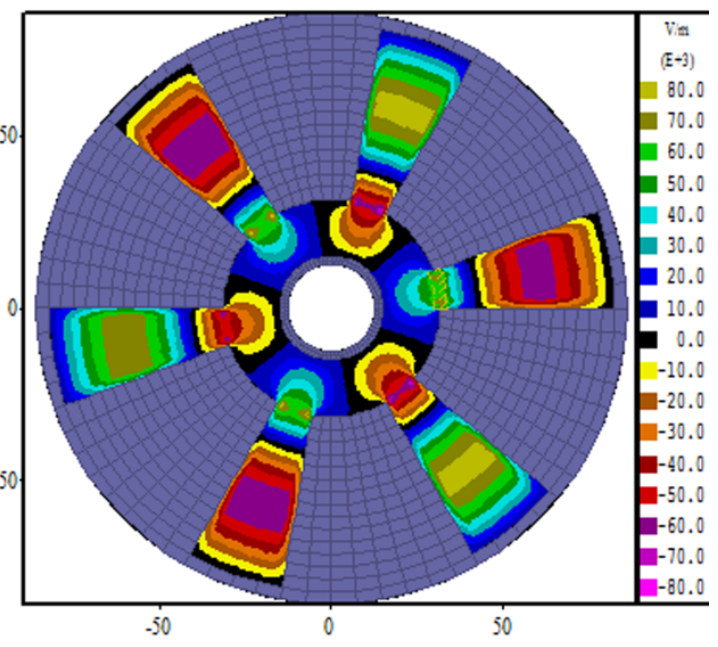

$\pi_{1}$

frequency $=3714 \mathrm{MHz}$

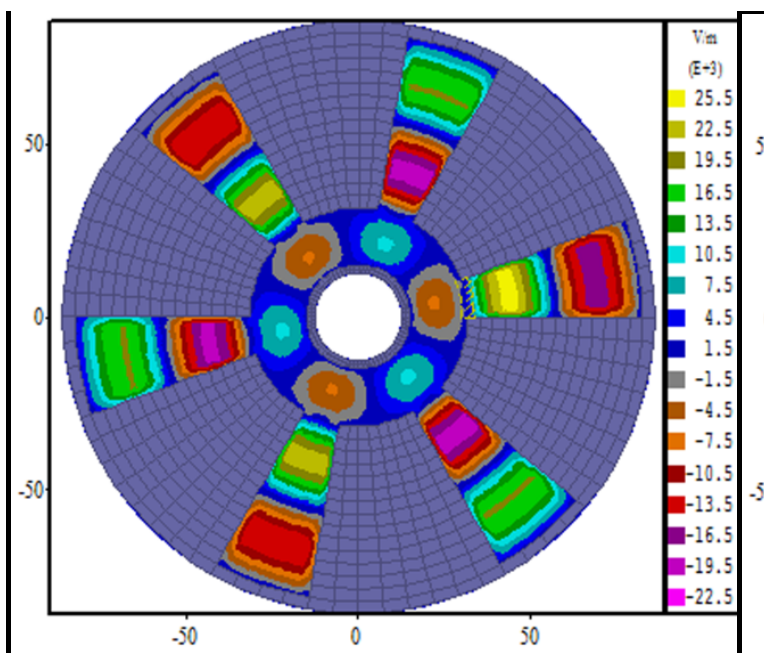

$\pi_{2}$

frequency $=5939 \mathrm{MHz}$

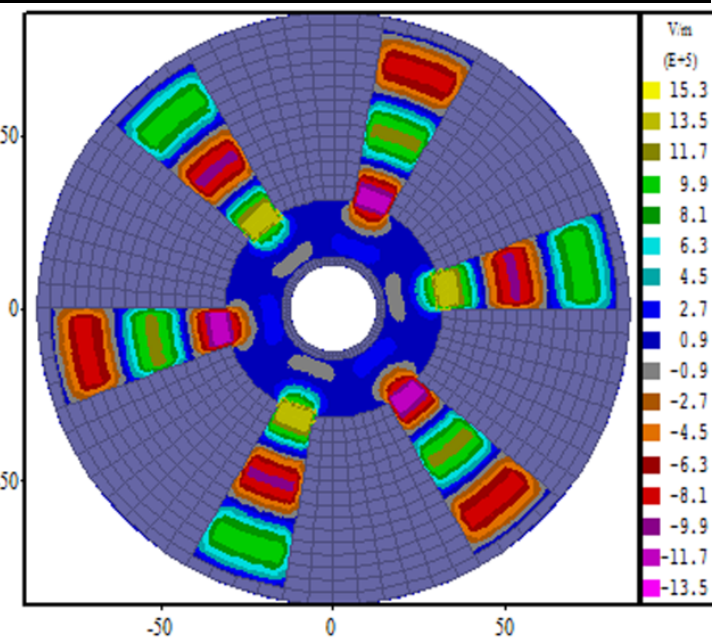

$\boldsymbol{\pi}_{\mathbf{3}}$

frequency $=7614 \mathrm{MHz}$

The first four radial variations for the $\pi$-mode of a cold (no beam) 6 vane simulation magnetron (this is not the same simulated magnetron used for the magnetic priming research). Only the $E_{\theta}$ field component is plotted.

Distance scales are in $\mathrm{mm}$. 


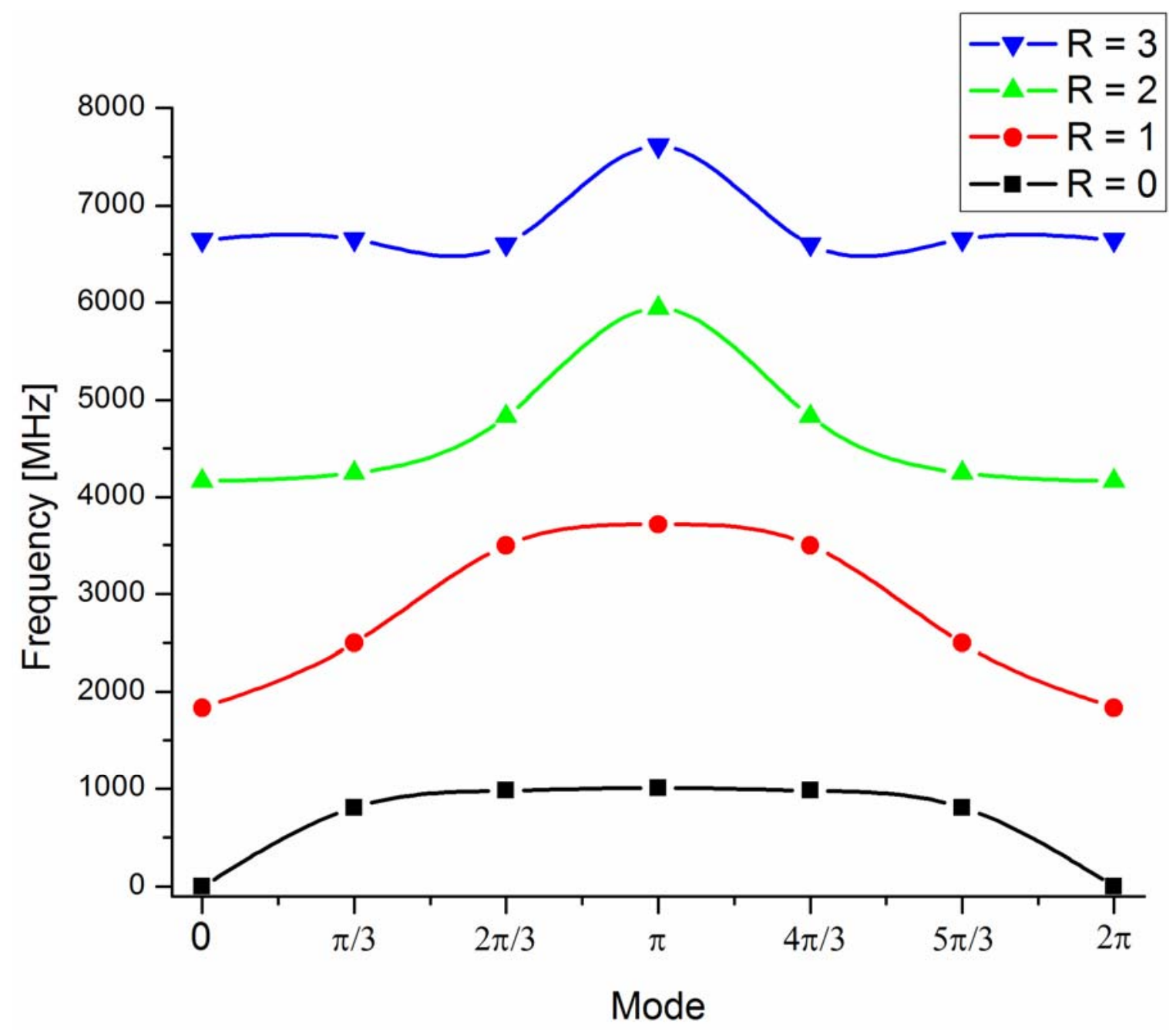

Brillouin dispersion plot for the simulated 6 vane magnetron depicted on pages 153-156 


\section{Bibliography}




\section{Bibliography}

[1] R.M. Gilgenbach, Y. Lau, H. McDowell, K.L. Cartwright, and T.A. Spencer, "Crossed-Field Devices," Modern Microwave and Millimeter Wave Power Electronics, R.J. Barker, N.C. Luhmann, J.H. Booske, and G.S. Nusinovich, eds., Piscataway, NJ: Wiley-IEEE Press, 2005.

[2] Y.Y. Lau, "Theory of Crossed-Field Devices and a Comparative Study of Other Radiation Sources," High Power Microwave Sources, V.L. Granatstein and I. Alexeff, eds., Norwood, MA: Artech House, 1987.

[3] A.S. Gilmour, Jr., Microwave Tubes, Norwood, MA: Artech House, 1986.

[4] G.B. Collins, ed., Microwave Magnetrons, New York: McGraw-Hill, 1948.

[5] F.J. Agee, W.L. Baker, and J.A. Gaudet, "HPM Sources: The DoD Perspective," High-Power Microwave Sources and Technologies, R.J. Barker and E. Schamiloglu, eds., Wiley-IEEE Press, 2001.

[6] T.A. Spencer, "Current HPM Source Research," HIGH ENERGY DENSITY AND HIGH POWER RF: 6th Workshop on High Energy Density and High Power RF, Berkeley Springs, West Virginia (USA): AIP, 2003, p. 46.

[7] J. Benford, J.A. Swegle, and E. Schamiloglu, High Power Microwaves, London, U.K.: Taylor \& Francis, 2007.

[8] J. Benford, "Relativistic Magnetrons," High Power Microwave Sources, V.L. Granatstein and I. Alexeff, eds., Norwood, MA: Artech House, 2007.

[9] R.W. Lemke, T.C. Genoni, and T.A. Spencer, "Three-dimensional particle-incell simulation study of a relativistic magnetron," Physics of Plasmas, vol. 6, Feb. 1999, pp. 603-613.

[10] V.B. Neculaes, R.M. Gilgenbach, and Y.Y. Lau, "Low-noise microwave magnetrons by azimuthally varying axial magnetic field," Applied Physics Letters, vol. 83, 2003, pp. 1938-1940.

[11] V. Neculaes, "Magnetron Magnetic Priming for Rapid Startup and Noise Reduction," Dissertation (Ph.D.), University of Michigan, 2005.

[12] V. Neculaes, M. Jones, R. Gilgenbach, Y. Lau, J. Luginsland, B. Hoff, W. White, N. Jordan, P. Pengvanich, Y. Hidaka, and H. Bosman, "Magnetic priming effects on noise, startup, and mode competition in magnetrons," Plasma Science, IEEE Transactions on, vol. 33, 2005, pp. 94-102. 
[13] V. Neculaes, M. Jones, R. Gilgenbach, Y. Lau, J. Luginsland, B. Hoff, W. White, N. Jordan, P. Pengvanich, Y. Hidaka, and H. Bosman, "Magnetic perturbation effects on noise and startup in DC-operating oven magnetrons," IEEE Trans. Electron Devices, vol. 52, 2005, pp. 864-871.

[14] V. Neculaes, R.M. Gilgenbach, and Y.Y. Lau, "Low noise crossed field devices such as a microwave magnetron having and azimuthally varying axial magnetic field and microwave oven utilizing same," U.S. Patent 6872 929.

[15] V. Neculaes, R.M. Gilgenbach, and Y.Y. Lau, "Low noise crossed field devices sucha as a microwave magnetron having an azimuthally varying axial magnetic field and microwave oven utilizing same," U.S. Patent 6921 890.

[16] M. Jones, V. Neculaes, W. White, Y. Lau, R. Gilgenbach, J. Luginsland, P. Pengvanich, N. Jordan, Y. Hidaka, and H. Bosman, "Simulations of magnetic priming in a relativistic magnetron," Electron Devices, IEEE Transactions on, vol. 52, 2005, pp. 858-863.

[17] M. Jones, V. Neculaes, W. White, Y. Lau, R. Gilgenbach, J. Luginsland, P. Pengvanich, N. Jordan, Y. Hidaka, and H. Bosman, "Simulation of rapid startup in microwave magnetrons with azimuthally-varying axial magnetic fields," Fifth IEEE International Vacuum Electronics Conference, 2004., 2004, pp. 168-169.

[18] M.C. Jones, V.B. Neculaes, W. White, Y.Y. Lau, and R.M. Gilgenbach, "Simulation of rapid startup in microwave magnetrons with azimuthally varying axial magnetic fields," Applied Physics Letters, vol. 84, Feb. 2004, pp. 1016-1018.

[19] V. Neculaes, R. Gilgenbach, Yue Ying Lau, M. Jones, and W. White, "Lownoise microwave oven magnetrons with fast start-oscillation by azimuthally varying axial magnetic fields," IEEE Trans Plasma Sci., vol. 32, 2004, pp. 1152-1159.

[20] M.C. Jones, V.B. Neculaes, Y.Y. Lau, R.M. Gilgenbach, W.M. White, B.W. Hoff, and N.M. Jordan, "Magnetron priming by multiple cathodes," Applied Physics Letters, vol. 87, 2005, pp. 081501-3.

[21] J.W. Luginsland, Y.Y. Lau, V.B. Neculaes, R.M. Gilgenbach, M.C. Jones, M.H. Frese, and J.J. Watrous, "Three-dimensional particle-in-cell simulations of rapid start-up in strapped oven magnetrons due to variation in the insulating magnetic field," Applied Physics Letters, vol. 84, Jun. 2004, pp. 5425-5427. 
[22] M. Jones, "Cathode priming of a relativistic magnetron using multi-emission zones on projection ablation lithography cathodes.," Dissertation (Ph.D.), University of Michigan, 2005.

[23] M. Jones, R. Gilgenbach, W. White, M. Lopez, V. Neculaes, Y. Lau, T. Spencer, and D. Price, "Projection ablation lithography cathodes for a high current relativistic magnetron," The 31st IEEE International Conference on Plasma Science, 2004., 2004, p. 276.

[24] R. Gilgenbach, M. Jones, V. Neculaes, Y. Lau, W. White, N. Jordan, B. Hoff, R. Edgar, P. Pengvanich, and Y. Hidaka, "Cathode priming of magnetrons for rapid startup and mode-locking," The Joint 30th International Conference on Infrared and Millimeter Waves and 13th International Conference on Terahertz Electronics, 2005. (IRMMW-THz 2005)., 2005, pp. 535-536 vol. 2.

[25] M.C. Jones, V.B. Neculaes, R.M. Gilgenbach, W.M. White, M.R. Lopez, Y.Y. Lau, T.A. Spencer, and D. Price, "Projection ablation lithography cathode for high-current, relativistic magnetron," Review of Scientific Instruments, vol. 75, 2004, pp. 2976-2980.

[26] S. Prasad, H. Bosman, M. Fuks, and E. Schamiloglu, "Efficiency enhancement in AG magnetron with transparent cathode," The 33rd IEEE International Conference on Plasma Science, 2006. (ICOPS 2006), 2006, p. 298.

[27] M. Fuks and E. Schamiloglu, "Rapid Start of Oscillations in a Magnetron with a "Transparent" Cathode," Physical Review Letters, vol. 95, Nov. 2005, p. 205101.

[28] J. Fleming, P. Mardahl, L. Bowers, H. Bosman, S. Prasad, M. Fuks, and E. Schamiloglu, "Three dimensional PIC simulations of the transparent and eggbeater cathodes in the Michigan relativistic," Plasma Science, 2006. ICOPS 2006. IEEE Conference Record - Abstracts. The 33rd IEEE International Conference on, 2006, p. 338.

[29] J.I. Kim, J.H. Won, and G.S. Park, "Electron prebunching in microwave magnetron by electric priming using anode shape modification," Applied Physics Letters, vol. 86, Apr. 2005, pp. 171501-3.

[30] J.I. Kim, J.H. Won, G.S. Park, H.J. Ha, and J.C. Shon, "Reduction of noise in strapped magnetron by electric priming using anode shape modification," Applied Physics Letters, vol. 88, May. 2006, pp. 221501-3.

[31] W.M. White, "RF priming of a long pulse relativistic magnetron," Dissertation (Ph.D.), University of Michigan, 2006. 
[32] P. Pengvanich, Y.Y. Lau, J.W. Luginsland, R.M. Gilgenbach, E. Cruz, and E. Schamiloglu, "Effects of frequency chirp on magnetron injection locking," Physics of Plasmas, vol. 15, Jul. 2008, pp. 073110-6.

[33] P. Pengvanich, V.B. Neculaes, Y.Y. Lau, R.M. Gilgenbach, M.C. Jones, W.M. White, and R.D. Kowalczyk, "Modeling and experimental studies of magnetron injection locking," Journal of Applied Physics, vol. 98, Dec. 2005, pp. 114903-6.

[34] P. Pengvanich, V. Neculaes, Y. Lau, R. Gilgenbach, M. Jones, W. White, and R. Kowalczyk, "Modeling and Experimental Studies of Magnetron Injection Locking," IEEE International Conference on Plasma Science, 2005., 2005, p. 211.

[35] P. Pengvanich, Y.Y. Lau, E. Cruz, R.M. Gilgenbach, B. Hoff, and J.W. Luginsland, "Analysis of peer-to-peer locking of magnetrons," Physics of Plasmas, vol. 15, Oct. 2008, pp. 103104-4.

[36] B. Hoff, R. Gilgenbach, N. Jordan, Y. Lau, E. Cruz, D. French, M. Gomez, J. Zier, T. Spencer, and D. Price, "Magnetic Priming at the Cathode of a Relativistic Magnetron," Plasma Science, IEEE Transactions on, vol. 36, 2008, pp. 710-717.

[37] B.W. Hoff, P.J. Mardahl, R.M. Gilgenbach, M.D. Haworth, D.M. French, Y.Y. Lau, and M. Franzi, "Microwave Window Breakdown Experiments and Simulations on the UM/L-3 Relativistic Magnetron," Review of Scientific Instruments, Accepted for Publication. .

[38] J.C. Slater, Microwave Electronics, New York: D. Van Nostrand Company, Inc., 1954.

[39] D.J. Kaup and G. Thomas, "Density profile and current flow in a crossedfield amplifier," Journal of Plasma Physics, vol. 58, 1997, pp. 145-161.

[40] F.F. Chen, Introduction to Plasma Physics and Controlled Fusion, New York: Plenum Press, 1984.

[41] L. Brillouin, "A Theorem of Larmor and Its Importance for Electrons in Magnetic Fields," Physical Review, vol. 67, Apr. 1945, p. 260.

[42] R.V. Lovelace and E. Ott, "Theory of magnetic insulation," Physics of Fluids, vol. 17, Jun. 1974, pp. 1263-1268.

[43] A.L. Garner, Y.Y. Lau, and D. Chernin, "Collapse of cycloidal electron flows induced by misalignments in a magnetically insulated diode," Physics of Plasmas, vol. 5, Jun. 1998, pp. 2447-2453. 
[44] P.J. Christenson, D.P. Chernin, A.L. Garner, and Y.Y. Lau, "Resistive destabilization of cycloidal electron flow and universality of (near-) Brillouin flow in a crossed-field gap," Physics of Plasmas, vol. 3, Dec. 1996, pp. 4455-4462.

[45] P.J. Christenson, "Equilibrium, Stability, and Turbulence in Cycloidal Electron Flows in Crossed Electric and Magnetic Fields," Ph.D. Dissertation, University of Michigan, 1996.

[46] P.J. Christenson and Y.Y. Lau, "One-Dimensional Modulational Instability in a Crossed-Field Gap," Physical Review Letters, vol. 76, Apr. 1996, p. 3324.

[47] R. Meredith, Engineers' Handbook of Industrial Microwave Heating, London, U.K.: The institution of Electrical Engineers, 1998.

[48] R. Lemke, T. Genoni, and T. Spencer, "Effects that limit efficiency in relativistic magnetrons," Plasma Science, IEEE Transactions on, vol. 28, 2000, pp. 887-897.

[49] V. Neculaes, P. Pengvanich, Y. Hidaka, Y. Lau, R. Gilgenbach, W. White, M. Jones, H. Bosman, and J. Luginsland, "Rapid kinematic bunching and parametric instability in a crossed-field gap with a periodic magnetic field," Plasma Science, IEEE Transactions on, vol. 33, 2005, pp. 654-660.

[50] P. Pengvanich, "Theory of Injection Locking and Rapid Start-Up of Magnetrons, and Effects of Manufacturing Errors in Terahertz Traveling Wave Tubes.," Dissertation (Ph.D.), University of Michigan, 2007.

[51] S. Humphries, Advanced Magnetic Field Design Suite, Including Magnum and MetaMesh, Albuquerque, NM: Field Precisions, LLC., .

[52] W.T. McLyman, Transformer and inductor design handbook, Boca Raton, FL: CRC Press, Taylor and Francis Group, 2004.

[53] M. Lopez, R. Gilgenbach, M. Jones, W. White, D. Jordan, M. Johnston, T. Strickler, V. Neculaes, Yue Ying Lau, T. Spencer, M. Haworth, K. Cartwright, P. Mardahl, J. Luginsland, and D. Price, "Relativistic magnetron driven by a microsecond E-beam accelerator with a ceramic insulator," Plasma Science, IEEE Transactions on, vol. 32, 2004, pp. 1171-1180.

[54] M.R.L. Lopez, "Experiments on a Relativistic Magnetron Driven by a Microsecond Electron Beam Accelerator With a Ceramic Insulating Stack," Dissertation (Ph.D.), University of Michigan, 2003.

[55] H. Sze, B. Harteneck, J. Benford, and T.S.T. Young, "Operating Characteristics of a Relativistic Magnetron with a Washer Cathode," IEEE Trans Plasma Sci., vol. 15, 1987, pp. 327-334. 
[56] W.J. Williams, Time Frequency Toolbox, Ann Arbor, MI: Quantum Signal, LLC., .

[57] B. Hoff, R. Gilgenbach, N. Jordan, Y. Lau, E. Cruz, D. French, M. Gomez, J. Zier, T. Spencer, and D. Price, "Magnetic Priming at the Cathode of a Relativistic Magnetron," Plasma Science, IEEE Transactions on, vol. 36, 2008, pp. 710-717.

[58] E. Ott and R.V. Lovelace, "Magnetic insulation and microwave generation," Applied Physics Letters, vol. 27, Oct. 1975, pp. 378-380.

[59] R.V. Lovelace and T.F.T. Young, "Relativistic Hartree condition for magnetrons: Theory and comparison with experiments," Physics of Fluids, vol. 28, 1985, pp. 2450-2452.

[60] D. Price and J. Benford, "General scaling of pulse shortening in explosiveemission-driven microwave sources," Plasma Science, IEEE Transactions on, vol. 26, 1998, pp. 256-262.

[61] D. Price, J. Levine, and J. Benford, "Diode plasma effects on the microwave pulse length from relativistic magnetrons," Plasma Science, IEEE Transactions on, vol. 26, 1998, pp. 348-353.

[62] Y.Y. Lau, J.W. Luginsland, K.L. Cartwright, and M.D. Haworth, "Role of lons in a Crossed-Field Diode," Physical Review Letters, vol. 98, Jan. 2007, pp. 015002-4.

[63] P. Mardahl, K. Cartwright, A. Greenwood, M. Haworth, L. Bowers, T. Murphy, T. Spencer, R. Gilgenbach, M. Lopez, J. Watrous, and J. Luginsland, "Numerical model of the MELBA-C relativistic magnetron using 3D PIC," American Physical Society, 45th Annual Meeting of the Division of Plasma Physics, Albuquerque, NM: American Physical Society, 2003.

[64] P. Mardahl, "Energy losses in simulated relativistic Michigan A6 magnetron with shaped cathode," IVEC 2008. IEEE International Vacuum Electronics Conference, 2008, 2008, p. 265.

[65] C. Fichtl, K. Cartwright, P. Mardahl, and J. Verboncoeur, "Self-consistent simulation of multipactor discharge at HPM dielectric windows," The 31st IEEE International Conference on Plasma Science, 2004., 2004, p. 126.

[66] K. Cartwright, P. Mardahl, M. Haworth, V. Neculaes, M. Jones, M. Lopez, Y. Lau, and R. Gilgenbach, "Simulation of a relativistic magnetron with a varying axial magnetic field," The 31st IEEE International Conference on Plasma Science, 2004, 2004, p. 275.

[67] T. Fleming, P. Mardahl, L. Bowers, and K. Cartwright, "Three Dimensional PIC Simulations of Novel Cathodes in the Michigan and AFRL Relativistic 
Magnetrons," Conference Record of the Twenty-Seventh International Power Modulator Symposium, 2006, 2006, pp. 401-404.

[68] A. Valfells, L.K. Ang, Y.Y. Lau, and R.M. Gilgenbach, "Effects of an external magnetic field, and of oblique radio-frequency electric fields on multipactor discharge on a dielectric," Physics of Plasmas, vol. 7, Feb. 2000, pp. 750757.

[69] R.A. Kishek and Y.Y. Lau, "Multipactor Discharge on a Dielectric," Physical Review Letters, vol. 80, Jan. 1998, p. 193.

[70] N.M. Jordan, Y.Y. Lau, D.M. French, R.M. Gilgenbach, and P. Pengvanich, "Electric field and electron orbits near a triple point," Journal of Applied Physics, vol. 102, 2007, pp. 033301-10.

[71] Y.Y. Lau, J.P. Verboncoeur, and H.C. Kim, "Scaling laws for dielectric window breakdown in vacuum and collisional regimes," Applied Physics Letters, vol. 89, Dec. 2006, pp. 261501-3.

[72] H. Kim, J. Verboncoeur, and Y. Lau, "Power Modulators and Repetitive Pulsed Power," Dielectrics and Electrical Insulation, IEEE Transactions on, vol. 14, 2007, pp. 766-773.

[73] G. Blaise and C.L. Gressus, "Charge Trapping-Detrapping Processes and Related Breakdown Phenomena," High Voltage Vacuum Insulation: Basic Concepts and Technological Practice, R. Latham, ed., San Diego, CA: Academic Press, 1995, pp. 330-401.

[74] R. Kishek, "Interaction of multipactor discharge and rf structures," Dissertation (Ph.D.), University of Michigan, 1997.

[75] J. Verboncoeur, H. Kim, Y. Chen, and Y. Lau, "Modeling RF Window Breakdown: From Vacuum Multipactor to Volumetric lonization Discharge," IEEE Transactions on Dielectrics and Electrical Insulation, vol. 14, Aug. 2007, pp. 766-773.

[76] A. Valfells, J. Verboncoeur, and Y. Lau, "Space-charge effects on multipactor on a dielectric," Plasma Science, IEEE Transactions on, vol. 28, 2000, pp. 529-536.

[77] W. Kalbreier and B. Goddard, "Radiation-triggered breakdown phenomena in high-energy e+e- colliders," Electrical Insulation, IEEE Transactions on, vol. 28, 1993, pp. 444-453.

[78] J. Krile, A. Neuber, and H. Krompholz, "Effects of UV Illumination on Surface Flashover Under Pulsed Excitation," Plasma Science, IEEE Transactions on, vol. 36, 2008, pp. 332-340. 
[79] G. Edmiston, A. Neuber, L. McQuage, J. Krile, H. Krompholz, and J. Dickens, "Contributing Factors to Window Flashover under Pulsed High Power Microwave Excitation at High Altitude," Dielectrics and Electrical Insulation, IEEE Transactions on, vol. 14, 2007, pp. 783-789.

[80] K. Hendricks and M. Haworth, "Experiments on high-power microwave transmission through a belljar," Plasma Science, IEEE Transactions on, vol. 30, 2002, pp. 1215-1219.

[81] R.B. Anderson, W.D. Getty, M.L. Brake, Y.Y. Lau, R.M. Gilgenbach, and A. Valfells, "Multipactor experiment on a dielectric surface," Review of Scientific Instruments, vol. 72, Jul. 2001, pp. 3095-3099.

[82] D. Hemmert, A. Neuber, J. Dickens, H. Krompholz, L. Hatfield, and M. Kristiansen, "Microwave magnetic field effects on high-power microwave window breakdown," Plasma Science, IEEE Transactions on, vol. 28, 2000, pp. 472-477.

[83] David John Hemmert, "Window and cavity breakdown caused by high power microwaves," Thesis (M.S.), Texas Tech University, 1998.

[84] A. Neuber, J. Dickens, D. Hemmert, H. Krompholz, L. Hatfield, and M. Kristiansen, "Window and cavity breakdown caused by high power microwaves," 11th IEEE International Pulsed Power Conference, 1997., 1997, pp. 135-140 vol.1.

[85] T. Treado, R. Smith, C. Shaughnessy, and G. Thomas, "Temporal study of long-pulse relativistic magnetron operation," Plasma Science, IEEE Transactions on, vol. 18, 1990, pp. 594-602.

[86] T. Fleming, P. Mardahl, L. Bowers, K. Cartwright, M. Bettencourt, and M. Haworth, "Virtual Prototyping of Novel Cathode Designs for the Relativistic Magnetron," Computing in Science \& Engineering, vol. 9, 2007, pp. 18-28.

[87] R. Miller, Y.Y. Lau, and J.H. Booske, "Electric field distribution on knife-edge field emitters," Applied Physics Letters, vol. 91, 2007, pp. 074105-3.

[88] R. Miller, Y. Lau, and J. Booske, "Effective Current Enhancement vs. Aspect Ratio for Rectangular Ridge Cathodes," IEEE 34th International Conference on Plasma Science, 2007, 2007, p. 136.

[89] R. Miller, Yue Yin Lau, and J. Booske, "Field enhancement on knife-edge cathodes," IEEE International Vacuum Electronics Conference, 2008, 2008 , p. 353.

[90] H.C. Miller, "High Voltage Performance Characteristics of Solid Insulators in Vacuum," High Voltage Vacuum Insulation: Basic Concepts and 
Technological Practice, R. Latham, ed., San Diego, CA: Academic Press, 1995, pp. 299-328.

[91] Science Research Corporation, Studies on temporal field emission from stainless steel surfaces: Final report, April 30, 1987 to June 30, 1987, United States: National Technical Information Service, 1987.

[92] D.R. Lide, CRC Handbook of Chemistry and Physics, 86th Edition Edited, Boca Raton, FL: CRC Press, Taylor and Francis Group, 2005.

[93] J. Vaughan, "A new formula for secondary emission yield," Electron Devices, IEEE Transactions on, vol. 36, 1989, pp. 1963-1967.

[94] R. Vaughan, "Secondary emission formulas," Electron Devices, IEEE Transactions on, vol. 40, 1993, p. 830.

[95] M. Boubaya, "Charging regime of PMMA studied by secondary electron emission," The European Physical Journal Applied Physics, vol. 37, 2007, p. 79.

[96] O. Hachenberg and W. Brauer, "Secondary Electron Emission from Solids," Advances in Electronics and Electron Physics, L. Marton, ed., New York: Academic Press, 1959.

[97] A. Shih and C. Hor, "Secondary emission properties as a function of the electron incidence angle," Electron Devices, IEEE Transactions on, vol. 40, 1993, pp. 824-829.

[98] H. Kitabayashi, K. Tsuji, and K. Itoh, "A streaming electrification model based on differences of work function between solid materials and insulating oil," Journal of Electrostatics, vol. 63, Jun. 2005, pp. 735-741.

[99] Y.Y. Lau, "Analysis of Multipactor Discharge. Final Technical Report, April 2, 1998 - August 31, 2005," Performer: Michigan Univ., DC. . 26p. 2005.

[100] C. Chang, G. Liu, C. Tang, C. Chen, S. Qiu, J. Fang, and Q. Hou, "The influence of desorption gas to high power microwave window multipactor," Physics of Plasmas, vol. 15, 2008, pp. 093508-6.

[101] A. Neuber, M. Butcher, H. Krompholz, L. Hatfield, and M. Kristiansen, "The role of outgassing in surface flashover under vacuum," Plasma Science, IEEE Transactions on, vol. 28, 2000, pp. 1593-1598. 